\title{
Stable 3,6-Linked Fluorenyl Radical Oligomers with Intramolecular Anti- ferromagnetic Coupling and Polyradical Characters
}

Xuefeng Lu, ${ }^{\dagger}$ Sangsu Lee, ${ }^{*}$ Jun Oh Kim, ${ }^{*}$ Tullimilli Y. Gopalakrishna, ${ }^{\dagger}$ Hoa Phan, ${ }^{\dagger}$ Tun Seng Herng, ${ }^{\S}$ Zhenglong Lim, ${ }^{\dagger}$ Zebing Zeng," Jun Ding, ${ }^{\S}$ Dongho Kim ${ }^{*}$ and Jishan Wu, ${ }^{*}{ }^{\dagger}$

${ }^{\dagger}$ Department of Chemistry, National University of Singapore, 3 Science Drive 3, 117543, Singapore

${ }^{*}$ Spectroscopy Laboratory for Functional $\pi$-Electronic Systems and Department of Chemistry, Yonsei University, Seoul 120-749, Korea

${ }^{\S}$ Department of Materials Science \& Engineering, National University of Singapore, 119260 , Singapore

${ }^{\|}$College of Chemistry and Chemical Engineering, Hunan University, Changsha, 410082, Peoples Republic of China

\section{Table of Contents}

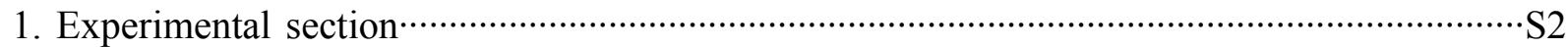

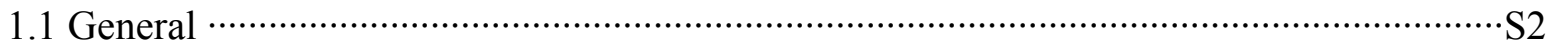

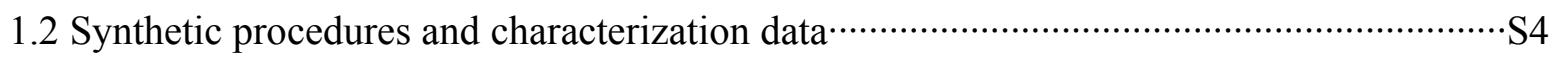

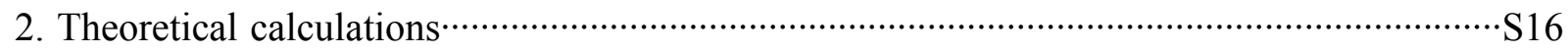

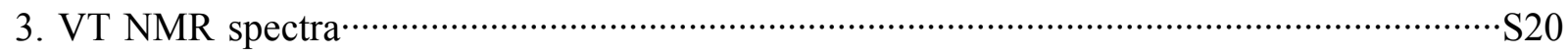

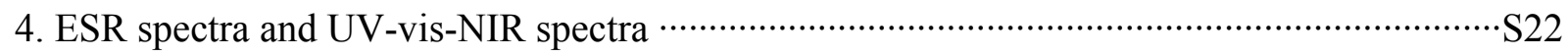

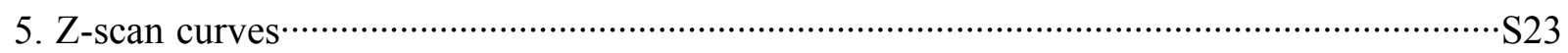

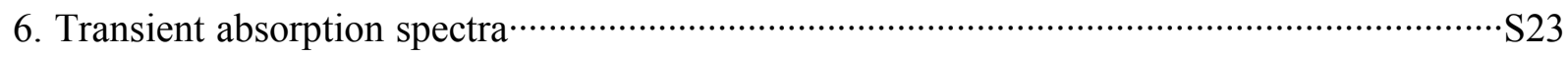

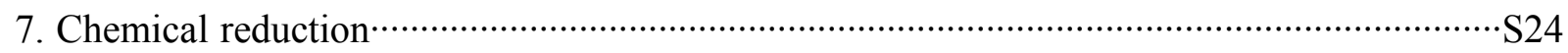

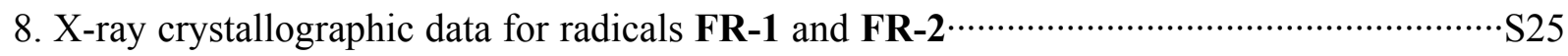

9. Appendix: ${ }^{1} \mathrm{H},{ }^{13} \mathrm{C}$ NMR and HR mass spectra of new compounds …….............................S8

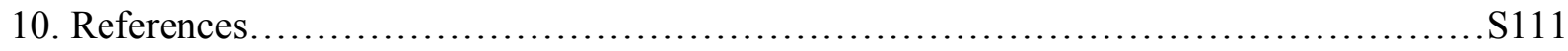




\section{Experimental Section}

\subsection{General}

All reagents and starting materials were obtained from commercial suppliers and used without further purification. Anhydrous dichloromethane (DCM) was distilled from $\mathrm{CaH}_{2}$. Anhydrous toluene and THF were distilled from sodium-benzophenone immediately prior to use. The ${ }^{1} \mathrm{H}$ NMR and ${ }^{13} \mathrm{C}$ NMR spectra were recorded in solution of $\mathrm{CDCl}_{3}$ or $\mathrm{CD}_{2} \mathrm{Cl}_{2}$ on Bruker DPX300/DPX500 NMR spectrometer with tetramethylsilane (TMS) as the internal standard. The following abbreviations were used to explain the multiplicities: $\mathrm{s}=$ singlet, $\mathrm{d}=$ doublet, $\mathrm{t}=$ triplet, $\mathrm{m}=$ multiplet. HR-APCI mass spectra (MS) were recorded on a Bruker amazon instrument. EI mass spectra were recorded on Agilent5975C DIP/MS mass spectrometer. The electrochemical measurements were carried out in anhydrous DCM with $0.1 \mathrm{M}$ tetrabutylammonium hexafluorophosphate $\left(n-\mathrm{Bu}_{4} \mathrm{NPF}_{6}\right)$ as the supporting electrolyte at room temperature under the protection of nitrogen. A gold stick was used as working electrode, platinum wire was used as counting electrode, and $\mathrm{Ag} / \mathrm{AgCl}(3 \mathrm{M} \mathrm{KCl}$ solution) as reference electrode. The potential was externally calibrated against the ferrocene/ferrocenium couple. Steady-state UV-vis absorption were recorded on a Shimadzu UV-1700 and UV-3600 spectrometer. Continuous wave X-band ESR spectra were obtained with a JEOL (FA200) spectrometer.

A Quantum Design 7 Tesla SQUID-VSM system was used for the magnetic measurements in this work. A powder sample with a weight of 5-10 mg was sealed in a plastic capsule. The magnetic susceptibility was measured in the temperature range of 2 to $380 \mathrm{~K}$ with an applied field of $0.5 \mathrm{~T}$. After correction of diamagnetic contributions from the sample, using tabulated constants, sample holder and paramagnetic contamination, the magnetic data were fitted with Bleaney-Bowers equation for FR-2/4/6,

$$
\chi_{M} T=\frac{2 N \beta^{2} g^{2}}{k_{B}\left[3+\exp \left(-2 J / k_{B} T\right)\right]}
$$

and with the trimer model for $\mathbf{F R - 3 / 5}$ :

$$
\chi_{M} T=\frac{N \beta^{2}}{4 k_{B} T} \frac{g^{2}+g^{2} \exp \left(2 J / k_{B} T\right)+10 g^{2} \exp \left(3 J / k_{B} T\right)}{1+\exp \left(2 J / k_{B} T\right)+2 \exp \left(3 J / k_{B} T\right)}
$$


where, $-2 J$ is correlated to the excitation energy from the singlet ground state to the triplet excited state for FR-2/4/6. And $-3 J$ is the energy gap between the quartet exited state and the doulet ground state for $\mathbf{F R - 3 / 5}$.

The femtosecond time-resolved transient absorption $\left(f_{s}\right.$-TA) spectrometer consists of an optical parametric amplifier (OPA; Palitra, Quantronix) pumped by a Ti: sapphire regenerative amplifier system (Integra-C, Quantronix) operating at $1 \mathrm{kHz}$ repetition rate and an optical detection system. The generated OPA pulses have a pulse width of $\sim 100 \mathrm{fs}$ and an average power of $1 \mathrm{~mW}$ in the range of 280-2700 nm, which are used as pump pulses. White light continuum (WLC) probe pulses were generated using a sapphire window (3 $\mathrm{mm}$ thick) by focusing a small portion of the fundamental $800 \mathrm{~nm}$ pulses which was picked off by a quartz plate before entering the OPA. The time delay between pump and probe beams was carefully controlled by making the pump beam travel along a variable optical delay (ILS250, Newport). Intensities of the spectrally dispersed WLC probe pulses are monitored by a High Speed Spectrometer (Ultrafast Systems) for both visible and near-infrared measurements. To obtain the time-resolved transient absorption difference signal $(\Delta \mathrm{A})$ at a specific time, the pump pulses were chopped at $500 \mathrm{~Hz}$ and absorption spectra intensities were saved alternately with or without pump pulse. Typically, 4000 pulses excite the samples to obtain the fs-TA spectra at each delay time. The polarization angle between pump and probe beam was set at the magic angle $\left(54.7^{\circ}\right)$ using a Glan-laser polarizer with a half-wave retarder in order to prevent polarization-dependent signals. Cross-correlation fwhm in pump-probe experiments was less than 200 fs and chirp of WLC probe pulses was measured to be $800 \mathrm{fs}$ in the $400-800 \mathrm{~nm}$ region. To minimize chirp, all reflection optics in the probe beam path and a quartz cell of $2 \mathrm{~mm}$ path length were used. After fs-TA experiments, the absorption spectra of all compounds were carefully examined to detect if there were artifacts due to degradation and photo-oxidation of samples. The three-dimensional data sets of $\Delta \mathrm{A}$ versus time and wavelength were subjected to singular value decomposition and global fitting to obtain the kinetic time constants and their associated spectra using Surface Xplorer software (Ultrafast Systems).

The two-photon absorption spectrum was measured in the NIR region using the open-aperture Z-scan method with 130 fs pulses from an optical parametric amplifier (Light Conversion, TOPAS) operating at a repetition rate of $1 \mathrm{kHz}$ generated from a Ti: sapphire regenerative 
amplifier system (Spectra-Physics, Hurricane). After passing through a $10 \mathrm{~cm}$ focal length lens, the laser beam was focused and passed through a $1 \mathrm{~mm}$ quartz cell. Since the position of the sample cell could be controlled along the laser beam direction ( $z$ axis) using the motor controlled delay stage, the local power density within the sample cell could be simply controlled under constant laser intensity. The transmitted laser beam from the sample cell was then detected by the same photodiode as used for reference monitoring. The on-axis peak intensity of the incident pulses at the focal point, $I_{0}$, ranged from 40 to $60 \mathrm{GW} \mathrm{cm}^{-2}$. For a Gaussian beam profile, the nonlinear absorption coefficient can be obtained by curve fitting of the observed open-aperture traces $T(z)$ with the following equation:

$$
T(z)=1-\frac{\beta I_{0}\left(1-e^{-\alpha_{0} l}\right)}{2 \alpha_{0}\left[1+\left(z / z_{0}\right)^{2}\right]}
$$

where $\alpha_{0}$ is the linear absorption coefficient, $l$ is the sample length, and $z 0$ is the diffraction length of the incident beam. After the nonlinear absorption coefficient has been obtained, the TPA cross section $\sigma^{(2)}$ of one solute molecule (in units of GM, where $1 \mathrm{GM}=10^{-50} \mathrm{~cm}^{4} \mathrm{~s}_{\text {photon }}{ }^{-1}$ molecule ${ }^{1}$ ) can be determined by using the following relationship:

$$
\beta=\frac{10^{-3} \sigma^{(2)} N_{A} d}{h v}
$$

Where $N_{\mathrm{A}}$ is the Avogadro constant, $d$ is the concentration of the compound in solution, $h$ is the Planck constant, and $v$ is the frequency of the incident laser beam.

\subsection{Synthetic procedures and characterization data}

\section{Synthesis of compound 2.}

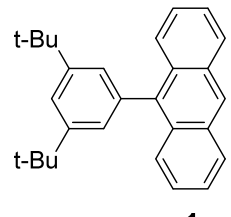

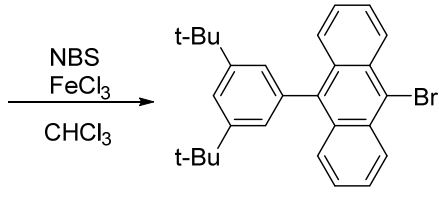

Compound 1 was synthesized according to previously reported literature in our group (J.Am. Chem. Soc. 2012, 134, 14513). Under argon atmosphere, compound 1 (7.50 g, $20.46 \mathrm{mmol})$ was dissolved in a solution of chloroform $(150 \mathrm{~mL})$. To this solution, NBS $(3.64 \mathrm{~g}, 20.46 \mathrm{mmol})$ and $\mathrm{FeCl}_{3}(10 \mathrm{mg})$ were added. The reaction mixture was heated at $70{ }^{\circ} \mathrm{C}$ for $3 \mathrm{~h}$, after cooling to 
room temperature water was added to quench the reaction. The solution was extracted with DCM for three times. The combined organic solution was washed with sodium hydroxide solution and sodium chloride solution and dried over anhydrous sodium sulfate. After removal of the solvent, the residue was purified by flash column chromatography (silica gel, DCM/Hexane = 1:20). Yellow solid 2 was obtained in $89 \%$ yield $(8.10 \mathrm{~g}) .{ }^{1} \mathrm{H}$ NMR (300 MHz, $\left.\mathrm{CDCl}_{3}, \delta \mathrm{ppm}\right): 8.63$ (d, $2 \mathrm{H}, J=8.9 \mathrm{~Hz}), 7.73(\mathrm{~d}, 2 \mathrm{H}, J=8.8 \mathrm{~Hz}), 7.63-7.58(\mathrm{~m}, 3 \mathrm{H}), 7.43-7.37$ (m, 2H), 7.27 (d, 2H, $J=$ $1.8 \mathrm{~Hz}), 1.41(\mathrm{~s}, 18 \mathrm{H}) .{ }^{13} \mathrm{C} \mathrm{NMR}\left(75 \mathrm{MHz}, \mathrm{CDCl}_{3}, \delta \mathrm{ppm}\right): 150.7,139.3,137.3,131.2,130.3$, $127.8,127.7,126.8,125.5,125.4,122.3,121.2,35.0,31.6$. HRMS (APCI, $m / z):\left[(\mathrm{M}+\mathrm{H})^{+}\right]$calcd for $\mathrm{C}_{28} \mathrm{H}_{30} \mathrm{Br}$, 445.1525; found, 445.1521.

\section{Synthesis of compound 3 .}
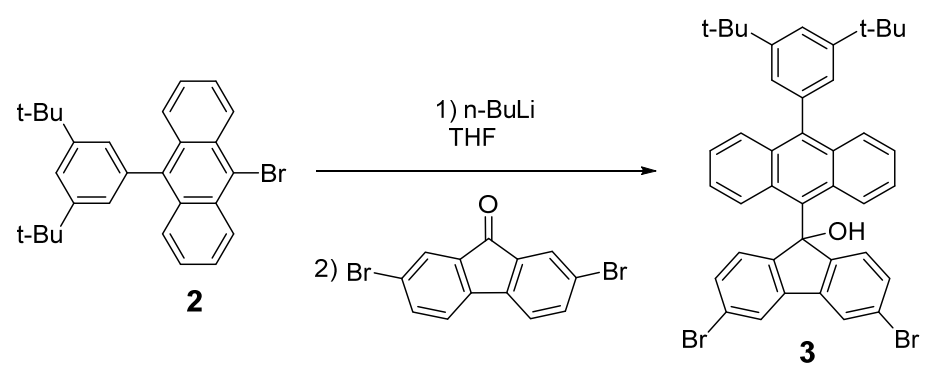

Under argon atmosphere, a solution of compound $2(3.50 \mathrm{~g}, 7.86 \mathrm{mmol})$ in dry THF $(150 \mathrm{~mL})$ was cooled to $-78{ }^{\circ} \mathrm{C}$ and then $n$-BuLi (1.6 M in $n$-hexane, $\left.4.80 \mathrm{~mL}, 7.68 \mathrm{mmol}\right)$ was added slowly. The solution was stirred at $-78^{\circ} \mathrm{C}$ for $1 \mathrm{~h}$ and then 3,6-dibromo- $9 H$-fluoren-9-one $(2.00 \mathrm{~g}$, $5.92 \mathrm{mmol}$ ) was added, the mixture was slowly warmed to room temperature and stirred overnight. The reaction was quenched by water and extracted by diethyl ether for three times. The combined organic solution was washed with sodium chloride solution and dried over anhydrous sodium sulfate. After removal of the solvent, the residue was purified by flash column chromatography (silica gel, DCM/Hexane $=1: 2$ ). Yellow solid 3 was obtained in $81 \%$ yield (3.38 g). ${ }^{1} \mathrm{H}$ NMR (300 MHz, $\mathrm{CDCl}_{3}, \delta$ ppm): 9.57 (d, $1 \mathrm{H}, J=9.2 \mathrm{~Hz}$ ), 7.90 (d, 2H, $J=1.7 \mathrm{~Hz}$ ), $7.64(\mathrm{~d}, 1 \mathrm{H}, J=8.1 \mathrm{~Hz}), 7.52-7.46(\mathrm{~m}, 2 \mathrm{H}), 7.43-7.37$ (m, 1H), 7.28-7.24 (m, 3H), 7.16-7.09 (m, 4H), 7.00-6.94 (m, 1H), $6.84(\mathrm{~d}, 1 \mathrm{H}, J=9.0 \mathrm{~Hz}), 6.75-6.69(\mathrm{~m}, 1 \mathrm{H}), 2.72(\mathrm{~s}, 1 \mathrm{H}), 1.29(\mathrm{~s}, 18 \mathrm{H})$. ${ }^{13} \mathrm{C}$ NMR (75 MHz, $\mathrm{CD}_{2} \mathrm{Cl}_{2}, \delta \mathrm{ppm}$ ): 151.5, 150.7, 140.7, 139.7, 137.9, 132.0, 131.6, 131.2, 131.1, 130.0, 128.1, 127.9, 127.8, 127.6, 126.1, 125.3, 124.6, 124.2, 124.0, 123.9, 123.1, 121.2, 86.2, 34.8, 31.2. HRMS (APCI, $m / z)$ : [(M-H)`] calcd for $\mathrm{C}_{41} \mathrm{H}_{35} \mathrm{Br}_{2} \mathrm{O}, 701.1060$; found, 701.1065. 


\section{Synthesis of compound 4 .}
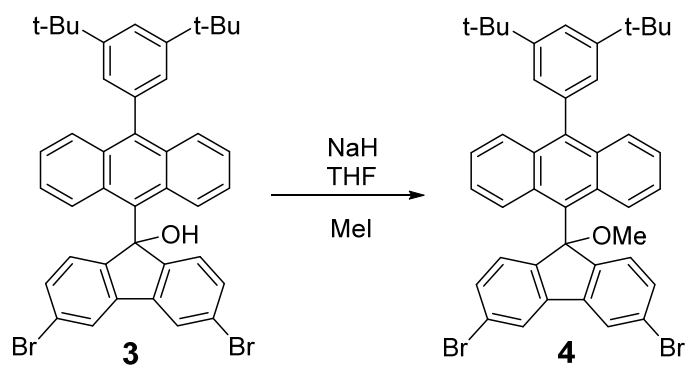

Under argon atmosphere, a solution of compound 3 (3.00 g, $4.26 \mathrm{mmol})$ in dry THF (15 mL) was added slowly to a suspension of sodium hydride (60\% oil suspension, $511 \mathrm{mg}, 12.78 \mathrm{mmol}$ ) in dry THF $(100 \mathrm{~mL})$ at $0{ }^{\circ} \mathrm{C}$. The mixture was stirred for $30 \mathrm{~min}$ and then methyl iodide $(2.0 \mathrm{M}$ in tert-butyl methyl ether, $7.52 \mathrm{~mL}, 17.04 \mathrm{mmol}$ ) was added dropwise, the mixture was slowly warmed to room temperature and stirred overnight. The reaction was quenched by water and extracted by diethyl ether for three times. The combined organic solution was washed with sodium chloride solution and dried over anhydrous sodium sulfate. After removal of the solvent, the residue was purified by flash column chromatography (silica gel, Chloroform/Hexane = 1:8). Yellow solid 4 was obtained in 95\% yield (2.91 g). ${ }^{1} \mathrm{H}$ NMR (300 MHz, $\mathrm{CDCl}_{3}, \delta$ ppm): 9.49 (d, $1 \mathrm{H}, J=9.2 \mathrm{~Hz}), 7.97$ (d, 2H, $J=1.7 \mathrm{~Hz}), 7.74$ (d, 1H, $J=8.2 \mathrm{~Hz}), 7.60-7.49$ (m, 3H), 7.38-7.33 (m, 3H), 7.26-7.23 (m, 4H), 7.10-7.04 (m, 1H), 6.92 (d, 1H, $J=9.0 \mathrm{~Hz}), 6.82-6.77$ (m, 1H), 2.95 (s, 3H), 1.38 (s, 18H). $\left.{ }^{13} \mathrm{C} \mathrm{NMR} \mathrm{(75} \mathrm{MHz,} \mathrm{CDCl}_{3}, \delta \mathrm{ppm}\right): 150.6,147.3,141.1,140.8,138.0$, $131.7,131.5,131.4,130.4,130.2$, 128.4, 128.1, 127.8, 127.7, 125.5, 125.4, 124.5, 124.2, 124.0, 123.9, 123.7, 123.4, 121.0, 90.9, 50.8, 35.0, 31.6. HRMS (APCI, $m / z$ ): [M] calcd for $\mathrm{C}_{42} \mathrm{H}_{38} \mathrm{Br}_{2} \mathrm{O}$, 716.1284 ; found, 716.1280 .

\section{Synthesis of compounds 5 and 6.}
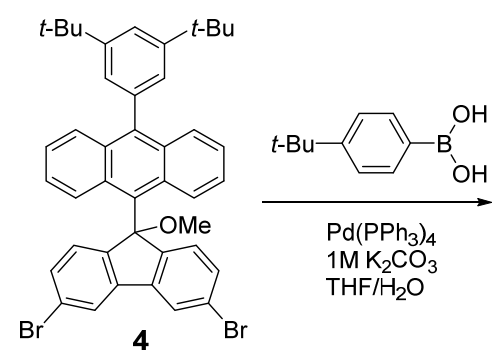

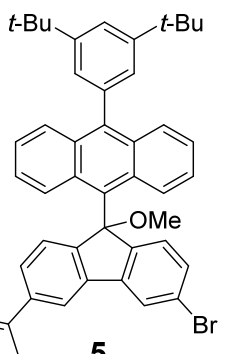

5

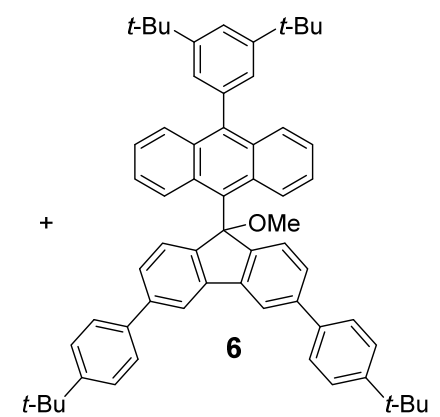


Under argon atmosphere, a mixture of compound 4 (600 $\mathrm{mg}, 0.83 \mathrm{mmol})$, 4-tertbutylbenzeneboronic acid (134 mg, $0.75 \mathrm{mmol}), \mathrm{Pd}\left(\mathrm{PPh}_{3}\right)_{4}(23 \mathrm{mg}, 0.02 \mathrm{mmol})$ and $\mathrm{K}_{2} \mathrm{CO}_{3}(552$ $\mathrm{mg}, 4.00 \mathrm{mmol})$ in a solution of water $(4 \mathrm{~mL})$, and THF $(35 \mathrm{~mL})$ was stirred and heated at $80^{\circ} \mathrm{C}$ for $12 \mathrm{~h}$. The mixture was extracted with DCM for two times. The combined organic solution was washed with sodium chloride solution and dried by anhydrous sodium sulfate. After removal of the solvent, the residue was purified by flash column chromatography (silica gel, DCM/Hexane = 1:4). Yellow solid 5 was obtained in 53\% $(310 \mathrm{mg}) .{ }^{1} \mathrm{H}$ NMR $(300 \mathrm{MHz}$, $\left.\mathrm{CDCl}_{3}, \delta \mathrm{ppm}\right): 9.59$ (d, $\left.1 \mathrm{H}, J=9.2 \mathrm{~Hz}\right), 8.06-8.04$ (m, 2H), 7.74 (d, $\left.1 \mathrm{H}, J=8.5 \mathrm{~Hz}\right), 7.66-7.63$ $(\mathrm{m}, 2 \mathrm{H}), 7.60-7.51(\mathrm{~m}, 5 \mathrm{H}), 7.47(\mathrm{~s}, 2 \mathrm{H}), 7.39-7.31(\mathrm{~m}, 2 \mathrm{H}), 7.26-7.22(\mathrm{~m}, 3 \mathrm{H}), 7.09-7.03$ (m, $2 \mathrm{H}), 6.80-6.75(\mathrm{~m}, 1 \mathrm{H}), 3.01(\mathrm{~s}, 3 \mathrm{H}), 1.40-1.39(\mathrm{~m}, 27 \mathrm{H}) .{ }^{13} \mathrm{C} \mathrm{NMR}\left(75 \mathrm{MHz}, \mathrm{CDCl}_{3}, \delta \mathrm{ppm}\right)$ : $150.9,150.6,150.6,147.7,147.1,142.5,142.4,140.6,139.8,138.3,137.8,131.7,131.5,131.4$, $131.0,130.3,128.6,128.2$, 128.1, 127.8, 127.6, 127.6, 126.8, 126.6, 125.9, 125.9, 125.6, 125.5, $124.3,124.2,123.9,123.8,123.5,123.2,120.9,119.2,91.2,50.8,35.0,34.6,31.6,31.4$. HRMS (APCI, m/z): [M] calcd for $\mathrm{C}_{52} \mathrm{H}_{51} \mathrm{BrO}, 770.3118$; found, 770.3111. Yellow solid 6 was obtained in $20 \%$ yield $(125 \mathrm{mg}) .{ }^{1} \mathrm{H}$ NMR (300 MHz, $\left.\mathrm{CDCl}_{3}, \delta \mathrm{ppm}\right): 9.68(\mathrm{~d}, 1 \mathrm{H}, J=9.1 \mathrm{~Hz}), 8.14-8.13$ (m, 2H), $7.75(\mathrm{~d}, 1 \mathrm{H}, J=8.1 \mathrm{~Hz}), 7.69-7.66(\mathrm{~m}, 4 \mathrm{H}), 7.60-7.51(\mathrm{~m}, 7 \mathrm{H}), 7.45(\mathrm{~m}, 4 \mathrm{H}), 7.40-7.35$ (m, 1H), 7.27-7.26 (m, 2H), 7.16 (d, 1H, J=9.1 Hz), 7.07-7.02 (m, 1H), 6.77-6.71 (m, 1H), 3.06 $(\mathrm{s}, 3 \mathrm{H}), 1.40-1.39(\mathrm{~m}, 36 \mathrm{H}) .{ }^{13} \mathrm{C} \mathrm{NMR}\left(75 \mathrm{MHz}, \mathrm{CDCl}_{3}, \delta \mathrm{ppm}\right): 150.7,150.6,147.5,142.2$, 141.0, 140.4, 138.5, 138.1, 132.2, 131.7, 131.5, 130.4, 128.8, 128.5, 128.1, 127.5, 127.2, 126.9, $126.5,126.3,125.8,125.6,124.2,123.9,123.3,120.9,119.0,91.4,50.9,35.0,34.6,31.7,31.4$. HRMS (ESI, $m / z)$ : [(M+Na) $\left.{ }^{+}\right]$calcd for $\mathrm{C}_{62} \mathrm{H}_{64} \mathrm{NaO}, 847.4863$; found, 847.4875.

\section{Synthesis of radical FR-1.}
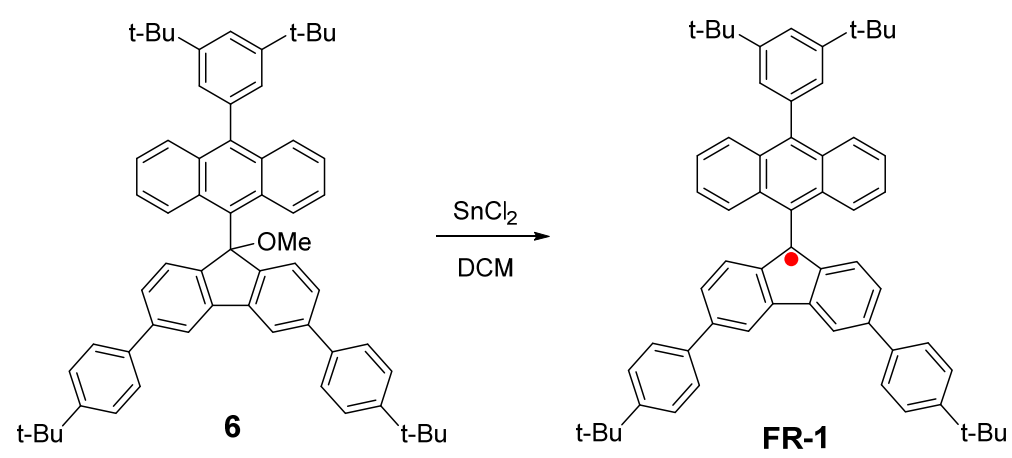
Under argon atmosphere, compound 6 (100 $\mathrm{mg}, 0.12 \mathrm{mmol})$ was dissolved in a solution of DCM (35 mL), then $\mathrm{SnCl}_{2}(115 \mathrm{mg}, 0.61 \mathrm{mmol})$ was added. The mixture was stirred at room temperature overnight. The solvent was removed under reduced pressure at room temperature and the residue was purified by flash column chromatography (silica gel, DCM/Hexane = 1:4). Black solid monoradical FR-1 was obtained in 90\% yield $(87 \mathrm{mg})$. No NMR spectrum can be recorded due to the paramagnetic property. MALDI-TOF Mass: $m / z$ 793.2, calc. 793.5. HRMS (APCI, $m / z):\left[(\mathrm{M}+\mathrm{H})^{+}\right]$calcd for $\mathrm{C}_{61} \mathrm{H}_{62}, 794.4846$; found, 794.4850.

\section{Synthesis of compound 7.}

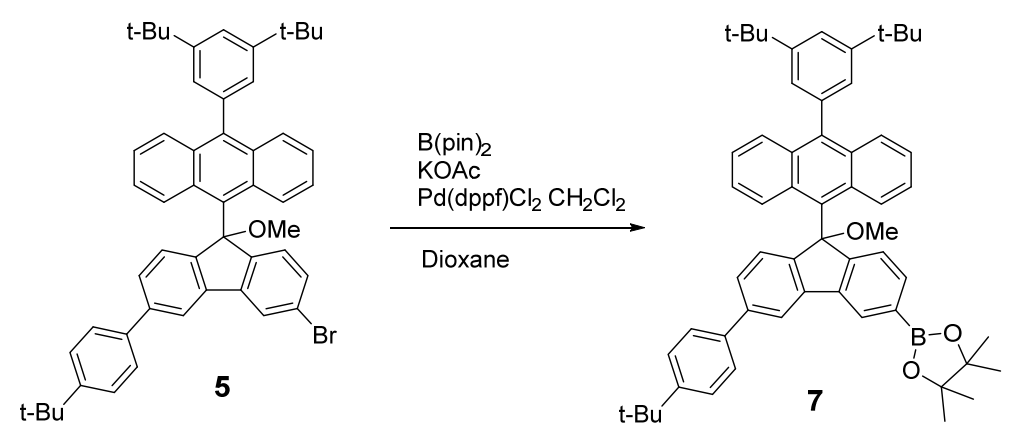

A mixture of 5 (420 mg, $0.54 \mathrm{mmol})$, bis(pinacolato)diboron (152 mg, $0.60 \mathrm{mmol})$, [1, 1'bis(diphenylphosphino)ferrocene]palladium(II) dichloride dichloromethane adduct (16 mg, 0.02 mmol) and potassium acetate $(162 \mathrm{mg}, 1.65 \mathrm{mmol})$ in a solution of dioxane $(20 \mathrm{~mL})$ under argon atmosphere was stirred and heated at $85{ }^{\circ} \mathrm{C}$ for $8 \mathrm{~h}$. The solvent was removed under reduced pressure and the residue was purified by flash column chromatography (silica gel, DCM/Hexane = 1:2). Yellow solid 7 was obtained in $75 \%$ yield $(340 \mathrm{mg}) .{ }^{1} \mathrm{H} \mathrm{NMR}\left(300 \mathrm{MHz}, \mathrm{CDCl}_{3}, \delta \mathrm{ppm}\right)$ : $9.66(\mathrm{~d}, 1 \mathrm{H}, J=9.2 \mathrm{~Hz}), 8.40(\mathrm{~s}, 1 \mathrm{H}), 8.19(\mathrm{~s}, 1 \mathrm{H}), 7.78-7.67(\mathrm{~m}, 4 \mathrm{H}), 7.61-7.44(\mathrm{~m}, 7 \mathrm{H}), 7.41-$ 7.37 (m, 2H), 7.30-7.28 (m, 2H), $7.12(\mathrm{~d}, 1 \mathrm{H}, J=9.1 \mathrm{~Hz}), 7.08-7.03(\mathrm{~m}, 1 \mathrm{H}), 6.78-6.72(\mathrm{~m}, 1 \mathrm{H})$, $3.01(\mathrm{~s}, 3 \mathrm{H}), 1.44-1.41$ (m, 39H). ${ }^{13} \mathrm{C} \mathrm{NMR}\left(75 \mathrm{MHz}, \mathrm{CDCl}_{3}, \delta \mathrm{ppm}\right): 151.9,150.5,150.5,150.4$, $146.5,142.0,141.0,140.2,139.7,138.3,137.9,135.0,132.0,131.5,131.4,130.1,128.6,128.3$, $127.9,127.3,127.0,126.7,126.6,126.4,126.1,125.7,125.6,125.5,124.1,123.8,123.3,120.8$, $119.1,91.5,84.0,50.6,34.9,34.5,31.6,31.3,24.9$. HRMS (APCI, $m / z$ ): [M] calcd for $\mathrm{C}_{58} \mathrm{H}_{63} \mathrm{BO}_{3}, 818.4874$; found, 818.4871.

\section{Synthesis of compound 8 .}




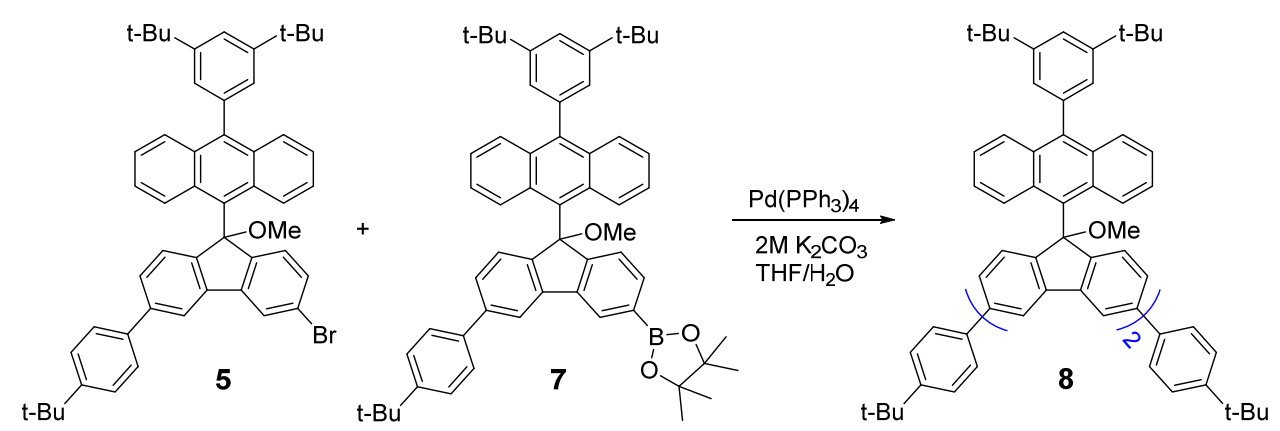

Under argon atmosphere, a mixture of $5(77 \mathrm{mg}, 0.10 \mathrm{mmol}), 7(82 \mathrm{mg}, 0.10 \mathrm{mmol}), \mathrm{Pd}\left(\mathrm{PPh}_{3}\right)_{4}$ (23 mg, $0.02 \mathrm{mmol})$ and $\mathrm{K}_{2} \mathrm{CO}_{3}(0.55 \mathrm{~g}, 4.00 \mathrm{mmol})$ in a solution of water $(2 \mathrm{~mL})$, and THF (10 $\mathrm{mL}$ ) was stirred and heated at $80{ }^{\circ} \mathrm{C}$ overnight. After cooling, the mixture was extracted with DCM for two times. The combined organic solution was washed with sodium chloride solution and dried by anhydrous sodium sulfate. After removal of the solvent, the residue was purified by flash column chromatography (silica gel, DCM/Hexane $=1: 3$ ). Yellow solid 8 was obtained in $61 \%$ yield (85 mg). ${ }^{1} \mathrm{H}$ NMR (300 MHz, $\mathrm{CDCl}_{3}, \delta \mathrm{ppm}$ ): 9.72 (d, $2 \mathrm{H}, J=9.2 \mathrm{~Hz}$ ), 8.31-8.24 (m, 4H), $7.79(\mathrm{~d}, 2 \mathrm{H}, J=8.7 \mathrm{~Hz}), 7.74-7.72(\mathrm{~m}, 4 \mathrm{H}), 7.65-7.49(\mathrm{~m}, 18 \mathrm{H}), 7.44-7.39$ (m, 2H), 7.31 (d, $4 \mathrm{H}, J=1.7 \mathrm{~Hz}), 7.23$ (d, 2H, $J=9.2 \mathrm{~Hz}), 7.12-7.07(\mathrm{~m}, 2 \mathrm{H}), 6.84-6.79(\mathrm{~m}, 2 \mathrm{H}), 3.11(\mathrm{~s}, 6 \mathrm{H})$, 1.42 (m, 54H). ${ }^{13} \mathrm{C}$ NMR (75 MHz, $\mathrm{CDCl}_{3}, \delta \mathrm{ppm}$ ): 150.6, 150.5, 148.0, 147.3, 142.2, 142.1, $141.0,140.7,140.3,138.3,138.0,132.0,131.6,131.4,130.2,128.6,128.3,128.0,127.4,127.4$, $127.3,126.8,126.5,126.4,126.1,125.8,125.6,125.5,124.2,123.8,123.3,120.8,119.2,119.1$, 91.3, 77.4, 77.0, 76.6, 50.8, 35.0, 34.6, 31.6, 31.3. HRMS (ESI, $m / z):\left[(\mathrm{M}+\mathrm{Na})^{+}\right]$calcd for $\mathrm{C}_{104} \mathrm{H}_{102} \mathrm{NaO}_{2}, 1405.7772$; found, 1405.7779 .

\section{Synthesis of radical FR-2.}
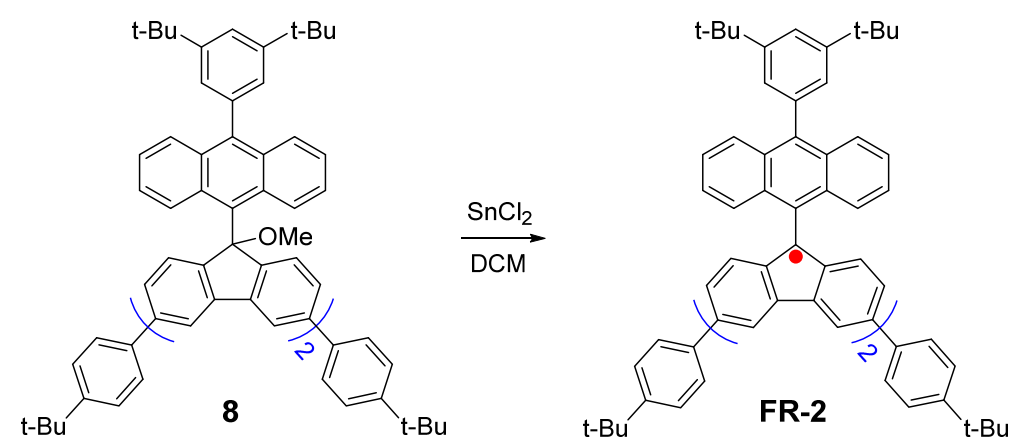

Radical FR-2 was synthesized similarly as described for synthesis of radical FR-1, black solid, yield $82 \%$ (40 mg). ${ }^{1} \mathrm{H}$ NMR (500 MHz, THF- $d_{8}, 213 \mathrm{~K}, \delta$ ppm): 9.17 (s, 1H), 8.95 (s, 1H), 
8.58-8.52 (m, 2H), $8.06(\mathrm{~s}, 4 \mathrm{H})$, 7.95-7.46 (m, 30H), 6.89-6.80 (m, 4H), 1.50-1.37 (m, 54H). MALDI-TOF Mass: $m / z$ 1320.4, calc. 1320.8. HRMS (APCI, $m / z)$ : [(M+H) $\left.{ }^{+}\right]$calcd for $\mathrm{C}_{102} \mathrm{H}_{97}$, 1321.7585; found, 1321.7576 .

\section{Synthesis of compounds 9 and 10.}
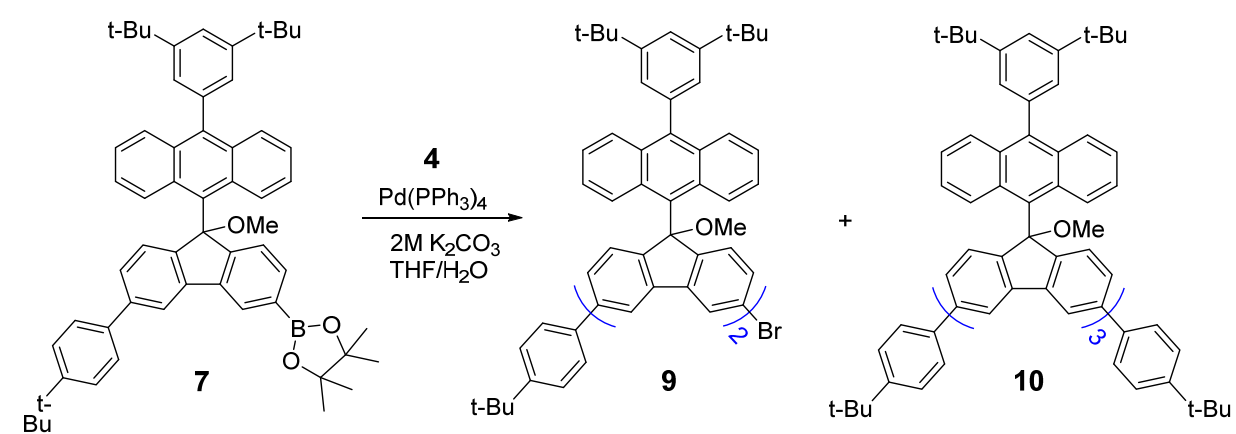

The synthesis is very similar to that for $\mathbf{5}$ and $\mathbf{6}$. Yellow solid $\mathbf{9}$ was obtained in $30 \%$ yield (150 mg). ${ }^{1} \mathrm{H}$ NMR (300 MHz, $\left.\mathrm{CDCl}_{3}, \delta \mathrm{ppm}\right)$ : 9.78-9.67 (m, 2H), 8.35-8.22 (m, 4H), 7.86-7.84 (m, 2H), 7.77 (d, 2H, $J=4.9 \mathrm{~Hz}), 7.70-7.55(\mathrm{~m}, 14 \mathrm{H}), 7.47-7.42$ (m, 3H), 7.37-7.35 (m, 5H), 7.29 (d, $1 \mathrm{H}, J=5.5 \mathrm{~Hz}), 7.19-7.14(\mathrm{~m}, 3 \mathrm{H}), 6.91-6.85(\mathrm{~m}, 2 \mathrm{H}), 3.16-3.11(\mathrm{~m}, 6 \mathrm{H}), 1.48-1.47(\mathrm{~m}, 45 \mathrm{H})$. ${ }^{13} \mathrm{C}$ NMR (75 MHz, $\left.\mathrm{CDCl}_{3}, \delta \mathrm{ppm}\right): 150.8,150.7,148.4,147.8,147.8,147.4,142.5,142.4$, $141.9,141.3,140.8,140.7,140.5,140.0,138.5,138.3,138.0,132.1,131.8,131.8,131.6,131.3$, $130.4,130.4,128.8,128.7,128.4,128.2$, 128.1, 127.7, 127.6, 127.4, 126.9, 126.8, 126.7, 126.6, $126.3,125.9,125.7,125.6,124.4,124.3,124.0,123.7,123.4,123.4,121.0,121.0,119.6,119.3$, $119.2,91.5,91.3,50.9,50.9,35.1,34.7,31.7,31.5$. HRMS (APCI, $m / z)$ : [M] calcd for $\mathrm{C}_{94} \mathrm{H}_{89} \mathrm{BrO}_{2}$, 1328.6040; found, 1328.6047. Yellow solid 10 was obtained in $16 \%$ yield $(110 \mathrm{mg})$. ${ }^{1} \mathrm{H}$ NMR (300 MHz, $\mathrm{CDCl}_{3}, \delta$ ppm): 9.83-9.78 (m, 3H), 8.47 (s, 2H), $8.41(\mathrm{~s}, 2 \mathrm{H}), 8.32(\mathrm{~s}, 2 \mathrm{H})$, 7.91-7.86 (m, 3H), 7.79-7.57 (m, 29H), 7.52-7.46 (m, 3H), 7.41-7.39 (m, 6H), 7.35-7.31 (m, 3H), 7.22-7.14 (m, 3H), 6.97-6.87 (m, 3H), 3.22-3.19 (m, 9H), 1.51-1.50 (m, 54H), $1.45(\mathrm{~s}, 18 \mathrm{H}) .{ }^{13} \mathrm{C}$ NMR (75 MHz, $\left.\mathrm{CDCl}_{3}, \delta \mathrm{ppm}\right): 150.7,150.6,148.1,148.0,147.3,142.3,142.2,142.1,141.2$, $141.0,140.8,140.4,138.4,138.0,132.1,132.0,131.7,131.5,130.3,128.7,128.4,128.1,127.7$, $127.5,127.3,126.9,126.6,126.6,126.2,125.8,125.6,124.3,123.9,123.5,123.4,120.9,119.5$, 119.4, 119.2, 91.4, 50.9, 35.0, 34.6, 31.7, 31.4. HRMS (APCI, $m / z$ ): [M] calcd for $\mathrm{C}_{146} \mathrm{H}_{140} \mathrm{O}_{3}$, 1941.0797; found, 1941.0789. 


\section{Synthesis of radical FR-3.}
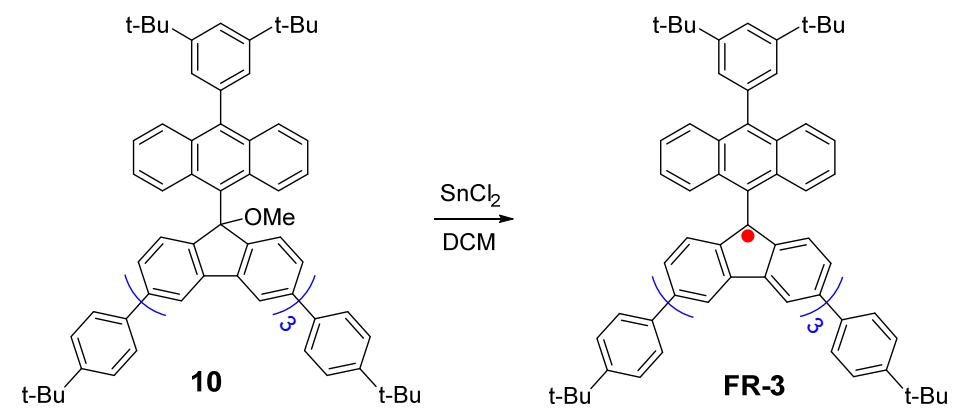

Radical FR-3 was synthesized similarly as described for synthesis of radical FR-1, black solid, yield 75\% (50 mg). MALDI-TOF Mass: $m / z$ 1948.4, calc. 1948.3. HRMS (APCI, $m / z):\left[(\mathrm{M}+\mathrm{H})^{+}\right]$ calcd for $\mathrm{C}_{143} \mathrm{H}_{132}, 1849.0324$; found, 1849.0312 .

\section{Synthesis of compound 11.}
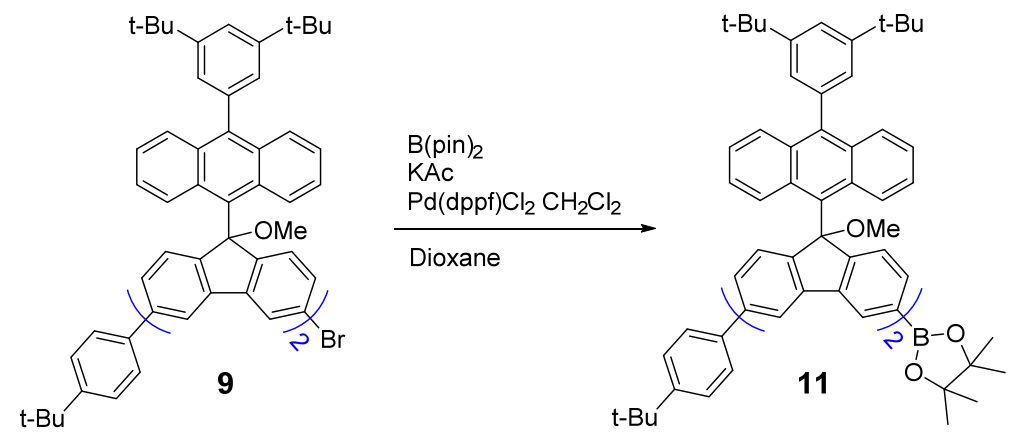

The synthesis is very similar to that for 7 . Yellow solid 11 was obtained in $72 \%$ yield $(65 \mathrm{mg})$. ${ }^{1} \mathrm{H}$ NMR (300 MHz, $\mathrm{CDCl}_{3}, \delta$ ppm): 9.76-9.69 (m, 2H), 8.51 (s, 1H), 8.38-8.31 (m, 3H), 7.83$7.76(\mathrm{~m}, 5 \mathrm{H})$, 7.68-7.52 (m, 14H), 7.47-7.40 (m, 3H), 7.34 (m, 4H), 7.28-7.25 (m, 1H), 7.21-7.08 $(\mathrm{m}, 3 \mathrm{H}), 6.87-6.79(\mathrm{~m}, 2 \mathrm{H}), 3.14-3.07(\mathrm{~m}, 6 \mathrm{H}), 1.47-1.45(\mathrm{~m}, 57 \mathrm{H}) .{ }^{13} \mathrm{C} \mathrm{NMR}\left(75 \mathrm{MHz}, \mathrm{CDCl}_{3}\right.$, $\delta$ ppm): 152.0, 150.6, 150.5, 148.0, 147.2, 142.2, 142.1, 142.0, 141.3, 141.1, 140.8, 140.3, 139.6, 138.3, 138.3, 138.0, 135.2, 132.1, 132.0, 131.6, 131.6, 131.4, 131.4, 130.2, 128.7, 128.6, 128.4, $128.0,127.4,127.3,127.2,126.9,126.7,126.5,126.5,126.2,126.1,125.8,125.6,124.2,123.9$, $123.4,123.3,120.8,119.5,119.3,119.1,91.5,91.3,84.1,35.0,34.6,31.6,31.4,25.0$. HRMS (APCI, $m / z$ ): [M] calcd for $\mathrm{C}_{100} \mathrm{H}_{101} \mathrm{BO}_{4}, 1376.7792$; found, 1376.7799 .

\section{Synthesis of compound 12.}




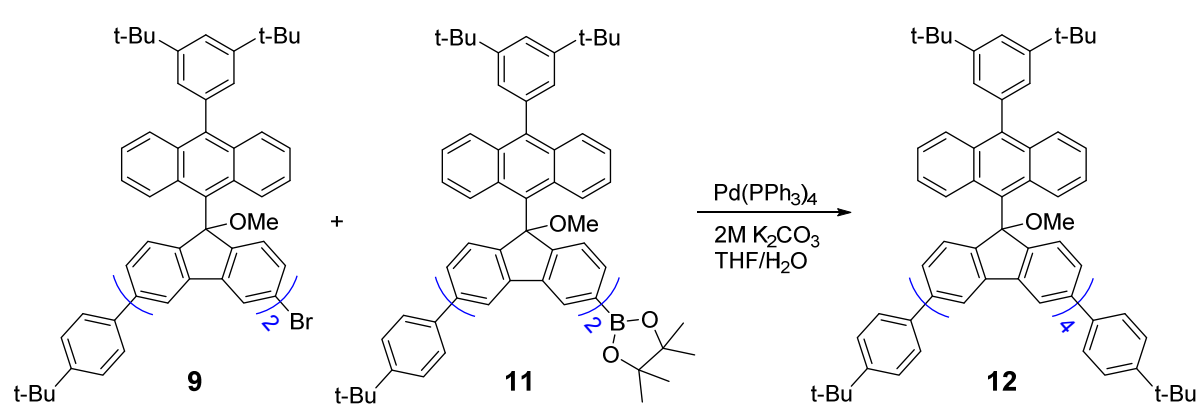

The synthesis is very similar to that for $\mathbf{8}$. Yellow solid 12 was obtained in $54 \%$ yield $(60 \mathrm{mg})$. ${ }^{1} \mathrm{H}$ NMR (300 MHz, $\mathrm{CDCl}_{3}, \delta$ ppm): 9.75-9.67 (m, 4H), 8.36 (s, 4H), 8.30 (s, 2H), 8.21 (s, 2H), 7.83-7.76 (m, 4H), 7.70-7.39 (m, 42H), $7.33(\mathrm{~d}, 4 \mathrm{H}, J=1.6 \mathrm{~Hz}), 7.29(\mathrm{~s}, 4 \mathrm{H}), 7.21$ (d, 2H, $J=$ $9.2 \mathrm{~Hz}), 7.14-7.02(\mathrm{~m}, 4 \mathrm{H}), 6.89-4.84(\mathrm{~m}, 2 \mathrm{H}), 6.80-6.75(\mathrm{~m}, 2 \mathrm{H}), 3.14(\mathrm{~s}, 6 \mathrm{H}), 3.08-3.07$ (m, $6 \mathrm{H}), 1.44-1.37$ (m, 90H). ${ }^{13} \mathrm{C} \mathrm{NMR}\left(75 \mathrm{MHz}, \mathrm{CDCl}_{3}, \delta \mathrm{ppm}\right): 150.6,150.5,148.0,147.9,147.2$, $142.3,142.2$, 142.1 142.0, 141.1, 140.9, 140.7, 140.4, 140.3, 138.3, 137.9, 132.0, 131.9, 131.6, $131.4,130.2$, 128.6, 128.6, 128.3, 128.0, 127.6, 127.4, 127.3, 127.3, 126.8, 126.6, 126.5, 126.4, $126.1,125.8,125.5,124.2,124.1,123.8,123.4,123.3,120.8,119.5,119.4,119.2,119.0,91.3$, 50.8, 35.0, 34.5, 31.6, 31.3. HRMS (APCI, $m / z$ ): [M] calcd for $\mathrm{C}_{188} \mathrm{H}_{178} \mathrm{O}_{4}, 2499.3720$; found, 2499.3711 .

\section{Synthesis of radical FR-4.}
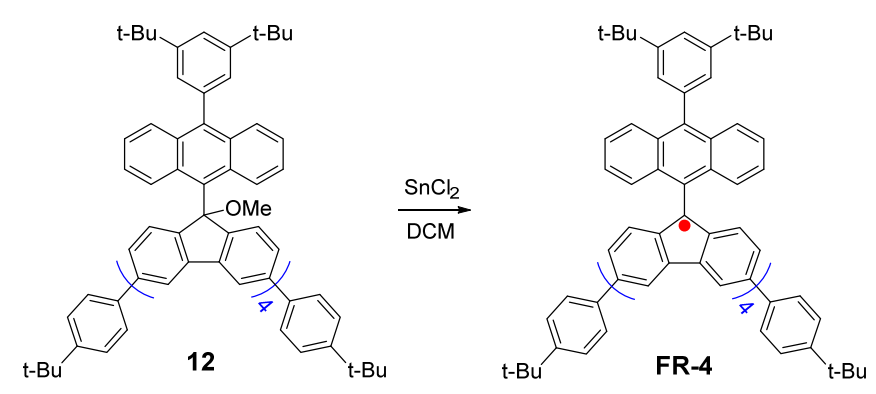

Radical FR-4 was synthesized similarly as described for synthesis of radical FR-1, black solid, yield 71\% (35 mg). MALDI-TOF Mass: $m / z$ 2375.3, calc. 2375.3. HRMS (ESI, $m / z, z=2$ ): [M] calcd for $\mathrm{C}_{184} \mathrm{H}_{166}, 1187.6489$; found, 1187.6486.

\section{Synthesis of compounds 13 and 14.}



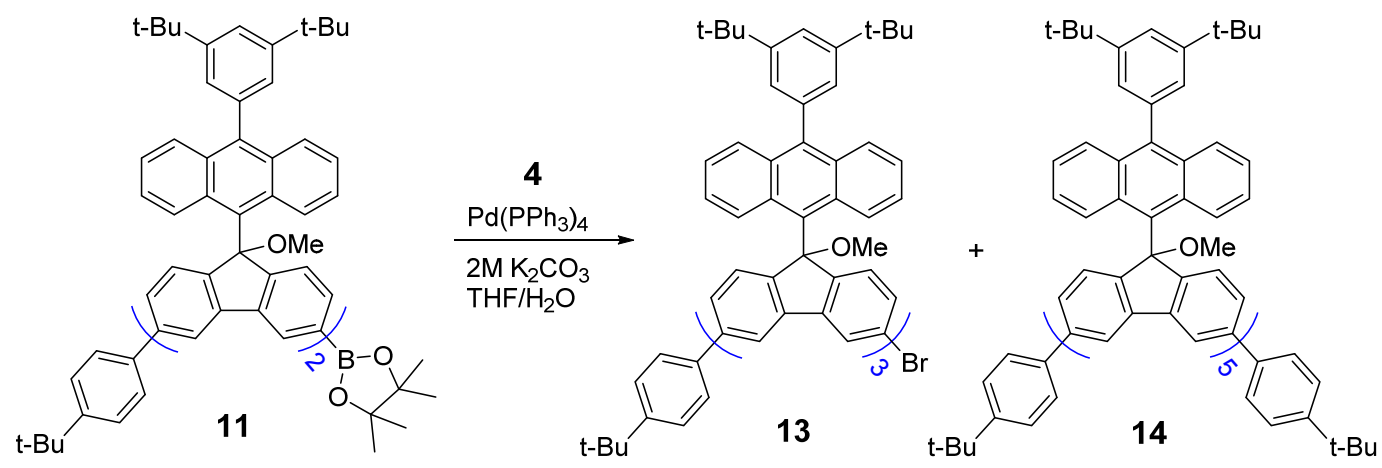

The synthesis is very similar to that for $\mathbf{5}$ and $\mathbf{6}$. Yellow solid $\mathbf{1 3}$ was obtained in $36 \%$ yield (280 mg). ${ }^{1} \mathrm{H}$ NMR (300 MHz, $\mathrm{CDCl}_{3}, \delta$ ppm): 9.79 (d, 2H, $J=9.2 \mathrm{~Hz}$ ), 9.69 (d, $1 \mathrm{H}, J=9.2 \mathrm{~Hz}$ ), 8.46-8.41 (m, 3H), $8.33(\mathrm{~s}, 2 \mathrm{H}), 8.25(\mathrm{~s}, 1 \mathrm{H}), 7.89-7.85(\mathrm{~m}, 3 \mathrm{H}), 7.79$ (d, 2H, $J=8.2 \mathrm{~Hz}), 7.74-$ 7.59 (m, 21H), 7.51-7.30 (m, 13H), 7.20-7.13 (m, 4H), 6.95-6.86 (m, 3H), 3.20-3.18 (m, 6H), $3.12(\mathrm{~s}, 3 \mathrm{H}), 1.49(\mathrm{~m}, 54 \mathrm{H}), 1.46(\mathrm{~s}, 9 \mathrm{H}) .{ }^{13} \mathrm{C} \mathrm{NMR}\left(75 \mathrm{MHz}, \mathrm{CDCl}_{3}, \delta \mathrm{ppm}\right): 150.7,150.6$, 148.2, 148.0, 147.8, 147.6, 147.3, 142.4, 142.2, 142.1, 142.0, 141.2, 141.1, 141.0, 140.8, 140.7, $140.5,140.4,140.0,138.4,138.3,138.2$, 138.0, 132.1, 131.9, 131.7, 131.6, 131.5, 131.2, 130.3, $130.2,128.7,128.6,128.4,128.3,128.1,127.7,127.6,127.5,127.4,126.9,126.7,126.2,126.1$, $125.9,125.6,124.4,124.2,123.9,123.6,123.5,123.4,120.9,119.6,119.5,119.5,119.4,119.2$, 91.4, 91.2, 50.9, 35.0, 34.6, 31.6, 31.4. HRMS (APCI, $m / z)$ : $\left[(\mathrm{M}+\mathrm{H})^{+}\right]$calcd for $\mathrm{C}_{136} \mathrm{H}_{128} \mathrm{BrO}_{3}$, 1887.9041; found, 1887.9050. Yellow solid 14 was obtained in $18 \%$ yield $(100 \mathrm{mg}) .{ }^{1} \mathrm{H}$ NMR (300 MHz, $\left.\mathrm{CDCl}_{3}, \delta \mathrm{ppm}\right)$ : 9.76-9.69 (m, 5H), 8.36-8.31 (m, 8H), 8.22 (s, 2H), 7.84-7.78 (m, 5H), 7.70-7.28 (m, 60H), 7.25-7.21 (m, 3H), 7.14-7.05 (m, 5H), 6.91-6.79 (m, 5H), 3.15-3.09 (m, 15H), $1.46-1.38(\mathrm{~m}, 108 \mathrm{H}) .{ }^{13} \mathrm{C}$ NMR $\left(75 \mathrm{MHz}, \mathrm{CDCl}_{3}, \delta \mathrm{ppm}\right): 150.6,150.5,148.0,147.2$, $142.3,142.3,142.2,142.0,141.1,141.0,140.9,140.7,140.4,140.4,140.3,138.3,137.9,132.0$, $131.9,131.6,131.4,130.2,130.2,128.6,128.3,128.1,128.0,127.6,127.5,127.3,126.8,126.6$, $125.8,125.6,124.2,123.8,123.4,123.3,120.8,119.5,119.4,119.2,119.1,91.3,50.8,35.0,34.5$, 31.6, 31.3. HRMS (APCI, $m / z$ ): [M] calcd for $\mathrm{C}_{230} \mathrm{H}_{216} \mathrm{O}_{5}, 3057.6642$; found, 3057.6627 .

\section{Synthesis of radical FR-5.}



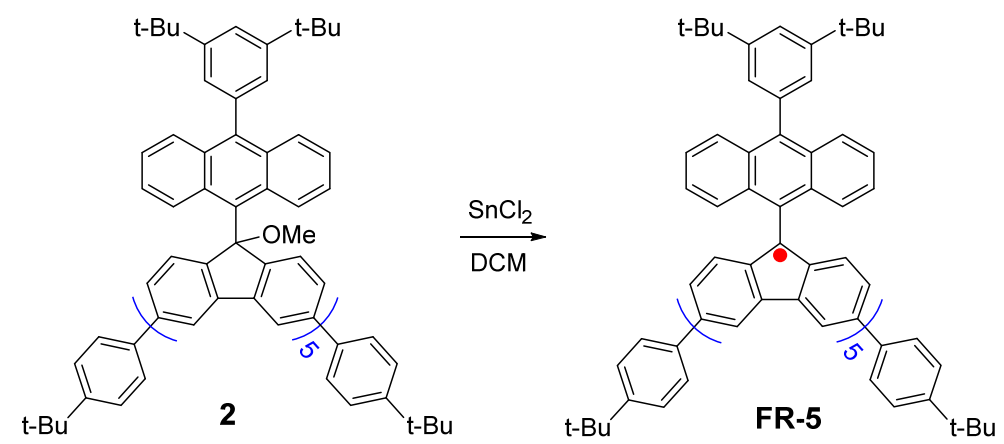

Radical FR-5 was synthesized similarly as described for synthesis of radical FR-1, black solid, yield 79\% (40 mg). MALDI-TOF Mass: $m / z$ 2902.5, calc. 2902.6. HRMS (ESI, $m / z, z=2$ ): [M] calcd for $\mathrm{C}_{225} \mathrm{H}_{201}, 1451.2859$; found, 1451.2854.

\section{Synthesis of compound 15.}
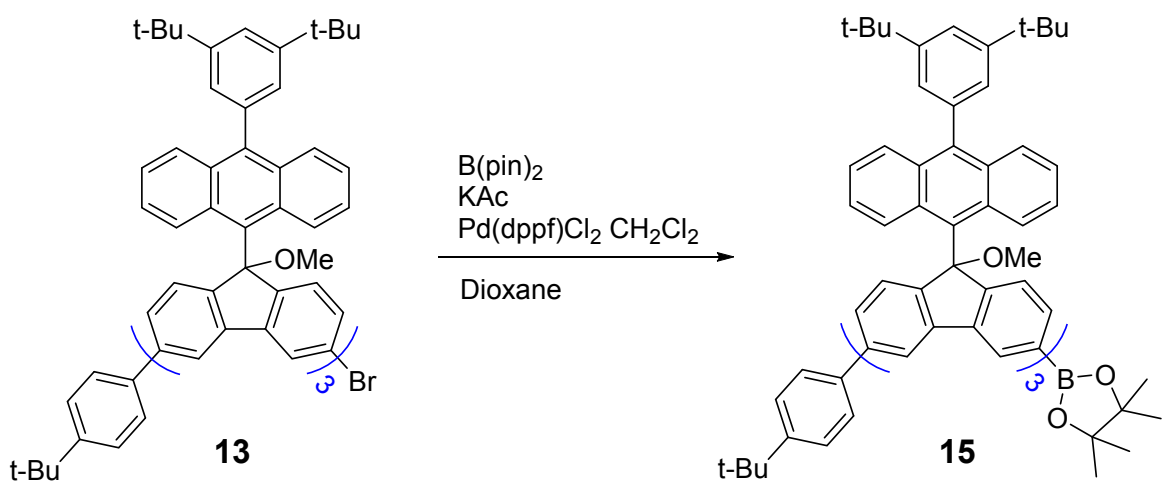

The synthesis is very similar to that for 7 . Yellow solid 15 was obtained in $77 \%$ yield $(130 \mathrm{mg})$. ${ }^{1} \mathrm{H}$ NMR (300 MHz, $\mathrm{CDCl}_{3}, \delta$ ppm): 9.78-9.69 (m, 3H), 8.51 (s, 1H), 8.46-8.37 (m, 4H), 8.28 (s, 1H), 7.86-7.80 (m, 3H), 7.78-7.73 (m, 3H), 7.70-7.54 (m, 20H), 7.45-7.27 (m, 13H), 7.21-7.08 (m, 4H), 6.93-6.80 (m, 3H), 3.18-3.15 (m, 6H), 3.07 (s, 3H), 1.46-1.42 (m, 75H). ${ }^{13} \mathrm{C}$ NMR $(75$ $\left.\mathrm{MHz}, \mathrm{CDCl}_{3}, \delta \mathrm{ppm}\right): 151.9,150.6,150.5,148.0,147.3,147.2,142.4,142.2,142.1,142.0,141.3$, $141.1,141.0,140.8,140.4,139.6,138.3,137.9,135.2$, 132.2, 132.0, 131.6, 131.4, 130.3, 130.2, $128.7,128.6,128.4,128.0,127.5,127.4,127.3,126.8,126.7,126.6,126.5,126.2,126.1,125.8$, $125.6,124.2,123.8,123.4,123.3,120.8,119.5,119.4,119.2,91.6,91.4,84.0,50.8,50.6,35.0$, 34.6, 31.6, 31.4, 24.9. HRMS (APCI, $m / z)$ : $\left[(\mathrm{M}+\mathrm{H})^{+}\right]$calcd for $\mathrm{C}_{142} \mathrm{H}_{139} \mathrm{BO}_{5}, 1935.0714$; found, 1935.0723.

\section{Synthesis of compound 16.}




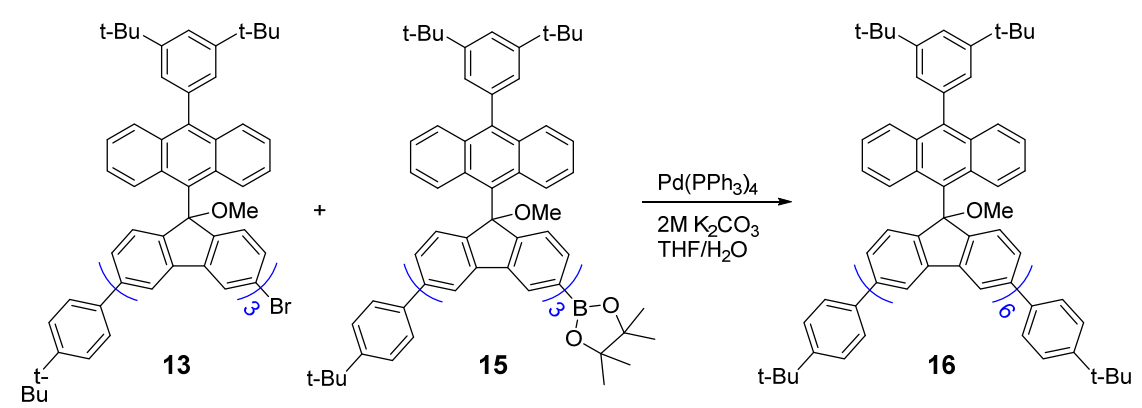

The synthesis is very similar to that for $\mathbf{8}$. Yellow solid $\mathbf{1 6}$ was obtained in $65 \%$ yield $(140 \mathrm{mg})$. ${ }^{1} \mathrm{H}$ NMR (300 MHz, $\mathrm{CDCl}_{3}, \delta$ ppm): 9.68-9.64 (m, 6H), 8.30-8.26 (m, 10H), 8.17 (s, 2H), 7.77$7.73(\mathrm{~m}, 6 \mathrm{H}), 7.65-7.26(\mathrm{~m}, 68 \mathrm{H}), 7.23-7.15(\mathrm{~m}, 6 \mathrm{H}), 7.05-6.99(\mathrm{~m}, 6 \mathrm{H}), 6.82-6.74(\mathrm{~m}, 6 \mathrm{H})$, 3.07-3.05 (m, 18H), 1.40-1.33 (m, 126H). $\left.{ }^{13} \mathrm{C} \mathrm{NMR} \mathrm{(75} \mathrm{MHz,} \mathrm{CDCl}_{3}, \delta \mathrm{ppm}\right): 150.6,150.5$, $148.0,147.3,142.3,142.2,142.0,141.1,141.0,140.7,140.4,140.4,138.3,138.3,137.9,132.0$, $131.9,131.6,131.4,130.2,128.6,128.3,128.3,128.0,127.7,127.6,127.5,127.4,126.8,126.6$, $126.1,125.8,125.5,124.2,123.8,123.4,123.4,123.3,120.8,119.5,119.4,119.1,91.3,50.8$, 35.0, 34.5, 31.6, 31.3. HRMS (APCI, $m / z):\left[(\mathrm{M}+\mathrm{H})^{+}\right]$calcd for $\mathrm{C}_{272} \mathrm{H}_{255} \mathrm{O}_{6}, 3616.9643$; found, 3616.9625 .

\section{Synthesis of radical FR-6.}
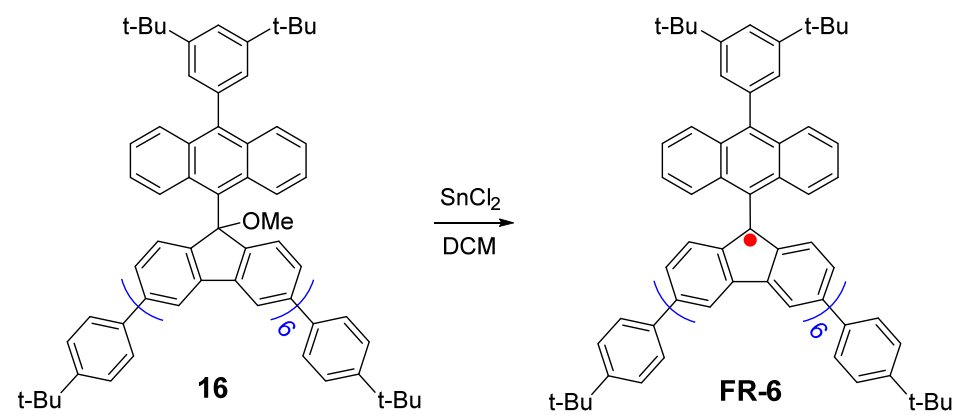

Radical FR-6 was synthesized similarly as described for synthesis of radical FR-1, black solid, yield 83\% (60 mg). MALDI-TOF Mass: $m / z$ 3428.8, calc. 3429.8. HRMS (ESI, $m / z, z=3$ ): [M] calcd for $\mathrm{C}_{266} \mathrm{H}_{236}, 1143.2817$; found, 1143.2826. 


\section{Theoretical calculations}

All results presented in this report have been obtained for a molecular model where the tert-butyl substituents are replaced by hydrogen atoms. Molecular geometries have been obtained at the UB3LYP/6-31G(d,p) level with Gaussian 09 package $^{1}$ utilizing a high performance computing cluster facility of NUS. From FR-2 onward, cis- and trans- isomers could exist and our calculations show that the energy difference between different isomers are substantially small (Figure S1) and thus only all-trans isomers are calculated for higher oligomers (FR-4 -FR-6). The multiple-radical characters $\left(y_{0}, y_{1}\right.$ and $\left.y_{2}\right)$ were first computed at UCAM-B3LYP/6-31G(d,p) using Yamaguchi's scheme:

$$
y_{i}=1-\frac{2 T_{i}}{1+T_{i}^{2}} ; \quad T_{i}=\frac{n_{H O N O-i}-n_{L U N O+i}}{2}
$$

on the basis of occupation numbers of the unoccupied natural orbitals LUNO, LUNO+1 and $\mathrm{LUNO}+2$, respectively (Table S1). ${ }^{2}$ However, the spin-unrestricted DFT calculations overestimated the relative energy levels of higher energy states and thus we also computed the radical nature of the electronic ground state and transition energies to higher states using the restricted active space spin flip method (RAS-SF) with Q-Chem 4.3 package. ${ }^{3}$ The radical character degree of the ground state singlet or doublet was estimated by the number of unpaired electrons $\left(N_{\mathrm{U}}\right)$ according to equation 2 , where $\left\{n_{\mathrm{i}}\right\}$ are the natural occupation numbers from the one-particle density matrix. ${ }^{4}$

$$
N_{U}=\sum_{i}\left(1-a b s\left(1-n_{i}\right)\right)
$$

Since the calculated occupation numbers of HONO-i and LUNO+i $(i=0,1,2)$ also meet the basis of Yamguchi's scheme: $n_{\mathrm{HONO}-\mathrm{i}}+n_{\mathrm{LUNO}+\mathrm{i}}=2$ (Figure S2 and Figure 4 in main manuscript), the multiple radical characters $y_{\mathrm{i}}$ were also calculated by using Yamaguchi's scheme for comparison. 

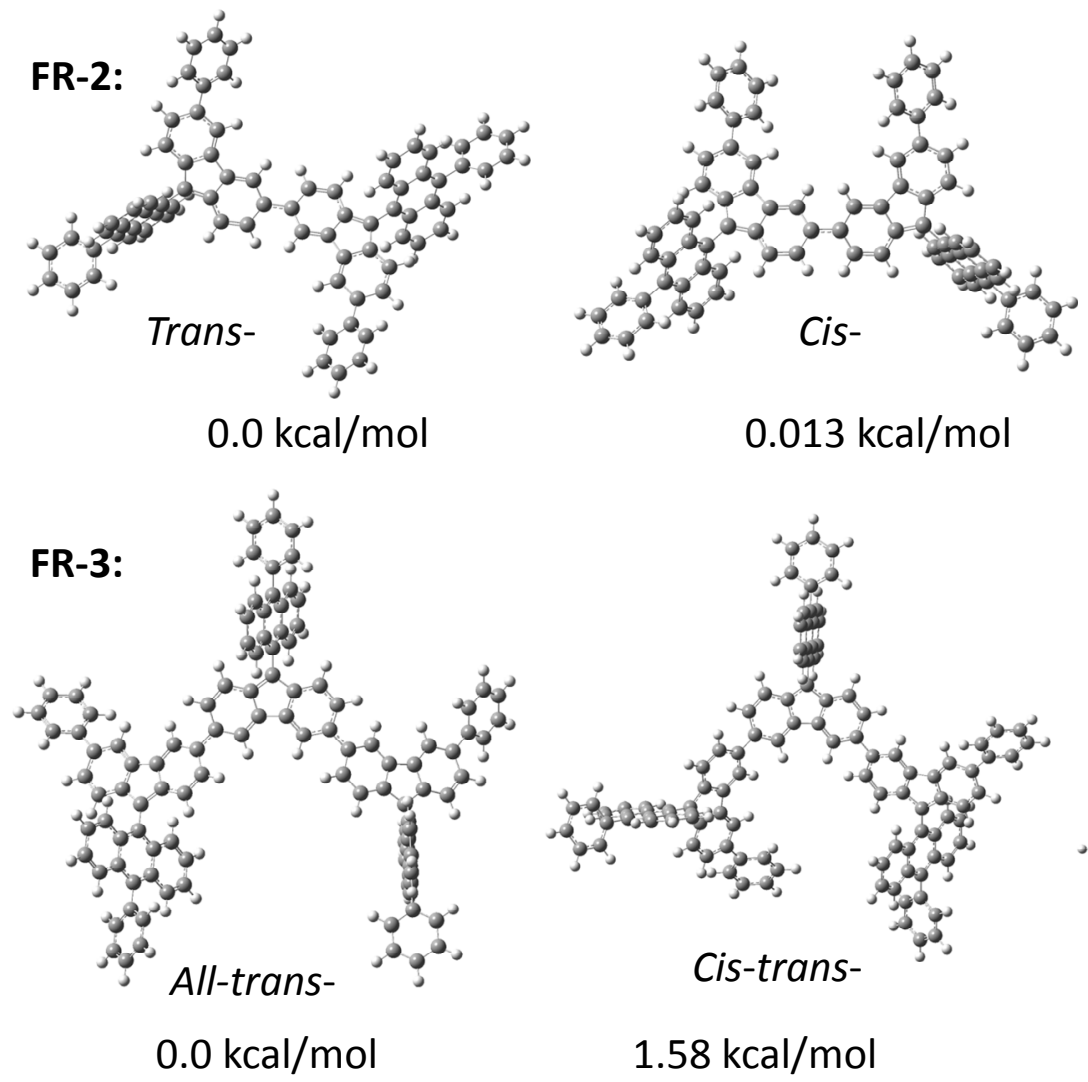

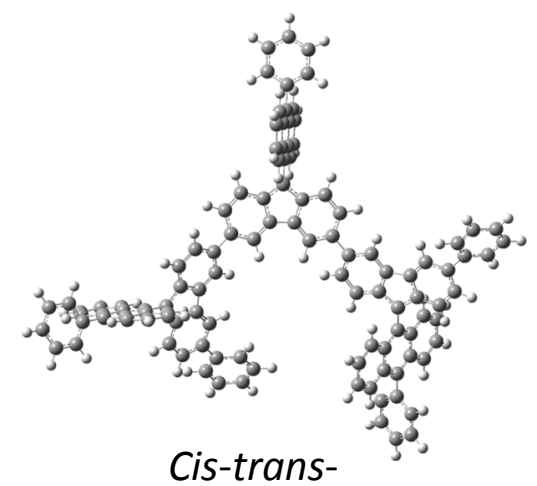

$1.58 \mathrm{kcal} / \mathrm{mol}$

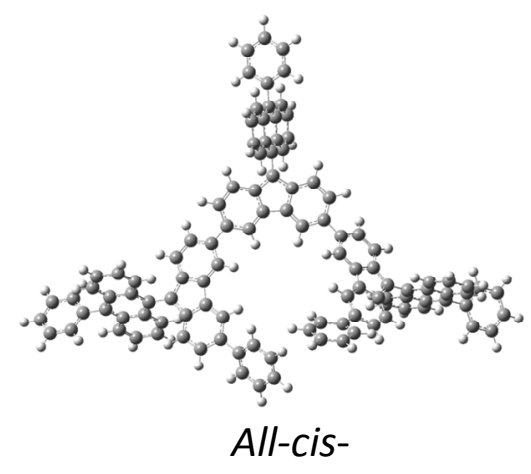

$2.50 \mathrm{kcal} / \mathrm{mol}$

Figure S1. Optimised geometries (UB3LYP/6-31G(d,p)) and relative energies of different cis/trans- isomers of FR-2 and FR-3.

Table S1. Calculated (UCAM-B3LYP/6-31G(d,p)) polyradical characters for FR-2-FR-6.

\begin{tabular}{llll}
\hline Molecules & $y_{0}$ & $y_{1}$ & $y_{2}$ \\
\hline FR-2 & 0.62 & 0.0 & 0.0 \\
FR-3 & 0.50 & 0.0 & 0.0 \\
FR-4 & 0.75 & 0.45 & 0.0 \\
FR-5 & 0.63 & 0.43 & 0.0 \\
FR-6 & 0.83 & 0.56 & 0.41 \\
\hline
\end{tabular}


(b)
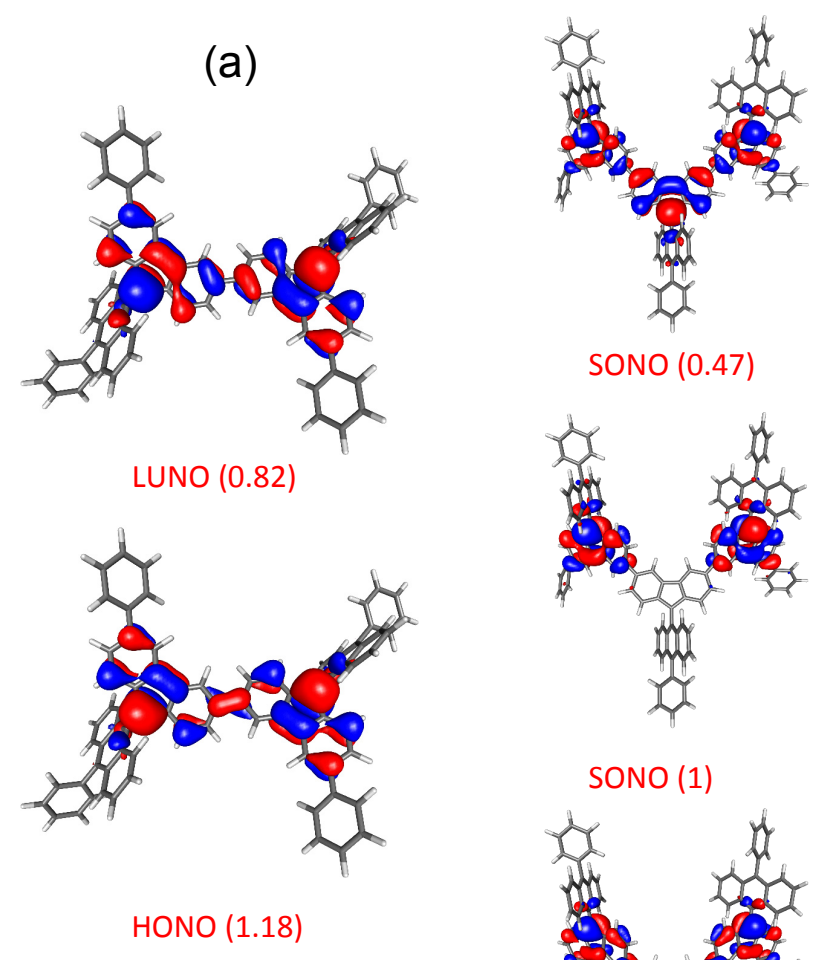

SONO (1)

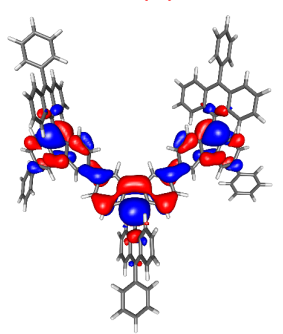

SONO (1.53) 
(c)

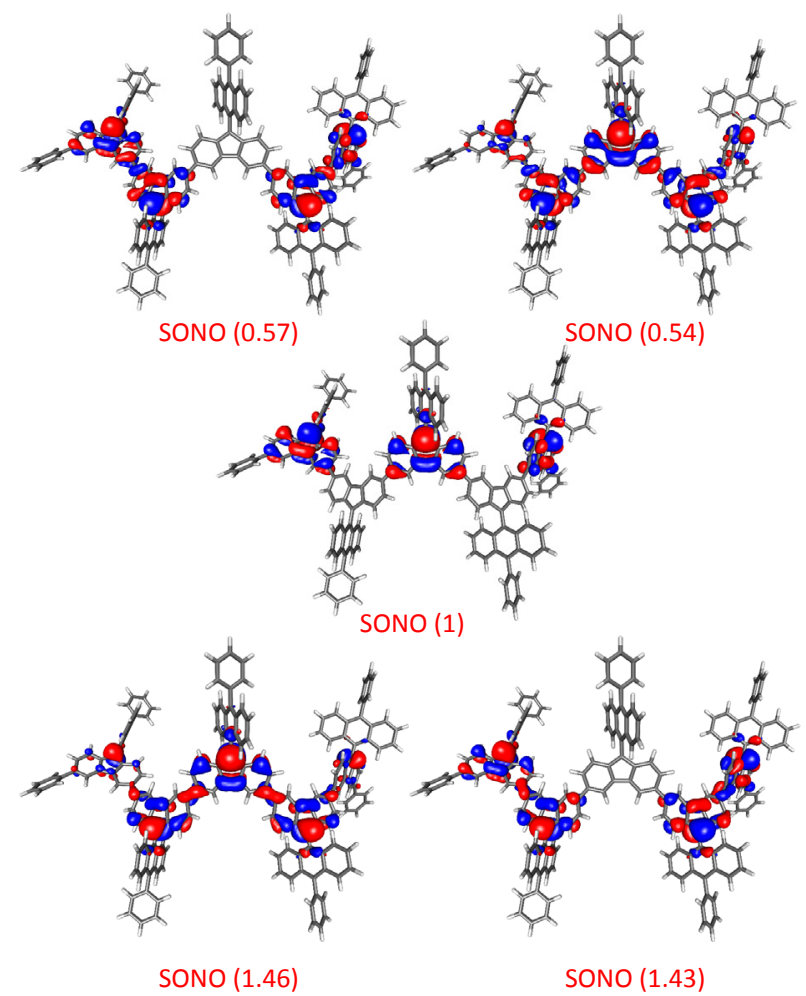

(d)
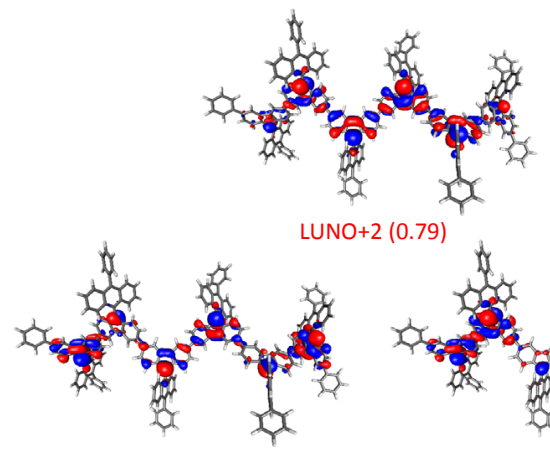

LUNO (0.90)

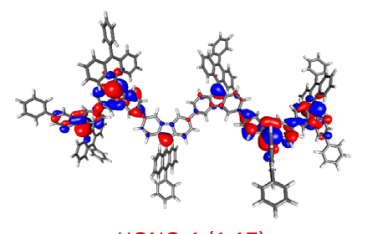

HONO-1 (1.17)

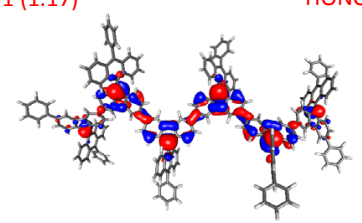

HONO-2 (1.21)

Figure S2. Natural orbital diagrams and occupancy numbers for (a) FR-2, (b) FR-3, (c) FR-5 and (d) FR-6 in all-trans forms.

Table S2. Calculated (RAS-SF) vertical energy gaps (in $\mathrm{kcal} / \mathrm{mol}$ ) from low spin ground state to high spin excited states for FR-2-FR-6.

\begin{tabular}{|c|c|c|c|c|c|}
\hline Molecule & FR2 & FR4 & FR6 & FR3 & FR5 \\
\hline triplet & 0.97 & 0.643 & 0.352 & & \\
\hline quintet & & 2.324 & 1.332 & & \\
\hline septet & & & 2.654 & & \\
\hline doublet & & & & 0.991 & 0.599 \\
\hline quartet & & & & 1.498 & 0.945 \\
\hline sextet & & & & & 2.444 \\
\hline
\end{tabular}


3. VT NMR spectra

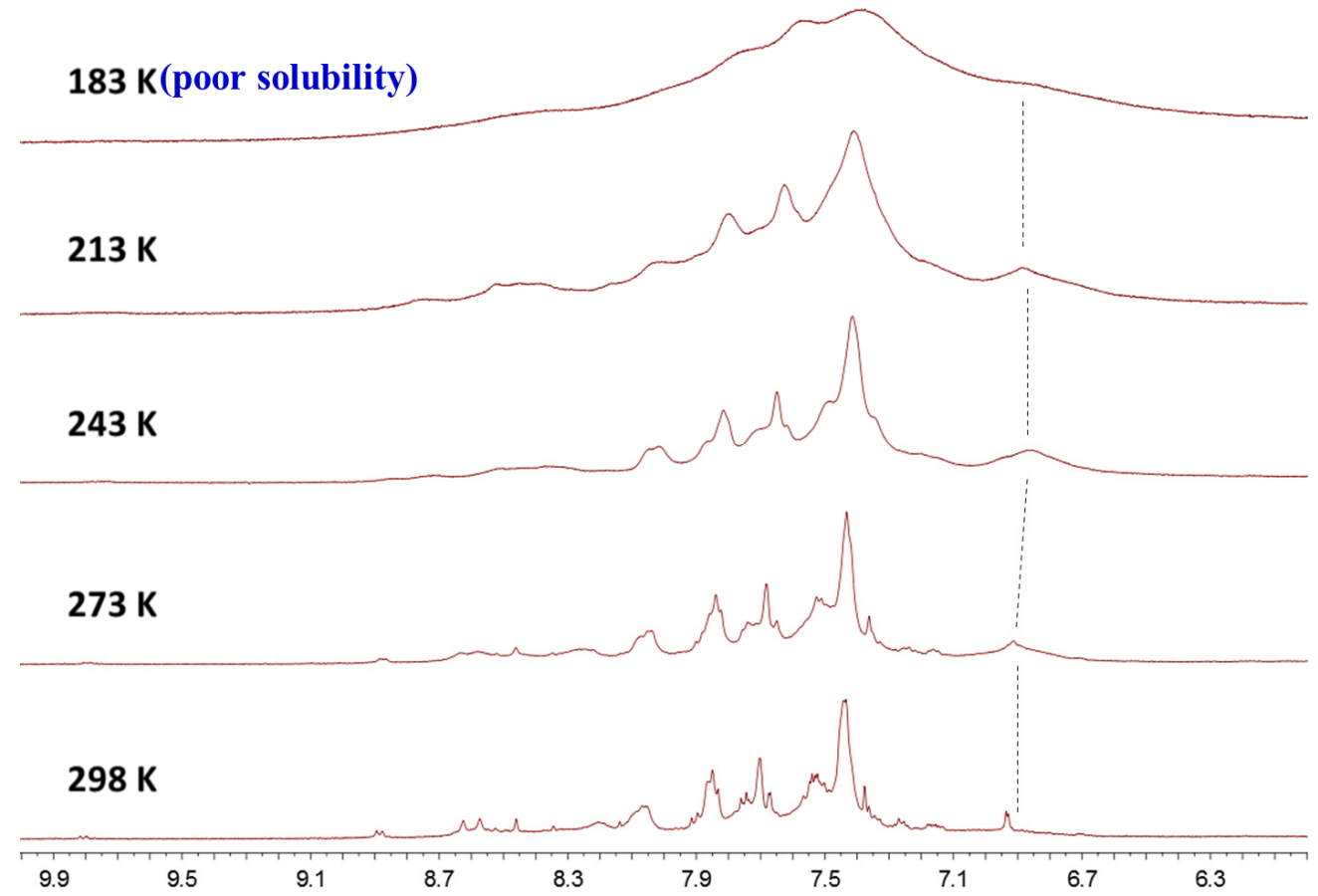

Figure S3. VT ${ }^{1} \mathrm{H}$ NMR spectra of FR-3 in $\mathrm{CD}_{2} \mathrm{Cl}_{2}(500 \mathrm{MHz})$.

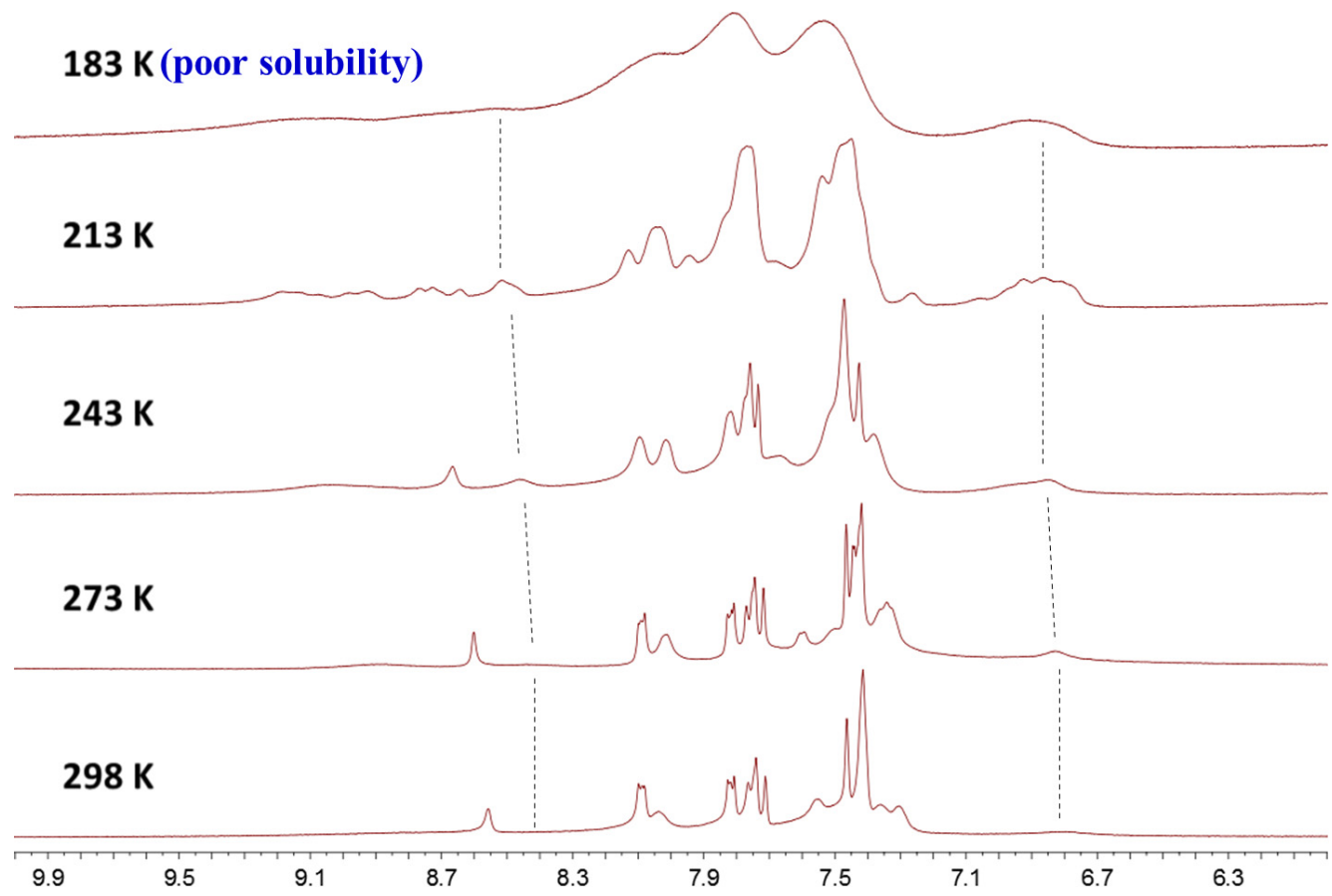

Figure S4. VT ${ }^{1}$ H NMR spectra of FR-4 in $d_{8}$-THF (500 MHz). 


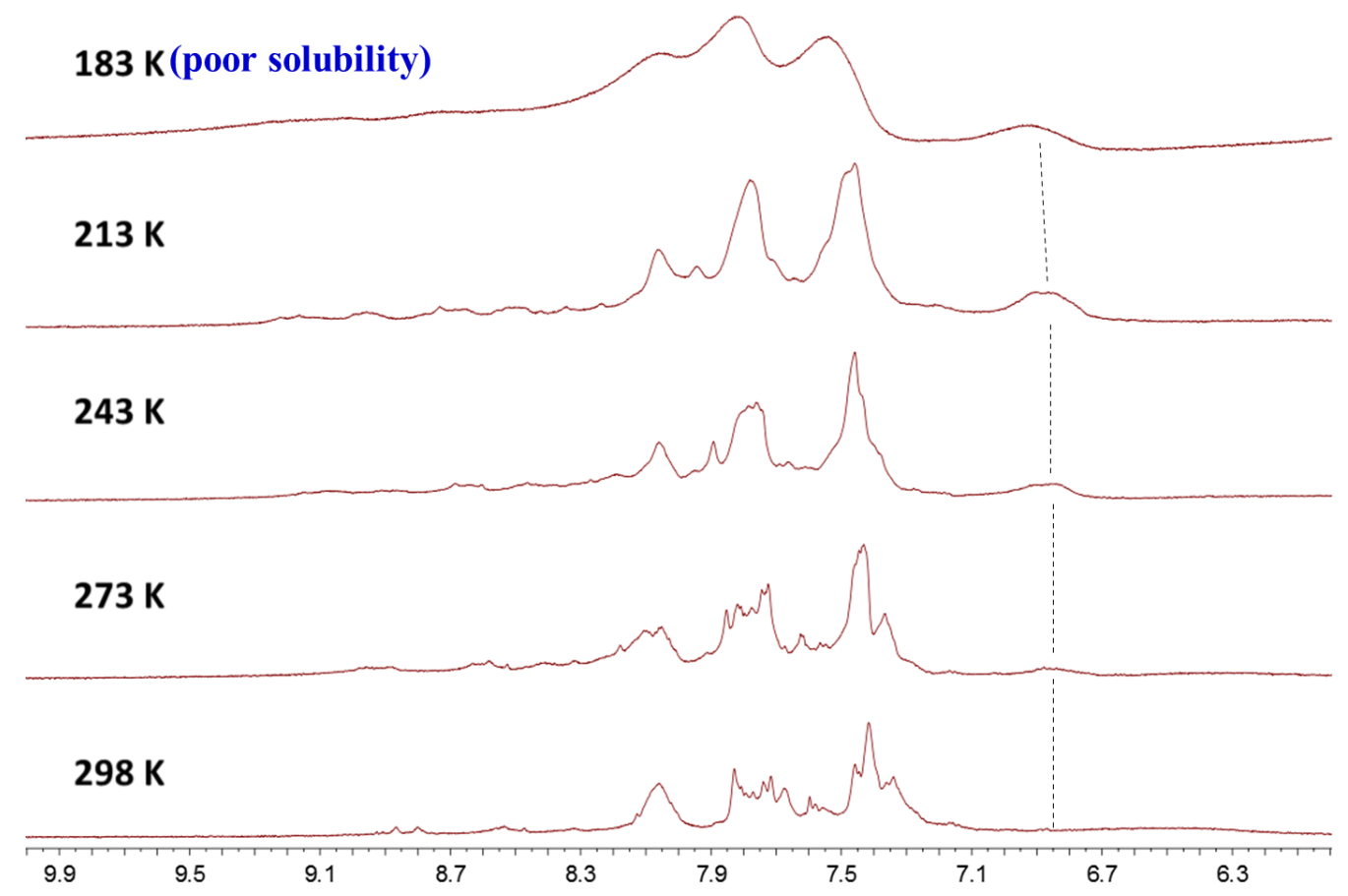

Figure S5. VT ${ }^{1}$ H NMR spectra of FR-5 in $d_{8}$-THF (500 MHz).

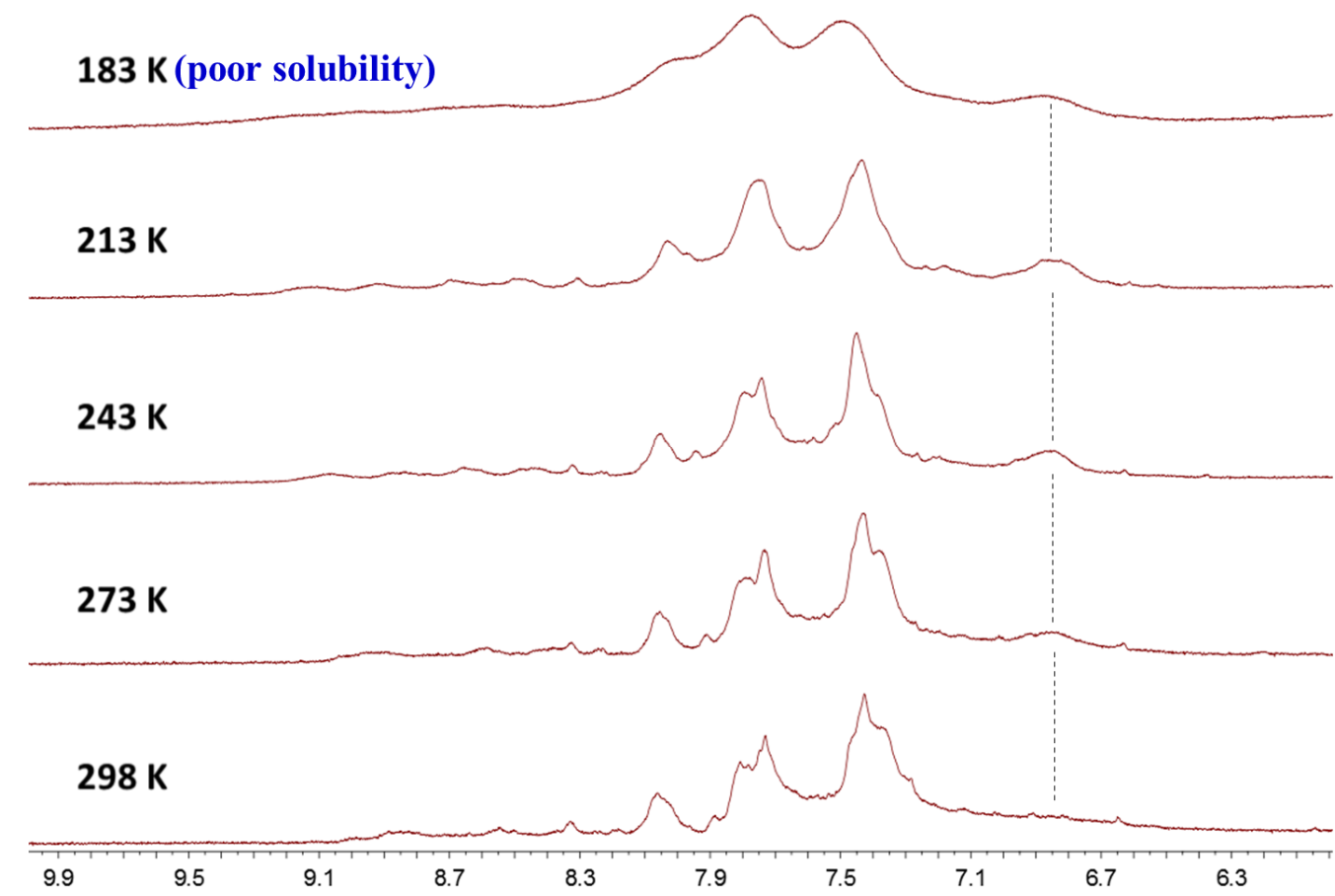

Figure S6. VT ${ }^{1}$ H NMR spectra of FR-6 in $d_{8}$-THF (500 MHz). 


\section{ESR spectra and UV-vis-NIR spectra}
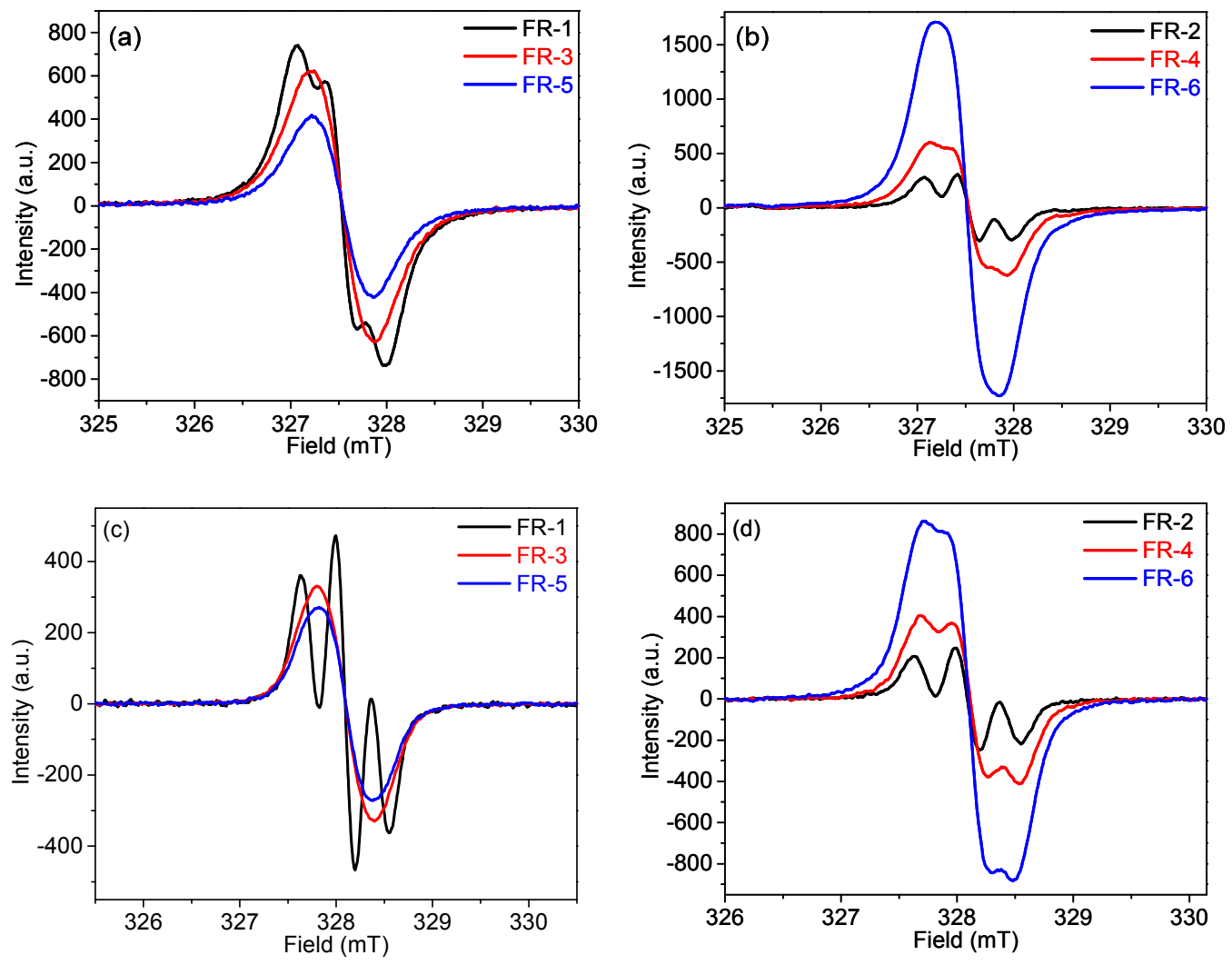

Figure S7. ESR spectra recorded in toluene: (a) FR-1, FR-3 and FR-5 (0.09 mM, $298 \mathrm{~K})$; (b) FR-2, FR-4 and FR-6 (0.9 mM, $298 \mathrm{~K})$; (c) FR-1, FR-3 and FR-5 (0.09 mM, $183 \mathrm{~K})$; (d) FR-2, FR-4 and FR-6 (0.9 mM, $183 \mathrm{~K})$

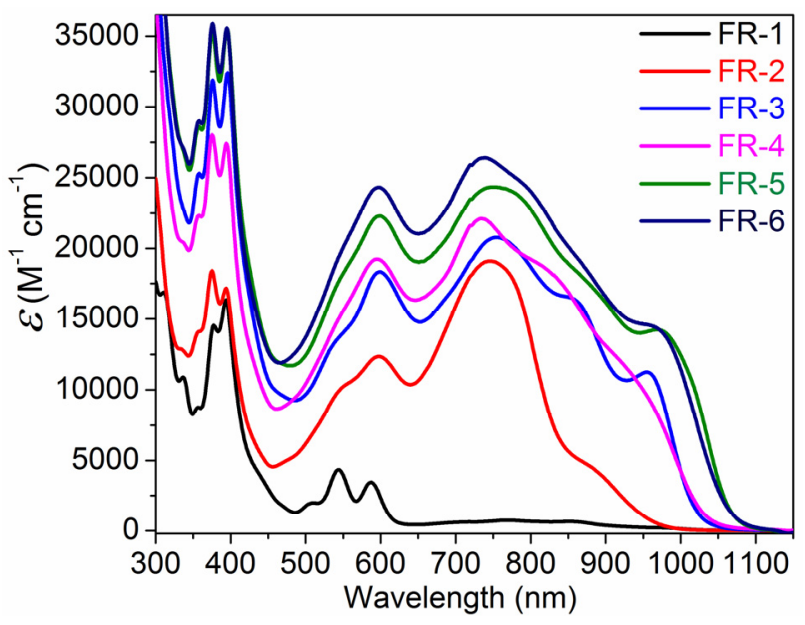

Figure S8. UV-vis-NIR spectra of FR-n $(n=1-6)$ in toluene. 


\section{Z-Scan curves}
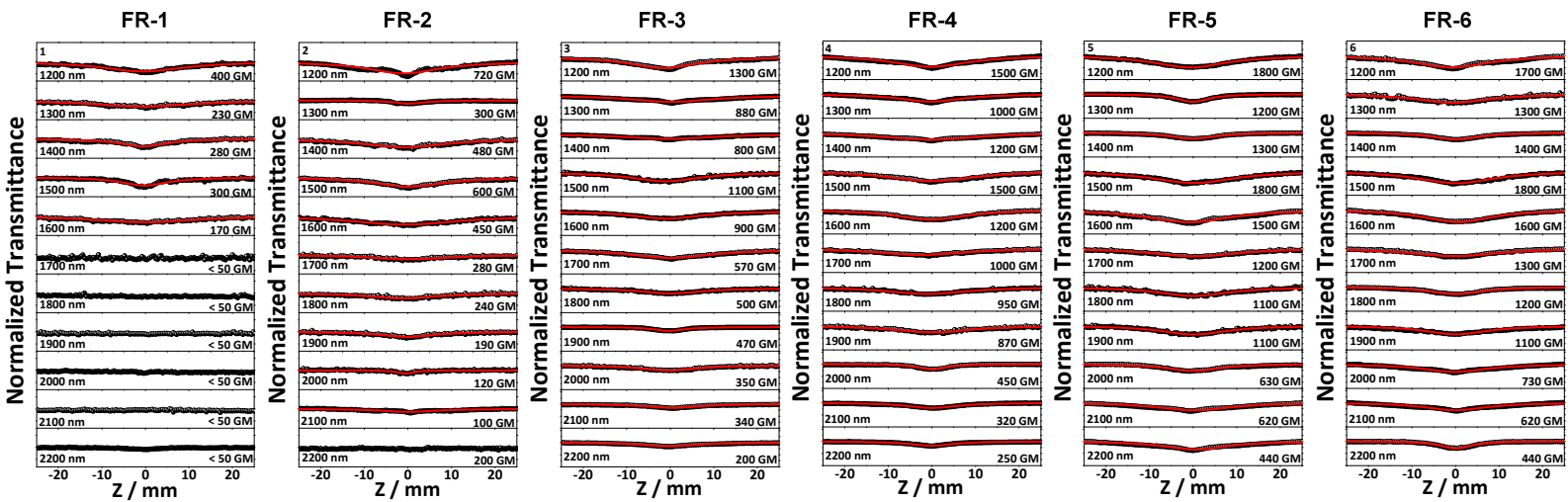

Figure S9. Z-scan curves of FR-n ( $n=1-6)$ measured in toluene by photoexcitation in the range from 1200 to $2200 \mathrm{~nm}$.

\section{Transient absorption spectra}
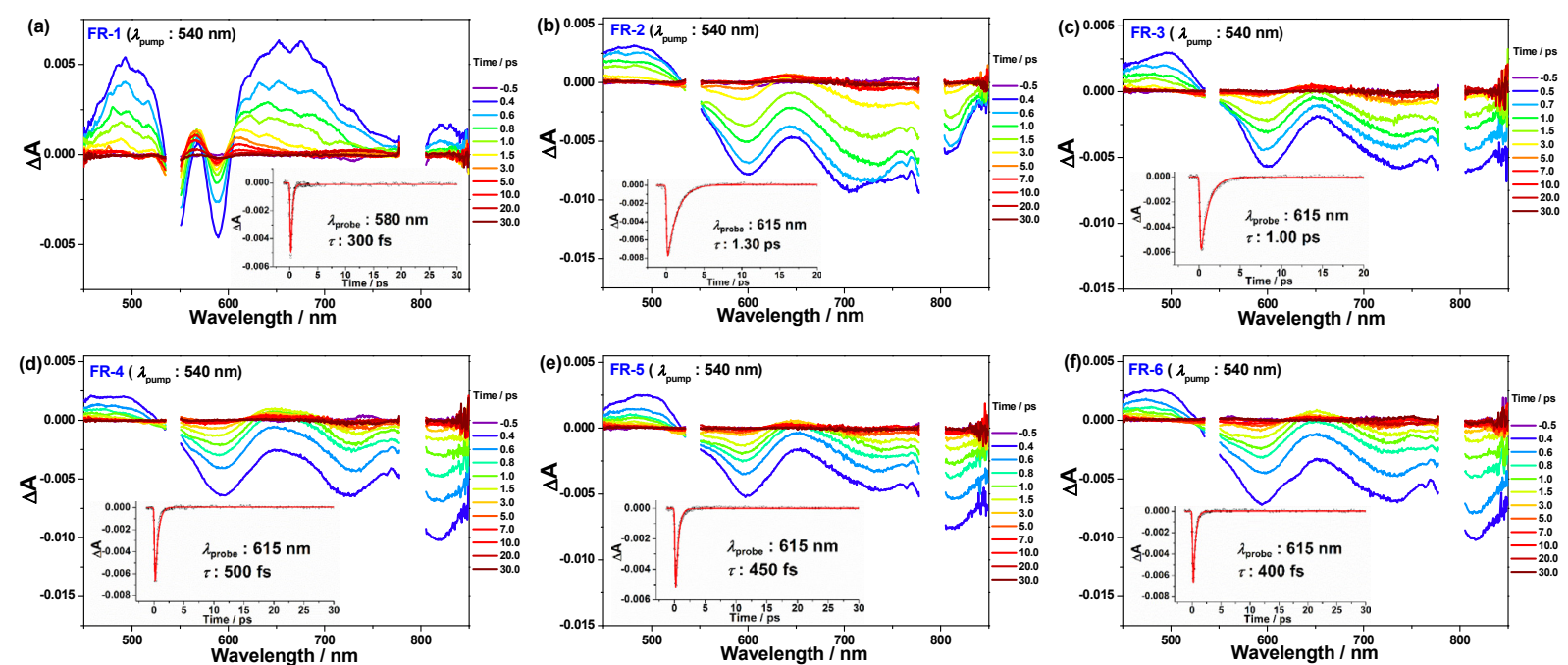

Figure S10. Transient absorption spectra of (a) FR-1, (b) FR-2, (c) FR-3, (d) FR-4, (e) FR-5, and (f) FR-6 recorded in toluene. Insets are the decay profiles. 


\section{Chemical reduction}
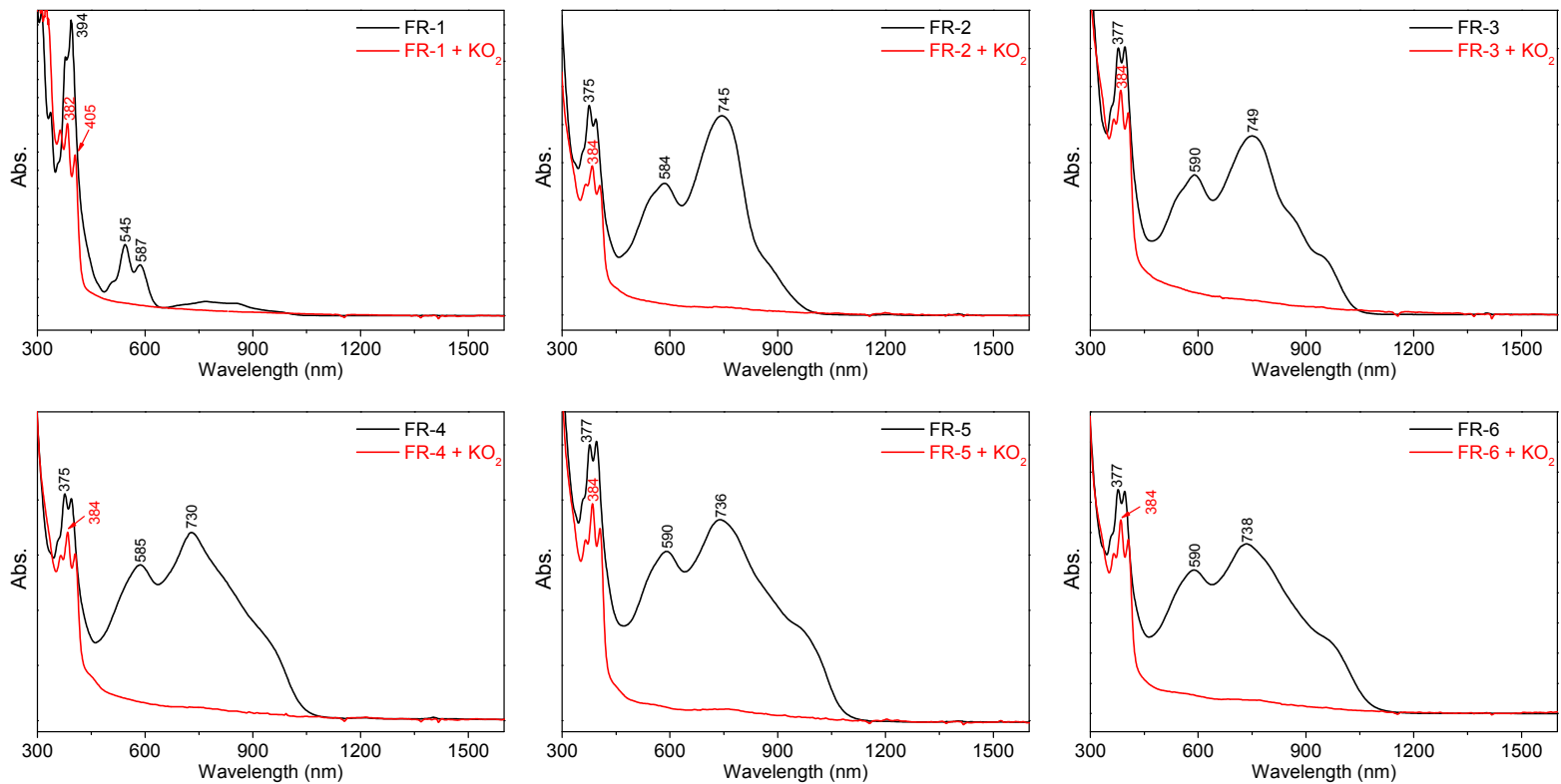

Figure S11. UV-vis-NIR absorption spectra of neutral FR-n $(n=1-6)$ and their fully reduced anions by excessive $\mathrm{KO}_{2}$ (5 equiv) with 18-crown-6 (1:1) in DCM.
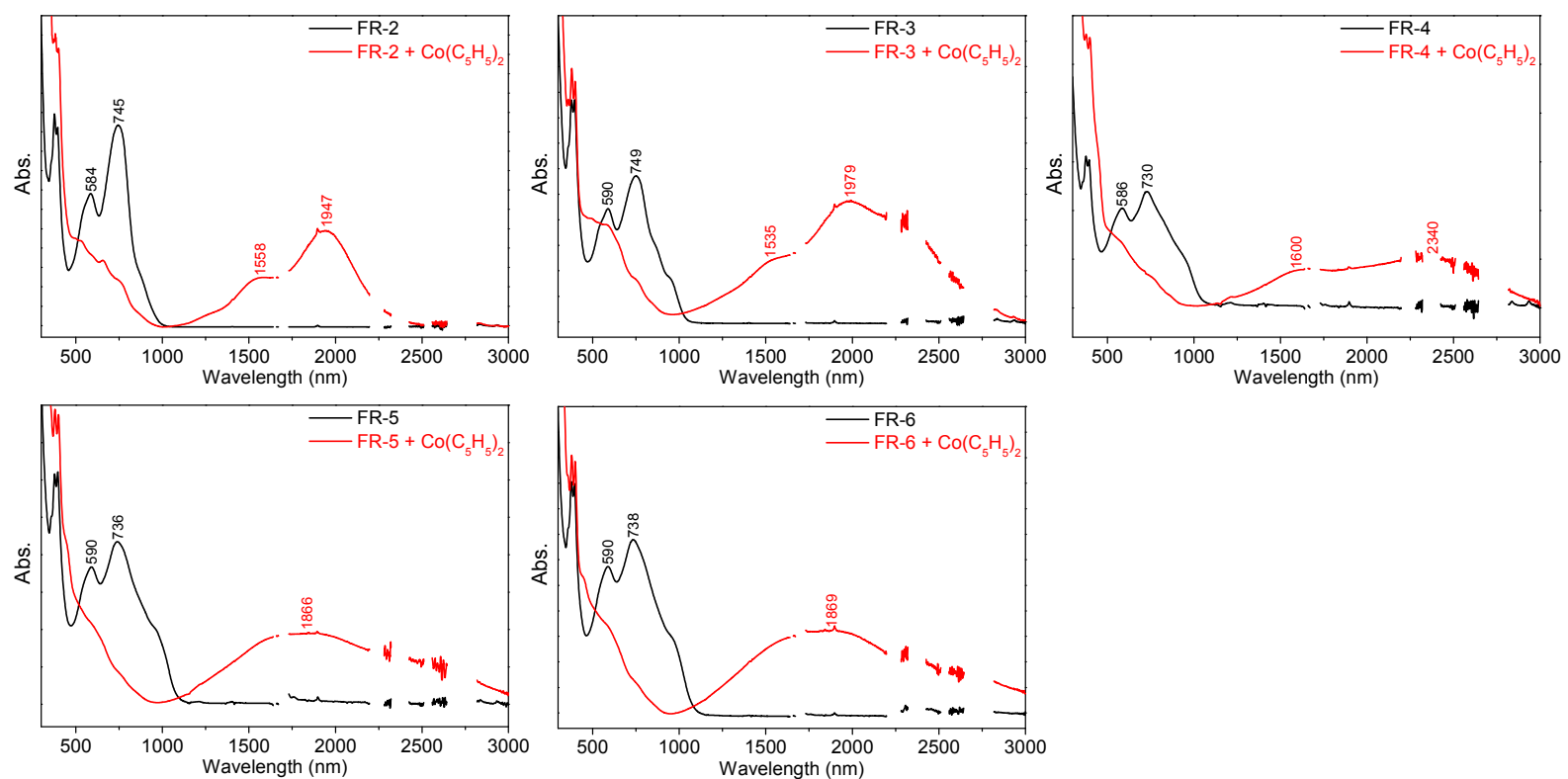

Figure S12. UV-vis-NIR absorption spectra of neutral FR-n $(n=2-6)$ and their reduced (poly)radical mono-anions by excessive $\mathrm{CoCp}_{2}(1.5$ equiv) in DCM. 


\section{X-ray crystallographic data for FR-1 and FR-2.}

8.1 X-ray crystallographic data for FR-1.

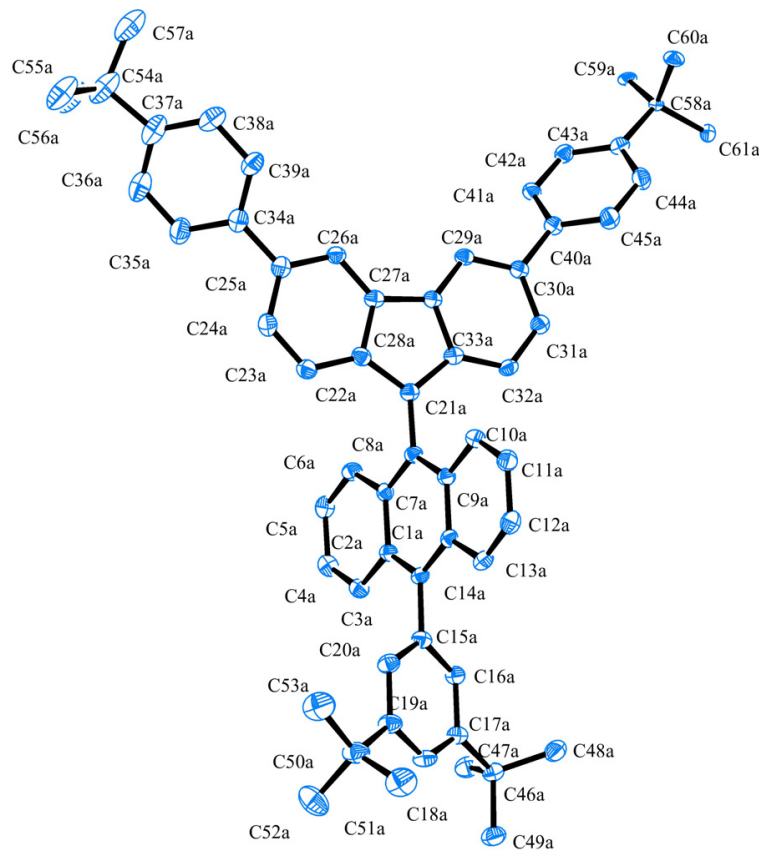

Molecule's view

Table S3. Crystal data and structure refinement for FR-1.

$\begin{array}{lll}\text { Empirical formula } & \mathrm{C}_{91.5} \mathrm{H}_{91.5} & \\ \text { Formula weight } & 1191.14 & \\ \text { Temperature } & 100(2) \mathrm{K} & \\ \text { Wavelength } & 1.54178 \AA & \\ \text { Crystal system } & \text { Monoclinic } & \\ \text { Space group } & \mathrm{C} 2 / \mathrm{c} & \alpha=90^{\circ} \\ \text { Unit cell dimensions } & \mathrm{a}=47.8736(15) \AA & \beta=97.306(2)^{\circ} \\ & \mathrm{b}=19.1675(6) \AA & \gamma=90^{\circ} \\ & \mathrm{c}=16.5421(5) \AA & \\ \text { Volume } & 15056.1(8) \AA \AA^{3} & \\ Z & 8 & \\ \text { Density (calculated) } & 1.051 \mathrm{Mg}^{3} & \\ \text { Absorption coefficient } & 0.440 \mathrm{~mm}^{-1} & \end{array}$


$\mathrm{F}(000)$

Crystal size

Theta range for data collection

Index ranges

Reflections collected

Independent reflections

Completeness to theta $=67.679^{\circ}$

Refinement method

Data / restraints / parameters

Goodness-of-fit on $\mathrm{F}^{2}$

Final R indices [I $>2 \operatorname{sigma}(\mathrm{I})]$

$\mathrm{R}$ indices (all data)

Extinction coefficient

Largest diff. peak and hole
5124

$0.547 \times 0.286 \times 0.146 \mathrm{~mm}^{3}$

2.486 to $65.229^{\circ}$

$-55<=\mathrm{h}<=56,-22<=\mathrm{k}<=15,-19<=\mathrm{l}<=19$

42041

$12573[\mathrm{R}(\mathrm{int})=0.0636]$

$92.3 \%$

Full-matrix least-squares on $\mathrm{F}^{2}$

12573 / 425 / 938

1.084

$\mathrm{R} 1=0.0998, \mathrm{wR} 2=0.2720$

$\mathrm{R} 1=0.1224, \mathrm{wR} 2=0.2901$

$0.000005(17)$

0.959 and -0.703 e. $\AA^{-3}$ 
Table S4. Atomic coordinates ( $x \mathbf{1 0}^{4}$ ) and equivalent isotropic displacement parameters $\left(\AA^{2} \times 1^{3}\right)$ for FR-1. $U(e q)$ is defined as one third of the trace of the orthogonalized $U^{i j}$ tensor.

\begin{tabular}{|c|c|c|c|c|}
\hline & $\mathrm{x}$ & $\mathrm{y}$ & $\mathrm{z}$ & $\mathrm{U}(\mathrm{eq})$ \\
\hline$C(1 \mathrm{~A})$ & $3174(1)$ & $6865(2)$ & $2416(2)$ & $21(1)$ \\
\hline$C(2 A)$ & $3339(1)$ & $6290(2)$ & $2728(2)$ & $23(1)$ \\
\hline$C(3 \mathrm{~A})$ & $3513(1)$ & $5917(2)$ & $2240(2)$ & $27(1)$ \\
\hline $\mathrm{C}(4 \mathrm{~A})$ & $3681(1)$ & $5384(2)$ & $2564(2)$ & $30(1)$ \\
\hline$C(5 \mathrm{~A})$ & $3681(1)$ & $5181(2)$ & $3389(2)$ & $30(1)$ \\
\hline$C(6 A)$ & $3515(1)$ & $5518(2)$ & $3870(2)$ & $27(1)$ \\
\hline$C(7 A)$ & $3338(1)$ & $6087(2)$ & $3565(2)$ & $23(1)$ \\
\hline $\mathrm{C}(8 \mathrm{~A})$ & $3169(1)$ & $6448(2)$ & $4063(2)$ & $23(1)$ \\
\hline $\mathrm{C}(9 \mathrm{~A})$ & $3002(1)$ & $7013(2)$ & $3746(2)$ & $22(1)$ \\
\hline $\mathrm{C}(10 \mathrm{~A})$ & $2835(1)$ & $7410(2)$ & $4243(2)$ & $26(1)$ \\
\hline $\mathrm{C}(11 \mathrm{~A})$ & $2682(1)$ & $7970(2)$ & $3939(2)$ & $30(1)$ \\
\hline $\mathrm{C}(12 \mathrm{~A})$ & $2685(1)$ & $8171(2)$ & $3125(2)$ & $30(1)$ \\
\hline$C(13 A)$ & $2836(1)$ & $7815(2)$ & $2622(2)$ & $27(1)$ \\
\hline $\mathrm{C}(14 \mathrm{~A})$ & $3007(1)$ & $7226(2)$ & $2916(2)$ & $22(1)$ \\
\hline$C(15 A)$ & $3183(1)$ & $7107(2)$ & $1562(2)$ & $25(1)$ \\
\hline$C(16 A)$ & $3045(1)$ & $6758(2)$ & $897(2)$ & $25(1)$ \\
\hline $\mathrm{C}(17 \mathrm{~A})$ & $3051(1)$ & $7004(2)$ & $101(2)$ & $26(1)$ \\
\hline $\mathrm{C}(18 \mathrm{~A})$ & $3199(1)$ & $7618(2)$ & $7(2)$ & $30(1)$ \\
\hline $\mathrm{C}(19 \mathrm{~A})$ & $3339(1)$ & $7984(2)$ & $666(2)$ & $31(1)$ \\
\hline$C(20 A)$ & $3331(1)$ & $7715(2)$ & $1442(2)$ & $28(1)$ \\
\hline$C(21 \mathrm{~A})$ & $3169(1)$ & $6249(2)$ & $4925(2)$ & $23(1)$ \\
\hline $\mathrm{C}(22 \mathrm{~A})$ & $3387(1)$ & $6395(2)$ & $5578(2)$ & $26(1)$ \\
\hline$C(23 \mathrm{~A})$ & $3646(1)$ & $6741(2)$ & $5592(2)$ & $29(1)$ \\
\hline$C(24 \mathrm{~A})$ & $3815(1)$ & $6820(2)$ & $6326(2)$ & $31(1)$ \\
\hline$C(25 \mathrm{~A})$ & $3736(1)$ & $6564(2)$ & $7063(2)$ & $28(1)$ \\
\hline$C(26 A)$ & $3476(1)$ & $6223(2)$ & $7047(2)$ & $25(1)$ \\
\hline $\mathrm{C}(27 \mathrm{~A})$ & $3309(1)$ & $6126(2)$ & $6319(2)$ & $24(1)$ \\
\hline $\mathrm{C}(28 \mathrm{~A})$ & $3033(1)$ & $5791(2)$ & $6116(2)$ & $23(1)$ \\
\hline$C(29 A)$ & $2868(1)$ & $5416(2)$ & $6589(2)$ & $23(1)$ \\
\hline $\mathrm{C}(30 \mathrm{~A})$ & $2605(1)$ & $5160(2)$ & $6231(2)$ & $24(1)$ \\
\hline
\end{tabular}




\begin{tabular}{|c|c|c|c|c|}
\hline & $\mathrm{x}$ & $\mathrm{y}$ & z & $\mathrm{U}(\mathrm{eq})$ \\
\hline$C(31 \mathrm{~A})$ & $2520(1)$ & $5287(2)$ & $5400(2)$ & $26(1)$ \\
\hline$C(32 \mathrm{~A})$ & $2687(1)$ & $5638(2)$ & $4919(2)$ & $25(1)$ \\
\hline$C(33 A)$ & $2948(1)$ & $5895(2)$ & $5271(2)$ & $23(1)$ \\
\hline$C(34 \mathrm{~A})$ & 3923(1) & $6628(2)$ & $7835(2)$ & $31(1)$ \\
\hline$C(35 \mathrm{~A})$ & $4215(1)$ & $6553(2)$ & $7855(3)$ & $42(1)$ \\
\hline$C(36 A)$ & $4392(1)$ & $6551(2)$ & $8583(3)$ & $51(1)$ \\
\hline$C(37 A)$ & 4293(1) & $6633(2)$ & $9320(3)$ & $49(1)$ \\
\hline$C(38 A)$ & $4002(1)$ & $6730(2)$ & $9305(3)$ & $45(1)$ \\
\hline$C(39 A)$ & $3825(1)$ & $6731(2)$ & $8574(2)$ & $37(1)$ \\
\hline$C(40 A)$ & $2422(1)$ & $4765(2)$ & $6725(2)$ & $25(1)$ \\
\hline$C(41 A)$ & $2527(1)$ & $4223(2)$ & $7244(2)$ & $27(1)$ \\
\hline$C(42 A)$ & $2352(1)$ & $3836(2)$ & $7672(2)$ & $28(1)$ \\
\hline$C(43 A)$ & $2065(1)$ & $3980(2)$ & $7627(2)$ & $26(1)$ \\
\hline$C(44 A)$ & 1964(1) & $4537(2)$ & $7121(2)$ & $33(1)$ \\
\hline$C(45 \mathrm{~A})$ & $2137(1)$ & $4914(2)$ & $6677(2)$ & $33(1)$ \\
\hline$C(46 A)$ & 2901(1) & $6592(2)$ & $-618(2)$ & $29(1)$ \\
\hline $\mathrm{C}(47 \mathrm{~A})$ & $3047(1)$ & $5875(2)$ & $-648(2)$ & $37(1)$ \\
\hline$C(48 A)$ & $2589(1)$ & $6489(2)$ & $-510(2)$ & $37(1)$ \\
\hline$C(49 A)$ & $2916(1)$ & $6952(2)$ & $-1436(2)$ & $37(1)$ \\
\hline$C(50 A)$ & $3486(1)$ & $8675(2)$ & $517(2)$ & $39(1)$ \\
\hline$C(51 A)$ & $3266(1)$ & $9190(2)$ & $123(3)$ & $51(1)$ \\
\hline$C(52 A)$ & $3711(1)$ & $8550(2)$ & $-56(3)$ & $55(1)$ \\
\hline$C(53 A)$ & $3627(1)$ & $9003(2)$ & $1314(2)$ & $57(1)$ \\
\hline$C(54 A)$ & $4517(2)$ & $6638(4)$ & $10046(5)$ & $69(1)$ \\
\hline $\mathrm{C}(55 \mathrm{~A})$ & $4680(2)$ & $7324(5)$ & $10095(6)$ & $73(2)$ \\
\hline$C(56 A)$ & $4724(2)$ & $6023(5)$ & $10010(9)$ & $68(2)$ \\
\hline$C(57 A)$ & $4370(2)$ & $6570(6)$ & $10818(6)$ & $73(2)$ \\
\hline$C\left(54^{\prime}\right)$ & $4465(2)$ & $6589(4)$ & $10197(5)$ & $69(1)$ \\
\hline$C\left(55^{\prime}\right)$ & $4519(2)$ & $7325(4)$ & $10534(7)$ & $78(2)$ \\
\hline$C\left(56^{\prime}\right)$ & $4751(2)$ & $6234(6)$ & $10132(8)$ & $69(2)$ \\
\hline$C\left(57^{\prime}\right)$ & $4313(2)$ & $6178(5)$ & $10815(6)$ & $70(2)$ \\
\hline $\mathrm{C}(58 \mathrm{~A})$ & $1864(2)$ & $3581(4)$ & $8096(4)$ & $15(2)$ \\
\hline
\end{tabular}




\begin{tabular}{|c|c|c|c|c|}
\hline C(59A) & $2005(3)$ & $2950(5)$ & $8537(6)$ & $22(2)$ \\
\hline$C(60 A)$ & $1756(2)$ & 4079(5) & $8722(6)$ & $28(2)$ \\
\hline$C(61 \mathrm{~A})$ & $1610(2)$ & $3316(5)$ & $7508(6)$ & $20(2)$ \\
\hline$C\left(58^{\prime}\right)$ & 1873(3) & $3569(7)$ & $8131(8)$ & $65(4)$ \\
\hline $\mathrm{C}\left(59^{\prime}\right)$ & 1993(4) & $2859(8)$ & $8413(11)$ & $67(4)$ \\
\hline$C\left(60^{\prime}\right)$ & 1837(3) & 4016(8) & $8887(9)$ & $72(4)$ \\
\hline \multirow[t]{2}{*}{$C\left(61^{\prime}\right)$} & 1582(3) & $3446(9)$ & 7634(9) & $72(4)$ \\
\hline & $\mathrm{x}$ & $\mathrm{y}$ & $\mathrm{z}$ & $\mathrm{U}(\mathrm{eq})$ \\
\hline $\mathrm{C}(1 \mathrm{~B})$ & 5000 & $4870(3)$ & 7500 & $31(1)$ \\
\hline $\mathrm{C}(2 \mathrm{~B})$ & $4794(1)$ & 4431(2) & $7790(2)$ & $30(1)$ \\
\hline$C(3 B)$ & $4546(1)$ & $4578(2)$ & $8124(2)$ & $36(1)$ \\
\hline$C(4 B)$ & $4384(1)$ & $4039(2)$ & $8347(2)$ & $37(1)$ \\
\hline$C(5 B)$ & $4459(1)$ & $3338(2)$ & $8247(2)$ & $33(1)$ \\
\hline $\mathrm{C}(6 \mathrm{~B})$ & $4707(1)$ & $3186(2)$ & 7913(2) & $31(1)$ \\
\hline$C(7 B)$ & $4869(1)$ & $3721(2)$ & $7685(2)$ & $28(1)$ \\
\hline $\mathrm{C}(8 \mathrm{~B})$ & $4277(1)$ & $2760(2)$ & $8477(2)$ & $33(1)$ \\
\hline $\mathrm{C}(9 \mathrm{~B})$ & $3985(1)$ & $2842(2)$ & $8415(2)$ & $35(1)$ \\
\hline$C(10 B)$ & $3817(1)$ & $2310(2)$ & $8641(2)$ & $33(1)$ \\
\hline$C(11 B)$ & $3928(1)$ & $1680(2)$ & $8937(2)$ & $33(1)$ \\
\hline$C(12 B)$ & $4219(1)$ & 1597(2) & $8987(3)$ & $39(1)$ \\
\hline$C(13 B)$ & $4389(1)$ & 2121(2) & $8754(2)$ & $40(1)$ \\
\hline$C(14 B)$ & 5000 & $5641(3)$ & 7500 & $35(1)$ \\
\hline$C(15 B)$ & $4829(1)$ & 5998(2) & $6876(3)$ & $48(1)$ \\
\hline$C(16 B)$ & $4833(1)$ & $6740(3)$ & $6869(4)$ & $63(2)$ \\
\hline$C(17 B)$ & $4656(1)$ & 7074(3) & 6188(4) & $77(2)$ \\
\hline$C(18 B)$ & $4496(1)$ & 6693(4) & $5613(4)$ & $79(2)$ \\
\hline$C(19 B)$ & 4491(1) & 5971(3) & $5635(3)$ & $68(2)$ \\
\hline$C(20 B)$ & $4654(1)$ & $5631(3)$ & $6247(3)$ & $56(1)$ \\
\hline \multirow[t]{2}{*}{$\mathrm{C}(21 \mathrm{~B})$} & 5000 & 7099(4) & 7500 & $73(3)$ \\
\hline & $\mathrm{x}$ & $\mathrm{y}$ & $\mathrm{z}$ & $\mathrm{U}(\mathrm{eq})$ \\
\hline
\end{tabular}




$\begin{array}{llrll}\mathrm{C}(22 \mathrm{~B}) & 5000 & 7880(4) & 7500 & 105(4) \\ \mathrm{C}(23 \mathrm{~B}) & 5242(1) & 8227(4) & 7409(6) & 116(3) \\ \mathrm{C}(24 \mathrm{~B}) & 5254(2) & 8947(5) & 7413(9) & 159(3) \\ \mathrm{C}(25 \mathrm{~B}) & 5000 & 9300(7) & 7500 & 161(3) \\ \mathrm{C}(26 \mathrm{~B}) & 5544(3) & 9244(6) & 7129(11) & 161(3) \\ \mathrm{C}(27 \mathrm{~B}) & 5789(5) & 9122(9) & 7796(12) & 160(3) \\ \mathrm{C}(28 \mathrm{~B}) & 5473(4) & 10029(7) & 7057(13) & 162(3) \\ \mathrm{C}(29 \mathrm{~B}) & 5634(4) & 8982(9) & 6319(11) & 167(3) \\ \mathrm{C}\left(26^{\prime}\right) & 5509(3) & 9416(6) & 7391(10) & 161(3) \\ \mathrm{C}\left(27^{\prime}\right) & 5576(4) & 10145(7) & 7731(12) & 163(3) \\ \mathrm{C}\left(28^{\prime}\right) & 5439(4) & 9460(9) & 6452(10) & 167(3) \\ \mathrm{C}\left(29^{\prime}\right) & 5771(4) & 8953(8) & 7614(14) & 159(3) \\ \mathrm{C}(30) & 3748(1) & 1092(2) & 9223(2) & 37(1) \\ \mathrm{C}(31) & 3436(1) & 1285(3) & 9142(3) & 51(1) \\ \mathrm{C}(32) & 3850(1) & 933(3) & 10115(3) & 54(1) \\ \mathrm{C}(33) & 3782(1) & 439(2) & 8718(3) & 56(1)\end{array}$

Table S5. Bond lengths $[\AA ̊]$ and angles $\left[^{\circ}\right]$ for FR-1.

$\begin{array}{llll}\mathrm{C}(1 \mathrm{~A})-\mathrm{C}(14 \mathrm{~A}) & 1.404(5) & \mathrm{C}(1 \mathrm{~A})-\mathrm{C}(2 \mathrm{~A}) & 1.415(5) \\ \mathrm{C}(1 \mathrm{~A})-\mathrm{C}(15 \mathrm{~A}) & 1.493(4) & \mathrm{C}(2 \mathrm{~A})-\mathrm{C}(3 \mathrm{~A}) & 1.425(5) \\ \mathrm{C}(2 \mathrm{~A})-\mathrm{C}(7 \mathrm{~A}) & 1.439(4) & \mathrm{C}(3 \mathrm{~A})-\mathrm{C}(4 \mathrm{~A}) & 1.364(5) \\ \mathrm{C}(3 \mathrm{~A})-\mathrm{H}(3 \mathrm{~A}) & 0.9500 & \mathrm{C}(4 \mathrm{~A})-\mathrm{C}(5 \mathrm{~A}) & 1.418(5) \\ \mathrm{C}(4 \mathrm{~A})-\mathrm{H}(4 \mathrm{~A}) & 0.9500 & \mathrm{C}(5 \mathrm{~A})-\mathrm{C}(6 \mathrm{~A}) & 1.358(5) \\ \mathrm{C}(5 \mathrm{~A})-\mathrm{H}(5 \mathrm{~A}) & 0.9500 & \mathrm{C}(6 \mathrm{~A})-\mathrm{C}(7 \mathrm{~A}) & 1.433(5) \\ \mathrm{C}(6 \mathrm{~A})-\mathrm{H}(6 \mathrm{~A}) & 0.9500 & \mathrm{C}(7 \mathrm{~A})-\mathrm{C}(8 \mathrm{~A}) & 1.408(5) \\ \mathrm{C}(8 \mathrm{~A})-\mathrm{C}(9 \mathrm{~A}) & 1.409(5) & \mathrm{C}(8 \mathrm{~A})-\mathrm{C}(21 \mathrm{~A}) & 1.475(4) \\ \mathrm{C}(9 \mathrm{~A})-\mathrm{C}(10 \mathrm{~A}) & 1.433(5) & \mathrm{C}(9 \mathrm{~A})-\mathrm{C}(14 \mathrm{~A}) & 1.435(4) \\ \mathrm{C}(10 \mathrm{~A})-\mathrm{C}(11 \mathrm{~A}) & 1.360(5) & \mathrm{C}(10 \mathrm{~A})-\mathrm{H}(10 \mathrm{~A}) & 0.9500 \\ \mathrm{C}(11 \mathrm{~A})-\mathrm{C}(12 \mathrm{~A}) & 1.402(5) & \mathrm{C}(11 \mathrm{~A})-\mathrm{H}(11 \mathrm{~A}) & 0.9500 \\ \mathrm{C}(12 \mathrm{~A})-\mathrm{C}(13 \mathrm{~A}) & 1.356(5) & \mathrm{C}(12 \mathrm{~A})-\mathrm{H}(12 \mathrm{~A}) & 0.9500 \\ \mathrm{C}(13 \mathrm{~A})-\mathrm{C}(14 \mathrm{~A}) & 1.441(5) & \mathrm{C}(13 \mathrm{~A})-\mathrm{H}(13 \mathrm{~A}) & 0.9500 \\ \mathrm{C}(15 \mathrm{~A})-\mathrm{C}(16 \mathrm{~A}) & 1.381(5) & \mathrm{C}(15 \mathrm{~A})-\mathrm{C}(20 \mathrm{~A}) & 1.393(5) \\ \mathrm{C}(16 \mathrm{~A})-\mathrm{C}(17 \mathrm{~A}) & 1.402(5) & \mathrm{C}(16 \mathrm{~A})-\mathrm{H}(16 \mathrm{~A}) & 0.9500\end{array}$




\begin{tabular}{|c|c|c|c|}
\hline $\mathrm{C}(17 \mathrm{~A})-\mathrm{C}(18 \mathrm{~A})$ & $1.392(5)$ & $\mathrm{C}(17 \mathrm{~A})-\mathrm{C}(46 \mathrm{~A})$ & $1.529(5)$ \\
\hline $\mathrm{C}(18 \mathrm{~A})-\mathrm{C}(19 \mathrm{~A})$ & $1.395(5)$ & $\mathrm{C}(18 \mathrm{~A})-\mathrm{H}(18 \mathrm{~A})$ & 0.9500 \\
\hline $\mathrm{C}(19 \mathrm{~A})-\mathrm{C}(20 \mathrm{~A})$ & $1.387(5)$ & $C(19 A)-C(50 A)$ & $1.534(5)$ \\
\hline$C(20 A)-H(20 A)$ & 0.9500 & $\mathrm{C}(21 \mathrm{~A})-\mathrm{C}(22 \mathrm{~A})$ & $1.431(5)$ \\
\hline$C(21 \mathrm{~A})-\mathrm{C}(33 \mathrm{~A})$ & $1.438(5)$ & $\mathrm{C}(22 \mathrm{~A})-\mathrm{C}(23 \mathrm{~A})$ & $1.403(5)$ \\
\hline $\mathrm{C}(22 \mathrm{~A})-\mathrm{C}(27 \mathrm{~A})$ & $1.423(5)$ & $\mathrm{C}(23 \mathrm{~A})-\mathrm{C}(24 \mathrm{~A})$ & $1.377(5)$ \\
\hline $\mathrm{C}(23 \mathrm{~A})-\mathrm{H}(23 \mathrm{~A})$ & 0.9500 & $C(24 A)-C(25 A)$ & $1.411(5)$ \\
\hline $\mathrm{C}(24 \mathrm{~A})-\mathrm{H}(24 \mathrm{~A})$ & 0.9500 & $C(25 A)-C(26 A)$ & $1.403(5)$ \\
\hline $\mathrm{C}(25 \mathrm{~A})-\mathrm{C}(34 \mathrm{~A})$ & $1.470(5)$ & $C(26 A)-C(27 A)$ & $1.370(5)$ \\
\hline $\mathrm{C}(26 \mathrm{~A})-\mathrm{H}(26 \mathrm{~A})$ & 0.9500 & $\mathrm{C}(27 \mathrm{~A})-\mathrm{C}(28 \mathrm{~A})$ & $1.470(5)$ \\
\hline $\mathrm{C}(28 \mathrm{~A})-\mathrm{C}(29 \mathrm{~A})$ & $1.384(5)$ & $\mathrm{C}(28 \mathrm{~A})-\mathrm{C}(33 \mathrm{~A})$ & $1.420(4)$ \\
\hline$C(29 A)-C(30 A)$ & $1.408(5)$ & $\mathrm{C}(29 \mathrm{~A})-\mathrm{H}(29 \mathrm{~A})$ & 0.9500 \\
\hline$C(30 A)-C(31 A)$ & $1.404(5)$ & $C(30 A)-C(40 A)$ & $1.479(5)$ \\
\hline$C(31 \mathrm{~A})-\mathrm{C}(32 \mathrm{~A})$ & $1.375(5)$ & $\mathrm{C}(31 \mathrm{~A})-\mathrm{H}(31 \mathrm{~A})$ & 0.9500 \\
\hline$C(32 \mathrm{~A})-\mathrm{C}(33 \mathrm{~A})$ & $1.398(5)$ & $\mathrm{C}(32 \mathrm{~A})-\mathrm{H}(32 \mathrm{~A})$ & 0.9500 \\
\hline $\mathrm{C}(34 \mathrm{~A})-\mathrm{C}(39 \mathrm{~A})$ & $1.381(5)$ & $\mathrm{C}(34 \mathrm{~A})-\mathrm{C}(35 \mathrm{~A})$ & $1.398(5)$ \\
\hline $\mathrm{C}(35 \mathrm{~A})-\mathrm{C}(36 \mathrm{~A})$ & $1.381(6)$ & $\mathrm{C}(35 \mathrm{~A})-\mathrm{H}(35 \mathrm{~A})$ & 0.9500 \\
\hline$C(36 \mathrm{~A})-\mathrm{C}(37 \mathrm{~A})$ & $1.372(7)$ & $\mathrm{C}(36 \mathrm{~A})-\mathrm{H}(36 \mathrm{~A})$ & 0.9500 \\
\hline $\mathrm{C}(37 \mathrm{~A})-\mathrm{C}(38 \mathrm{~A})$ & $1.402(6)$ & $C(37 \mathrm{~A})-\mathrm{C}(54 \mathrm{~A})$ & $1.504(10)$ \\
\hline $\mathrm{C}(37 \mathrm{~A})-\mathrm{C}\left(54^{\prime}\right)$ & $1.575(10)$ & $\mathrm{C}(38 \mathrm{~A})-\mathrm{C}(39 \mathrm{~A})$ & $1.387(6)$ \\
\hline $\mathrm{C}(38 \mathrm{~A})-\mathrm{H}(38 \mathrm{~A})$ & 0.9500 & $\mathrm{C}(39 \mathrm{~A})-\mathrm{H}(39 \mathrm{~A})$ & 0.9500 \\
\hline$C(40 A)-C(45 A)$ & $1.390(5)$ & $C(40 A)-C(41 A)$ & $1.399(5)$ \\
\hline $\mathrm{C}(41 \mathrm{~A})-\mathrm{C}(42 \mathrm{~A})$ & $1.379(5)$ & $\mathrm{C}(41 \mathrm{~A})-\mathrm{H}(41 \mathrm{~A})$ & 0.9500 \\
\hline$C(42 A)-C(43 A)$ & $1.394(5)$ & $\mathrm{C}(42 \mathrm{~A})-\mathrm{H}(42 \mathrm{~A})$ & 0.9500 \\
\hline$C(43 \mathrm{~A})-\mathrm{C}(44 \mathrm{~A})$ & $1.403(5)$ & $C(43 A)-C(58 A)$ & $1.518(9)$ \\
\hline $\mathrm{C}(43 \mathrm{~A})-\mathrm{C}\left(58^{\prime}\right)$ & $1.535(14)$ & $\mathrm{C}(44 \mathrm{~A})-\mathrm{C}(45 \mathrm{~A})$ & $1.376(5)$ \\
\hline $\mathrm{C}(44 \mathrm{~A})-\mathrm{H}(44 \mathrm{~A})$ & 0.9500 & $\mathrm{C}(45 \mathrm{~A})-\mathrm{H}(45 \mathrm{~A})$ & 0.9500 \\
\hline$C(46 A)-C(49 A)$ & $1.529(5)$ & $C(46 A)-C(48 A)$ & $1.540(5)$ \\
\hline$C(46 A)-C(47 A)$ & $1.545(5)$ & $\mathrm{C}(47 \mathrm{~A})-\mathrm{H}(47 \mathrm{~A})$ & 0.9800 \\
\hline $\mathrm{C}(47 \mathrm{~A})-\mathrm{H}(47 \mathrm{~B})$ & 0.9800 & $\mathrm{C}(47 \mathrm{~A})-\mathrm{H}(47 \mathrm{C})$ & 0.9800 \\
\hline $\mathrm{C}(48 \mathrm{~A})-\mathrm{H}(48 \mathrm{~A})$ & 0.9800 & $\mathrm{C}(48 \mathrm{~A})-\mathrm{H}(48 \mathrm{~B})$ & 0.9800 \\
\hline $\mathrm{C}(48 \mathrm{~A})-\mathrm{H}(48 \mathrm{C})$ & 0.9800 & $\mathrm{C}(49 \mathrm{~A})-\mathrm{H}(49 \mathrm{~A})$ & 0.9800 \\
\hline C(49A)-H(49B) & 0.9800 & $\mathrm{C}(49 \mathrm{~A})-\mathrm{H}(49 \mathrm{C})$ & 0.9800 \\
\hline$C(50 A)-C(51 A)$ & $1.528(4)$ & $C(50 A)-C(53 A)$ & $1.537(4)$ \\
\hline$C(50 A)-C(52 A)$ & $1.542(4)$ & $\mathrm{C}(51 \mathrm{~A})-\mathrm{H}(51 \mathrm{~A})$ & 0.9800 \\
\hline $\mathrm{C}(51 \mathrm{~A})-\mathrm{H}(51 \mathrm{~B})$ & 0.9800 & $\mathrm{C}(51 \mathrm{~A})-\mathrm{H}(51 \mathrm{C})$ & 0.9800 \\
\hline
\end{tabular}




\begin{tabular}{|c|c|c|c|}
\hline $\mathrm{C}(52 \mathrm{~A})-\mathrm{H}(52 \mathrm{~A})$ & 0.9800 & $\mathrm{C}(52 \mathrm{~A})-\mathrm{H}(52 \mathrm{~B})$ & 0.9800 \\
\hline $\mathrm{C}(52 \mathrm{~A})-\mathrm{H}(52 \mathrm{C})$ & 0.9800 & $\mathrm{C}(53 \mathrm{~A})-\mathrm{H}(53 \mathrm{~A})$ & 0.9800 \\
\hline $\mathrm{C}(53 \mathrm{~A})-\mathrm{H}(53 \mathrm{~B})$ & 0.9800 & $\mathrm{C}(53 \mathrm{~A})-\mathrm{H}(53 \mathrm{C})$ & 0.9800 \\
\hline $\mathrm{C}(54 \mathrm{~A})-\mathrm{C}(55 \mathrm{~A})$ & $1.526(5)$ & $\mathrm{C}(54 \mathrm{~A})-\mathrm{C}(57 \mathrm{~A})$ & $1.539(5)$ \\
\hline $\mathrm{C}(54 \mathrm{~A})-\mathrm{C}(56 \mathrm{~A})$ & $1.545(5)$ & $\mathrm{C}(55 \mathrm{~A})-\mathrm{H}(55 \mathrm{~A})$ & 0.9800 \\
\hline $\mathrm{C}(55 \mathrm{~A})-\mathrm{H}(55 \mathrm{~B})$ & 0.9800 & $\mathrm{C}(55 \mathrm{~A})-\mathrm{H}(55 \mathrm{C})$ & 0.9800 \\
\hline $\mathrm{C}(56 \mathrm{~A})-\mathrm{H}(56 \mathrm{~A})$ & 0.9800 & $\mathrm{C}(56 \mathrm{~A})-\mathrm{H}(56 \mathrm{~B})$ & 0.9800 \\
\hline $\mathrm{C}(56 \mathrm{~A})-\mathrm{H}(56 \mathrm{C})$ & 0.9800 & $\mathrm{C}(57 \mathrm{~A})-\mathrm{H}(57 \mathrm{~A})$ & 0.9800 \\
\hline $\mathrm{C}(57 \mathrm{~A})-\mathrm{H}(57 \mathrm{~B})$ & 0.9800 & $\mathrm{C}(57 \mathrm{~A})-\mathrm{H}(57 \mathrm{C})$ & 0.9800 \\
\hline $\mathrm{C}\left(54^{\prime}\right)-\mathrm{C}\left(55^{\prime}\right)$ & $1.526(5)$ & $\mathrm{C}\left(54^{\prime}\right)-\mathrm{C}\left(57^{\prime}\right)$ & $1.544(5)$ \\
\hline $\mathrm{C}\left(54^{\prime}\right)-\mathrm{C}\left(56^{\prime}\right)$ & $1.546(5)$ & $\mathrm{C}\left(55^{\prime}\right)-\mathrm{H}(55 \mathrm{D})$ & 0.9800 \\
\hline $\mathrm{C}\left(55^{\prime}\right)-\mathrm{H}(55 \mathrm{E})$ & 0.9800 & $\mathrm{C}\left(55^{\prime}\right)-\mathrm{H}(55 \mathrm{~F})$ & 0.9800 \\
\hline $\mathrm{C}\left(56^{\prime}\right)-\mathrm{H}(56 \mathrm{D})$ & 0.9800 & $\mathrm{C}\left(56^{\prime}\right)-\mathrm{H}(56 \mathrm{E})$ & 0.9800 \\
\hline $\mathrm{C}\left(56^{\prime}\right)-\mathrm{H}(56 \mathrm{~F})$ & 0.9800 & $\mathrm{C}\left(57^{\prime}\right)-\mathrm{H}(57 \mathrm{D})$ & 0.9800 \\
\hline $\mathrm{C}\left(57^{\prime}\right)-\mathrm{H}(57 \mathrm{E})$ & 0.9800 & $\mathrm{C}\left(57^{\prime}\right)-\mathrm{H}(57 \mathrm{~F})$ & 0.9800 \\
\hline $\mathrm{C}(58 \mathrm{~A})-\mathrm{C}(59 \mathrm{~A})$ & $1.525(5)$ & $\mathrm{C}(58 \mathrm{~A})-\mathrm{C}(60 \mathrm{~A})$ & $1.543(5)$ \\
\hline $\mathrm{C}(58 \mathrm{~A})-\mathrm{C}(61 \mathrm{~A})$ & $1.544(5)$ & $\mathrm{C}(59 \mathrm{~A})-\mathrm{H}(59 \mathrm{~A})$ & 0.9800 \\
\hline C(59A)-H(59B) & 0.9800 & $\mathrm{C}(59 \mathrm{~A})-\mathrm{H}(59 \mathrm{C})$ & 0.9800 \\
\hline $\mathrm{C}(60 \mathrm{~A})-\mathrm{H}(60 \mathrm{~A})$ & 0.9800 & $\mathrm{C}(60 \mathrm{~A})-\mathrm{H}(60 \mathrm{~B})$ & 0.9800 \\
\hline $\mathrm{C}(60 \mathrm{~A})-\mathrm{H}(60 \mathrm{C})$ & 0.9800 & $\mathrm{C}(61 \mathrm{~A})-\mathrm{H}(61 \mathrm{~A})$ & 0.9800 \\
\hline $\mathrm{C}(61 \mathrm{~A})-\mathrm{H}(61 \mathrm{~B})$ & 0.9800 & $\mathrm{C}(61 \mathrm{~A})-\mathrm{H}(61 \mathrm{C})$ & 0.9800 \\
\hline $\mathrm{C}\left(58^{\prime}\right)-\mathrm{C}\left(59^{\prime}\right)$ & $1.526(5)$ & $\mathrm{C}\left(58^{\prime}\right)-\mathrm{C}\left(61^{\prime}\right)$ & $1.542(5)$ \\
\hline $\mathrm{C}\left(58^{\prime}\right)-\mathrm{C}\left(60^{\prime}\right)$ & $1.544(5)$ & $\mathrm{C}\left(59^{\prime}\right)-\mathrm{H}(59 \mathrm{D})$ & 0.9800 \\
\hline $\mathrm{C}\left(59^{\prime}\right)-\mathrm{H}(59 \mathrm{E})$ & 0.9800 & $\mathrm{C}\left(59^{\prime}\right)-\mathrm{H}(59 \mathrm{~F})$ & 0.9800 \\
\hline $\mathrm{C}\left(60^{\prime}\right)-\mathrm{H}(60 \mathrm{D})$ & 0.9800 & $\mathrm{C}\left(60^{\prime}\right)-\mathrm{H}(60 \mathrm{E})$ & 0.9800 \\
\hline $\mathrm{C}\left(60^{\prime}\right)-\mathrm{H}(60 \mathrm{~F})$ & 0.9800 & $\mathrm{C}\left(61^{\prime}\right)-\mathrm{H}(61 \mathrm{D})$ & 0.9800 \\
\hline$C\left(61^{\prime}\right)-H(61 E)$ & 0.9800 & $\mathrm{C}\left(61^{\prime}\right)-\mathrm{H}(61 \mathrm{~F})$ & 0.9800 \\
\hline $\mathrm{C}(1 \mathrm{~B})-\mathrm{C}(2 \mathrm{~B})$ & $1.427(5)$ & $\mathrm{C}(1 \mathrm{~B})-\mathrm{C}(2 \mathrm{~B}) \# 1$ & $1.427(5)$ \\
\hline$C(1 B)-C(14 B)$ & $1.478(8)$ & $\mathrm{C}(2 \mathrm{~B})-\mathrm{C}(3 \mathrm{~B})$ & $1.398(5)$ \\
\hline $\mathrm{C}(2 \mathrm{~B})-\mathrm{C}(7 \mathrm{~B})$ & $1.424(5)$ & $\mathrm{C}(3 \mathrm{~B})-\mathrm{C}(4 \mathrm{~B})$ & $1.370(5)$ \\
\hline $\mathrm{C}(3 \mathrm{~B})-\mathrm{H}(3 \mathrm{~B})$ & 0.9500 & $C(4 B)-C(5 B)$ & $1.407(5)$ \\
\hline $\mathrm{C}(4 \mathrm{~B})-\mathrm{H}(4 \mathrm{~B})$ & 0.9500 & $C(5 B)-C(6 B)$ & $1.401(5)$ \\
\hline$C(5 B)-C(8 B)$ & $1.489(5)$ & $\mathrm{C}(6 \mathrm{~B})-\mathrm{C}(7 \mathrm{~B})$ & $1.366(5)$ \\
\hline $\mathrm{C}(6 \mathrm{~B})-\mathrm{H}(6 \mathrm{~B})$ & 0.9500 & $\mathrm{C}(7 \mathrm{~B})-\mathrm{C}(7 \mathrm{~B}) \# 1$ & $1.464(7)$ \\
\hline $\mathrm{C}(8 \mathrm{~B})-\mathrm{C}(13 \mathrm{~B})$ & $1.392(5)$ & $\mathrm{C}(8 \mathrm{~B})-\mathrm{C}(9 \mathrm{~B})$ & $1.394(5)$ \\
\hline
\end{tabular}




\begin{tabular}{|c|c|c|c|}
\hline $\mathrm{C}(9 \mathrm{~B})-\mathrm{C}(10 \mathrm{~B})$ & $1.380(5)$ & $\mathrm{C}(9 \mathrm{~B})-\mathrm{H}(9 \mathrm{~B})$ & 0.9500 \\
\hline $\mathrm{C}(10 \mathrm{~B})-\mathrm{C}(11 \mathrm{~B})$ & $1.383(5)$ & $\mathrm{C}(10 \mathrm{~B})-\mathrm{H}(10 \mathrm{~B})$ & 0.9500 \\
\hline $\mathrm{C}(11 \mathrm{~B})-\mathrm{C}(12 \mathrm{~B})$ & $1.398(5)$ & $\mathrm{C}(11 \mathrm{~B})-\mathrm{C}(30)$ & $1.528(5)$ \\
\hline $\mathrm{C}(12 \mathrm{~B})-\mathrm{C}(13 \mathrm{~B})$ & $1.377(6)$ & $\mathrm{C}(12 \mathrm{~B})-\mathrm{H}(12 \mathrm{~B})$ & 0.9500 \\
\hline C(13B)-H(13B) & 0.9500 & $C(14 B)-C(15 B) \# 1$ & $1.410(5)$ \\
\hline$C(14 B)-C(15 B)$ & $1.410(5)$ & $\mathrm{C}(15 \mathrm{~B})-\mathrm{C}(16 \mathrm{~B})$ & $1.422(7)$ \\
\hline$C(15 B)-C(20 B)$ & $1.434(7)$ & $\mathrm{C}(16 \mathrm{~B})-\mathrm{C}(21 \mathrm{~B})$ & $1.410(7)$ \\
\hline $\mathrm{C}(16 \mathrm{~B})-\mathrm{C}(17 \mathrm{~B})$ & $1.469(8)$ & $\mathrm{C}(17 \mathrm{~B})-\mathrm{C}(18 \mathrm{~B})$ & $1.357(9)$ \\
\hline C(17B)-H(17B) & 0.9500 & $\mathrm{C}(18 \mathrm{~B})-\mathrm{C}(19 \mathrm{~B})$ & $1.383(9)$ \\
\hline $\mathrm{C}(18 \mathrm{~B})-\mathrm{H}(18 \mathrm{~B})$ & 0.9500 & $C(19 B)-C(20 B)$ & $1.364(7)$ \\
\hline C(19B)-H(19B) & 0.9500 & $\mathrm{C}(20 \mathrm{~B})-\mathrm{H}(20 \mathrm{~B})$ & 0.9500 \\
\hline$C(21 B)-C(16 B) \# 1$ & $1.410(7)$ & $\mathrm{C}(21 \mathrm{~B})-\mathrm{C}(22 \mathrm{~B})$ & $1.497(10)$ \\
\hline $\mathrm{C}(22 \mathrm{~B})-\mathrm{C}(23 \mathrm{~B}) \# 1$ & $1.361(7)$ & $\mathrm{C}(22 \mathrm{~B})-\mathrm{C}(23 \mathrm{~B})$ & $1.361(7)$ \\
\hline$C(23 B)-C(24 B)$ & $1.382(11)$ & $\mathrm{C}(23 \mathrm{~B})-\mathrm{H}(23 \mathrm{~B})$ & 0.9500 \\
\hline$C(24 B)-C(25 B)$ & $1.414(11)$ & $\mathrm{C}(24 \mathrm{~B})-\mathrm{C}\left(26^{\prime}\right)$ & $1.520(15)$ \\
\hline$C(24 B)-C(26 B)$ & $1.624(18)$ & $\mathrm{C}(25 \mathrm{~B})-\mathrm{C}(24 \mathrm{~B}) \# 1$ & $1.414(11)$ \\
\hline $\mathrm{C}(25 \mathrm{~B})-\mathrm{H}(25 \mathrm{~B})$ & 0.9500 & $\mathrm{C}(26 \mathrm{~B})-\mathrm{C}(27 \mathrm{~B})$ & $1.524(6)$ \\
\hline$C(26 B)-C(29 B)$ & $1.543(6)$ & $\mathrm{C}(26 \mathrm{~B})-\mathrm{C}(28 \mathrm{~B})$ & $1.544(5)$ \\
\hline $\mathrm{C}(27 \mathrm{~B})-\mathrm{H}(27 \mathrm{~A})$ & 0.9800 & $\mathrm{C}(27 \mathrm{~B})-\mathrm{H}(27 \mathrm{~B})$ & 0.9800 \\
\hline C(27B)-H(27C) & 0.9800 & $\mathrm{C}(28 \mathrm{~B})-\mathrm{H}(28 \mathrm{~A})$ & 0.9800 \\
\hline $\mathrm{C}(28 \mathrm{~B})-\mathrm{H}(28 \mathrm{~B})$ & 0.9800 & $\mathrm{C}(28 \mathrm{~B})-\mathrm{H}(28 \mathrm{C})$ & 0.9800 \\
\hline C(29B)-H(29B) & 0.9800 & $\mathrm{C}(29 \mathrm{~B})-\mathrm{H}(29 \mathrm{C})$ & 0.9800 \\
\hline C(29B)-H(29D) & 0.9800 & $\mathrm{C}\left(26^{\prime}\right)-\mathrm{C}\left(27^{\prime}\right)$ & $1.525(6)$ \\
\hline $\mathrm{C}\left(26^{\prime}\right)-\mathrm{C}\left(29^{\prime}\right)$ & $1.543(6)$ & $\mathrm{C}\left(26^{\prime}\right)-\mathrm{C}\left(28^{\prime}\right)$ & $1.549(5)$ \\
\hline $\mathrm{C}\left(27^{\prime}\right)-\mathrm{H}(27 \mathrm{D})$ & 0.9800 & $\mathrm{C}\left(27^{\prime}\right)-\mathrm{H}(27 \mathrm{E})$ & 0.9800 \\
\hline $\mathrm{C}\left(27^{\prime}\right)-\mathrm{H}(27 \mathrm{~F})$ & 0.9800 & $\mathrm{C}\left(28^{\prime}\right)-\mathrm{H}(28 \mathrm{D})$ & 0.9800 \\
\hline $\mathrm{C}\left(28^{\prime}\right)-\mathrm{H}(28 \mathrm{E})$ & 0.9800 & $\mathrm{C}\left(28^{\prime}\right)-\mathrm{H}(28 \mathrm{~F})$ & 0.9800 \\
\hline $\mathrm{C}\left(29^{\prime}\right)-\mathrm{H}(29 \mathrm{E})$ & 0.9800 & $\mathrm{C}\left(2^{\prime}\right)-\mathrm{H}(29 \mathrm{~F})$ & 0.9800 \\
\hline $\mathrm{C}\left(29^{\prime}\right)-\mathrm{H}(29 \mathrm{G})$ & 0.9800 & $\mathrm{C}(30)-\mathrm{C}(33)$ & $1.524(6)$ \\
\hline$C(30)-C(32)$ & $1.526(6)$ & $C(30)-C(31)$ & $1.531(6)$ \\
\hline $\mathrm{C}(31)-\mathrm{H}(31 \mathrm{~B})$ & 0.9800 & $\mathrm{C}(31)-\mathrm{H}(31 \mathrm{C})$ & 0.9800 \\
\hline $\mathrm{C}(31)-\mathrm{H}(31 \mathrm{D})$ & 0.9800 & $\mathrm{C}(32)-\mathrm{H}(32 \mathrm{~B})$ & 0.9800 \\
\hline $\mathrm{C}(32)-\mathrm{H}(32 \mathrm{C})$ & 0.9800 & $\mathrm{C}(32)-\mathrm{H}(32 \mathrm{D})$ & 0.9800 \\
\hline $\mathrm{C}(33)-\mathrm{H}(33 \mathrm{~A})$ & 0.9800 & $\mathrm{C}(33)-\mathrm{H}(33 \mathrm{~B})$ & 0.9800 \\
\hline $\mathrm{C}(33)-\mathrm{H}(33 \mathrm{C})$ & 0.9800 & & \\
\hline
\end{tabular}




\begin{tabular}{|c|c|c|c|}
\hline$C(14 A)-C(1 A)-C(2 A)$ & $120.2(3)$ & $C(14 A)-C(1 A)-C(15 A)$ & $119.5(3)$ \\
\hline $\mathrm{C}(2 \mathrm{~A})-\mathrm{C}(1 \mathrm{~A})-\mathrm{C}(15 \mathrm{~A})$ & $120.2(3)$ & $\mathrm{C}(1 \mathrm{~A})-\mathrm{C}(2 \mathrm{~A})-\mathrm{C}(3 \mathrm{~A})$ & $121.7(3)$ \\
\hline$C(1 \mathrm{~A})-\mathrm{C}(2 \mathrm{~A})-\mathrm{C}(7 \mathrm{~A})$ & $119.4(3)$ & $C(3 A)-C(2 A)-C(7 A)$ & $118.9(3)$ \\
\hline$C(4 A)-C(3 A)-C(2 A)$ & $120.7(3)$ & $\mathrm{C}(4 \mathrm{~A})-\mathrm{C}(3 \mathrm{~A})-\mathrm{H}(3 \mathrm{~A})$ & 119.7 \\
\hline $\mathrm{C}(2 \mathrm{~A})-\mathrm{C}(3 \mathrm{~A})-\mathrm{H}(3 \mathrm{~A})$ & 119.7 & $\mathrm{C}(3 \mathrm{~A})-\mathrm{C}(4 \mathrm{~A})-\mathrm{C}(5 \mathrm{~A})$ & $120.7(3)$ \\
\hline $\mathrm{C}(3 \mathrm{~A})-\mathrm{C}(4 \mathrm{~A})-\mathrm{H}(4 \mathrm{~A})$ & 119.6 & $\mathrm{C}(5 \mathrm{~A})-\mathrm{C}(4 \mathrm{~A})-\mathrm{H}(4 \mathrm{~A})$ & 119.6 \\
\hline$C(6 A)-C(5 A)-C(4 A)$ & $120.3(3)$ & $\mathrm{C}(6 \mathrm{~A})-\mathrm{C}(5 \mathrm{~A})-\mathrm{H}(5 \mathrm{~A})$ & 119.8 \\
\hline $\mathrm{C}(4 \mathrm{~A})-\mathrm{C}(5 \mathrm{~A})-\mathrm{H}(5 \mathrm{~A})$ & 119.8 & $\mathrm{C}(5 \mathrm{~A})-\mathrm{C}(6 \mathrm{~A})-\mathrm{C}(7 \mathrm{~A})$ & $121.2(3)$ \\
\hline $\mathrm{C}(5 \mathrm{~A})-\mathrm{C}(6 \mathrm{~A})-\mathrm{H}(6 \mathrm{~A})$ & 119.4 & $\mathrm{C}(7 \mathrm{~A})-\mathrm{C}(6 \mathrm{~A})-\mathrm{H}(6 \mathrm{~A})$ & 119.4 \\
\hline $\mathrm{C}(8 \mathrm{~A})-\mathrm{C}(7 \mathrm{~A})-\mathrm{C}(6 \mathrm{~A})$ & $121.7(3)$ & $\mathrm{C}(8 \mathrm{~A})-\mathrm{C}(7 \mathrm{~A})-\mathrm{C}(2 \mathrm{~A})$ & $120.2(3)$ \\
\hline$C(6 A)-C(7 A)-C(2 A)$ & $118.1(3)$ & $\mathrm{C}(7 \mathrm{~A})-\mathrm{C}(8 \mathrm{~A})-\mathrm{C}(9 \mathrm{~A})$ & $120.1(3)$ \\
\hline $\mathrm{C}(7 \mathrm{~A})-\mathrm{C}(8 \mathrm{~A})-\mathrm{C}(21 \mathrm{~A})$ & $120.5(3)$ & $\mathrm{C}(9 \mathrm{~A})-\mathrm{C}(8 \mathrm{~A})-\mathrm{C}(21 \mathrm{~A})$ & 119.4(3) \\
\hline $\mathrm{C}(8 \mathrm{~A})-\mathrm{C}(9 \mathrm{~A})-\mathrm{C}(10 \mathrm{~A})$ & $121.6(3)$ & $\mathrm{C}(8 \mathrm{~A})-\mathrm{C}(9 \mathrm{~A})-\mathrm{C}(14 \mathrm{~A})$ & $119.9(3)$ \\
\hline$C(10 A)-C(9 A)-C(14 A)$ & $118.4(3)$ & $C(11 A)-C(10 A)-C(9 A)$ & $121.3(3)$ \\
\hline $\mathrm{C}(11 \mathrm{~A})-\mathrm{C}(10 \mathrm{~A})-\mathrm{H}(10 \mathrm{~A})$ & 119.3 & $\mathrm{C}(9 \mathrm{~A})-\mathrm{C}(10 \mathrm{~A})-\mathrm{H}(10 \mathrm{~A})$ & 119.3 \\
\hline $\mathrm{C}(10 \mathrm{~A})-\mathrm{C}(11 \mathrm{~A})-\mathrm{C}(12 \mathrm{~A})$ & $120.1(3)$ & $\mathrm{C}(10 \mathrm{~A})-\mathrm{C}(11 \mathrm{~A})-\mathrm{H}(11 \mathrm{~A})$ & 119.9 \\
\hline $\mathrm{C}(12 \mathrm{~A})-\mathrm{C}(11 \mathrm{~A})-\mathrm{H}(11 \mathrm{~A})$ & 119.9 & $\mathrm{C}(13 \mathrm{~A})-\mathrm{C}(12 \mathrm{~A})-\mathrm{C}(11 \mathrm{~A})$ & $121.5(3)$ \\
\hline $\mathrm{C}(13 \mathrm{~A})-\mathrm{C}(12 \mathrm{~A})-\mathrm{H}(12 \mathrm{~A})$ & 119.3 & $\mathrm{C}(11 \mathrm{~A})-\mathrm{C}(12 \mathrm{~A})-\mathrm{H}(12 \mathrm{~A})$ & 119.3 \\
\hline $\mathrm{C}(12 \mathrm{~A})-\mathrm{C}(13 \mathrm{~A})-\mathrm{C}(14 \mathrm{~A})$ & $120.8(3)$ & $\mathrm{C}(12 \mathrm{~A})-\mathrm{C}(13 \mathrm{~A})-\mathrm{H}(13 \mathrm{~A})$ & 119.6 \\
\hline $\mathrm{C}(14 \mathrm{~A})-\mathrm{C}(13 \mathrm{~A})-\mathrm{H}(13 \mathrm{~A})$ & 119.6 & $\mathrm{C}(1 \mathrm{~A})-\mathrm{C}(14 \mathrm{~A})-\mathrm{C}(9 \mathrm{~A})$ & $120.2(3)$ \\
\hline $\mathrm{C}(1 \mathrm{~A})-\mathrm{C}(14 \mathrm{~A})-\mathrm{C}(13 \mathrm{~A})$ & $121.9(3)$ & $\mathrm{C}(9 \mathrm{~A})-\mathrm{C}(14 \mathrm{~A})-\mathrm{C}(13 \mathrm{~A})$ & $117.9(3)$ \\
\hline $\mathrm{C}(16 \mathrm{~A})-\mathrm{C}(15 \mathrm{~A})-\mathrm{C}(20 \mathrm{~A})$ & $119.5(3)$ & $C(16 A)-C(15 A)-C(1 A)$ & $122.2(3)$ \\
\hline$C(20 A)-C(15 A)-C(1 A)$ & $118.3(3)$ & $\mathrm{C}(15 \mathrm{~A})-\mathrm{C}(16 \mathrm{~A})-\mathrm{C}(17 \mathrm{~A})$ & $121.3(3)$ \\
\hline $\mathrm{C}(15 \mathrm{~A})-\mathrm{C}(16 \mathrm{~A})-\mathrm{H}(16 \mathrm{~A})$ & 119.3 & $\mathrm{C}(17 \mathrm{~A})-\mathrm{C}(16 \mathrm{~A})-\mathrm{H}(16 \mathrm{~A})$ & 119.3 \\
\hline $\mathrm{C}(18 \mathrm{~A})-\mathrm{C}(17 \mathrm{~A})-\mathrm{C}(16 \mathrm{~A})$ & $117.5(3)$ & $\mathrm{C}(18 \mathrm{~A})-\mathrm{C}(17 \mathrm{~A})-\mathrm{C}(46 \mathrm{~A})$ & $123.0(3)$ \\
\hline $\mathrm{C}(16 \mathrm{~A})-\mathrm{C}(17 \mathrm{~A})-\mathrm{C}(46 \mathrm{~A})$ & $119.6(3)$ & $\mathrm{C}(17 \mathrm{~A})-\mathrm{C}(18 \mathrm{~A})-\mathrm{C}(19 \mathrm{~A})$ & $122.6(3)$ \\
\hline $\mathrm{C}(17 \mathrm{~A})-\mathrm{C}(18 \mathrm{~A})-\mathrm{H}(18 \mathrm{~A})$ & 118.7 & $\mathrm{C}(19 \mathrm{~A})-\mathrm{C}(18 \mathrm{~A})-\mathrm{H}(18 \mathrm{~A})$ & 118.7 \\
\hline$C(20 A)-C(19 A)-C(18 A)$ & $118.0(3)$ & $C(20 A)-C(19 A)-C(50 A)$ & $122.4(3)$ \\
\hline $\mathrm{C}(18 \mathrm{~A})-\mathrm{C}(19 \mathrm{~A})-\mathrm{C}(50 \mathrm{~A})$ & $119.6(3)$ & $\mathrm{C}(19 \mathrm{~A})-\mathrm{C}(20 \mathrm{~A})-\mathrm{C}(15 \mathrm{~A})$ & 121.1(3) \\
\hline C(19A)-C(20A)-H(20A) & 119.4 & $\mathrm{C}(15 \mathrm{~A})-\mathrm{C}(20 \mathrm{~A})-\mathrm{H}(20 \mathrm{~A})$ & 119.4 \\
\hline $\mathrm{C}(22 \mathrm{~A})-\mathrm{C}(21 \mathrm{~A})-\mathrm{C}(33 \mathrm{~A})$ & $107.1(3)$ & $\mathrm{C}(22 \mathrm{~A})-\mathrm{C}(21 \mathrm{~A})-\mathrm{C}(8 \mathrm{~A})$ & $126.2(3)$ \\
\hline$C(33 A)-C(21 A)-C(8 A)$ & $126.6(3)$ & $C(23 A)-C(22 A)-C(27 A)$ & $119.0(3)$ \\
\hline $\mathrm{C}(23 \mathrm{~A})-\mathrm{C}(22 \mathrm{~A})-\mathrm{C}(21 \mathrm{~A})$ & $131.4(3)$ & $\mathrm{C}(27 \mathrm{~A})-\mathrm{C}(22 \mathrm{~A})-\mathrm{C}(21 \mathrm{~A})$ & $109.5(3)$ \\
\hline$C(24 A)-C(23 A)-C(22 A)$ & $118.9(3)$ & $\mathrm{C}(24 \mathrm{~A})-\mathrm{C}(23 \mathrm{~A})-\mathrm{H}(23 \mathrm{~A})$ & 120.5 \\
\hline $\mathrm{C}(22 \mathrm{~A})-\mathrm{C}(23 \mathrm{~A})-\mathrm{H}(23 \mathrm{~A})$ & 120.5 & $\mathrm{C}(23 \mathrm{~A})-\mathrm{C}(24 \mathrm{~A})-\mathrm{C}(25 \mathrm{~A})$ & $122.2(3)$ \\
\hline $\mathrm{C}(23 \mathrm{~A})-\mathrm{C}(24 \mathrm{~A})-\mathrm{H}(24 \mathrm{~A})$ & 118.9 & $\mathrm{C}(25 \mathrm{~A})-\mathrm{C}(24 \mathrm{~A})-\mathrm{H}(24 \mathrm{~A})$ & 118.9 \\
\hline
\end{tabular}




\begin{tabular}{|c|c|c|c|}
\hline$C(26 A)-C(25 A)-C(24 A)$ & $118.6(3)$ & $\mathrm{C}(26 \mathrm{~A})-\mathrm{C}(25 \mathrm{~A})-\mathrm{C}(34 \mathrm{~A})$ & $119.9(3)$ \\
\hline $\mathrm{C}(24 \mathrm{~A})-\mathrm{C}(25 \mathrm{~A})-\mathrm{C}(34 \mathrm{~A})$ & $121.4(3)$ & $\mathrm{C}(27 \mathrm{~A})-\mathrm{C}(26 \mathrm{~A})-\mathrm{C}(25 \mathrm{~A})$ & $119.8(3)$ \\
\hline $\mathrm{C}(27 \mathrm{~A})-\mathrm{C}(26 \mathrm{~A})-\mathrm{H}(26 \mathrm{~A})$ & 120.1 & $\mathrm{C}(25 \mathrm{~A})-\mathrm{C}(26 \mathrm{~A})-\mathrm{H}(26 \mathrm{~A})$ & 120.1 \\
\hline $\mathrm{C}(26 \mathrm{~A})-\mathrm{C}(27 \mathrm{~A})-\mathrm{C}(22 \mathrm{~A})$ & $121.3(3)$ & $\mathrm{C}(26 \mathrm{~A})-\mathrm{C}(27 \mathrm{~A})-\mathrm{C}(28 \mathrm{~A})$ & $131.8(3)$ \\
\hline $\mathrm{C}(22 \mathrm{~A})-\mathrm{C}(27 \mathrm{~A})-\mathrm{C}(28 \mathrm{~A})$ & $106.8(3)$ & $\mathrm{C}(29 \mathrm{~A})-\mathrm{C}(28 \mathrm{~A})-\mathrm{C}(33 \mathrm{~A})$ & 121.1(3) \\
\hline $\mathrm{C}(29 \mathrm{~A})-\mathrm{C}(28 \mathrm{~A})-\mathrm{C}(27 \mathrm{~A})$ & $131.4(3)$ & $\mathrm{C}(33 \mathrm{~A})-\mathrm{C}(28 \mathrm{~A})-\mathrm{C}(27 \mathrm{~A})$ & $107.5(3)$ \\
\hline $\mathrm{C}(28 \mathrm{~A})-\mathrm{C}(29 \mathrm{~A})-\mathrm{C}(30 \mathrm{~A})$ & $119.2(3)$ & $\mathrm{C}(28 \mathrm{~A})-\mathrm{C}(29 \mathrm{~A})-\mathrm{H}(29 \mathrm{~A})$ & 120.4 \\
\hline $\mathrm{C}(30 \mathrm{~A})-\mathrm{C}(29 \mathrm{~A})-\mathrm{H}(29 \mathrm{~A})$ & 120.4 & $\mathrm{C}(31 \mathrm{~A})-\mathrm{C}(30 \mathrm{~A})-\mathrm{C}(29 \mathrm{~A})$ & $119.0(3)$ \\
\hline $\mathrm{C}(31 \mathrm{~A})-\mathrm{C}(30 \mathrm{~A})-\mathrm{C}(40 \mathrm{~A})$ & $120.8(3)$ & $\mathrm{C}(29 \mathrm{~A})-\mathrm{C}(30 \mathrm{~A})-\mathrm{C}(40 \mathrm{~A})$ & $120.3(3)$ \\
\hline$C(32 A)-C(31 A)-C(30 A)$ & $122.3(3)$ & $\mathrm{C}(32 \mathrm{~A})-\mathrm{C}(31 \mathrm{~A})-\mathrm{H}(31 \mathrm{~A})$ & 118.9 \\
\hline $\mathrm{C}(30 \mathrm{~A})-\mathrm{C}(31 \mathrm{~A})-\mathrm{H}(31 \mathrm{~A})$ & 118.9 & $\mathrm{C}(31 \mathrm{~A})-\mathrm{C}(32 \mathrm{~A})-\mathrm{C}(33 \mathrm{~A})$ & $119.0(3)$ \\
\hline $\mathrm{C}(31 \mathrm{~A})-\mathrm{C}(32 \mathrm{~A})-\mathrm{H}(32 \mathrm{~A})$ & 120.5 & $\mathrm{C}(33 \mathrm{~A})-\mathrm{C}(32 \mathrm{~A})-\mathrm{H}(32 \mathrm{~A})$ & 120.5 \\
\hline $\mathrm{C}(32 \mathrm{~A})-\mathrm{C}(33 \mathrm{~A})-\mathrm{C}(28 \mathrm{~A})$ & $119.4(3)$ & $C(32 \mathrm{~A})-\mathrm{C}(33 \mathrm{~A})-\mathrm{C}(21 \mathrm{~A})$ & $131.6(3)$ \\
\hline $\mathrm{C}(28 \mathrm{~A})-\mathrm{C}(33 \mathrm{~A})-\mathrm{C}(21 \mathrm{~A})$ & $109.0(3)$ & $\mathrm{C}(39 \mathrm{~A})-\mathrm{C}(34 \mathrm{~A})-\mathrm{C}(35 \mathrm{~A})$ & $116.6(4)$ \\
\hline $\mathrm{C}(39 \mathrm{~A})-\mathrm{C}(34 \mathrm{~A})-\mathrm{C}(25 \mathrm{~A})$ & $122.7(3)$ & $\mathrm{C}(35 \mathrm{~A})-\mathrm{C}(34 \mathrm{~A})-\mathrm{C}(25 \mathrm{~A})$ & $120.6(3)$ \\
\hline $\mathrm{C}(36 \mathrm{~A})-\mathrm{C}(35 \mathrm{~A})-\mathrm{C}(34 \mathrm{~A})$ & 121.3(4) & $\mathrm{C}(36 \mathrm{~A})-\mathrm{C}(35 \mathrm{~A})-\mathrm{H}(35 \mathrm{~A})$ & 119.4 \\
\hline $\mathrm{C}(34 \mathrm{~A})-\mathrm{C}(35 \mathrm{~A})-\mathrm{H}(35 \mathrm{~A})$ & 119.4 & $\mathrm{C}(37 \mathrm{~A})-\mathrm{C}(36 \mathrm{~A})-\mathrm{C}(35 \mathrm{~A})$ & $122.3(4)$ \\
\hline $\mathrm{C}(37 \mathrm{~A})-\mathrm{C}(36 \mathrm{~A})-\mathrm{H}(36 \mathrm{~A})$ & 118.9 & $\mathrm{C}(35 \mathrm{~A})-\mathrm{C}(36 \mathrm{~A})-\mathrm{H}(36 \mathrm{~A})$ & 118.9 \\
\hline$C(36 A)-C(37 A)-C(38 A)$ & $116.8(4)$ & $\mathrm{C}(36 \mathrm{~A})-\mathrm{C}(37 \mathrm{~A})-\mathrm{C}(54 \mathrm{~A})$ & $114.9(5)$ \\
\hline $\mathrm{C}(38 \mathrm{~A})-\mathrm{C}(37 \mathrm{~A})-\mathrm{C}(54 \mathrm{~A})$ & $128.2(5)$ & $\mathrm{C}(36 \mathrm{~A})-\mathrm{C}(37 \mathrm{~A})-\mathrm{C}\left(54^{\prime}\right)$ & $128.0(5)$ \\
\hline $\mathrm{C}(38 \mathrm{~A})-\mathrm{C}(37 \mathrm{~A})-\mathrm{C}\left(54^{\prime}\right)$ & $115.1(5)$ & $\mathrm{C}(39 \mathrm{~A})-\mathrm{C}(38 \mathrm{~A})-\mathrm{C}(37 \mathrm{~A})$ & $121.0(4)$ \\
\hline $\mathrm{C}(39 \mathrm{~A})-\mathrm{C}(38 \mathrm{~A})-\mathrm{H}(38 \mathrm{~A})$ & 119.5 & $\mathrm{C}(37 \mathrm{~A})-\mathrm{C}(38 \mathrm{~A})-\mathrm{H}(38 \mathrm{~A})$ & 119.5 \\
\hline $\mathrm{C}(34 \mathrm{~A})-\mathrm{C}(39 \mathrm{~A})-\mathrm{C}(38 \mathrm{~A})$ & $122.0(4)$ & $\mathrm{C}(34 \mathrm{~A})-\mathrm{C}(39 \mathrm{~A})-\mathrm{H}(39 \mathrm{~A})$ & 119.0 \\
\hline $\mathrm{C}(38 \mathrm{~A})-\mathrm{C}(39 \mathrm{~A})-\mathrm{H}(39 \mathrm{~A})$ & 119.0 & $\mathrm{C}(45 \mathrm{~A})-\mathrm{C}(40 \mathrm{~A})-\mathrm{C}(41 \mathrm{~A})$ & $117.3(3)$ \\
\hline$C(45 A)-C(40 A)-C(30 A)$ & $120.6(3)$ & $C(41 \mathrm{~A})-\mathrm{C}(40 \mathrm{~A})-\mathrm{C}(30 \mathrm{~A})$ & $122.0(3)$ \\
\hline $\mathrm{C}(42 \mathrm{~A})-\mathrm{C}(41 \mathrm{~A})-\mathrm{C}(40 \mathrm{~A})$ & $121.5(3)$ & $\mathrm{C}(42 \mathrm{~A})-\mathrm{C}(41 \mathrm{~A})-\mathrm{H}(41 \mathrm{~A})$ & 119.3 \\
\hline $\mathrm{C}(40 \mathrm{~A})-\mathrm{C}(41 \mathrm{~A})-\mathrm{H}(41 \mathrm{~A})$ & 119.3 & $\mathrm{C}(41 \mathrm{~A})-\mathrm{C}(42 \mathrm{~A})-\mathrm{C}(43 \mathrm{~A})$ & $121.6(3)$ \\
\hline $\mathrm{C}(41 \mathrm{~A})-\mathrm{C}(42 \mathrm{~A})-\mathrm{H}(42 \mathrm{~A})$ & 119.2 & $\mathrm{C}(43 \mathrm{~A})-\mathrm{C}(42 \mathrm{~A})-\mathrm{H}(42 \mathrm{~A})$ & 119.2 \\
\hline$C(42 A)-C(43 A)-C(44 A)$ & $116.3(3)$ & $\mathrm{C}(42 \mathrm{~A})-\mathrm{C}(43 \mathrm{~A})-\mathrm{C}(58 \mathrm{~A})$ & $123.9(4)$ \\
\hline $\mathrm{C}(44 \mathrm{~A})-\mathrm{C}(43 \mathrm{~A})-\mathrm{C}(58 \mathrm{~A})$ & $119.8(4)$ & $\mathrm{C}(42 \mathrm{~A})-\mathrm{C}(43 \mathrm{~A})-\mathrm{C}\left(58^{\prime}\right)$ & $121.5(6)$ \\
\hline$C(44 A)-C(43 A)-C\left(58^{\prime}\right)$ & $122.1(6)$ & $\mathrm{C}(45 \mathrm{~A})-\mathrm{C}(44 \mathrm{~A})-\mathrm{C}(43 \mathrm{~A})$ & $122.3(3)$ \\
\hline $\mathrm{C}(45 \mathrm{~A})-\mathrm{C}(44 \mathrm{~A})-\mathrm{H}(44 \mathrm{~A})$ & 118.8 & $\mathrm{C}(43 \mathrm{~A})-\mathrm{C}(44 \mathrm{~A})-\mathrm{H}(44 \mathrm{~A})$ & 118.8 \\
\hline $\mathrm{C}(44 \mathrm{~A})-\mathrm{C}(45 \mathrm{~A})-\mathrm{C}(40 \mathrm{~A})$ & $120.9(3)$ & $\mathrm{C}(44 \mathrm{~A})-\mathrm{C}(45 \mathrm{~A})-\mathrm{H}(45 \mathrm{~A})$ & 119.6 \\
\hline $\mathrm{C}(40 \mathrm{~A})-\mathrm{C}(45 \mathrm{~A})-\mathrm{H}(45 \mathrm{~A})$ & 119.6 & $C(49 A)-C(46 A)-C(17 A)$ & $112.7(3)$ \\
\hline $\mathrm{C}(49 \mathrm{~A})-\mathrm{C}(46 \mathrm{~A})-\mathrm{C}(48 \mathrm{~A})$ & $108.3(3)$ & $\mathrm{C}(17 \mathrm{~A})-\mathrm{C}(46 \mathrm{~A})-\mathrm{C}(48 \mathrm{~A})$ & $110.0(3)$ \\
\hline $\mathrm{C}(49 \mathrm{~A})-\mathrm{C}(46 \mathrm{~A})-\mathrm{C}(47 \mathrm{~A})$ & $107.5(3)$ & $\mathrm{C}(17 \mathrm{~A})-\mathrm{C}(46 \mathrm{~A})-\mathrm{C}(47 \mathrm{~A})$ & $108.4(3)$ \\
\hline
\end{tabular}




\begin{tabular}{|c|c|c|c|}
\hline $\mathrm{C}(48 \mathrm{~A})-\mathrm{C}(46 \mathrm{~A})-\mathrm{C}(47 \mathrm{~A})$ & $109.8(3)$ & $\mathrm{C}(46 \mathrm{~A})-\mathrm{C}(47 \mathrm{~A})-\mathrm{H}(47 \mathrm{~A})$ & 109.5 \\
\hline C(46A)-C(47A)-H(47B) & 109.5 & $\mathrm{H}(47 \mathrm{~A})-\mathrm{C}(47 \mathrm{~A})-\mathrm{H}(47 \mathrm{~B})$ & 109.5 \\
\hline $\mathrm{C}(46 \mathrm{~A})-\mathrm{C}(47 \mathrm{~A})-\mathrm{H}(47 \mathrm{C})$ & 109.5 & $\mathrm{H}(47 \mathrm{~A})-\mathrm{C}(47 \mathrm{~A})-\mathrm{H}(47 \mathrm{C})$ & 109.5 \\
\hline H(47B)-C(47A)-H(47C) & 109.5 & $\mathrm{C}(46 \mathrm{~A})-\mathrm{C}(48 \mathrm{~A})-\mathrm{H}(48 \mathrm{~A})$ & 109.5 \\
\hline C(46A)-C(48A)-H(48B) & 109.5 & $\mathrm{H}(48 \mathrm{~A})-\mathrm{C}(48 \mathrm{~A})-\mathrm{H}(48 \mathrm{~B})$ & 109.5 \\
\hline C(46A)-C(48A)-H(48C) & 109.5 & H(48A)-C(48A)-H(48C) & 109.5 \\
\hline H(48B)-C(48A)-H(48C) & 109.5 & $\mathrm{C}(46 \mathrm{~A})-\mathrm{C}(49 \mathrm{~A})-\mathrm{H}(49 \mathrm{~A})$ & 109.5 \\
\hline C(46A)-C(49A)-H(49B) & 109.5 & $\mathrm{H}(49 \mathrm{~A})-\mathrm{C}(49 \mathrm{~A})-\mathrm{H}(49 \mathrm{~B})$ & 109.5 \\
\hline C(46A)-C(49A)-H(49C) & 109.5 & $\mathrm{H}(49 \mathrm{~A})-\mathrm{C}(49 \mathrm{~A})-\mathrm{H}(49 \mathrm{C})$ & 109.5 \\
\hline H(49B)-C(49A)-H(49C) & 109.5 & $\mathrm{C}(51 \mathrm{~A})-\mathrm{C}(50 \mathrm{~A})-\mathrm{C}(19 \mathrm{~A})$ & $108.8(3)$ \\
\hline$C(51 A)-C(50 A)-C(53 A)$ & $107.8(3)$ & $C(19 A)-C(50 A)-C(53 A)$ & $112.1(3)$ \\
\hline$C(51 A)-C(50 A)-C(52 A)$ & $109.6(3)$ & $C(19 A)-C(50 A)-C(52 A)$ & $109.6(3)$ \\
\hline$C(53 A)-C(50 A)-C(52 A)$ & $109.0(3)$ & $\mathrm{C}(50 \mathrm{~A})-\mathrm{C}(51 \mathrm{~A})-\mathrm{H}(51 \mathrm{~A})$ & 109.5 \\
\hline $\mathrm{C}(50 \mathrm{~A})-\mathrm{C}(51 \mathrm{~A})-\mathrm{H}(51 \mathrm{~B})$ & 109.5 & $\mathrm{H}(51 \mathrm{~A})-\mathrm{C}(51 \mathrm{~A})-\mathrm{H}(51 \mathrm{~B})$ & 109.5 \\
\hline $\mathrm{C}(50 \mathrm{~A})-\mathrm{C}(51 \mathrm{~A})-\mathrm{H}(51 \mathrm{C})$ & 109.5 & $\mathrm{H}(51 \mathrm{~A})-\mathrm{C}(51 \mathrm{~A})-\mathrm{H}(51 \mathrm{C})$ & 109.5 \\
\hline $\mathrm{H}(51 \mathrm{~B})-\mathrm{C}(51 \mathrm{~A})-\mathrm{H}(51 \mathrm{C})$ & 109.5 & $\mathrm{C}(50 \mathrm{~A})-\mathrm{C}(52 \mathrm{~A})-\mathrm{H}(52 \mathrm{~A})$ & 109.5 \\
\hline $\mathrm{C}(50 \mathrm{~A})-\mathrm{C}(52 \mathrm{~A})-\mathrm{H}(52 \mathrm{~B})$ & 109.5 & $\mathrm{H}(52 \mathrm{~A})-\mathrm{C}(52 \mathrm{~A})-\mathrm{H}(52 \mathrm{~B})$ & 109.5 \\
\hline $\mathrm{C}(50 \mathrm{~A})-\mathrm{C}(52 \mathrm{~A})-\mathrm{H}(52 \mathrm{C})$ & 109.5 & $\mathrm{H}(52 \mathrm{~A})-\mathrm{C}(52 \mathrm{~A})-\mathrm{H}(52 \mathrm{C})$ & 109.5 \\
\hline $\mathrm{H}(52 \mathrm{~B})-\mathrm{C}(52 \mathrm{~A})-\mathrm{H}(52 \mathrm{C})$ & 109.5 & $\mathrm{C}(50 \mathrm{~A})-\mathrm{C}(53 \mathrm{~A})-\mathrm{H}(53 \mathrm{~A})$ & 109.5 \\
\hline $\mathrm{C}(50 \mathrm{~A})-\mathrm{C}(53 \mathrm{~A})-\mathrm{H}(53 \mathrm{~B})$ & 109.5 & $\mathrm{H}(53 \mathrm{~A})-\mathrm{C}(53 \mathrm{~A})-\mathrm{H}(53 \mathrm{~B})$ & 109.5 \\
\hline $\mathrm{C}(50 \mathrm{~A})-\mathrm{C}(53 \mathrm{~A})-\mathrm{H}(53 \mathrm{C})$ & 109.5 & $\mathrm{H}(53 \mathrm{~A})-\mathrm{C}(53 \mathrm{~A})-\mathrm{H}(53 \mathrm{C})$ & 109.5 \\
\hline $\mathrm{H}(53 \mathrm{~B})-\mathrm{C}(53 \mathrm{~A})-\mathrm{H}(53 \mathrm{C})$ & 109.5 & $\mathrm{C}(37 \mathrm{~A})-\mathrm{C}(54 \mathrm{~A})-\mathrm{C}(55 \mathrm{~A})$ & $110.8(7)$ \\
\hline $\mathrm{C}(37 \mathrm{~A})-\mathrm{C}(54 \mathrm{~A})-\mathrm{C}(57 \mathrm{~A})$ & $107.9(7)$ & $\mathrm{C}(55 \mathrm{~A})-\mathrm{C}(54 \mathrm{~A})-\mathrm{C}(57 \mathrm{~A})$ & $108.3(5)$ \\
\hline $\mathrm{C}(37 \mathrm{~A})-\mathrm{C}(54 \mathrm{~A})-\mathrm{C}(56 \mathrm{~A})$ & $111.0(7)$ & $\mathrm{C}(55 \mathrm{~A})-\mathrm{C}(54 \mathrm{~A})-\mathrm{C}(56 \mathrm{~A})$ & $109.5(5)$ \\
\hline$C(57 A)-C(54 A)-C(56 A)$ & $109.3(5)$ & $\mathrm{C}(54 \mathrm{~A})-\mathrm{C}(55 \mathrm{~A})-\mathrm{H}(55 \mathrm{~A})$ & 109.5 \\
\hline $\mathrm{C}(54 \mathrm{~A})-\mathrm{C}(55 \mathrm{~A})-\mathrm{H}(55 \mathrm{~B})$ & 109.5 & $\mathrm{H}(55 \mathrm{~A})-\mathrm{C}(55 \mathrm{~A})-\mathrm{H}(55 \mathrm{~B})$ & 109.5 \\
\hline $\mathrm{C}(54 \mathrm{~A})-\mathrm{C}(55 \mathrm{~A})-\mathrm{H}(55 \mathrm{C})$ & 109.5 & $\mathrm{H}(55 \mathrm{~A})-\mathrm{C}(55 \mathrm{~A})-\mathrm{H}(55 \mathrm{C})$ & 109.5 \\
\hline $\mathrm{H}(55 \mathrm{~B})-\mathrm{C}(55 \mathrm{~A})-\mathrm{H}(55 \mathrm{C})$ & 109.5 & $\mathrm{C}(54 \mathrm{~A})-\mathrm{C}(56 \mathrm{~A})-\mathrm{H}(56 \mathrm{~A})$ & 109.5 \\
\hline $\mathrm{C}(54 \mathrm{~A})-\mathrm{C}(56 \mathrm{~A})-\mathrm{H}(56 \mathrm{~B})$ & 109.5 & $\mathrm{H}(56 \mathrm{~A})-\mathrm{C}(56 \mathrm{~A})-\mathrm{H}(56 \mathrm{~B})$ & 109.5 \\
\hline $\mathrm{C}(54 \mathrm{~A})-\mathrm{C}(56 \mathrm{~A})-\mathrm{H}(56 \mathrm{C})$ & 109.5 & $\mathrm{H}(56 \mathrm{~A})-\mathrm{C}(56 \mathrm{~A})-\mathrm{H}(56 \mathrm{C})$ & 109.5 \\
\hline $\mathrm{H}(56 \mathrm{~B})-\mathrm{C}(56 \mathrm{~A})-\mathrm{H}(56 \mathrm{C})$ & 109.5 & $\mathrm{C}(54 \mathrm{~A})-\mathrm{C}(57 \mathrm{~A})-\mathrm{H}(57 \mathrm{~A})$ & 109.5 \\
\hline $\mathrm{C}(54 \mathrm{~A})-\mathrm{C}(57 \mathrm{~A})-\mathrm{H}(57 \mathrm{~B})$ & 109.5 & $\mathrm{H}(57 \mathrm{~A})-\mathrm{C}(57 \mathrm{~A})-\mathrm{H}(57 \mathrm{~B})$ & 109.5 \\
\hline $\mathrm{C}(54 \mathrm{~A})-\mathrm{C}(57 \mathrm{~A})-\mathrm{H}(57 \mathrm{C})$ & 109.5 & $\mathrm{H}(57 \mathrm{~A})-\mathrm{C}(57 \mathrm{~A})-\mathrm{H}(57 \mathrm{C})$ & 109.5 \\
\hline $\mathrm{H}(57 \mathrm{~B})-\mathrm{C}(57 \mathrm{~A})-\mathrm{H}(57 \mathrm{C})$ & 109.5 & $\mathrm{C}\left(55^{\prime}\right)-\mathrm{C}\left(54^{\prime}\right)-\mathrm{C}\left(57^{\prime}\right)$ & $107.6(5)$ \\
\hline $\mathrm{C}\left(55^{\prime}\right)-\mathrm{C}\left(54^{\prime}\right)-\mathrm{C}\left(56^{\prime}\right)$ & $108.7(5)$ & $\mathrm{C}\left(57^{\prime}\right)-\mathrm{C}\left(54^{\prime}\right)-\mathrm{C}\left(56^{\prime}\right)$ & $108.4(5)$ \\
\hline $\mathrm{C}\left(55^{\prime}\right)-\mathrm{C}\left(54^{\prime}\right)-\mathrm{C}(37 \mathrm{~A})$ & $109.4(7)$ & $\mathrm{C}\left(57^{\prime}\right)-\mathrm{C}\left(54^{\prime}\right)-\mathrm{C}(37 \mathrm{~A})$ & $113.7(7)$ \\
\hline
\end{tabular}




\begin{tabular}{|c|c|c|c|}
\hline $\mathrm{C}\left(56^{\prime}\right)-\mathrm{C}\left(54^{\prime}\right)-\mathrm{C}(37 \mathrm{~A})$ & $108.8(7)$ & $\mathrm{C}\left(54^{\prime}\right)-\mathrm{C}\left(55^{\prime}\right)-\mathrm{H}(55 \mathrm{D})$ & 109.5 \\
\hline $\mathrm{C}\left(54^{\prime}\right)-\mathrm{C}\left(55^{\prime}\right)-\mathrm{H}(55 \mathrm{E})$ & 109.5 & $\mathrm{H}(55 \mathrm{D})-\mathrm{C}\left(55^{\prime}\right)-\mathrm{H}(55 \mathrm{E})$ & 109.5 \\
\hline $\mathrm{C}\left(54^{\prime}\right)-\mathrm{C}\left(55^{\prime}\right)-\mathrm{H}(55 \mathrm{~F})$ & 109.5 & $\mathrm{H}(55 \mathrm{D})-\mathrm{C}\left(55^{\prime}\right)-\mathrm{H}(55 \mathrm{~F})$ & 109.5 \\
\hline $\mathrm{H}(55 \mathrm{E})-\mathrm{C}\left(55^{\prime}\right)-\mathrm{H}(55 \mathrm{~F})$ & 109.5 & $\mathrm{C}\left(54^{\prime}\right)-\mathrm{C}\left(56^{\prime}\right)-\mathrm{H}(56 \mathrm{D})$ & 109.5 \\
\hline $\mathrm{C}\left(54^{\prime}\right)-\mathrm{C}\left(56^{\prime}\right)-\mathrm{H}(56 \mathrm{E})$ & 109.5 & $\mathrm{H}(56 \mathrm{D})-\mathrm{C}\left(56^{\prime}\right)-\mathrm{H}(56 \mathrm{E})$ & 109.5 \\
\hline $\mathrm{C}\left(54^{\prime}\right)-\mathrm{C}\left(56^{\prime}\right)-\mathrm{H}(56 \mathrm{~F})$ & 109.5 & $\mathrm{H}(56 \mathrm{D})-\mathrm{C}\left(56^{\prime}\right)-\mathrm{H}(56 \mathrm{~F})$ & 109.5 \\
\hline $\mathrm{H}(56 \mathrm{E})-\mathrm{C}\left(56^{\prime}\right)-\mathrm{H}(56 \mathrm{~F})$ & 109.5 & $\mathrm{C}\left(54^{\prime}\right)-\mathrm{C}\left(57^{\prime}\right)-\mathrm{H}(57 \mathrm{D})$ & 109.5 \\
\hline $\mathrm{C}\left(54^{\prime}\right)-\mathrm{C}\left(57^{\prime}\right)-\mathrm{H}(57 \mathrm{E})$ & 109.5 & $\mathrm{H}(57 \mathrm{D})-\mathrm{C}\left(57^{\prime}\right)-\mathrm{H}(57 \mathrm{E})$ & 109.5 \\
\hline $\mathrm{C}\left(54^{\prime}\right)-\mathrm{C}\left(57^{\prime}\right)-\mathrm{H}(57 \mathrm{~F})$ & 109.5 & $\mathrm{H}(57 \mathrm{D})-\mathrm{C}\left(57^{\prime}\right)-\mathrm{H}(57 \mathrm{~F})$ & 109.5 \\
\hline $\mathrm{H}(57 \mathrm{E})-\mathrm{C}\left(57^{\prime}\right)-\mathrm{H}(57 \mathrm{~F})$ & 109.5 & $\mathrm{C}(43 \mathrm{~A})-\mathrm{C}(58 \mathrm{~A})-\mathrm{C}(59 \mathrm{~A})$ & $111.9(7)$ \\
\hline$C(43 A)-C(58 A)-C(60 A)$ & $108.6(6)$ & $\mathrm{C}(59 \mathrm{~A})-\mathrm{C}(58 \mathrm{~A})-\mathrm{C}(60 \mathrm{~A})$ & $109.6(4)$ \\
\hline$C(43 A)-C(58 A)-C(61 A)$ & $110.0(6)$ & $\mathrm{C}(59 \mathrm{~A})-\mathrm{C}(58 \mathrm{~A})-\mathrm{C}(61 \mathrm{~A})$ & $107.8(4)$ \\
\hline$C(60 A)-C(58 A)-C(61 A)$ & $108.9(4)$ & $\mathrm{C}(58 \mathrm{~A})-\mathrm{C}(59 \mathrm{~A})-\mathrm{H}(59 \mathrm{~A})$ & 109.5 \\
\hline $\mathrm{C}(58 \mathrm{~A})-\mathrm{C}(59 \mathrm{~A})-\mathrm{H}(59 \mathrm{~B})$ & 109.5 & $\mathrm{H}(59 \mathrm{~A})-\mathrm{C}(59 \mathrm{~A})-\mathrm{H}(59 \mathrm{~B})$ & 109.5 \\
\hline $\mathrm{C}(58 \mathrm{~A})-\mathrm{C}(59 \mathrm{~A})-\mathrm{H}(59 \mathrm{C})$ & 109.5 & $\mathrm{H}(59 \mathrm{~A})-\mathrm{C}(59 \mathrm{~A})-\mathrm{H}(59 \mathrm{C})$ & 109.5 \\
\hline $\mathrm{H}(59 \mathrm{~B})-\mathrm{C}(59 \mathrm{~A})-\mathrm{H}(59 \mathrm{C})$ & 109.5 & $\mathrm{C}(58 \mathrm{~A})-\mathrm{C}(60 \mathrm{~A})-\mathrm{H}(60 \mathrm{~A})$ & 109.5 \\
\hline $\mathrm{C}(58 \mathrm{~A})-\mathrm{C}(60 \mathrm{~A})-\mathrm{H}(60 \mathrm{~B})$ & 109.5 & $\mathrm{H}(60 \mathrm{~A})-\mathrm{C}(60 \mathrm{~A})-\mathrm{H}(60 \mathrm{~B})$ & 109.5 \\
\hline $\mathrm{C}(58 \mathrm{~A})-\mathrm{C}(60 \mathrm{~A})-\mathrm{H}(60 \mathrm{C})$ & 109.5 & $H(60 A)-C(60 A)-H(60 C)$ & 109.5 \\
\hline $\mathrm{H}(60 \mathrm{~B})-\mathrm{C}(60 \mathrm{~A})-\mathrm{H}(60 \mathrm{C})$ & 109.5 & $\mathrm{C}(58 \mathrm{~A})-\mathrm{C}(61 \mathrm{~A})-\mathrm{H}(61 \mathrm{~A})$ & 109.5 \\
\hline $\mathrm{C}(58 \mathrm{~A})-\mathrm{C}(61 \mathrm{~A})-\mathrm{H}(61 \mathrm{~B})$ & 109.5 & $H(61 A)-C(61 A)-H(61 B)$ & 109.5 \\
\hline $\mathrm{C}(58 \mathrm{~A})-\mathrm{C}(61 \mathrm{~A})-\mathrm{H}(61 \mathrm{C})$ & 109.5 & $H(61 A)-C(61 A)-H(61 C)$ & 109.5 \\
\hline$H(61 B)-C(61 A)-H(61 C)$ & 109.5 & $\mathrm{C}\left(59^{\prime}\right)-\mathrm{C}\left(58^{\prime}\right)-\mathrm{C}(43 \mathrm{~A})$ & $113.3(12)$ \\
\hline $\mathrm{C}\left(59^{\prime}\right)-\mathrm{C}\left(58^{\prime}\right)-\mathrm{C}\left(61^{\prime}\right)$ & $107.7(5)$ & $\mathrm{C}(43 \mathrm{~A})-\mathrm{C}\left(58^{\prime}\right)-\mathrm{C}\left(61^{\prime}\right)$ & $110.5(11)$ \\
\hline $\mathrm{C}\left(59^{\prime}\right)-\mathrm{C}\left(58^{\prime}\right)-\mathrm{C}\left(60^{\prime}\right)$ & $109.0(6)$ & $\mathrm{C}(43 \mathrm{~A})-\mathrm{C}\left(58^{\prime}\right)-\mathrm{C}\left(60^{\prime}\right)$ & $106.9(10)$ \\
\hline $\mathrm{C}\left(61^{\prime}\right)-\mathrm{C}\left(58^{\prime}\right)-\mathrm{C}\left(60^{\prime}\right)$ & $109.3(6)$ & $\mathrm{C}\left(58^{\prime}\right)-\mathrm{C}\left(59^{\prime}\right)-\mathrm{H}(59 \mathrm{D})$ & 109.5 \\
\hline $\mathrm{C}\left(58^{\prime}\right)-\mathrm{C}\left(59^{\prime}\right)-\mathrm{H}(59 \mathrm{E})$ & 109.5 & $\mathrm{H}(59 \mathrm{D})-\mathrm{C}\left(59^{\prime}\right)-\mathrm{H}(59 \mathrm{E})$ & 109.5 \\
\hline $\mathrm{C}\left(58^{\prime}\right)-\mathrm{C}\left(59^{\prime}\right)-\mathrm{H}(59 \mathrm{~F})$ & 109.5 & $\mathrm{H}(59 \mathrm{D})-\mathrm{C}\left(59^{\prime}\right)-\mathrm{H}(59 \mathrm{~F})$ & 109.5 \\
\hline $\mathrm{H}(59 \mathrm{E})-\mathrm{C}\left(59^{\prime}\right)-\mathrm{H}(59 \mathrm{~F})$ & 109.5 & $\mathrm{C}\left(58^{\prime}\right)-\mathrm{C}\left(60^{\prime}\right)-\mathrm{H}(60 \mathrm{D})$ & 109.5 \\
\hline $\mathrm{C}\left(58^{\prime}\right)-\mathrm{C}\left(60^{\prime}\right)-\mathrm{H}(60 \mathrm{E})$ & 109.5 & $\mathrm{H}(60 \mathrm{D})-\mathrm{C}\left(60^{\prime}\right)-\mathrm{H}(60 \mathrm{E})$ & 109.5 \\
\hline $\mathrm{C}\left(58^{\prime}\right)-\mathrm{C}\left(60^{\prime}\right)-\mathrm{H}(60 \mathrm{~F})$ & 109.5 & $\mathrm{H}(60 \mathrm{D})-\mathrm{C}\left(60^{\prime}\right)-\mathrm{H}(60 \mathrm{~F})$ & 109.5 \\
\hline $\mathrm{H}(60 \mathrm{E})-\mathrm{C}\left(60^{\prime}\right)-\mathrm{H}(60 \mathrm{~F})$ & 109.5 & $\mathrm{C}\left(58^{\prime}\right)-\mathrm{C}\left(61^{\prime}\right)-\mathrm{H}(61 \mathrm{D})$ & 109.5 \\
\hline $\mathrm{C}\left(58^{\prime}\right)-\mathrm{C}\left(61^{\prime}\right)-\mathrm{H}(61 \mathrm{E})$ & 109.5 & $\mathrm{H}(61 \mathrm{D})-\mathrm{C}\left(61^{\prime}\right)-\mathrm{H}(61 \mathrm{E})$ & 109.5 \\
\hline $\mathrm{C}\left(58^{\prime}\right)-\mathrm{C}\left(61^{\prime}\right)-\mathrm{H}(61 \mathrm{~F})$ & 109.5 & $\mathrm{H}(61 \mathrm{D})-\mathrm{C}\left(61^{\prime}\right)-\mathrm{H}(61 \mathrm{~F})$ & 109.5 \\
\hline $\mathrm{H}(61 \mathrm{E})-\mathrm{C}\left(61^{\prime}\right)-\mathrm{H}(61 \mathrm{~F})$ & 109.5 & & \\
\hline $\mathrm{C}(2 \mathrm{~B})-\mathrm{C}(1 \mathrm{~B})-\mathrm{C}(2 \mathrm{~B}) \# 1$ & $107.8(5)$ & $\mathrm{C}(2 \mathrm{~B})-\mathrm{C}(1 \mathrm{~B})-\mathrm{C}(14 \mathrm{~B})$ & $126.1(2)$ \\
\hline
\end{tabular}




\begin{tabular}{|c|c|c|c|}
\hline$C(2 B) \# 1-C(1 B)-C(14 B)$ & $126.1(2)$ & $\mathrm{C}(3 \mathrm{~B})-\mathrm{C}(2 \mathrm{~B})-\mathrm{C}(7 \mathrm{~B})$ & $118.8(3)$ \\
\hline $\mathrm{C}(3 \mathrm{~B})-\mathrm{C}(2 \mathrm{~B})-\mathrm{C}(1 \mathrm{~B})$ & $132.2(4)$ & $\mathrm{C}(7 \mathrm{~B})-\mathrm{C}(2 \mathrm{~B})-\mathrm{C}(1 \mathrm{~B})$ & $109.0(3)$ \\
\hline $\mathrm{C}(4 \mathrm{~B})-\mathrm{C}(3 \mathrm{~B})-\mathrm{C}(2 \mathrm{~B})$ & 119.4(4) & $\mathrm{C}(4 \mathrm{~B})-\mathrm{C}(3 \mathrm{~B})-\mathrm{H}(3 \mathrm{~B})$ & 120.3 \\
\hline $\mathrm{C}(2 \mathrm{~B})-\mathrm{C}(3 \mathrm{~B})-\mathrm{H}(3 \mathrm{~B})$ & 120.3 & $\mathrm{C}(3 \mathrm{~B})-\mathrm{C}(4 \mathrm{~B})-\mathrm{C}(5 \mathrm{~B})$ & $121.9(3)$ \\
\hline $\mathrm{C}(3 \mathrm{~B})-\mathrm{C}(4 \mathrm{~B})-\mathrm{H}(4 \mathrm{~B})$ & 119.1 & $\mathrm{C}(5 \mathrm{~B})-\mathrm{C}(4 \mathrm{~B})-\mathrm{H}(4 \mathrm{~B})$ & 119.1 \\
\hline $\mathrm{C}(6 \mathrm{~B})-\mathrm{C}(5 \mathrm{~B})-\mathrm{C}(4 \mathrm{~B})$ & $119.0(3)$ & $\mathrm{C}(6 \mathrm{~B})-\mathrm{C}(5 \mathrm{~B})-\mathrm{C}(8 \mathrm{~B})$ & $120.1(3)$ \\
\hline $\mathrm{C}(4 \mathrm{~B})-\mathrm{C}(5 \mathrm{~B})-\mathrm{C}(8 \mathrm{~B})$ & $120.9(3)$ & $\mathrm{C}(7 \mathrm{~B})-\mathrm{C}(6 \mathrm{~B})-\mathrm{C}(5 \mathrm{~B})$ & $119.5(3)$ \\
\hline $\mathrm{C}(7 \mathrm{~B})-\mathrm{C}(6 \mathrm{~B})-\mathrm{H}(6 \mathrm{~B})$ & 120.3 & $\mathrm{C}(5 \mathrm{~B})-\mathrm{C}(6 \mathrm{~B})-\mathrm{H}(6 \mathrm{~B})$ & 120.3 \\
\hline $\mathrm{C}(6 \mathrm{~B})-\mathrm{C}(7 \mathrm{~B})-\mathrm{C}(2 \mathrm{~B})$ & $121.4(3)$ & $\mathrm{C}(6 \mathrm{~B})-\mathrm{C}(7 \mathrm{~B})-\mathrm{C}(7 \mathrm{~B}) \# 1$ & $131.4(2)$ \\
\hline $\mathrm{C}(2 \mathrm{~B})-\mathrm{C}(7 \mathrm{~B})-\mathrm{C}(7 \mathrm{~B}) \# 1$ & $107.2(2)$ & $\mathrm{C}(13 \mathrm{~B})-\mathrm{C}(8 \mathrm{~B})-\mathrm{C}(9 \mathrm{~B})$ & $117.6(3)$ \\
\hline $\mathrm{C}(13 \mathrm{~B})-\mathrm{C}(8 \mathrm{~B})-\mathrm{C}(5 \mathrm{~B})$ & $121.6(3)$ & $\mathrm{C}(9 \mathrm{~B})-\mathrm{C}(8 \mathrm{~B})-\mathrm{C}(5 \mathrm{~B})$ & $120.8(3)$ \\
\hline $\mathrm{C}(10 \mathrm{~B})-\mathrm{C}(9 \mathrm{~B})-\mathrm{C}(8 \mathrm{~B})$ & $120.7(3)$ & $\mathrm{C}(10 \mathrm{~B})-\mathrm{C}(9 \mathrm{~B})-\mathrm{H}(9 \mathrm{~B})$ & 119.7 \\
\hline $\mathrm{C}(8 \mathrm{~B})-\mathrm{C}(9 \mathrm{~B})-\mathrm{H}(9 \mathrm{~B})$ & 119.7 & $\mathrm{C}(9 \mathrm{~B})-\mathrm{C}(10 \mathrm{~B})-\mathrm{C}(11 \mathrm{~B})$ & $122.1(3)$ \\
\hline C(9B)-C(10B)-H(10B) & 119.0 & $\mathrm{C}(11 \mathrm{~B})-\mathrm{C}(10 \mathrm{~B})-\mathrm{H}(10 \mathrm{~B})$ & 119.0 \\
\hline C(10B)-C(11B)-C(12B) & $117.1(3)$ & $\mathrm{C}(10 \mathrm{~B})-\mathrm{C}(11 \mathrm{~B})-\mathrm{C}(30)$ & $123.3(3)$ \\
\hline $\mathrm{C}(12 \mathrm{~B})-\mathrm{C}(11 \mathrm{~B})-\mathrm{C}(30)$ & $119.6(3)$ & $\mathrm{C}(13 \mathrm{~B})-\mathrm{C}(12 \mathrm{~B})-\mathrm{C}(11 \mathrm{~B})$ & $121.3(4)$ \\
\hline $\mathrm{C}(13 \mathrm{~B})-\mathrm{C}(12 \mathrm{~B})-\mathrm{H}(12 \mathrm{~B})$ & 119.3 & $\mathrm{C}(11 \mathrm{~B})-\mathrm{C}(12 \mathrm{~B})-\mathrm{H}(12 \mathrm{~B})$ & 119.3 \\
\hline $\mathrm{C}(12 \mathrm{~B})-\mathrm{C}(13 \mathrm{~B})-\mathrm{C}(8 \mathrm{~B})$ & $121.2(4)$ & $\mathrm{C}(12 \mathrm{~B})-\mathrm{C}(13 \mathrm{~B})-\mathrm{H}(13 \mathrm{~B})$ & 119.4 \\
\hline $\mathrm{C}(8 \mathrm{~B})-\mathrm{C}(13 \mathrm{~B})-\mathrm{H}(13 \mathrm{~B})$ & 119.4 & $\mathrm{C}(15 \mathrm{~B}) \# 1-\mathrm{C}(14 \mathrm{~B})-\mathrm{C}(15 \mathrm{~B})$ & $121.9(6)$ \\
\hline $\mathrm{C}(15 \mathrm{~B}) \# 1-\mathrm{C}(14 \mathrm{~B})-\mathrm{C}(1 \mathrm{~B})$ & $119.0(3)$ & $\mathrm{C}(15 \mathrm{~B})-\mathrm{C}(14 \mathrm{~B})-\mathrm{C}(1 \mathrm{~B})$ & $119.0(3)$ \\
\hline C(14B)-C(15B)-C(16B) & $118.9(5)$ & $C(14 B)-C(15 B)-C(20 B)$ & $121.6(4)$ \\
\hline C(16B)-C(15B)-C(20B) & $119.5(5)$ & $\mathrm{C}(21 \mathrm{~B})-\mathrm{C}(16 \mathrm{~B})-\mathrm{C}(15 \mathrm{~B})$ & $119.3(5)$ \\
\hline C(21B)-C(16B)-C(17B) & $124.9(5)$ & $\mathrm{C}(15 \mathrm{~B})-\mathrm{C}(16 \mathrm{~B})-\mathrm{C}(17 \mathrm{~B})$ & $115.8(5)$ \\
\hline C(18B)-C(17B)-C(16B) & $121.5(5)$ & $\mathrm{C}(18 \mathrm{~B})-\mathrm{C}(17 \mathrm{~B})-\mathrm{H}(17 \mathrm{~B})$ & 119.3 \\
\hline C(16B)-C(17B)-H(17B) & 119.3 & $\mathrm{C}(17 \mathrm{~B})-\mathrm{C}(18 \mathrm{~B})-\mathrm{C}(19 \mathrm{~B})$ & $122.0(6)$ \\
\hline C(17B)-C(18B)-H(18B) & 119.0 & $\mathrm{C}(19 \mathrm{~B})-\mathrm{C}(18 \mathrm{~B})-\mathrm{H}(18 \mathrm{~B})$ & 119.0 \\
\hline C(20B)-C(19B)-C(18B) & $119.2(6)$ & $\mathrm{C}(20 \mathrm{~B})-\mathrm{C}(19 \mathrm{~B})-\mathrm{H}(19 \mathrm{~B})$ & 120.4 \\
\hline C(18B)-C(19B)-H(19B) & 120.4 & $\mathrm{C}(19 \mathrm{~B})-\mathrm{C}(20 \mathrm{~B})-\mathrm{C}(15 \mathrm{~B})$ & $122.0(5)$ \\
\hline C(19B)-C(20B)-H(20B) & 119.0 & $\mathrm{C}(15 \mathrm{~B})-\mathrm{C}(20 \mathrm{~B})-\mathrm{H}(20 \mathrm{~B})$ & 119.0 \\
\hline $\mathrm{C}(16 \mathrm{~B}) \# 1-\mathrm{C}(21 \mathrm{~B})-\mathrm{C}(16 \mathrm{~B})$ & $121.5(6)$ & $\mathrm{C}(16 \mathrm{~B}) \# 1-\mathrm{C}(21 \mathrm{~B})-\mathrm{C}(22 \mathrm{~B})$ & $119.2(3)$ \\
\hline C(16B)-C(21B)-C(22B) & 119.2(3) & $\mathrm{C}(23 \mathrm{~B}) \# 1-\mathrm{C}(22 \mathrm{~B})-\mathrm{C}(23 \mathrm{~B})$ & $121.6(9)$ \\
\hline $\mathrm{C}(23 \mathrm{~B}) \# 1-\mathrm{C}(22 \mathrm{~B})-\mathrm{C}(21 \mathrm{~B})$ & $119.2(4)$ & $\mathrm{C}(23 \mathrm{~B})-\mathrm{C}(22 \mathrm{~B})-\mathrm{C}(21 \mathrm{~B})$ & $119.2(4)$ \\
\hline C(22B)-C(23B)-C(24B) & $121.5(8)$ & $\mathrm{C}(22 \mathrm{~B})-\mathrm{C}(23 \mathrm{~B})-\mathrm{H}(23 \mathrm{~B})$ & 119.3 \\
\hline $\mathrm{C}(24 \mathrm{~B})-\mathrm{C}(23 \mathrm{~B})-\mathrm{H}(23 \mathrm{~B})$ & 119.3 & $\mathrm{C}(23 \mathrm{~B})-\mathrm{C}(24 \mathrm{~B})-\mathrm{C}(25 \mathrm{~B})$ & $116.3(9)$ \\
\hline $\mathrm{C}(23 \mathrm{~B})-\mathrm{C}(24 \mathrm{~B})-\mathrm{C}\left(26^{\prime}\right)$ & $128.5(9)$ & $\mathrm{C}(25 \mathrm{~B})-\mathrm{C}(24 \mathrm{~B})-\mathrm{C}\left(26^{\prime}\right)$ & $115.1(9)$ \\
\hline C(23B)-C(24B)-C(26B) & 112.6(9) & $\mathrm{C}(25 \mathrm{~B})-\mathrm{C}(24 \mathrm{~B})-\mathrm{C}(26 \mathrm{~B})$ & $129.8(9)$ \\
\hline
\end{tabular}




\begin{tabular}{|c|c|c|c|}
\hline $\mathrm{C}(24 \mathrm{~B}) \# 1-\mathrm{C}(25 \mathrm{~B})-\mathrm{C}(24 \mathrm{~B})$ & $122.9(12)$ & $\mathrm{C}(24 \mathrm{~B}) \# 1-\mathrm{C}(25 \mathrm{~B})-\mathrm{H}(25 \mathrm{~B})$ & 118.5 \\
\hline $\mathrm{C}(24 \mathrm{~B})-\mathrm{C}(25 \mathrm{~B})-\mathrm{H}(25 \mathrm{~B})$ & 118.5 & $\mathrm{C}(27 \mathrm{~B})-\mathrm{C}(26 \mathrm{~B})-\mathrm{C}(29 \mathrm{~B})$ & $107.8(6)$ \\
\hline $\mathrm{C}(27 \mathrm{~B})-\mathrm{C}(26 \mathrm{~B})-\mathrm{C}(28 \mathrm{~B})$ & $110.2(6)$ & $\mathrm{C}(29 \mathrm{~B})-\mathrm{C}(26 \mathrm{~B})-\mathrm{C}(28 \mathrm{~B})$ & $109.4(6)$ \\
\hline $\mathrm{C}(27 \mathrm{~B})-\mathrm{C}(26 \mathrm{~B})-\mathrm{C}(24 \mathrm{~B})$ & $110.2(12)$ & $\mathrm{C}(29 \mathrm{~B})-\mathrm{C}(26 \mathrm{~B})-\mathrm{C}(24 \mathrm{~B})$ & $118.8(12)$ \\
\hline $\mathrm{C}(28 \mathrm{~B})-\mathrm{C}(26 \mathrm{~B})-\mathrm{C}(24 \mathrm{~B})$ & $100.2(10)$ & $\mathrm{C}(26 \mathrm{~B})-\mathrm{C}(27 \mathrm{~B})-\mathrm{H}(27 \mathrm{~A})$ & 109.5 \\
\hline $\mathrm{C}(26 \mathrm{~B})-\mathrm{C}(27 \mathrm{~B})-\mathrm{H}(27 \mathrm{~B})$ & 109.5 & $\mathrm{H}(27 \mathrm{~A})-\mathrm{C}(27 \mathrm{~B})-\mathrm{H}(27 \mathrm{~B})$ & 109.5 \\
\hline $\mathrm{C}(26 \mathrm{~B})-\mathrm{C}(27 \mathrm{~B})-\mathrm{H}(27 \mathrm{C})$ & 109.5 & $\mathrm{H}(27 \mathrm{~A})-\mathrm{C}(27 \mathrm{~B})-\mathrm{H}(27 \mathrm{C})$ & 109.5 \\
\hline H(27B)-C(27B)-H(27C) & 109.5 & $\mathrm{C}(26 \mathrm{~B})-\mathrm{C}(28 \mathrm{~B})-\mathrm{H}(28 \mathrm{~A})$ & 109.5 \\
\hline $\mathrm{C}(26 \mathrm{~B})-\mathrm{C}(28 \mathrm{~B})-\mathrm{H}(28 \mathrm{~B})$ & 109.5 & $\mathrm{H}(28 \mathrm{~A})-\mathrm{C}(28 \mathrm{~B})-\mathrm{H}(28 \mathrm{~B})$ & 109.5 \\
\hline $\mathrm{C}(26 \mathrm{~B})-\mathrm{C}(28 \mathrm{~B})-\mathrm{H}(28 \mathrm{C})$ & 109.5 & $\mathrm{H}(28 \mathrm{~A})-\mathrm{C}(28 \mathrm{~B})-\mathrm{H}(28 \mathrm{C})$ & 109.5 \\
\hline $\mathrm{H}(28 \mathrm{~B})-\mathrm{C}(28 \mathrm{~B})-\mathrm{H}(28 \mathrm{C})$ & 109.5 & $\mathrm{C}(26 \mathrm{~B})-\mathrm{C}(29 \mathrm{~B})-\mathrm{H}(29 \mathrm{~B})$ & 109.5 \\
\hline C(26B)-C(29B)-H(29C) & 109.5 & H(29B)-C(29B)-H(29C) & 109.5 \\
\hline$C(26 B)-C(29 B)-H(29 D)$ & 109.5 & H(29B)-C(29B)-H(29D) & 109.5 \\
\hline $\mathrm{H}(29 \mathrm{C})-\mathrm{C}(29 \mathrm{~B})-\mathrm{H}(29 \mathrm{D})$ & 109.5 & $\mathrm{C}(24 \mathrm{~B})-\mathrm{C}\left(26^{\prime}\right)-\mathrm{C}\left(27^{\prime}\right)$ & $131.6(12)$ \\
\hline $\mathrm{C}(24 \mathrm{~B})-\mathrm{C}\left(26^{\prime}\right)-\mathrm{C}\left(29^{\prime}\right)$ & $106.6(11)$ & $\mathrm{C}\left(27^{\prime}\right)-\mathrm{C}\left(26^{\prime}\right)-\mathrm{C}\left(29^{\prime}\right)$ & $108.2(6)$ \\
\hline $\mathrm{C}(24 \mathrm{~B})-\mathrm{C}\left(26^{\prime}\right)-\mathrm{C}\left(28^{\prime}\right)$ & $89.2(11)$ & $\mathrm{C}\left(27^{\prime}\right)-\mathrm{C}\left(26^{\prime}\right)-\mathrm{C}\left(28^{\prime}\right)$ & $109.1(6)$ \\
\hline $\mathrm{C}\left(29^{\prime}\right)-\mathrm{C}\left(26^{\prime}\right)-\mathrm{C}\left(28^{\prime}\right)$ & $109.6(6)$ & $\mathrm{C}\left(26^{\prime}\right)-\mathrm{C}\left(27^{\prime}\right)-\mathrm{H}(27 \mathrm{D})$ & 109.5 \\
\hline $\mathrm{C}\left(26^{\prime}\right)-\mathrm{C}\left(27^{\prime}\right)-\mathrm{H}(27 \mathrm{E})$ & 109.5 & $\mathrm{H}(27 \mathrm{D})-\mathrm{C}\left(27^{\prime}\right)-\mathrm{H}(27 \mathrm{E})$ & 109.5 \\
\hline $\mathrm{C}\left(26^{\prime}\right)-\mathrm{C}\left(27^{\prime}\right)-\mathrm{H}(27 \mathrm{~F})$ & 109.5 & $\mathrm{H}(27 \mathrm{D})-\mathrm{C}\left(27^{\prime}\right)-\mathrm{H}(27 \mathrm{~F})$ & 109.5 \\
\hline $\mathrm{H}(27 \mathrm{E})-\mathrm{C}\left(27^{\prime}\right)-\mathrm{H}(27 \mathrm{~F})$ & 109.5 & $\mathrm{C}\left(26^{\prime}\right)-\mathrm{C}\left(28^{\prime}\right)-\mathrm{H}(28 \mathrm{D})$ & 109.5 \\
\hline $\mathrm{C}\left(26^{\prime}\right)-\mathrm{C}\left(28^{\prime}\right)-\mathrm{H}(28 \mathrm{E})$ & 109.5 & $\mathrm{H}(28 \mathrm{D})-\mathrm{C}\left(28^{\prime}\right)-\mathrm{H}(28 \mathrm{E})$ & 109.5 \\
\hline $\mathrm{C}\left(26^{\prime}\right)-\mathrm{C}\left(28^{\prime}\right)-\mathrm{H}(28 \mathrm{~F})$ & 109.5 & $\mathrm{H}(28 \mathrm{D})-\mathrm{C}\left(28^{\prime}\right)-\mathrm{H}(28 \mathrm{~F})$ & 109.5 \\
\hline $\mathrm{H}(28 \mathrm{E})-\mathrm{C}\left(28^{\prime}\right)-\mathrm{H}(28 \mathrm{~F})$ & 109.5 & $\mathrm{C}\left(26^{\prime}\right)-\mathrm{C}\left(29^{\prime}\right)-\mathrm{H}(29 \mathrm{E})$ & 109.5 \\
\hline $\mathrm{C}\left(26^{\prime}\right)-\mathrm{C}\left(29^{\prime}\right)-\mathrm{H}(29 \mathrm{~F})$ & 109.5 & $\mathrm{H}(29 \mathrm{E})-\mathrm{C}\left(29^{\prime}\right)-\mathrm{H}(29 \mathrm{~F})$ & 109.5 \\
\hline $\mathrm{C}\left(26^{\prime}\right)-\mathrm{C}\left(29^{\prime}\right)-\mathrm{H}(29 \mathrm{G})$ & 109.5 & $\mathrm{H}(29 \mathrm{E})-\mathrm{C}\left(29^{\prime}\right)-\mathrm{H}(29 \mathrm{G})$ & 109.5 \\
\hline H(29F)-C(29')-H(29G) & 109.5 & $\mathrm{C}(33)-\mathrm{C}(30)-\mathrm{C}(32)$ & $108.9(4)$ \\
\hline $\mathrm{C}(33)-\mathrm{C}(30)-\mathrm{C}(11 \mathrm{~B})$ & $109.6(3)$ & $\mathrm{C}(32)-\mathrm{C}(30)-\mathrm{C}(11 \mathrm{~B})$ & $108.8(3)$ \\
\hline$C(33)-C(30)-C(31)$ & $108.7(4)$ & $C(32)-C(30)-C(31)$ & $108.8(3)$ \\
\hline$C(11 B)-C(30)-C(31)$ & $112.0(3)$ & $\mathrm{C}(30)-\mathrm{C}(31)-\mathrm{H}(31 \mathrm{~B})$ & 109.5 \\
\hline $\mathrm{C}(30)-\mathrm{C}(31)-\mathrm{H}(31 \mathrm{C})$ & 109.5 & $\mathrm{H}(31 \mathrm{~B})-\mathrm{C}(31)-\mathrm{H}(31 \mathrm{C})$ & 109.5 \\
\hline $\mathrm{C}(30)-\mathrm{C}(31)-\mathrm{H}(31 \mathrm{D})$ & 109.5 & $\mathrm{H}(31 \mathrm{~B})-\mathrm{C}(31)-\mathrm{H}(31 \mathrm{D})$ & 109.5 \\
\hline $\mathrm{H}(31 \mathrm{C})-\mathrm{C}(31)-\mathrm{H}(31 \mathrm{D})$ & 109.5 & $\mathrm{C}(30)-\mathrm{C}(32)-\mathrm{H}(32 \mathrm{~B})$ & 109.5 \\
\hline $\mathrm{C}(30)-\mathrm{C}(32)-\mathrm{H}(32 \mathrm{C})$ & 109.5 & $\mathrm{H}(32 \mathrm{~B})-\mathrm{C}(32)-\mathrm{H}(32 \mathrm{C})$ & 109.5 \\
\hline $\mathrm{C}(30)-\mathrm{C}(32)-\mathrm{H}(32 \mathrm{D})$ & 109.5 & $\mathrm{H}(32 \mathrm{~B})-\mathrm{C}(32)-\mathrm{H}(32 \mathrm{D})$ & 109.5 \\
\hline $\mathrm{H}(32 \mathrm{C})-\mathrm{C}(32)-\mathrm{H}(32 \mathrm{D})$ & 109.5 & $\mathrm{C}(30)-\mathrm{C}(33)-\mathrm{H}(33 \mathrm{~A})$ & 109.5 \\
\hline $\mathrm{C}(30)-\mathrm{C}(33)-\mathrm{H}(33 \mathrm{~B})$ & 109.5 & $\mathrm{H}(33 \mathrm{~A})-\mathrm{C}(33)-\mathrm{H}(33 \mathrm{~B})$ & 109.5 \\
\hline
\end{tabular}




$\begin{array}{llll}\mathrm{C}(30)-\mathrm{C}(33)-\mathrm{H}(33 \mathrm{C}) & 109.5 & \mathrm{H}(33 \mathrm{~A})-\mathrm{C}(33)-\mathrm{H}(33 \mathrm{C}) & 109.5 \\ \mathrm{H}(33 \mathrm{~B})-\mathrm{C}(33)-\mathrm{H}(33 \mathrm{C}) & 109.5 & \end{array}$

Symmetry transformations used to generate equivalent atoms: \#1 - $\mathrm{x}+1, \mathrm{y},-\mathrm{z}+3 / 2$

Table S6. Anisotropic displacement parameters $\left(\AA^{2} \times 10^{3}\right)$ for FR-1. The anisotropic displacement factor exponent takes the form: $-2 \pi^{2}\left[h^{2} a^{* 2} U^{11}+\ldots+2 h \mathbf{k} a^{*} b^{*} U^{12}\right]$

\begin{tabular}{|c|c|c|c|c|c|c|}
\hline & $\mathrm{U}^{11}$ & $\mathrm{U}^{22}$ & $\mathrm{U}^{33}$ & $\mathrm{U}^{23}$ & $\mathrm{U}^{13}$ & $\mathrm{U}^{12}$ \\
\hline$C(1 \mathrm{~A})$ & $24(2)$ & $20(2)$ & $19(2)$ & $1(1)$ & $0(1)$ & $-5(1)$ \\
\hline$C(2 \mathrm{~A})$ & $25(2)$ & $21(2)$ & $22(2)$ & $0(1)$ & $3(1)$ & $-7(1)$ \\
\hline$C(3 \mathrm{~A})$ & $30(2)$ & $25(2)$ & $27(2)$ & $-3(1)$ & $7(2)$ & $-2(1)$ \\
\hline$C(4 A)$ & $32(2)$ & $24(2)$ & $36(2)$ & $-6(2)$ & $9(2)$ & $-1(2)$ \\
\hline$C(5 \mathrm{~A})$ & $28(2)$ & $23(2)$ & $38(2)$ & $0(2)$ & $1(2)$ & $3(1)$ \\
\hline$C(6 A)$ & $31(2)$ & $23(2)$ & $25(2)$ & $5(1)$ & $2(2)$ & $0(1)$ \\
\hline$C(7 A)$ & $21(2)$ & $22(2)$ & $24(2)$ & $1(1)$ & $2(1)$ & $-4(1)$ \\
\hline $\mathrm{C}(8 \mathrm{~A})$ & $25(2)$ & $22(2)$ & $21(2)$ & $2(1)$ & $2(1)$ & $-4(1)$ \\
\hline $\mathrm{C}(9 \mathrm{~A})$ & $24(2)$ & $22(2)$ & $21(2)$ & $0(1)$ & $2(1)$ & $-5(1)$ \\
\hline$C(10 A)$ & $29(2)$ & $27(2)$ & $24(2)$ & $-1(1)$ & $7(1)$ & $0(1)$ \\
\hline$C(11 \mathrm{~A})$ & $29(2)$ & $33(2)$ & $30(2)$ & $-5(2)$ & $4(2)$ & $2(2)$ \\
\hline $\mathrm{C}(12 \mathrm{~A})$ & $28(2)$ & $26(2)$ & $35(2)$ & $-1(2)$ & $-1(2)$ & $5(1)$ \\
\hline$C(13 A)$ & $29(2)$ & $27(2)$ & $24(2)$ & $2(1)$ & $-3(2)$ & $-1(1)$ \\
\hline$C(14 A)$ & $22(2)$ & $20(2)$ & $22(2)$ & $0(1)$ & $-1(1)$ & $-5(1)$ \\
\hline$C(15 A)$ & $29(2)$ & $23(2)$ & $23(2)$ & $2(1)$ & $6(1)$ & $2(1)$ \\
\hline$C(16 A)$ & $28(2)$ & $22(2)$ & $24(2)$ & $0(1)$ & $3(1)$ & $-2(1)$ \\
\hline$C(17 \mathrm{~A})$ & $31(2)$ & $25(2)$ & $23(2)$ & $1(1)$ & $6(2)$ & $3(1)$ \\
\hline $\mathrm{C}(18 \mathrm{~A})$ & $42(2)$ & $27(2)$ & $20(2)$ & $5(1)$ & $4(2)$ & $0(2)$ \\
\hline$C(19 A)$ & $41(2)$ & $26(2)$ & $27(2)$ & $4(1)$ & $5(2)$ & $-4(2)$ \\
\hline$C(20 A)$ & $36(2)$ & $24(2)$ & $24(2)$ & $-1(1)$ & $1(2)$ & $-2(2)$ \\
\hline$C(21 \mathrm{~A})$ & $26(2)$ & $22(2)$ & $22(2)$ & $1(1)$ & $3(1)$ & $4(1)$ \\
\hline$C(22 \mathrm{~A})$ & $26(2)$ & $26(2)$ & $27(2)$ & $2(1)$ & $7(2)$ & $4(1)$ \\
\hline & $\mathrm{U}^{11}$ & $\mathrm{U}^{22}$ & $\mathrm{U}^{33}$ & $\mathrm{U}^{23}$ & $\mathrm{U}^{13}$ & $\mathrm{U}^{12}$ \\
\hline
\end{tabular}




\begin{tabular}{|c|c|c|c|c|c|c|}
\hline$C(23 \mathrm{~A})$ & $29(2)$ & $32(2)$ & $28(2)$ & $3(2)$ & $6(2)$ & $0(2)$ \\
\hline$C(24 \mathrm{~A})$ & $25(2)$ & $31(2)$ & $38(2)$ & $3(2)$ & $3(2)$ & $-1(2)$ \\
\hline$C(25 \mathrm{~A})$ & $27(2)$ & $24(2)$ & $32(2)$ & $0(1)$ & 2(2) & $3(1)$ \\
\hline$C(26 \mathrm{~A})$ & $26(2)$ & $26(2)$ & $22(2)$ & $3(1)$ & $4(1)$ & $3(1)$ \\
\hline $\mathrm{C}(27 \mathrm{~A})$ & $29(2)$ & $20(2)$ & $23(2)$ & $2(1)$ & $5(1)$ & $4(1)$ \\
\hline $\mathrm{C}(28 \mathrm{~A})$ & $25(2)$ & 21(2) & $22(2)$ & $2(1)$ & $5(1)$ & $5(1)$ \\
\hline$C(29 A)$ & $27(2)$ & $22(2)$ & $21(2)$ & $3(1)$ & $5(1)$ & $5(1)$ \\
\hline $\mathrm{C}(30 \mathrm{~A})$ & $27(2)$ & $21(2)$ & $24(2)$ & $3(1)$ & $5(1)$ & $4(1)$ \\
\hline $\mathrm{C}(31 \mathrm{~A})$ & $26(2)$ & $25(2)$ & $27(2)$ & $0(1)$ & $2(1)$ & 2(1) \\
\hline $\mathrm{C}(32 \mathrm{~A})$ & $28(2)$ & $27(2)$ & $19(2)$ & $4(1)$ & $0(1)$ & $3(1)$ \\
\hline $\mathrm{C}(33 \mathrm{~A})$ & $29(2)$ & $19(2)$ & $22(2)$ & $0(1)$ & $4(1)$ & $5(1)$ \\
\hline $\mathrm{C}(34 \mathrm{~A})$ & $30(2)$ & $24(2)$ & $38(2)$ & $3(2)$ & $0(2)$ & $-1(2)$ \\
\hline $\mathrm{C}(35 \mathrm{~A})$ & $31(2)$ & $48(2)$ & $46(2)$ & $3(2)$ & $-2(2)$ & $0(2)$ \\
\hline$C(36 \mathrm{~A})$ & $37(2)$ & $55(3)$ & $57(3)$ & $-2(2)$ & $-12(2)$ & $4(2)$ \\
\hline $\mathrm{C}(37 \mathrm{~A})$ & $48(3)$ & $40(2)$ & $53(3)$ & $-1(2)$ & $-17(2)$ & $-2(2)$ \\
\hline $\mathrm{C}(38 \mathrm{~A})$ & $55(3)$ & $43(2)$ & $34(2)$ & $-1(2)$ & $-8(2)$ & $-3(2)$ \\
\hline$C(39 A)$ & $37(2)$ & $37(2)$ & $34(2)$ & $4(2)$ & $-7(2)$ & $0(2)$ \\
\hline $\mathrm{C}(40 \mathrm{~A})$ & $26(2)$ & $23(2)$ & $26(2)$ & $2(1)$ & $5(1)$ & 2(1) \\
\hline $\mathrm{C}(41 \mathrm{~A})$ & $26(2)$ & $30(2)$ & $25(2)$ & $5(1)$ & $5(1)$ & $4(1)$ \\
\hline $\mathrm{C}(42 \mathrm{~A})$ & $30(2)$ & $29(2)$ & $26(2)$ & $4(1)$ & $0(2)$ & 1(2) \\
\hline $\mathrm{C}(43 \mathrm{~A})$ & $31(2)$ & $25(2)$ & $22(2)$ & $0(1)$ & $0(1)$ & $-1(1)$ \\
\hline $\mathrm{C}(44 \mathrm{~A})$ & $25(2)$ & $36(2)$ & $39(2)$ & $6(2)$ & $7(2)$ & 4(2) \\
\hline $\mathrm{C}(45 \mathrm{~A})$ & $30(2)$ & $32(2)$ & $37(2)$ & $11(2)$ & $3(2)$ & $6(2)$ \\
\hline $\mathrm{C}(46 \mathrm{~A})$ & $34(2)$ & $30(2)$ & $24(2)$ & $-1(1)$ & $2(2)$ & 1(2) \\
\hline $\mathrm{C}(47 \mathrm{~A})$ & $48(2)$ & $31(2)$ & $30(2)$ & $-4(2)$ & $2(2)$ & $0(2)$ \\
\hline $\mathrm{C}(48 \mathrm{~A})$ & $36(2)$ & $42(2)$ & $30(2)$ & $1(2)$ & $-3(2)$ & $-3(2)$ \\
\hline $\mathrm{C}(49 \mathrm{~A})$ & $44(2)$ & $42(2)$ & $24(2)$ & $1(2)$ & $1(2)$ & $-3(2)$ \\
\hline $\mathrm{C}(50 \mathrm{~A})$ & $53(2)$ & $32(2)$ & $31(2)$ & $3(2)$ & $5(2)$ & $-12(2)$ \\
\hline $\mathrm{C}(51 \mathrm{~A})$ & $66(3)$ & $32(2)$ & $55(3)$ & $8(2)$ & $3(2)$ & $-7(2)$ \\
\hline $\mathrm{C}(52 \mathrm{~A})$ & $60(3)$ & $42(3)$ & $67(3)$ & $-1(2)$ & $26(2)$ & $-16(2)$ \\
\hline $\mathrm{C}(53 \mathrm{~A})$ & $75(3)$ & 41(3) & $52(3)$ & $4(2)$ & $0(2)$ & $-26(2)$ \\
\hline \multirow[t]{2}{*}{$\mathrm{C}(54 \mathrm{~A})$} & $68(3)$ & $78(3)$ & $53(3)$ & $-6(3)$ & $-27(2)$ & $3(2)$ \\
\hline & $\mathrm{U}^{11}$ & $\mathrm{U}^{22}$ & $\mathrm{U}^{33}$ & $\mathrm{U}^{23}$ & $\mathrm{U}^{13}$ & $\mathrm{U}^{12}$ \\
\hline
\end{tabular}




\begin{tabular}{|c|c|c|c|c|c|c|}
\hline$C(55 A)$ & $71(4)$ & $80(3)$ & $58(4)$ & $-12(3)$ & $-29(3)$ & $-1(3)$ \\
\hline$C(56 A)$ & $61(3)$ & $80(4)$ & $54(4)$ & $0(3)$ & $-29(3)$ & $3(3)$ \\
\hline$C(57 A)$ & $71(3)$ & $85(4)$ & $55(3)$ & $-9(3)$ & $-26(3)$ & $3(3)$ \\
\hline $\mathrm{C}\left(54^{\prime}\right)$ & $68(3)$ & $78(3)$ & $53(3)$ & $-6(3)$ & $-28(2)$ & $3(2)$ \\
\hline$C\left(55^{\prime}\right)$ & $78(4)$ & $83(3)$ & $61(4)$ & $-11(3)$ & $-31(3)$ & $0(3)$ \\
\hline$C\left(56^{\prime}\right)$ & $63(3)$ & $83(4)$ & $53(4)$ & $0(3)$ & $-30(3)$ & $1(3)$ \\
\hline$C\left(57^{\prime}\right)$ & $68(4)$ & $82(4)$ & $52(3)$ & $-3(3)$ & $-26(3)$ & $9(3)$ \\
\hline $\mathrm{C}(58 \mathrm{~A})$ & $18(3)$ & $14(3)$ & $13(3)$ & $0(2)$ & $4(3)$ & $0(3)$ \\
\hline$C(59 A)$ & $30(4)$ & $22(4)$ & $13(3)$ & $9(3)$ & $2(3)$ & $-3(3)$ \\
\hline$C(60 A)$ & $33(4)$ & $31(4)$ & $20(3)$ & $-10(3)$ & $9(3)$ & $-3(3)$ \\
\hline$C(61 \mathrm{~A})$ & $19(3)$ & $17(4)$ & $24(3)$ & $-6(3)$ & $2(3)$ & $1(3)$ \\
\hline $\mathrm{C}\left(58^{\prime}\right)$ & $69(6)$ & $60(6)$ & $72(7)$ & $1(5)$ & $30(5)$ & $-14(5)$ \\
\hline$C\left(59^{\prime}\right)$ & $74(7)$ & $60(6)$ & $73(8)$ & $2(5)$ & $27(6)$ & $-15(5)$ \\
\hline$C\left(60^{\prime}\right)$ & $78(7)$ & $66(7)$ & $77(7)$ & $-3(5)$ & $35(6)$ & $-13(6)$ \\
\hline \multirow[t]{2}{*}{$C\left(61^{\prime}\right)$} & $73(7)$ & $68(7)$ & $79(7)$ & $6(5)$ & $24(6)$ & $-16(5)$ \\
\hline & $\mathrm{U}^{11}$ & $\mathrm{U}^{22}$ & $\mathrm{U}^{33}$ & $\mathrm{U}^{23}$ & $\mathrm{U}^{13}$ & $\mathrm{U}^{12}$ \\
\hline$C(1 B)$ & $25(2)$ & $41(3)$ & $24(2)$ & 0 & $-5(2)$ & 0 \\
\hline$C(2 B)$ & $25(2)$ & $36(2)$ & $27(2)$ & $1(2)$ & $-5(2)$ & $3(2)$ \\
\hline$C(3 B)$ & $34(2)$ & $33(2)$ & $42(2)$ & $1(2)$ & $7(2)$ & $6(2)$ \\
\hline$C(4 B)$ & $31(2)$ & $37(2)$ & $45(2)$ & $3(2)$ & $12(2)$ & $6(2)$ \\
\hline$C(5 B)$ & $29(2)$ & $38(2)$ & $32(2)$ & $2(2)$ & $3(2)$ & $2(2)$ \\
\hline$C(6 B)$ & $29(2)$ & $35(2)$ & $28(2)$ & $2(2)$ & $2(2)$ & $5(2)$ \\
\hline$C(7 B)$ & $25(2)$ & $37(2)$ & $22(2)$ & $1(1)$ & $-3(1)$ & $2(2)$ \\
\hline $\mathrm{C}(8 \mathrm{~B})$ & $29(2)$ & $35(2)$ & $35(2)$ & $5(2)$ & $7(2)$ & $3(2)$ \\
\hline C(9B) & $33(2)$ & $33(2)$ & $39(2)$ & $5(2)$ & $7(2)$ & $4(2)$ \\
\hline$C(10 B)$ & $31(2)$ & $34(2)$ & $35(2)$ & $7(2)$ & $9(2)$ & $3(2)$ \\
\hline$C(11 B)$ & $32(2)$ & $34(2)$ & $32(2)$ & $5(2)$ & $5(2)$ & $2(2)$ \\
\hline$C(12 B)$ & $36(2)$ & $36(2)$ & $48(2)$ & $9(2)$ & $9(2)$ & $3(2)$ \\
\hline \multirow[t]{2}{*}{$C(13 B)$} & $27(2)$ & $44(2)$ & $48(2)$ & $6(2)$ & $6(2)$ & $4(2)$ \\
\hline & $\mathrm{U}^{11}$ & $\mathrm{U}^{22}$ & $\mathrm{U}^{33}$ & $\mathrm{U}^{23}$ & $\mathrm{U}^{13}$ & $\mathrm{U}^{12}$ \\
\hline$C(14 B)$ & $26(3)$ & $36(3)$ & $42(3)$ & 0 & $6(2)$ & 0 \\
\hline
\end{tabular}




$\begin{array}{llllccc}\mathrm{C}(15 \mathrm{~B}) & 37(2) & 40(2) & 70(3) & 12(2) & 19(2) & 4(2) \\ \mathrm{C}(16 \mathrm{~B}) & 38(2) & 48(3) & 106(4) & 26(3) & 23(3) & 6(2) \\ \mathrm{C}(17 \mathrm{~B}) & 63(3) & 54(3) & 123(5) & 38(4) & 44(4) & 7(3) \\ \mathrm{C}(18 \mathrm{~B}) & 61(3) & 89(5) & 90(4) & 44(4) & 22(3) & 19(3) \\ \mathrm{C}(19 \mathrm{~B}) & 56(3) & 86(4) & 63(3) & 29(3) & 5(3) & 8(3) \\ \mathrm{C}(20 \mathrm{~B}) & 46(3) & 69(3) & 53(3) & 22(2) & 5(2) & 7(2) \\ \mathrm{C}(21 \mathrm{~B}) & 52(4) & 38(4) & 138(8) & 0 & 50(5) & 0 \\ \mathrm{C}(22 \mathrm{~B}) & 54(5) & 41(4) & 230(13) & 0 & 58(6) & 0 \\ \mathrm{C}(23 \mathrm{~B}) & 64(4) & 69(4) & 214(9) & 51(5) & 13(5) & -4(3) \\ \mathrm{C}(24 \mathrm{~B}) & 85(3) & 81(3) & 304(8) & 17(3) & -6(4) & -4(2) \\ \mathrm{C}(25 \mathrm{~B}) & 87(3) & 81(4) & 306(8) & 0 & -10(5) & 0 \\ \mathrm{C}(26 \mathrm{~B}) & 88(3) & 85(4) & 302(8) & 20(3) & -6(4) & -6(2) \\ \mathrm{C}(27 \mathrm{~B}) & 84(4) & 88(5) & 301(8) & 22(4) & -4(5) & -7(4) \\ \mathrm{C}(28 \mathrm{~B}) & 92(4) & 84(4) & 302(8) & 23(4) & -5(5) & -8(3) \\ \mathrm{C}(29 B) & 99(5) & 93(5) & 302(8) & 15(5) & 0(5) & -13(4) \\ \mathrm{C}\left(26{ }^{\prime}\right) & 88(3) & 84(4) & 303(8) & 21(3) & -6(4) & -7(2) \\ \mathrm{C}\left(27^{\prime}\right) & 92(4) & 85(4) & 304(8) & 21(4) & -4(5) & -11(3) \\ \mathrm{C}\left(28^{\prime}\right) & 96(4) & 92(4) & 303(8) & 22(4) & -8(5) & -9(3) \\ \mathrm{C}\left(29{ }^{\prime}\right) & 82(4) & 86(5) & 300(8) & 20(4) & -4(5) & -10(3) \\ \mathrm{C}(30) & 33(2) & 37(2) & 41(2) & 8(2) & 4(2) & 0(2) \\ \mathrm{C}(31) & 38(2) & 54(3) & 61(3) & 18(2) & 5(2) & -10(2) \\ \mathrm{C}(32) & 43(2) & 67(3) & 51(3) & 24(2) & 5(2) & -5(2) \\ \mathrm{C}(33) & 65(3) & 43(3) & 62(3) & 5(2) & 11(2) & -13(2) \\ & & & & & \\ \end{array}$

Table S7. Hydrogen coordinates ( $\left.\times 10^{4}\right)$ and isotropic displacement parameters $\left(\AA^{2} \times 10^{3}\right)$ for FR-1.

\begin{tabular}{lcccc}
\hline & $x$ & $y$ & $z$ & $U(e q)$ \\
& & & & \\
$H(3 A)$ & 3513 & 6040 & 1684 & 33 \\
$H(4 A)$ & 3798 & 5147 & 2234 & 36 \\
$H(5 A)$ & 3798 & 4808 & 3606 & 36 \\
$H(6 A)$ & 3516 & 5374 & 4420 & 32 \\
$H(10 A)$ & 2832 & 7279 & 4795 & 31 \\
$H(11 A)$ & 2573 & 8225 & 4279 & 36 \\
$H(12 A)$ & 2578 & 8566 & 2922 & 36
\end{tabular}




\begin{tabular}{|c|c|c|c|c|}
\hline $\mathrm{H}(13 \mathrm{~A})$ & 2830 & 7955 & 2069 & 33 \\
\hline $\mathrm{H}(16 \mathrm{~A})$ & 2944 & 6344 & 981 & 30 \\
\hline $\mathrm{H}(18 \mathrm{~A})$ & 3204 & 7795 & -528 & 36 \\
\hline $\mathrm{H}(20 \mathrm{~A})$ & 3429 & 7951 & 1899 & 34 \\
\hline $\mathrm{H}(23 \mathrm{~A})$ & 3704 & 6917 & 5103 & 35 \\
\hline $\mathrm{H}(24 \mathrm{~A})$ & 3990 & 7055 & 6335 & 38 \\
\hline $\mathrm{H}(26 \mathrm{~A})$ & 3415 & 6061 & 7539 & 30 \\
\hline $\mathrm{H}(29 \mathrm{~A})$ & 2930 & 5331 & 7149 & 28 \\
\hline $\mathrm{H}(31 \mathrm{~A})$ & 2341 & 5125 & 5163 & 31 \\
\hline $\mathrm{H}(32 \mathrm{~A})$ & 2626 & 5706 & 4355 & 30 \\
\hline $\mathrm{H}(35 \mathrm{~A})$ & 4293 & 6502 & 7357 & 51 \\
\hline $\mathrm{H}(36 \mathrm{~A})$ & 4588 & 6491 & 8572 & 61 \\
\hline $\mathrm{H}(38 \mathrm{~A})$ & 3926 & 6796 & 9802 & 54 \\
\hline $\mathrm{H}(39 \mathrm{~A})$ & 3629 & 6805 & 8583 & 44 \\
\hline $\mathrm{H}(41 \mathrm{~A})$ & 2722 & 4119 & 7303 & 32 \\
\hline $\mathrm{H}(42 \mathrm{~A})$ & 2429 & 3462 & 8006 & 34 \\
\hline $\mathrm{H}(44 \mathrm{~A})$ & 1771 & 4659 & 7084 & 40 \\
\hline $\mathrm{H}(45 \mathrm{~A})$ & 2059 & 5281 & 6334 & 40 \\
\hline $\mathrm{H}(47 \mathrm{~A})$ & 3246 & 5944 & -710 & 55 \\
\hline $\mathrm{H}(47 \mathrm{~B})$ & 2956 & 5607 & -1112 & 55 \\
\hline $\mathrm{H}(47 \mathrm{C})$ & 3032 & 5621 & -142 & 55 \\
\hline $\mathrm{H}(48 \mathrm{~A})$ & 2576 & 6235 & -3 & 55 \\
\hline \multirow[t]{2}{*}{$\mathrm{H}(48 \mathrm{~B})$} & 2495 & 6221 & -972 & 55 \\
\hline & $\mathrm{x}$ & $\mathrm{y}$ & $\mathrm{z}$ & $\mathrm{U}(\mathrm{eq})$ \\
\hline $\mathrm{H}(48 \mathrm{C})$ & 2498 & 6945 & -489 & 55 \\
\hline $\mathrm{H}(49 \mathrm{~A})$ & 2831 & 7417 & -1429 & 56 \\
\hline $\mathrm{H}(49 \mathrm{~B})$ & 2813 & 6674 & -1874 & 56 \\
\hline $\mathrm{H}(49 \mathrm{C})$ & 3113 & 6996 & -1531 & 56 \\
\hline $\mathrm{H}(51 \mathrm{~A})$ & 3171 & 8990 & -384 & 77 \\
\hline $\mathrm{H}(51 \mathrm{~B})$ & 3359 & 9627 & 2 & 77 \\
\hline $\mathrm{H}(51 \mathrm{C})$ & 3128 & 9284 & 497 & 77 \\
\hline $\mathrm{H}(52 \mathrm{~A})$ & 3850 & 8212 & 194 & 82 \\
\hline $\mathrm{H}(52 \mathrm{~B})$ & 3806 & 8991 & -147 & 82 \\
\hline $\mathrm{H}(52 \mathrm{C})$ & 3621 & 8367 & -578 & 82 \\
\hline
\end{tabular}




\begin{tabular}{|c|c|c|c|c|}
\hline $\mathrm{H}(53 \mathrm{~A})$ & 3483 & 9127 & 1660 & 85 \\
\hline $\mathrm{H}(53 \mathrm{~B})$ & 3729 & 9425 & 1189 & 85 \\
\hline $\mathrm{H}(53 \mathrm{C})$ & 3759 & 8668 & 1601 & 85 \\
\hline $\mathrm{H}(55 \mathrm{~A})$ & 4805 & 7334 & 9669 & 110 \\
\hline $\mathrm{H}(55 \mathrm{~B})$ & 4793 & 7364 & 10631 & 110 \\
\hline $\mathrm{H}(55 \mathrm{C})$ & 4548 & 7715 & 10016 & 110 \\
\hline $\mathrm{H}(56 \mathrm{~A})$ & 4833 & 5958 & 10547 & 102 \\
\hline $\mathrm{H}(56 \mathrm{~B})$ & 4852 & 6125 & 9608 & 102 \\
\hline $\mathrm{H}(56 \mathrm{C})$ & 4618 & 5597 & 9852 & 102 \\
\hline $\mathrm{H}(57 \mathrm{~A})$ & 4226 & 6933 & 10816 & 110 \\
\hline $\mathrm{H}(57 \mathrm{~B})$ & 4509 & 6624 & 11302 & 110 \\
\hline $\mathrm{H}(57 \mathrm{C})$ & 4282 & 6110 & 10827 & 110 \\
\hline $\mathrm{H}(55 \mathrm{D})$ & 4340 & 7549 & 10598 & 116 \\
\hline $\mathrm{H}(55 \mathrm{E})$ & 4618 & 7598 & 10156 & 116 \\
\hline $\mathrm{H}(55 \mathrm{~F})$ & 4636 & 7299 & 11065 & 116 \\
\hline $\mathrm{H}(56 \mathrm{D})$ & 4840 & 6454 & 9696 & 104 \\
\hline $\mathrm{H}(56 \mathrm{E})$ & 4720 & 5737 & 10010 & 104 \\
\hline $\mathrm{H}(56 \mathrm{~F})$ & 4873 & 6284 & 10650 & 104 \\
\hline $\mathrm{H}(57 \mathrm{D})$ & 4449 & 6051 & 11287 & 105 \\
\hline $\mathrm{H}(57 \mathrm{E})$ & 4230 & 5753 & 10556 & 105 \\
\hline $\mathrm{H}(57 \mathrm{~F})$ & 4164 & 6468 & 10994 & 105 \\
\hline $\mathrm{H}(59 \mathrm{~A})$ & 2078 & 2642 & 8141 & 33 \\
\hline
\end{tabular}

\begin{tabular}{llllll}
\hline$x$ & $y$ & $z$ & $U(e q)$ \\
\hline
\end{tabular}

$\begin{array}{lrllr}\mathrm{H}(59 B) & 1867 & 2696 & 8814 & 33 \\ \mathrm{H}(59 \mathrm{C}) & 2160 & 3107 & 8940 & 33 \\ \mathrm{H}(60 \mathrm{~A}) & 1915 & 4341 & 9005 & 41 \\ \mathrm{H}(60 \mathrm{~B}) & 1667 & 3807 & 9120 & 41 \\ \mathrm{H}(60 \mathrm{C}) & 1619 & 4404 & 8440 & 41 \\ \mathrm{H}(61 \mathrm{~A}) & 1510 & 3714 & 7234 & 30 \\ \mathrm{H}(61 \mathrm{~B}) & 1482 & 3058 & 7815 & 30 \\ \mathrm{H}(61 \mathrm{C}) & 1676 & 3008 & 7100 & 30 \\ \mathrm{H}(59 \mathrm{D}) & 2050 & 2606 & 7946 & 101 \\ \mathrm{H}(59 \mathrm{E}) & 1848 & 2589 & 8646 & 101 \\ \mathrm{H}(59 \mathrm{~F}) & 2156 & 2925 & 8827 & 101\end{array}$




\begin{tabular}{|c|c|c|c|c|}
\hline $\mathrm{H}(60 \mathrm{D})$ & 2009 & 3992 & 9275 & 107 \\
\hline $\mathrm{H}(60 \mathrm{E})$ & 1678 & 3839 & 9145 & 107 \\
\hline $\mathrm{H}(60 \mathrm{~F})$ & 1800 & 4502 & 8720 & 107 \\
\hline H(61D) & 1489 & 3895 & 7513 & 108 \\
\hline $\mathrm{H}(61 \mathrm{E})$ & 1467 & 3156 & 7950 & 108 \\
\hline \multirow[t]{2}{*}{$\mathrm{H}(61 \mathrm{~F})$} & 1606 & 3208 & 7123 & 108 \\
\hline & $\mathrm{x}$ & $\mathrm{y}$ & $\mathrm{z}$ & $\mathrm{U}(\mathrm{eq})$ \\
\hline $\mathrm{H}(3 \mathrm{~B})$ & 4491 & 5048 & 8196 & 43 \\
\hline $\mathrm{H}(4 \mathrm{~B})$ & 4217 & 4142 & 8575 & 44 \\
\hline $\mathrm{H}(6 \mathrm{~B})$ & 4762 & 2716 & 7846 & 37 \\
\hline $\mathrm{H}(9 \mathrm{~B})$ & 3902 & 3268 & 8214 & 41 \\
\hline $\mathrm{H}(10 \mathrm{~B})$ & 3619 & 2379 & 8593 & 40 \\
\hline $\mathrm{H}(12 \mathrm{~B})$ & 4302 & 1169 & 9185 & 47 \\
\hline $\mathrm{H}(13 \mathrm{~B})$ & 4586 & 2045 & 8784 & 48 \\
\hline $\mathrm{H}(17 \mathrm{~B})$ & 4653 & 7569 & 6150 & 93 \\
\hline $\mathrm{H}(18 \mathrm{~B})$ & 4384 & 6928 & 5181 & 95 \\
\hline $\mathrm{H}(19 \mathrm{~B})$ & 4375 & 5716 & 5229 & 82 \\
\hline \multirow[t]{2}{*}{$\mathrm{H}(20 \mathrm{~B})$} & 4652 & 5135 & 6257 & 67 \\
\hline & $\mathrm{x}$ & $\mathrm{y}$ & $\mathrm{z}$ & $\mathrm{U}(\mathrm{eq})$ \\
\hline $\mathrm{H}(23 \mathrm{~B})$ & 5407 & 7968 & 7342 & 140 \\
\hline $\mathrm{H}(25 \mathrm{~B})$ & 5000 & 9795 & 7500 & 193 \\
\hline $\mathrm{H}(27 \mathrm{~A})$ & 5834 & 8624 & 7829 & 240 \\
\hline $\mathrm{H}(27 \mathrm{~B})$ & 5737 & 9281 & 8320 & 240 \\
\hline $\mathrm{H}(27 \mathrm{C})$ & 5954 & 9384 & 7669 & 240 \\
\hline $\mathrm{H}(28 \mathrm{~A})$ & 5621 & 10272 & 6807 & 243 \\
\hline $\mathrm{H}(28 \mathrm{~B})$ & 5462 & 10222 & 7601 & 243 \\
\hline $\mathrm{H}(28 \mathrm{C})$ & 5292 & 10092 & 6717 & 243 \\
\hline $\mathrm{H}(29 B)$ & 5828 & 9131 & 6280 & 250 \\
\hline $\mathrm{H}(29 \mathrm{C})$ & 5508 & 9178 & 5861 & 250 \\
\hline H(29D) & 5624 & 8472 & 6299 & 250 \\
\hline $\mathrm{H}(27 \mathrm{D})$ & 5769 & 10155 & 8010 & 244 \\
\hline
\end{tabular}




$\begin{array}{lrrrr}\mathrm{H}(27 \mathrm{E}) & 5444 & 10264 & 8118 & 244 \\ \mathrm{H}(27 \mathrm{~F}) & 5557 & 10483 & 7284 & 244 \\ \mathrm{H}(28 \mathrm{D}) & 5351 & 9024 & 6244 & 250 \\ \mathrm{H}(28 \mathrm{E}) & 5613 & 9535 & 6209 & 250 \\ \mathrm{H}(28 \mathrm{~F}) & 5310 & 9850 & 6309 & 250 \\ \mathrm{H}(29 \mathrm{E}) & 5940 & 9248 & 7698 & 238 \\ \mathrm{H}(29 \mathrm{~F}) & 5788 & 8624 & 7169 & 238 \\ \mathrm{H}(29 \mathrm{G}) & 5752 & 8695 & 8115 & 238 \\ \mathrm{H}(31 \mathrm{~B}) & 3364 & 1355 & 8565 & 77 \\ \mathrm{H}(31 \mathrm{C}) & 3412 & 1717 & 9444 & 77 \\ \mathrm{H}(31 \mathrm{D}) & 3330 & 908 & 9365 & 77 \\ \mathrm{H}(32 \mathrm{~B}) & 3737 & 555 & 10304 & 81 \\ \mathrm{H}(32 \mathrm{C}) & 3831 & 1351 & 10445 & 81 \\ \mathrm{H}(32 \mathrm{D}) & 4049 & 791 & 10171 & 81 \\ \mathrm{H}(33 \mathrm{~A}) & 3710 & 530 & 8146 & 84 \\ \mathrm{H}(33 \mathrm{~B}) & 3675 & 54 & 8923 & 84 \\ \mathrm{H}(33 \mathrm{C}) & 3981 & 312 & 8761 & 84\end{array}$

Table S8. Torsion angles $\left[{ }^{\circ}\right]$ for FR-1.

$\begin{array}{lc}\mathrm{C}(14 \mathrm{~A})-\mathrm{C}(1 \mathrm{~A})-\mathrm{C}(2 \mathrm{~A})-\mathrm{C}(3 \mathrm{~A}) & -179.2(3) \\ \mathrm{C}(15 \mathrm{~A})-\mathrm{C}(1 \mathrm{~A})-\mathrm{C}(2 \mathrm{~A})-\mathrm{C}(3 \mathrm{~A}) & -1.3(5) \\ \mathrm{C}(14 \mathrm{~A})-\mathrm{C}(1 \mathrm{~A})-\mathrm{C}(2 \mathrm{~A})-\mathrm{C}(7 \mathrm{~A}) & -1.1(5) \\ \mathrm{C}(15 \mathrm{~A})-\mathrm{C}(1 \mathrm{~A})-\mathrm{C}(2 \mathrm{~A})-\mathrm{C}(7 \mathrm{~A}) & 176.8(3) \\ \mathrm{C}(1 \mathrm{~A})-\mathrm{C}(2 \mathrm{~A})-\mathrm{C}(3 \mathrm{~A})-\mathrm{C}(4 \mathrm{~A}) & 177.0(3) \\ \mathrm{C}(7 \mathrm{~A})-\mathrm{C}(2 \mathrm{~A})-\mathrm{C}(3 \mathrm{~A})-\mathrm{C}(4 \mathrm{~A}) & -1.2(5) \\ \mathrm{C}(2 \mathrm{~A})-\mathrm{C}(3 \mathrm{~A})-\mathrm{C}(4 \mathrm{~A})-\mathrm{C}(5 \mathrm{~A}) & 1.2(5) \\ \mathrm{C}(3 \mathrm{~A})-\mathrm{C}(4 \mathrm{~A})-\mathrm{C}(5 \mathrm{~A})-\mathrm{C}(6 \mathrm{~A}) & -0.3(5) \\ \mathrm{C}(4 \mathrm{~A})-\mathrm{C}(5 \mathrm{~A})-\mathrm{C}(6 \mathrm{~A})-\mathrm{C}(7 \mathrm{~A}) & -0.5(5) \\ \mathrm{C}(5 \mathrm{~A})-\mathrm{C}(6 \mathrm{~A})-\mathrm{C}(7 \mathrm{~A})-\mathrm{C}(8 \mathrm{~A}) & -178.7(3) \\ \mathrm{C}(5 \mathrm{~A})-\mathrm{C}(6 \mathrm{~A})-\mathrm{C}(7 \mathrm{~A})-\mathrm{C}(2 \mathrm{~A}) & 0.4(5) \\ \mathrm{C}(1 \mathrm{~A})-\mathrm{C}(2 \mathrm{~A})-\mathrm{C}(7 \mathrm{~A})-\mathrm{C}(8 \mathrm{~A}) & 1.4(5) \\ \mathrm{C}(3 \mathrm{~A})-\mathrm{C}(2 \mathrm{~A})-\mathrm{C}(7 \mathrm{~A})-\mathrm{C}(8 \mathrm{~A}) & 179.5(3) \\ \mathrm{C}(1 \mathrm{~A})-\mathrm{C}(2 \mathrm{~A})-\mathrm{C}(7 \mathrm{~A})-\mathrm{C}(6 \mathrm{~A}) & -177.8(3) \\ \mathrm{C}(3 \mathrm{~A})-\mathrm{C}(2 \mathrm{~A})-\mathrm{C}(7 \mathrm{~A})-\mathrm{C}(6 \mathrm{~A}) & 0.4(5) \\ \mathrm{C}(6 \mathrm{~A})-\mathrm{C}(7 \mathrm{~A})-\mathrm{C}(8 \mathrm{~A})-\mathrm{C}(9 \mathrm{~A}) & 178.8(3)\end{array}$




\begin{tabular}{|c|c|}
\hline$C(2 A)-C(7 A)-C(8 A)-C(9 A)$ & $-0.4(5)$ \\
\hline $\mathrm{C}(6 \mathrm{~A})-\mathrm{C}(7 \mathrm{~A})-\mathrm{C}(8 \mathrm{~A})-\mathrm{C}(21 \mathrm{~A})$ & $-0.2(5)$ \\
\hline $\mathrm{C}(2 \mathrm{~A})-\mathrm{C}(7 \mathrm{~A})-\mathrm{C}(8 \mathrm{~A})-\mathrm{C}(21 \mathrm{~A})$ & $-179.3(3)$ \\
\hline$C(7 A)-C(8 A)-C(9 A)-C(10 A)$ & $-177.7(3)$ \\
\hline $\mathrm{C}(21 \mathrm{~A})-\mathrm{C}(8 \mathrm{~A})-\mathrm{C}(9 \mathrm{~A})-\mathrm{C}(10 \mathrm{~A})$ & $1.2(5)$ \\
\hline $\mathrm{C}(7 \mathrm{~A})-\mathrm{C}(8 \mathrm{~A})-\mathrm{C}(9 \mathrm{~A})-\mathrm{C}(14 \mathrm{~A})$ & $-0.9(5)$ \\
\hline $\mathrm{C}(21 \mathrm{~A})-\mathrm{C}(8 \mathrm{~A})-\mathrm{C}(9 \mathrm{~A})-\mathrm{C}(14 \mathrm{~A})$ & $178.0(3)$ \\
\hline $\mathrm{C}(8 \mathrm{~A})-\mathrm{C}(9 \mathrm{~A})-\mathrm{C}(10 \mathrm{~A})-\mathrm{C}(11 \mathrm{~A})$ & $177.7(3)$ \\
\hline$C(14 A)-C(9 A)-C(10 A)-C(11 A)$ & $0.9(5)$ \\
\hline$C(9 A)-C(10 A)-C(11 A)-C(12 A)$ & $-0.2(5)$ \\
\hline$C(10 A)-C(11 A)-C(12 A)-C(13 A)$ & $0.6(5)$ \\
\hline $\mathrm{C}(11 \mathrm{~A})-\mathrm{C}(12 \mathrm{~A})-\mathrm{C}(13 \mathrm{~A})-\mathrm{C}(14 \mathrm{~A})$ & $-1.8(5)$ \\
\hline $\mathrm{C}(2 \mathrm{~A})-\mathrm{C}(1 \mathrm{~A})-\mathrm{C}(14 \mathrm{~A})-\mathrm{C}(9 \mathrm{~A})$ & $-0.2(5)$ \\
\hline $\mathrm{C}(15 \mathrm{~A})-\mathrm{C}(1 \mathrm{~A})-\mathrm{C}(14 \mathrm{~A})-\mathrm{C}(9 \mathrm{~A})$ & $-178.0(3)$ \\
\hline$C(2 A)-C(1 A)-C(14 A)-C(13 A)$ & $179.8(3)$ \\
\hline $\mathrm{C}(15 \mathrm{~A})-\mathrm{C}(1 \mathrm{~A})-\mathrm{C}(14 \mathrm{~A})-\mathrm{C}(13 \mathrm{~A})$ & $2.0(5)$ \\
\hline $\mathrm{C}(8 \mathrm{~A})-\mathrm{C}(9 \mathrm{~A})-\mathrm{C}(14 \mathrm{~A})-\mathrm{C}(1 \mathrm{~A})$ & $1.2(5)$ \\
\hline$C(10 A)-C(9 A)-C(14 A)-C(1 A)$ & $178.1(3)$ \\
\hline $\mathrm{C}(8 \mathrm{~A})-\mathrm{C}(9 \mathrm{~A})-\mathrm{C}(14 \mathrm{~A})-\mathrm{C}(13 \mathrm{~A})$ & $-178.8(3)$ \\
\hline $\mathrm{C}(10 \mathrm{~A})-\mathrm{C}(9 \mathrm{~A})-\mathrm{C}(14 \mathrm{~A})-\mathrm{C}(13 \mathrm{~A})$ & $-1.9(4)$ \\
\hline $\mathrm{C}(12 \mathrm{~A})-\mathrm{C}(13 \mathrm{~A})-\mathrm{C}(14 \mathrm{~A})-\mathrm{C}(1 \mathrm{~A})$ & $-177.6(3)$ \\
\hline$C(12 A)-C(13 A)-C(14 A)-C(9 A)$ & $2.4(5)$ \\
\hline$C(14 A)-C(1 A)-C(15 A)-C(16 A)$ & $-105.2(4)$ \\
\hline$C(2 A)-C(1 A)-C(15 A)-C(16 A)$ & $76.9(4)$ \\
\hline$C(14 A)-C(1 A)-C(15 A)-C(20 A)$ & $72.9(4)$ \\
\hline$C(2 A)-C(1 A)-C(15 A)-C(20 A)$ & $-104.9(4)$ \\
\hline$C(20 A)-C(15 A)-C(16 A)-C(17 A)$ & $0.2(5)$ \\
\hline$C(1 A)-C(15 A)-C(16 A)-C(17 A)$ & 178.3(3) \\
\hline $\mathrm{C}(15 \mathrm{~A})-\mathrm{C}(16 \mathrm{~A})-\mathrm{C}(17 \mathrm{~A})-\mathrm{C}(18 \mathrm{~A})$ & $-0.8(5)$ \\
\hline $\mathrm{C}(15 \mathrm{~A})-\mathrm{C}(16 \mathrm{~A})-\mathrm{C}(17 \mathrm{~A})-\mathrm{C}(46 \mathrm{~A})$ & $178.4(3)$ \\
\hline $\mathrm{C}(16 \mathrm{~A})-\mathrm{C}(17 \mathrm{~A})-\mathrm{C}(18 \mathrm{~A})-\mathrm{C}(19 \mathrm{~A})$ & $0.4(5)$ \\
\hline $\mathrm{C}(46 \mathrm{~A})-\mathrm{C}(17 \mathrm{~A})-\mathrm{C}(18 \mathrm{~A})-\mathrm{C}(19 \mathrm{~A})$ & $-178.8(3)$ \\
\hline $\mathrm{C}(17 \mathrm{~A})-\mathrm{C}(18 \mathrm{~A})-\mathrm{C}(19 \mathrm{~A})-\mathrm{C}(20 \mathrm{~A})$ & $0.7(6)$ \\
\hline $\mathrm{C}(17 \mathrm{~A})-\mathrm{C}(18 \mathrm{~A})-\mathrm{C}(19 \mathrm{~A})-\mathrm{C}(50 \mathrm{~A})$ & $-177.2(3)$ \\
\hline$C(18 A)-C(19 A)-C(20 A)-C(15 A)$ & $-1.3(5)$ \\
\hline$C(50 A)-C(19 A)-C(20 A)-C(15 A)$ & $176.5(3)$ \\
\hline$C(16 A)-C(15 A)-C(20 A)-C(19 A)$ & $0.9(5)$ \\
\hline
\end{tabular}




\begin{tabular}{|c|c|}
\hline$C(1 A)-C(15 A)-C(20 A)-C(19 A)$ & $-177.3(3)$ \\
\hline $\mathrm{C}(7 \mathrm{~A})-\mathrm{C}(8 \mathrm{~A})-\mathrm{C}(21 \mathrm{~A})-\mathrm{C}(22 \mathrm{~A})$ & $75.2(4)$ \\
\hline $\mathrm{C}(9 \mathrm{~A})-\mathrm{C}(8 \mathrm{~A})-\mathrm{C}(21 \mathrm{~A})-\mathrm{C}(22 \mathrm{~A})$ & $-103.7(4)$ \\
\hline $\mathrm{C}(7 \mathrm{~A})-\mathrm{C}(8 \mathrm{~A})-\mathrm{C}(21 \mathrm{~A})-\mathrm{C}(33 \mathrm{~A})$ & $-107.2(4)$ \\
\hline $\mathrm{C}(9 \mathrm{~A})-\mathrm{C}(8 \mathrm{~A})-\mathrm{C}(21 \mathrm{~A})-\mathrm{C}(33 \mathrm{~A})$ & $73.8(4)$ \\
\hline $\mathrm{C}(33 \mathrm{~A})-\mathrm{C}(21 \mathrm{~A})-\mathrm{C}(22 \mathrm{~A})-\mathrm{C}(23 \mathrm{~A})$ & $-178.4(4)$ \\
\hline $\mathrm{C}(8 \mathrm{~A})-\mathrm{C}(21 \mathrm{~A})-\mathrm{C}(22 \mathrm{~A})-\mathrm{C}(23 \mathrm{~A})$ & $-0.4(6)$ \\
\hline $\mathrm{C}(33 \mathrm{~A})-\mathrm{C}(21 \mathrm{~A})-\mathrm{C}(22 \mathrm{~A})-\mathrm{C}(27 \mathrm{~A})$ & $1.3(4)$ \\
\hline$C(8 \mathrm{~A})-\mathrm{C}(21 \mathrm{~A})-\mathrm{C}(22 \mathrm{~A})-\mathrm{C}(27 \mathrm{~A})$ & $179.3(3)$ \\
\hline$C(27 A)-C(22 A)-C(23 A)-C(24 A)$ & $-1.1(5)$ \\
\hline$C(21 A)-C(22 A)-C(23 A)-C(24 A)$ & $178.6(3)$ \\
\hline$C(22 A)-C(23 A)-C(24 A)-C(25 A)$ & $0.1(5)$ \\
\hline$C(23 A)-C(24 A)-C(25 A)-C(26 A)$ & $-0.7(5)$ \\
\hline$C(23 A)-C(24 A)-C(25 A)-C(34 A)$ & $177.5(3)$ \\
\hline$C(24 A)-C(25 A)-C(26 A)-C(27 A)$ & $2.3(5)$ \\
\hline$C(34 A)-C(25 A)-C(26 A)-C(27 A)$ & $-175.9(3)$ \\
\hline$C(25 A)-C(26 A)-C(27 A)-C(22 A)$ & $-3.3(5)$ \\
\hline$C(25 A)-C(26 A)-C(27 A)-C(28 A)$ & $179.6(3)$ \\
\hline$C(23 A)-C(22 A)-C(27 A)-C(26 A)$ & $2.8(5)$ \\
\hline$C(21 A)-C(22 A)-C(27 A)-C(26 A)$ & $-177.0(3)$ \\
\hline $\mathrm{C}(23 \mathrm{~A})-\mathrm{C}(22 \mathrm{~A})-\mathrm{C}(27 \mathrm{~A})-\mathrm{C}(28 \mathrm{~A})$ & $-179.5(3)$ \\
\hline $\mathrm{C}(21 \mathrm{~A})-\mathrm{C}(22 \mathrm{~A})-\mathrm{C}(27 \mathrm{~A})-\mathrm{C}(28 \mathrm{~A})$ & $0.7(4)$ \\
\hline $\mathrm{C}(26 \mathrm{~A})-\mathrm{C}(27 \mathrm{~A})-\mathrm{C}(28 \mathrm{~A})-\mathrm{C}(29 \mathrm{~A})$ & $-6.8(6)$ \\
\hline $\mathrm{C}(22 \mathrm{~A})-\mathrm{C}(27 \mathrm{~A})-\mathrm{C}(28 \mathrm{~A})-\mathrm{C}(29 \mathrm{~A})$ & $175.9(3)$ \\
\hline$C(26 A)-C(27 A)-C(28 A)-C(33 A)$ & $174.9(3)$ \\
\hline$C(22 A)-C(27 A)-C(28 A)-C(33 A)$ & $-2.5(4)$ \\
\hline$C(33 A)-C(28 A)-C(29 A)-C(30 A)$ & $-2.9(5)$ \\
\hline$C(27 A)-C(28 A)-C(29 A)-C(30 A)$ & $179.0(3)$ \\
\hline$C(28 A)-C(29 A)-C(30 A)-C(31 A)$ & $0.7(5)$ \\
\hline$C(28 A)-C(29 A)-C(30 A)-C(40 A)$ & $-179.2(3)$ \\
\hline $\mathrm{C}(29 \mathrm{~A})-\mathrm{C}(30 \mathrm{~A})-\mathrm{C}(31 \mathrm{~A})-\mathrm{C}(32 \mathrm{~A})$ & $1.6(5)$ \\
\hline$C(40 A)-C(30 A)-C(31 A)-C(32 A)$ & $-178.5(3)$ \\
\hline$C(30 A)-C(31 A)-C(32 A)-C(33 A)$ & $-1.7(5)$ \\
\hline $\mathrm{C}(31 \mathrm{~A})-\mathrm{C}(32 \mathrm{~A})-\mathrm{C}(33 \mathrm{~A})-\mathrm{C}(28 \mathrm{~A})$ & $-0.5(5)$ \\
\hline$C(31 A)-C(32 A)-C(33 A)-C(21 A)$ & $177.0(3)$ \\
\hline$C(29 A)-C(28 A)-C(33 A)-C(32 A)$ & $2.8(5)$ \\
\hline $\mathrm{C}(27 \mathrm{~A})-\mathrm{C}(28 \mathrm{~A})-\mathrm{C}(33 \mathrm{~A})-\mathrm{C}(32 \mathrm{~A})$ & $-178.7(3)$ \\
\hline
\end{tabular}

177.3(3)

.2(4)

07.2(4)

$3.8(4)$

$8.4(4)$

.3(4)

79.3(3)

-1.1(5)

$0.1(5)$

(5)

.3(5)

75.9(3)

$-3.3(5)$

(3)

177.0(3)

179.5(3)

$0.7(4)$

$-6.8(6)$

75.9(3)

74.9(3)

$2.5(4)$

$2.9(5)$

79.0(3)

$0.7(5)$

179.2(3)

1.7(5)

$0.5(5)$

.0(3)

178.7(3) 


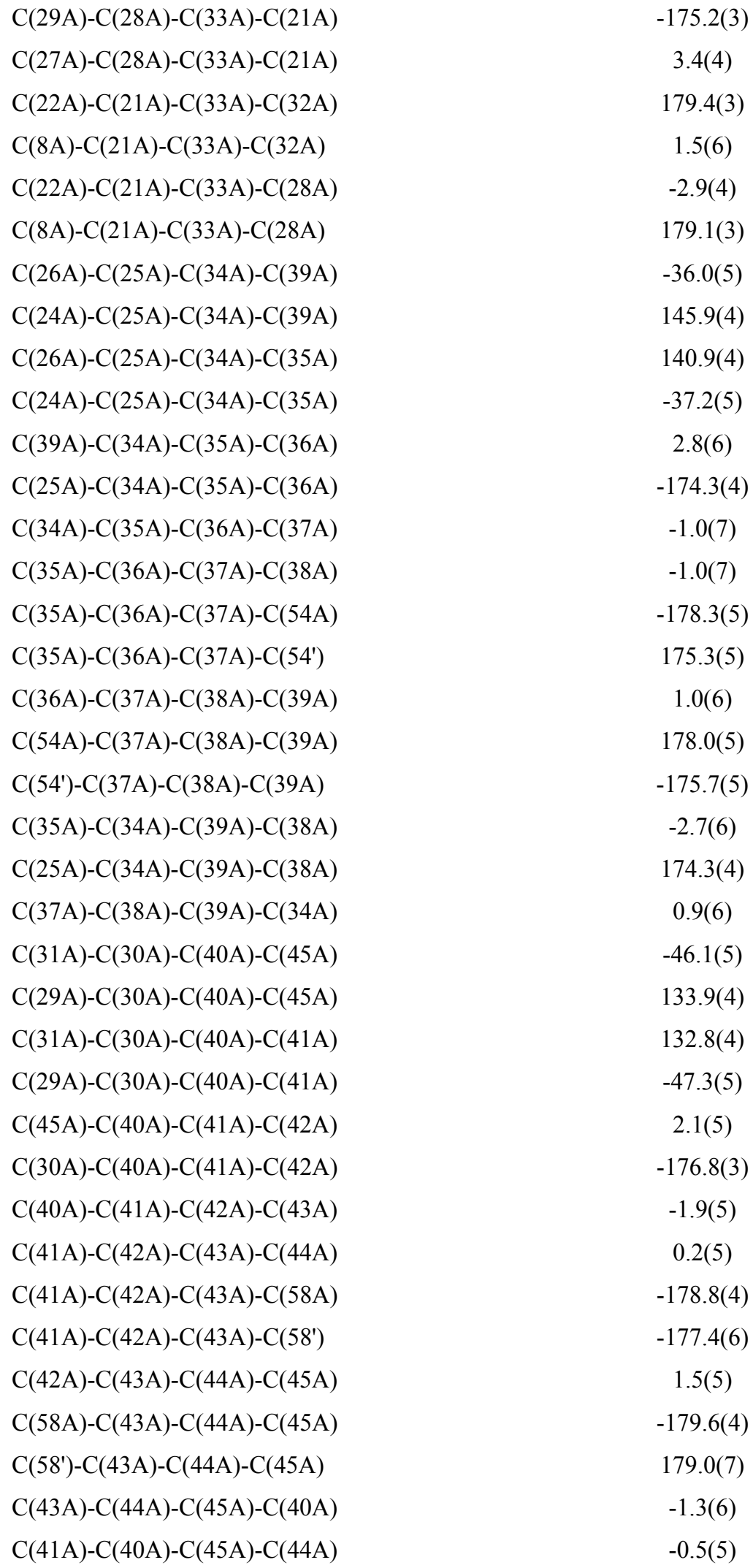




\begin{tabular}{|c|c|}
\hline$C(30 A)-C(40 A)-C(45 A)-C(44 A)$ & $178.5(3)$ \\
\hline $\mathrm{C}(18 \mathrm{~A})-\mathrm{C}(17 \mathrm{~A})-\mathrm{C}(46 \mathrm{~A})-\mathrm{C}(49 \mathrm{~A})$ & $-3.8(5)$ \\
\hline$C(16 A)-C(17 A)-C(46 A)-C(49 A)$ & 177.1(3) \\
\hline $\mathrm{C}(18 \mathrm{~A})-\mathrm{C}(17 \mathrm{~A})-\mathrm{C}(46 \mathrm{~A})-\mathrm{C}(48 \mathrm{~A})$ & $-124.8(4)$ \\
\hline$C(16 A)-C(17 A)-C(46 A)-C(48 A)$ & $56.1(4)$ \\
\hline $\mathrm{C}(18 \mathrm{~A})-\mathrm{C}(17 \mathrm{~A})-\mathrm{C}(46 \mathrm{~A})-\mathrm{C}(47 \mathrm{~A})$ & $115.1(4)$ \\
\hline$C(16 A)-C(17 A)-C(46 A)-C(47 A)$ & $-64.0(4)$ \\
\hline$C(20 A)-C(19 A)-C(50 A)-C(51 A)$ & $-117.2(4)$ \\
\hline $\mathrm{C}(18 \mathrm{~A})-\mathrm{C}(19 \mathrm{~A})-\mathrm{C}(50 \mathrm{~A})-\mathrm{C}(51 \mathrm{~A})$ & $60.5(4)$ \\
\hline$C(20 A)-C(19 A)-C(50 A)-C(53 A)$ & $1.9(5)$ \\
\hline$C(18 A)-C(19 A)-C(50 A)-C(53 A)$ & $179.6(4)$ \\
\hline$C(20 A)-C(19 A)-C(50 A)-C(52 A)$ & $123.0(4)$ \\
\hline $\mathrm{C}(18 \mathrm{~A})-\mathrm{C}(19 \mathrm{~A})-\mathrm{C}(50 \mathrm{~A})-\mathrm{C}(52 \mathrm{~A})$ & $-59.2(5)$ \\
\hline$C(36 \mathrm{~A})-\mathrm{C}(37 \mathrm{~A})-\mathrm{C}(54 \mathrm{~A})-\mathrm{C}(55 \mathrm{~A})$ & $73.9(7)$ \\
\hline $\mathrm{C}(38 \mathrm{~A})-\mathrm{C}(37 \mathrm{~A})-\mathrm{C}(54 \mathrm{~A})-\mathrm{C}(55 \mathrm{~A})$ & $-103.1(7)$ \\
\hline$C(36 \mathrm{~A})-\mathrm{C}(37 \mathrm{~A})-\mathrm{C}(54 \mathrm{~A})-\mathrm{C}(57 \mathrm{~A})$ & $-167.7(6)$ \\
\hline $\mathrm{C}(38 \mathrm{~A})-\mathrm{C}(37 \mathrm{~A})-\mathrm{C}(54 \mathrm{~A})-\mathrm{C}(57 \mathrm{~A})$ & $15.3(9)$ \\
\hline$C(36 A)-C(37 A)-C(54 A)-C(56 A)$ & $-48.0(7)$ \\
\hline $\mathrm{C}(38 \mathrm{~A})-\mathrm{C}(37 \mathrm{~A})-\mathrm{C}(54 \mathrm{~A})-\mathrm{C}(56 \mathrm{~A})$ & $135.0(7)$ \\
\hline $\mathrm{C}(36 \mathrm{~A})-\mathrm{C}(37 \mathrm{~A})-\mathrm{C}\left(54^{\prime}\right)-\mathrm{C}\left(55^{\prime}\right)$ & $102.7(7)$ \\
\hline $\mathrm{C}(38 \mathrm{~A})-\mathrm{C}(37 \mathrm{~A})-\mathrm{C}\left(54^{\prime}\right)-\mathrm{C}\left(55^{\prime}\right)$ & $-81.0(7)$ \\
\hline$C(36 A)-C(37 A)-C\left(54^{\prime}\right)-C\left(57^{\prime}\right)$ & $-136.9(6)$ \\
\hline$C(38 \mathrm{~A})-\mathrm{C}(37 \mathrm{~A})-\mathrm{C}\left(54^{\prime}\right)-\mathrm{C}\left(57^{\prime}\right)$ & $39.4(8)$ \\
\hline $\mathrm{C}(36 \mathrm{~A})-\mathrm{C}(37 \mathrm{~A})-\mathrm{C}\left(54^{\prime}\right)-\mathrm{C}\left(56^{\prime}\right)$ & $-15.9(8)$ \\
\hline $\mathrm{C}(38 \mathrm{~A})-\mathrm{C}(37 \mathrm{~A})-\mathrm{C}\left(54^{\prime}\right)-\mathrm{C}\left(56^{\prime}\right)$ & $160.4(6)$ \\
\hline $\mathrm{C}(42 \mathrm{~A})-\mathrm{C}(43 \mathrm{~A})-\mathrm{C}(58 \mathrm{~A})-\mathrm{C}(59 \mathrm{~A})$ & $-7.2(7)$ \\
\hline $\mathrm{C}(44 \mathrm{~A})-\mathrm{C}(43 \mathrm{~A})-\mathrm{C}(58 \mathrm{~A})-\mathrm{C}(59 \mathrm{~A})$ & $173.9(5)$ \\
\hline $\mathrm{C}(42 \mathrm{~A})-\mathrm{C}(43 \mathrm{~A})-\mathrm{C}(58 \mathrm{~A})-\mathrm{C}(60 \mathrm{~A})$ & $113.9(5)$ \\
\hline $\mathrm{C}(44 \mathrm{~A})-\mathrm{C}(43 \mathrm{~A})-\mathrm{C}(58 \mathrm{~A})-\mathrm{C}(60 \mathrm{~A})$ & $-65.0(6)$ \\
\hline $\mathrm{C}(42 \mathrm{~A})-\mathrm{C}(43 \mathrm{~A})-\mathrm{C}(58 \mathrm{~A})-\mathrm{C}(61 \mathrm{~A})$ & $-127.0(5)$ \\
\hline $\mathrm{C}(44 \mathrm{~A})-\mathrm{C}(43 \mathrm{~A})-\mathrm{C}(58 \mathrm{~A})-\mathrm{C}(61 \mathrm{~A})$ & $54.1(6)$ \\
\hline $\mathrm{C}(42 \mathrm{~A})-\mathrm{C}(43 \mathrm{~A})-\mathrm{C}\left(58^{\prime}\right)-\mathrm{C}\left(59^{\prime}\right)$ & $-21.3(10)$ \\
\hline $\mathrm{C}(44 \mathrm{~A})-\mathrm{C}(43 \mathrm{~A})-\mathrm{C}\left(58^{\prime}\right)-\mathrm{C}\left(59^{\prime}\right)$ & $161.3(7)$ \\
\hline $\mathrm{C}(42 \mathrm{~A})-\mathrm{C}(43 \mathrm{~A})-\mathrm{C}\left(58^{\prime}\right)-\mathrm{C}\left(61^{\prime}\right)$ & $-142.3(7)$ \\
\hline $\mathrm{C}(44 \mathrm{~A})-\mathrm{C}(43 \mathrm{~A})-\mathrm{C}\left(58^{\prime}\right)-\mathrm{C}\left(61^{\prime}\right)$ & $40.3(9)$ \\
\hline$C(42 A)-C(43 A)-C\left(58^{\prime}\right)-C\left(60^{\prime}\right)$ & $98.8(8)$ \\
\hline $\mathrm{C}(44 \mathrm{~A})-\mathrm{C}(43 \mathrm{~A})-\mathrm{C}\left(58^{\prime}\right)-\mathrm{C}\left(60^{\prime}\right)$ & $-78.6(8)$ \\
\hline
\end{tabular}

78.5(3)

$8(5)$

24.8(4)

56.1(4)

$5.1(4)$

$0.5(4)$

$.9(5)$

79.6(4)

$59.2(5)$

73.9(7)

03.1(7)

(6.7.

48.0(7)

$135.0(7)$

$02.7(7)$

$-81.0(7)$

36.9(6)

$39.4(8)$

$15.9(8)$

60.4(6)

$-7.2(7)$

$73.9(5)$

$13.9(5)$

$65.0(6)$

127.0(5)

54.1(6)

21.3(10)

61.3(7)

142.3(7)

40.3(9)

$-78.6(8)$ 


\begin{tabular}{|c|c|}
\hline $\mathrm{C}(2 \mathrm{~B}) \# 1-\mathrm{C}(1 \mathrm{~B})-\mathrm{C}(2 \mathrm{~B})-\mathrm{C}(3 \mathrm{~B})$ & $-178.9(5)$ \\
\hline $\mathrm{C}(14 \mathrm{~B})-\mathrm{C}(1 \mathrm{~B})-\mathrm{C}(2 \mathrm{~B})-\mathrm{C}(3 \mathrm{~B})$ & $1.1(5)$ \\
\hline $\mathrm{C}(2 \mathrm{~B}) \# 1-\mathrm{C}(1 \mathrm{~B})-\mathrm{C}(2 \mathrm{~B})-\mathrm{C}(7 \mathrm{~B})$ & $0.04(17)$ \\
\hline $\mathrm{C}(14 \mathrm{~B})-\mathrm{C}(1 \mathrm{~B})-\mathrm{C}(2 \mathrm{~B})-\mathrm{C}(7 \mathrm{~B})$ & $-179.96(17)$ \\
\hline $\mathrm{C}(7 \mathrm{~B})-\mathrm{C}(2 \mathrm{~B})-\mathrm{C}(3 \mathrm{~B})-\mathrm{C}(4 \mathrm{~B})$ & $0.5(5)$ \\
\hline $\mathrm{C}(1 \mathrm{~B})-\mathrm{C}(2 \mathrm{~B})-\mathrm{C}(3 \mathrm{~B})-\mathrm{C}(4 \mathrm{~B})$ & $179.4(3)$ \\
\hline $\mathrm{C}(2 \mathrm{~B})-\mathrm{C}(3 \mathrm{~B})-\mathrm{C}(4 \mathrm{~B})-\mathrm{C}(5 \mathrm{~B})$ & $-0.2(6)$ \\
\hline $\mathrm{C}(3 \mathrm{~B})-\mathrm{C}(4 \mathrm{~B})-\mathrm{C}(5 \mathrm{~B})-\mathrm{C}(6 \mathrm{~B})$ & $0.3(6)$ \\
\hline $\mathrm{C}(3 \mathrm{~B})-\mathrm{C}(4 \mathrm{~B})-\mathrm{C}(5 \mathrm{~B})-\mathrm{C}(8 \mathrm{~B})$ & $-178.7(4)$ \\
\hline $\mathrm{C}(4 \mathrm{~B})-\mathrm{C}(5 \mathrm{~B})-\mathrm{C}(6 \mathrm{~B})-\mathrm{C}(7 \mathrm{~B})$ & $-0.6(5)$ \\
\hline $\mathrm{C}(8 \mathrm{~B})-\mathrm{C}(5 \mathrm{~B})-\mathrm{C}(6 \mathrm{~B})-\mathrm{C}(7 \mathrm{~B})$ & $178.3(3)$ \\
\hline $\mathrm{C}(5 \mathrm{~B})-\mathrm{C}(6 \mathrm{~B})-\mathrm{C}(7 \mathrm{~B})-\mathrm{C}(2 \mathrm{~B})$ & $1.0(5)$ \\
\hline$C(5 B)-C(6 B)-C(7 B)-C(7 B) \# 1$ & $-178.9(4)$ \\
\hline$C(3 B)-C(2 B)-C(7 B)-C(6 B)$ & $-0.9(5)$ \\
\hline$C(1 B)-C(2 B)-C(7 B)-C(6 B)$ & $180.0(3)$ \\
\hline $\mathrm{C}(3 \mathrm{~B})-\mathrm{C}(2 \mathrm{~B})-\mathrm{C}(7 \mathrm{~B})-\mathrm{C}(7 \mathrm{~B}) \# 1$ & $179.0(4)$ \\
\hline $\mathrm{C}(1 \mathrm{~B})-\mathrm{C}(2 \mathrm{~B})-\mathrm{C}(7 \mathrm{~B})-\mathrm{C}(7 \mathrm{~B}) \# 1$ & $-0.1(4)$ \\
\hline $\mathrm{C}(6 \mathrm{~B})-\mathrm{C}(5 \mathrm{~B})-\mathrm{C}(8 \mathrm{~B})-\mathrm{C}(13 \mathrm{~B})$ & $32.0(5)$ \\
\hline $\mathrm{C}(4 \mathrm{~B})-\mathrm{C}(5 \mathrm{~B})-\mathrm{C}(8 \mathrm{~B})-\mathrm{C}(13 \mathrm{~B})$ & $-149.1(4)$ \\
\hline $\mathrm{C}(6 \mathrm{~B})-\mathrm{C}(5 \mathrm{~B})-\mathrm{C}(8 \mathrm{~B})-\mathrm{C}(9 \mathrm{~B})$ & $-147.5(4)$ \\
\hline $\mathrm{C}(4 \mathrm{~B})-\mathrm{C}(5 \mathrm{~B})-\mathrm{C}(8 \mathrm{~B})-\mathrm{C}(9 \mathrm{~B})$ & $31.5(5)$ \\
\hline $\mathrm{C}(13 \mathrm{~B})-\mathrm{C}(8 \mathrm{~B})-\mathrm{C}(9 \mathrm{~B})-\mathrm{C}(10 \mathrm{~B})$ & $1.6(6)$ \\
\hline $\mathrm{C}(5 \mathrm{~B})-\mathrm{C}(8 \mathrm{~B})-\mathrm{C}(9 \mathrm{~B})-\mathrm{C}(10 \mathrm{~B})$ & $-178.9(3)$ \\
\hline $\mathrm{C}(8 \mathrm{~B})-\mathrm{C}(9 \mathrm{~B})-\mathrm{C}(10 \mathrm{~B})-\mathrm{C}(11 \mathrm{~B})$ & $0.0(6)$ \\
\hline $\mathrm{C}(9 \mathrm{~B})-\mathrm{C}(10 \mathrm{~B})-\mathrm{C}(11 \mathrm{~B})-\mathrm{C}(12 \mathrm{~B})$ & $-1.0(6)$ \\
\hline $\mathrm{C}(9 \mathrm{~B})-\mathrm{C}(10 \mathrm{~B})-\mathrm{C}(11 \mathrm{~B})-\mathrm{C}(30)$ & $177.7(4)$ \\
\hline $\mathrm{C}(10 \mathrm{~B})-\mathrm{C}(11 \mathrm{~B})-\mathrm{C}(12 \mathrm{~B})-\mathrm{C}(13 \mathrm{~B})$ & $0.3(6)$ \\
\hline$C(30)-C(11 B)-C(12 B)-C(13 B)$ & $-178.4(4)$ \\
\hline$C(11 B)-C(12 B)-C(13 B)-C(8 B)$ & $1.4(6)$ \\
\hline $\mathrm{C}(9 \mathrm{~B})-\mathrm{C}(8 \mathrm{~B})-\mathrm{C}(13 \mathrm{~B})-\mathrm{C}(12 \mathrm{~B})$ & $-2.3(6)$ \\
\hline$C(5 B)-C(8 B)-C(13 B)-C(12 B)$ & $178.2(4)$ \\
\hline $\mathrm{C}(2 \mathrm{~B})-\mathrm{C}(1 \mathrm{~B})-\mathrm{C}(14 \mathrm{~B})-\mathrm{C}(15 \mathrm{~B}) \# 1$ & $99.5(3)$ \\
\hline $\mathrm{C}(2 \mathrm{~B}) \# 1-\mathrm{C}(1 \mathrm{~B})-\mathrm{C}(14 \mathrm{~B})-\mathrm{C}(15 \mathrm{~B}) \# 1$ & $-80.5(3)$ \\
\hline$C(2 B)-C(1 B)-C(14 B)-C(15 B)$ & $-80.5(3)$ \\
\hline $\mathrm{C}(2 \mathrm{~B}) \# 1-\mathrm{C}(1 \mathrm{~B})-\mathrm{C}(14 \mathrm{~B})-\mathrm{C}(15 \mathrm{~B})$ & $99.5(3)$ \\
\hline$C(15 B) \# 1-C(14 B)-C(15 B)-C(16 B)$ & $1.1(3)$ \\
\hline
\end{tabular}

8.9(5)

$.04(17)$

179.96(17)

$0.5(5)$

$-0.2(6)$

$0.3(6)$

78.7(4)

$.0(5)$

78.9(4)

9(5)

$79.0(4)$

$0.1(4)$

$2.0(5)$

$.5(5)$

.6(6)

(3)

$1.0(6)$

77.7(4)

$0.3(6)$

1.4(6)

.3(6)

78.2(4)

$9.5(3)$

$80.5(3)$

9.5(3)

1.1(3) 


\begin{tabular}{|c|c|}
\hline$C(1 B)-C(14 B)-C(15 B)-C(16 B)$ & $-178.9(3)$ \\
\hline $\mathrm{C}(15 \mathrm{~B}) \# 1-\mathrm{C}(14 \mathrm{~B})-\mathrm{C}(15 \mathrm{~B})-\mathrm{C}(20 \mathrm{~B})$ & $-179.6(4)$ \\
\hline $\mathrm{C}(1 \mathrm{~B})-\mathrm{C}(14 \mathrm{~B})-\mathrm{C}(15 \mathrm{~B})-\mathrm{C}(20 \mathrm{~B})$ & $0.4(4)$ \\
\hline $\mathrm{C}(14 \mathrm{~B})-\mathrm{C}(15 \mathrm{~B})-\mathrm{C}(16 \mathrm{~B})-\mathrm{C}(21 \mathrm{~B})$ & $-2.3(6)$ \\
\hline $\mathrm{C}(20 \mathrm{~B})-\mathrm{C}(15 \mathrm{~B})-\mathrm{C}(16 \mathrm{~B})-\mathrm{C}(21 \mathrm{~B})$ & $178.4(4)$ \\
\hline $\mathrm{C}(14 \mathrm{~B})-\mathrm{C}(15 \mathrm{~B})-\mathrm{C}(16 \mathrm{~B})-\mathrm{C}(17 \mathrm{~B})$ & $178.4(4)$ \\
\hline $\mathrm{C}(20 \mathrm{~B})-\mathrm{C}(15 \mathrm{~B})-\mathrm{C}(16 \mathrm{~B})-\mathrm{C}(17 \mathrm{~B})$ & $-0.9(6)$ \\
\hline $\mathrm{C}(21 \mathrm{~B})-\mathrm{C}(16 \mathrm{~B})-\mathrm{C}(17 \mathrm{~B})-\mathrm{C}(18 \mathrm{~B})$ & $-178.5(5)$ \\
\hline $\mathrm{C}(15 \mathrm{~B})-\mathrm{C}(16 \mathrm{~B})-\mathrm{C}(17 \mathrm{~B})-\mathrm{C}(18 \mathrm{~B})$ & $0.8(7)$ \\
\hline$C(16 B)-C(17 B)-C(18 B)-C(19 B)$ & $0.1(9)$ \\
\hline$C(17 B)-C(18 B)-C(19 B)-C(20 B)$ & $-1.0(9)$ \\
\hline$C(18 B)-C(19 B)-C(20 B)-C(15 B)$ & $0.8(8)$ \\
\hline$C(14 B)-C(15 B)-C(20 B)-C(19 B)$ & $-179.1(4)$ \\
\hline$C(16 B)-C(15 B)-C(20 B)-C(19 B)$ & $0.2(7)$ \\
\hline$C(15 B)-C(16 B)-C(21 B)-C(16 B) \# 1$ & $1.1(3)$ \\
\hline$C(17 B)-C(16 B)-C(21 B)-C(16 B) \# 1$ & $-179.6(5)$ \\
\hline$C(15 B)-C(16 B)-C(21 B)-C(22 B)$ & $-178.9(3)$ \\
\hline $\mathrm{C}(17 \mathrm{~B})-\mathrm{C}(16 \mathrm{~B})-\mathrm{C}(21 \mathrm{~B})-\mathrm{C}(22 \mathrm{~B})$ & $0.5(5)$ \\
\hline $\mathrm{C}(16 \mathrm{~B}) \# 1-\mathrm{C}(21 \mathrm{~B})-\mathrm{C}(22 \mathrm{~B})-\mathrm{C}(23 \mathrm{~B}) \# 1$ & $-115.5(5)$ \\
\hline $\mathrm{C}(16 \mathrm{~B})-\mathrm{C}(21 \mathrm{~B})-\mathrm{C}(22 \mathrm{~B})-\mathrm{C}(23 \mathrm{~B}) \# 1$ & $64.5(5)$ \\
\hline $\mathrm{C}(16 \mathrm{~B}) \# 1-\mathrm{C}(21 \mathrm{~B})-\mathrm{C}(22 \mathrm{~B})-\mathrm{C}(23 \mathrm{~B})$ & $64.5(5)$ \\
\hline$C(16 B)-C(21 B)-C(22 B)-C(23 B)$ & $-115.5(5)$ \\
\hline $\mathrm{C}(23 \mathrm{~B}) \# 1-\mathrm{C}(22 \mathrm{~B})-\mathrm{C}(23 \mathrm{~B})-\mathrm{C}(24 \mathrm{~B})$ & $0.6(9)$ \\
\hline $\mathrm{C}(21 \mathrm{~B})-\mathrm{C}(22 \mathrm{~B})-\mathrm{C}(23 \mathrm{~B})-\mathrm{C}(24 \mathrm{~B})$ & $-179.4(9)$ \\
\hline $\mathrm{C}(22 \mathrm{~B})-\mathrm{C}(23 \mathrm{~B})-\mathrm{C}(24 \mathrm{~B})-\mathrm{C}(25 \mathrm{~B})$ & $-1.1(16)$ \\
\hline $\mathrm{C}(22 \mathrm{~B})-\mathrm{C}(23 \mathrm{~B})-\mathrm{C}(24 \mathrm{~B})-\mathrm{C}\left(26^{\prime}\right)$ & $174.4(12)$ \\
\hline $\mathrm{C}(22 \mathrm{~B})-\mathrm{C}(23 \mathrm{~B})-\mathrm{C}(24 \mathrm{~B})-\mathrm{C}(26 \mathrm{~B})$ & $-169.3(9)$ \\
\hline $\mathrm{C}(23 \mathrm{~B})-\mathrm{C}(24 \mathrm{~B})-\mathrm{C}(25 \mathrm{~B})-\mathrm{C}(24 \mathrm{~B}) \# 1$ & $0.6(8)$ \\
\hline $\mathrm{C}\left(26^{\prime}\right)-\mathrm{C}(24 \mathrm{~B})-\mathrm{C}(25 \mathrm{~B})-\mathrm{C}(24 \mathrm{~B}) \# 1$ & $-175.6(13)$ \\
\hline $\mathrm{C}(26 \mathrm{~B})-\mathrm{C}(24 \mathrm{~B})-\mathrm{C}(25 \mathrm{~B})-\mathrm{C}(24 \mathrm{~B}) \# 1$ & $166.3(16)$ \\
\hline$C(23 B)-C(24 B)-C(26 B)-C(27 B)$ & $-72.9(13)$ \\
\hline$C(25 B)-C(24 B)-C(26 B)-C(27 B)$ & $121.0(13)$ \\
\hline$C(23 B)-C(24 B)-C(26 B)-C(29 B)$ & $52.1(14)$ \\
\hline$C(25 B)-C(24 B)-C(26 B)-C(29 B)$ & $-114.1(14)$ \\
\hline $\mathrm{C}(23 \mathrm{~B})-\mathrm{C}(24 \mathrm{~B})-\mathrm{C}(26 \mathrm{~B})-\mathrm{C}(28 \mathrm{~B})$ & $171.0(11)$ \\
\hline $\mathrm{C}(25 \mathrm{~B})-\mathrm{C}(24 \mathrm{~B})-\mathrm{C}(26 \mathrm{~B})-\mathrm{C}(28 \mathrm{~B})$ & $4.9(16)$ \\
\hline $\mathrm{C}(23 \mathrm{~B})-\mathrm{C}(24 \mathrm{~B})-\mathrm{C}\left(26^{\prime}\right)-\mathrm{C}\left(27^{\prime}\right)$ & $-150.7(13)$ \\
\hline
\end{tabular}




$\begin{array}{lc}\mathrm{C}(25 \mathrm{~B})-\mathrm{C}(24 \mathrm{~B})-\mathrm{C}\left(26^{\prime}\right)-\mathrm{C}\left(27^{\prime}\right) & 24.9(18) \\ \mathrm{C}(23 \mathrm{~B})-\mathrm{C}(24 \mathrm{~B})-\mathrm{C}\left(26^{\prime}\right)-\mathrm{C}\left(29^{\prime}\right) & -16.1(18) \\ \mathrm{C}(25 \mathrm{~B})-\mathrm{C}(24 \mathrm{~B})-\mathrm{C}\left(26^{\prime}\right)-\mathrm{C}\left(29^{\prime}\right) & 159.5(11) \\ \mathrm{C}(23 \mathrm{~B})-\mathrm{C}(24 \mathrm{~B})-\mathrm{C}\left(26^{\prime}\right)-\mathrm{C}\left(28^{\prime}\right) & 94.2(16) \\ \mathrm{C}(25 \mathrm{~B})-\mathrm{C}(24 \mathrm{~B})-\mathrm{C}\left(26^{\prime}\right)-\mathrm{C}\left(28^{\prime}\right) & -90.3(11) \\ \mathrm{C}(10 \mathrm{~B})-\mathrm{C}(11 \mathrm{~B})-\mathrm{C}(30)-\mathrm{C}(33) & 120.7(4) \\ \mathrm{C}(12 \mathrm{~B})-\mathrm{C}(11 \mathrm{~B})-\mathrm{C}(30)-\mathrm{C}(33) & -60.7(5) \\ \mathrm{C}(10 \mathrm{~B})-\mathrm{C}(11 \mathrm{~B})-\mathrm{C}(30)-\mathrm{C}(32) & -120.4(4) \\ \mathrm{C}(12 \mathrm{~B})-\mathrm{C}(11 \mathrm{~B})-\mathrm{C}(30)-\mathrm{C}(32) & 58.2(5) \\ \mathrm{C}(10 \mathrm{~B})-\mathrm{C}(11 \mathrm{~B})-\mathrm{C}(30)-\mathrm{C}(31) & -0.1(5) \\ \mathrm{C}(12 \mathrm{~B})-\mathrm{C}(11 \mathrm{~B})-\mathrm{C}(30)-\mathrm{C}(31) & 178.6(4)\end{array}$

8.2 X-ray crystallographic data for FR-2.

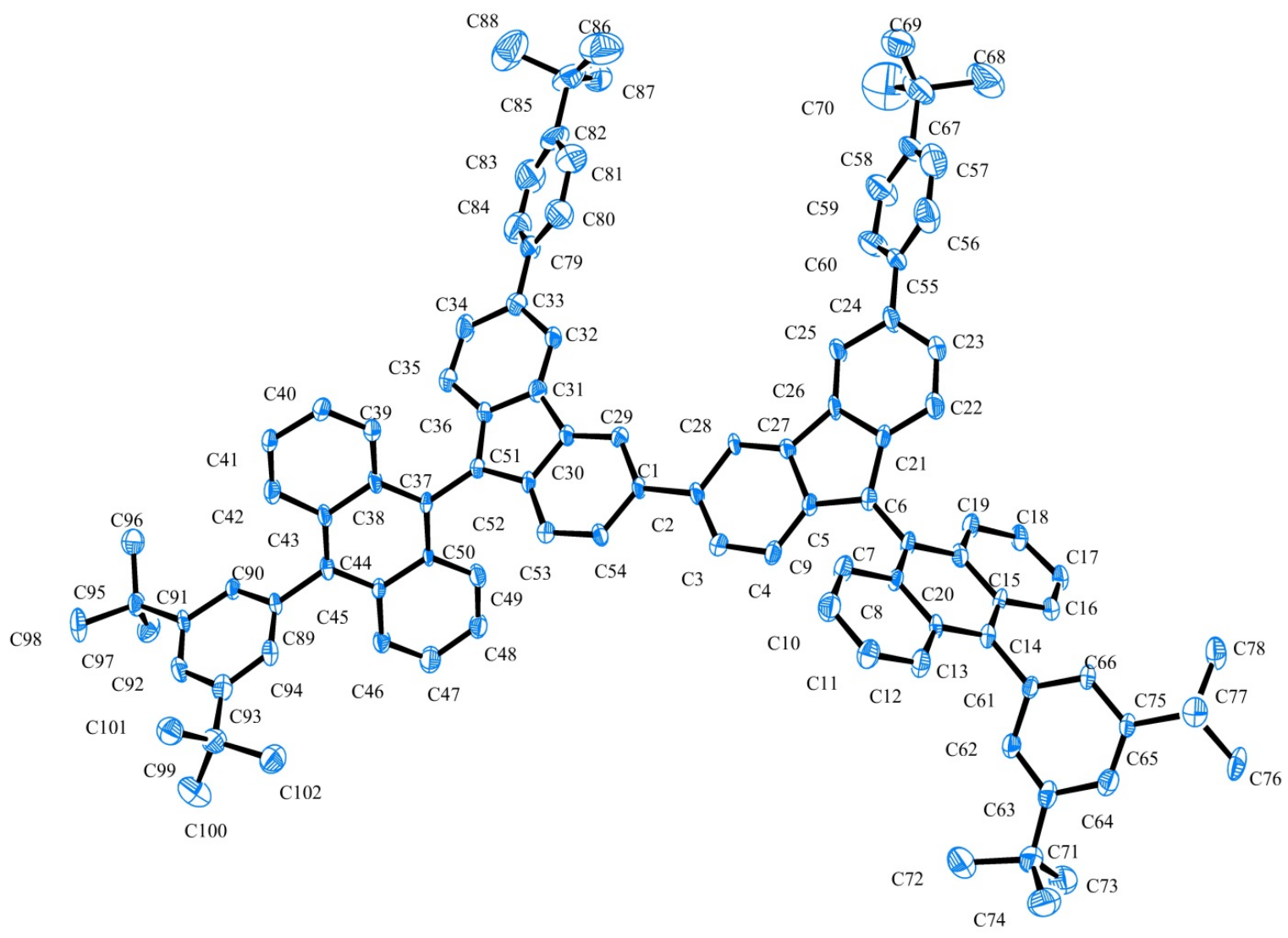

Molecule's view 
Table S9. Sample and crystal data for FR-2.

\begin{tabular}{|c|c|c|}
\hline Identification code & \multicolumn{2}{|l|}{ g218 } \\
\hline Chemical formula & \multicolumn{2}{|l|}{$\mathrm{C}_{102} \mathrm{H}_{96}$} \\
\hline Formula weight & \multicolumn{2}{|l|}{$1321.78 \mathrm{~g} / \mathrm{mol}$} \\
\hline Temperature & \multicolumn{2}{|l|}{$100(2) \mathrm{K}$} \\
\hline Wavelength & \multicolumn{2}{|l|}{$1.54178 \AA$} \\
\hline Crystal size & \multicolumn{2}{|c|}{$0.200 \times 0.244 \times 0.597 \mathrm{~mm}$} \\
\hline Crystal system & \multicolumn{2}{|l|}{ monoclinic } \\
\hline Space group & \multicolumn{2}{|l|}{$\mathrm{C} 12 / \mathrm{c} 1$} \\
\hline \multirow[t]{3}{*}{ Unit cell dimensions } & $\mathrm{a}=53.095(4) \AA$ & $\alpha=90^{\circ}$ \\
\hline & $\mathrm{b}=10.2240(8) \AA$ & $\beta=109.528(7)^{\circ}$ \\
\hline & $\mathrm{c}=35.660(2) \AA$ & $\gamma=90^{\circ}$ \\
\hline Volume & \multicolumn{2}{|l|}{ 18244.(2) $\AA^{3}$} \\
\hline $\mathbf{Z}$ & \multicolumn{2}{|c|}{8} \\
\hline Density (calculated) & \multicolumn{2}{|l|}{$0.962 \mathrm{~g} / \mathrm{cm}^{3}$} \\
\hline Absorption coefficient & \multicolumn{2}{|l|}{$0.405 \mathrm{~mm}^{-1}$} \\
\hline$F(000)$ & \multicolumn{2}{|l|}{5664} \\
\hline
\end{tabular}

Table S10. Data collection and structure refinement for FR-2.

Theta range for data collection

Index ranges

Reflections collected

Independent reflections

Max. and min. transmission

Refinement method

Refinement program

Function minimized

Data / restraints / parameters

Goodness-of-fit on $\mathbf{F}^{2}$
2.63 to $65.35^{\circ}$

$-53<=\mathrm{h}<=62,-12<=\mathrm{k}<=10,-41<=\mathrm{l}<=41$

42174

$15353[\mathrm{R}(\mathrm{int})=0.0822]$

0.923 and 0.794

Full-matrix least-squares on $\mathrm{F}^{2}$

SHELXL-2014/7 (Sheldrick, 2014)

$\Sigma \mathrm{w}\left(\mathrm{F}_{\mathrm{o}}{ }^{2}-\mathrm{F}_{\mathrm{c}}{ }^{2}\right)^{2}$

$15353 / 0 / 937$

1.577 


\begin{tabular}{|c|c|c|}
\hline \multirow[t]{2}{*}{ Final $\mathbf{R}$ indices } & 9240 data; $\mathrm{I}>2 \sigma(\mathrm{I})$ & $\mathrm{R} 1=0.1757, \mathrm{wR} 2=0.4392$ \\
\hline & all data & $\mathrm{R} 1=0.2139, \mathrm{wR} 2=0.4631$ \\
\hline & \multicolumn{2}{|c|}{$\mathrm{w}=1 /\left[\sigma^{2}\left(\mathrm{~F}_{\mathrm{o}}^{2}\right)+(0.2000 \mathrm{P})^{2}\right]$} \\
\hline weigntung scneme & \multicolumn{2}{|c|}{ where $\mathrm{P}=\left(\mathrm{F}_{\mathrm{o}}^{2}+2 \mathrm{~F}_{\mathrm{c}}^{2}\right) / 3$} \\
\hline Largest diff. peak and hole & \multicolumn{2}{|c|}{0.947 and $-0.514 \mathrm{e}^{-3}$} \\
\hline R.M.S. deviation from mean & \multicolumn{2}{|l|}{$0.133 \mathrm{e}^{-3}$} \\
\hline
\end{tabular}

Table S11. Atomic coordinates and equivalent isotropic atomic displacement parameters $\left(\AA^{2}\right)$ for FR-2.

$U(e q)$ is defined as one third of the trace of the orthogonalized $U_{i j}$ tensor.

$\begin{array}{lcccc} & \mathbf{x} / \mathbf{a} & \mathbf{y} / \mathbf{b} & \mathbf{z} / \mathbf{c} & \mathbf{U}(\mathbf{e q}) \\ \mathrm{C} 1 & 0.11997(11) & 0.7723(7) & 0.04031(15) & 0.0303(14) \\ \mathrm{C} 2 & 0.12051(11) & 0.7345(7) & 0.07996(16) & 0.0321(14) \\ \mathrm{C} 3 & 0.14371(13) & 0.7223(8) & 0.11168(17) & 0.0418(17) \\ \mathrm{C} 4 & 0.14543(12) & 0.6826(7) & 0.14897(17) & 0.0385(16) \\ \mathrm{C} 5 & 0.12186(11) & 0.6492(6) & 0.15611(15) & 0.0292(13) \\ \mathrm{C} 6 & 0.11711(11) & 0.6069(6) & 0.19124(16) & 0.0290(13) \\ \mathrm{C} 7 & 0.13639(12) & 0.5875(7) & 0.23046(15) & 0.0330(15) \\ \mathrm{C} 8 & 0.15791(11) & 0.5021(6) & 0.23758(15) & 0.0253(13) \\ \mathrm{C} 9 & 0.16090(13) & 0.4203(7) & 0.20623(17) & 0.0391(16) \\ \mathrm{C} 10 & 0.18261(14) & 0.3421(8) & 0.21315(19) & 0.0466(18) \\ \mathrm{C} 11 & 0.20135(14) & 0.3295(7) & 0.25110(18) & 0.0392(16) \\ \mathrm{C} 12 & 0.19863(13) & 0.3979(7) & 0.28193(18) & 0.0397(16) \\ \mathrm{C} 13 & 0.17683(11) & 0.4881(6) & 0.27677(15) & 0.0249(13) \\ \mathrm{C} 14 & 0.17431(12) & 0.5571(7) & 0.30846(16) & 0.0325(14) \\ \mathrm{C} 15 & 0.15283(11) & 0.6454(6) & 0.30202(15) & 0.0270(13) \\ \mathrm{C} 16 & 0.14935(12) & 0.7204(7) & 0.33373(17) & 0.0316(14) \\ \mathrm{C} 17 & 0.12958(13) & 0.8104(7) & 0.32748(17) & 0.0395(16) \\ \mathrm{C} 18 & 0.11212(13) & 0.8363(7) & 0.28849(17) & 0.25691(17) \\ \mathrm{C} 19 & 0.11429(12) & 0.7615(7) & 0.26277(16) & 0.0361(15) \\ \mathrm{C} 20 & 0.13385(12) & 0.6656(6) & 14)\end{array}$




\begin{tabular}{|c|c|c|c|c|}
\hline & $\mathbf{x} / \mathbf{a}$ & $\mathbf{y} / \mathbf{b}$ & $\mathbf{z} / \mathbf{c}$ & $\mathrm{U}(\mathbf{e q})$ \\
\hline $\mathrm{C} 21$ & $0.08785(12)$ & $0.5896(7)$ & $0.17963(16)$ & $0.0365(16)$ \\
\hline $\mathrm{C} 22$ & $0.07211(13)$ & $0.5444(8)$ & $0.20163(19)$ & $0.0470(19)$ \\
\hline $\mathrm{C} 23$ & $0.04522(13)$ & $0.5426(9)$ & $0.18370(18)$ & $0.0475(19)$ \\
\hline $\mathrm{C} 24$ & $0.03207(13)$ & $0.5804(8)$ & $0.14499(18)$ & $0.0420(17)$ \\
\hline $\mathrm{C} 25$ & $0.04809(12)$ & $0.6253(8)$ & $0.12136(18)$ & $0.0434(19)$ \\
\hline $\mathrm{C} 26$ & $0.07537(12)$ & $0.6264(7)$ & $0.13881(16)$ & $0.0336(15)$ \\
\hline $\mathrm{C} 27$ & $0.09656(11)$ & $0.6618(7)$ & $0.12304(16)$ & $0.0311(14)$ \\
\hline $\mathrm{C} 28$ & $0.09565(11)$ & $0.7058(6)$ & $0.08691(15)$ & $0.0274(13)$ \\
\hline $\mathrm{C} 29$ & $0.09558(11)$ & $0.8006(7)$ & $0.00865(16)$ & $0.0323(14)$ \\
\hline C30 & $0.09650(11)$ & $0.8396(7)$ & $0.97284(16)$ & $0.0298(14)$ \\
\hline $\mathrm{C} 31$ & $0.07496(13)$ & $0.8749(7)$ & $0.93582(17)$ & $0.0362(15)$ \\
\hline C32 & $0.04735(12)$ & $0.8784(7)$ & $0.92567(16)$ & $0.0348(15)$ \\
\hline $\mathrm{C} 33$ & $0.03122(13)$ & $0.9141(8)$ & $0.88750(18)$ & $0.0427(17)$ \\
\hline C34 & $0.04456(14)$ & $0.9568(8)$ & $0.86057(19)$ & $0.0488(19)$ \\
\hline $\mathrm{C} 35$ & $0.07195(13)$ & $0.9532(7)$ & $0.86961(17)$ & $0.0392(16)$ \\
\hline $\mathrm{C} 36$ & $0.08757(12)$ & $0.9112(7)$ & $0.90802(16)$ & $0.0334(15)$ \\
\hline $\mathrm{C} 37$ & $0.13624(11)$ & $0.9108(7)$ & $0.90491(15)$ & $0.0305(14)$ \\
\hline C38 & $0.13346(12)$ & $0.8366(7)$ & $0.87034(16)$ & $0.0311(14)$ \\
\hline C39 & $0.11427(12)$ & $0.7380(7)$ & $0.85689(17)$ & $0.0396(16)$ \\
\hline $\mathrm{C} 40$ & $0.11186(13)$ & $0.6635(7)$ & $0.82380(17)$ & $0.0396(16)$ \\
\hline $\mathrm{C} 41$ & $0.12940(12)$ & $0.6890(7)$ & $0.80134(16)$ & $0.0329(14)$ \\
\hline $\mathrm{C} 42$ & $0.14875(13)$ & $0.7786(7)$ & $0.81439(17)$ & $0.0382(16)$ \\
\hline $\mathrm{C} 43$ & $0.15264(11)$ & $0.8610(7)$ & $0.84962(16)$ & $0.0300(14)$ \\
\hline C44 & $0.17381(12)$ & $0.9430(6)$ & $0.86456(15)$ & $0.0281(13)$ \\
\hline $\mathrm{C} 45$ & $0.17674(11)$ & $0.0151(6)$ & $0.89967(15)$ & $0.0258(13)$ \\
\hline C46 & $0.19819(13)$ & $0.1040(7)$ & $0.91514(17)$ & $0.0355(15)$ \\
\hline $\mathrm{C} 47$ & $0.20033(15)$ & $0.1796(8)$ & $0.9481(2)$ & $0.0497(19)$ \\
\hline $\mathrm{C} 48$ & $0.18058(13)$ & $0.1668(7)$ & $0.96696(18)$ & $0.0394(16)$ \\
\hline C49 & $0.15969(13)$ & $0.0844(7)$ & $0.95299(18)$ & $0.0378(16)$ \\
\hline C50 & $0.15773(11)$ & $0.0037(6)$ & $0.91982(14)$ & $0.0240(13)$ \\
\hline C51 & $0.11625(11)$ & $0.8942(7)$ & $0.92541(15)$ & $0.0310(14)$ \\
\hline C52 & $0.12134(11)$ & $0.8543(7)$ & $0.96505(15)$ & $0.0304(14)$ \\
\hline
\end{tabular}




\begin{tabular}{|c|c|c|c|c|}
\hline & $\mathbf{x} / \mathbf{a}$ & $\mathbf{y} / \mathbf{b}$ & $\mathbf{z} / \mathbf{c}$ & $\mathrm{U}(\mathbf{e q})$ \\
\hline $\mathrm{C} 53$ & $0.14547(12)$ & $0.8280(7)$ & $0.99570(17)$ & $0.0371(16)$ \\
\hline $\mathrm{C} 54$ & $0.14460(12)$ & $0.7883(8)$ & $0.03222(17)$ & $0.0406(17)$ \\
\hline C55 & $0.00232(12)$ & $0.5890(7)$ & $0.12693(18)$ & $0.0368(15)$ \\
\hline C56 & $0.98612(18)$ & $0.4849(10)$ & $0.1348(2)$ & $0.066(2)$ \\
\hline $\mathrm{C} 57$ & $0.9594(2)$ & $0.4871(11)$ & $0.1199(3)$ & $0.074(3)$ \\
\hline C58 & $0.94602(14)$ & $0.6061(9)$ & $0.0967(2)$ & $0.051(2)$ \\
\hline C59 & $0.96176(16)$ & $0.6991(11)$ & $0.0884(3)$ & $0.074(3)$ \\
\hline $\mathrm{C} 60$ & $0.98976(15)$ & $0.6854(9)$ & $0.1026(2)$ & $0.060(2)$ \\
\hline C61 & $0.19513(11)$ & $0.5454(6)$ & $0.35005(15)$ & $0.0284(13)$ \\
\hline C62 & $0.22068(12)$ & $0.5948(7)$ & $0.35675(17)$ & $0.0373(16)$ \\
\hline C63 & $0.24030(13)$ & $0.5883(7)$ & $0.39338(17)$ & $0.0365(15)$ \\
\hline C64 & $0.23280(13)$ & $0.5274(7)$ & $0.42493(18)$ & $0.0406(16)$ \\
\hline C65 & $0.20781(12)$ & $0.4859(6)$ & $0.41895(15)$ & $0.0289(13)$ \\
\hline C66 & $0.18884(12)$ & $0.4898(6)$ & $0.38080(15)$ & $0.0297(14)$ \\
\hline $\mathrm{C} 67$ & $0.91493(18)$ & $0.6191(11)$ & $0.0792(3)$ & $0.075(3)$ \\
\hline C68 & $0.9055(2)$ & $0.6223(18)$ & $0.1174(4)$ & $0.141(7)$ \\
\hline C69 & $0.90377(17)$ & $0.4948(11)$ & $0.0568(3)$ & $0.072(3)$ \\
\hline $\mathrm{C} 70$ & $0.9064(3)$ & $0.7382(19)$ & $0.0525(5)$ & $0.158(7)$ \\
\hline C71 & $0.26839(14)$ & $0.6415(8)$ & $0.40335(19)$ & $0.0482(19)$ \\
\hline $\mathrm{C} 72$ & $0.27358(16)$ & $0.6822(10)$ & $0.3633(2)$ & $0.067(2)$ \\
\hline $\mathrm{C} 73$ & $0.27106(15)$ & $0.7676(9)$ & $0.4276(2)$ & $0.056(2)$ \\
\hline $\mathrm{C} 74$ & $0.28970(16)$ & $0.5445(10)$ & $0.4251(2)$ & $0.067(2)$ \\
\hline $\mathrm{C} 75$ & $0.20065(13)$ & $0.4193(7)$ & $0.45330(16)$ & $0.0352(15)$ \\
\hline C76 & $0.21761(15)$ & $0.4742(8)$ & $0.49351(17)$ & $0.0502(19)$ \\
\hline $\mathrm{C} 77$ & $0.20632(14)$ & $0.2774(8)$ & $0.4544(2)$ & $0.0494(19)$ \\
\hline $\mathrm{C} 78$ & $0.17127(14)$ & $0.4423(8)$ & $0.4479(2)$ & $0.0486(19)$ \\
\hline C79 & $0.00223(13)$ & $0.9078(8)$ & $0.87515(17)$ & $0.0447(19)$ \\
\hline $\mathrm{C} 80$ & $0.98945(17)$ & $0.8147(12)$ & $0.8877(2)$ & $0.079(3)$ \\
\hline $\mathrm{C} 81$ & $0.96160(19)$ & $0.8026(14)$ & $0.8752(3)$ & $0.094(4)$ \\
\hline $\mathrm{C} 82$ & $0.94586(15)$ & $0.8922(10)$ & $0.8500(2)$ & $0.059(2)$ \\
\hline $\mathrm{C} 83$ & $0.9592(2)$ & $0.0053(12)$ & $0.8403(3)$ & $0.081(3)$ \\
\hline $\mathrm{C} 84$ & $0.98618(19)$ & $0.0108(12)$ & $0.8516(2)$ & $0.076(3)$ \\
\hline
\end{tabular}




\begin{tabular}{lllll} 
& \multicolumn{1}{c}{$\mathbf{x} / \mathbf{a}$} & $\mathbf{y} / \mathbf{b}$ & $\mathbf{z} / \mathbf{c}$ & $\mathbf{U}(\mathbf{e q})$ \\
C85 & $0.91440(17)$ & $0.8826(11)$ & $0.8366(3)$ & $0.072(3)$ \\
C86 & $0.9058(2)$ & $0.7699(15)$ & $0.8550(4)$ & $0.118(5)$ \\
C87 & $0.90349(16)$ & $0.9997(10)$ & $0.8466(2)$ & $0.064(2)$ \\
C88 & $0.9053(2)$ & $0.8770(17)$ & $0.7882(3)$ & $0.135(6)$ \\
C89 & $0.19467(12)$ & $0.9549(7)$ & $0.84450(16)$ & $0.0351(15)$ \\
C90 & $0.18838(12)$ & $0.0094(6)$ & $0.80779(16)$ & $0.0298(14)$ \\
C91 & $0.20786(12)$ & $0.0110(6)$ & $0.78858(16)$ & $0.0290(14)$ \\
C92 & $0.23280(13)$ & $0.9725(7)$ & $0.80767(17)$ & $0.0386(16)$ \\
C93 & $0.24041(14)$ & $0.9134(7)$ & $0.84589(19)$ & $0.0426(17)$ \\
C94 & $0.22037(13)$ & $0.9048(7)$ & $0.86313(17)$ & $0.0360(15)$ \\
C95 & $0.20034(13)$ & $0.0793(7)$ & $0.74612(17)$ & $0.0371(15)$ \\
C96 & $0.17153(14)$ & $0.0494(10)$ & $0.72229(19)$ & $0.055(2)$ \\
C97 & $0.20572(15)$ & $0.2222(8)$ & $0.75131(19)$ & $0.0495(19)$ \\
C98 & $0.21778(16)$ & $0.0120(9)$ & $0.7233(2)$ & $0.059(2)$ \\
C99 & $0.26784(14)$ & $0.8589(8)$ & $0.8646(2)$ & $0.0466(18)$ \\
C100 & $0.29006(18)$ & $0.9582(13)$ & $0.8638(3)$ & $0.088(3)$ \\
C101 & $0.27102(14)$ & $0.7304(8)$ & $0.8440(2)$ & $0.0506(18)$ \\
C102 & $0.27308(16)$ & $0.8220(10)$ & $0.9099(2)$ & $0.067(3)$
\end{tabular}

Table S12. Bond lengths ( $(\AA)$ for FR-2.

$\begin{array}{llll}\text { C1-C29 } & 1.434(8) & \mathrm{C} 1-\mathrm{C} 54 & 1.440(8) \\ \mathrm{C} 1-\mathrm{C} 2 & 1.456(7) & \mathrm{C} 2-\mathrm{C} 3 & 1.372(8) \\ \mathrm{C} 2-\mathrm{C} 28 & 1.452(8) & \mathrm{C} 3-\mathrm{C} 4 & 1.364(8) \\ \mathrm{C} 3-\mathrm{H} 3 & 0.93 & \mathrm{C} 4-\mathrm{C} 5 & 1.400(8) \\ \mathrm{C} 4-\mathrm{H} 4 & 0.93 & \mathrm{C} 5-\mathrm{C} 6 & 1.425(7) \\ \mathrm{C} 5-\mathrm{C} 27 & 1.467(8) & \mathrm{C} 6-\mathrm{C} 7 & 1.445(8) \\ \text { C6-C21 } & 1.479(8) & \mathrm{C} 7-\mathrm{C} 8 & 1.392(8) \\ \text { C7-C20 } & 1.445(8) & \mathrm{C} 8-\mathrm{C} 13 & 1.430(8) \\ \text { C8-C9 } & 1.447(8) & \mathrm{C} 9-\mathrm{C} 10 & 1.356(9) \\ \text { C9-H9 } & 0.93 & \mathrm{C} 10-\mathrm{C} 11 & 1.392(9)\end{array}$




\begin{tabular}{|c|c|c|c|}
\hline C10-H10 & 0.93 & C11-C12 & $1.351(9)$ \\
\hline C11-H11 & 0.93 & C12-C13 & $1.442(8)$ \\
\hline C12-H12 & 0.93 & C13-C14 & $1.377(8)$ \\
\hline C14-C15 & $1.411(8)$ & C14-C61 & $1.530(8)$ \\
\hline C15-C16 & $1.429(8)$ & $\mathrm{C} 15-\mathrm{C} 20$ & $1.440(8)$ \\
\hline C16-C17 & $1.357(9)$ & C16-H16 & 0.93 \\
\hline C17-C18 & $1.415(9)$ & C17-H17 & 0.93 \\
\hline C18-C19 & $1.398(8)$ & C18-H18 & 0.93 \\
\hline C19-C20 & $1.393(9)$ & C19-H19 & 0.93 \\
\hline C21-C22 & $1.402(8)$ & $\mathrm{C} 21-\mathrm{C} 26$ & $1.433(8)$ \\
\hline C22-C23 & $1.356(9)$ & $\mathrm{C} 22-\mathrm{H} 22$ & 0.93 \\
\hline C23-C24 & $1.378(9)$ & $\mathrm{C} 23-\mathrm{H} 23$ & 0.93 \\
\hline C24-C25 & $1.457(8)$ & C24-C55 & $1.495(9)$ \\
\hline $\mathrm{C} 25-\mathrm{C} 26$ & $1.372(8)$ & $\mathrm{C} 25-\mathrm{H} 25$ & 0.93 \\
\hline C26-C27 & $1.461(8)$ & $\mathrm{C} 27-\mathrm{C} 28$ & $1.350(7)$ \\
\hline C28-H28 & 0.93 & C29-C30 & $1.354(8)$ \\
\hline C29-H29 & 0.93 & C30-C52 & $1.444(8)$ \\
\hline C30-C31 & $1.474(8)$ & C31-C32 & $1.388(8)$ \\
\hline C31-C36 & $1.419(8)$ & C32-C33 & $1.393(8)$ \\
\hline C32-H32 & 0.93 & C33-C34 & $1.438(9)$ \\
\hline C33-C79 & $1.454(9)$ & C34-C35 & $1.381(9)$ \\
\hline C34-H34 & 0.93 & C35-C36 & $1.410(8)$ \\
\hline C35-H35 & 0.93 & C36-C51 & $1.450(8)$ \\
\hline C37-C38 & $1.412(8)$ & C37-C50 & $1.443(8)$ \\
\hline C37-C51 & $1.486(7)$ & C38-C39 & $1.399(9)$ \\
\hline C38-C43 & $1.465(7)$ & C39-C40 & $1.374(9)$ \\
\hline C39-H39 & 0.93 & C40-C41 & $1.441(8)$ \\
\hline $\mathrm{C} 40-\mathrm{H} 40$ & 0.93 & C41-C42 & $1.338(9)$ \\
\hline C41-H41 & 0.93 & $\mathrm{C} 42-\mathrm{C} 43$ & $1.469(9)$ \\
\hline C42-H42 & 0.93 & C43-C44 & $1.361(8)$ \\
\hline C44-C45 & $1.416(8)$ & C44-C89 & $1.511(7)$ \\
\hline $\mathrm{C} 45-\mathrm{C} 46$ & $1.416(8)$ & C45-C50 & $1.426(7)$ \\
\hline C46-C47 & $1.378(9)$ & C46-H46 & 0.93 \\
\hline
\end{tabular}




\begin{tabular}{|c|c|c|c|}
\hline C47-C48 & $1.430(9)$ & $\mathrm{C} 47-\mathrm{H} 47$ & 0.93 \\
\hline C48-C49 & $1.348(9)$ & C48-H48 & 0.93 \\
\hline C49-C50 & $1.417(8)$ & C49-H49 & 0.93 \\
\hline C51-C52 & $1.408(7)$ & C52-C53 & $1.404(8)$ \\
\hline C53-C54 & $1.379(8)$ & C53-H53 & 0.93 \\
\hline C54-H54 & 0.93 & C55-C60 & $1.335(11)$ \\
\hline C55-C56 & $1.453(11)$ & C56-C57 & $1.338(12)$ \\
\hline C56-H56 & 0.93 & C57-C58 & $1.509(13)$ \\
\hline C57-H57 & 0.93 & C58-C59 & $1.362(12)$ \\
\hline C58-C67 & $1.563(11)$ & C59-C60 & $1.408(11)$ \\
\hline C59-H59 & 0.93 & C60-H60 & 0.93 \\
\hline C61-C66 & $1.372(8)$ & C61-C62 & $1.392(8)$ \\
\hline C62-C63 & $1.373(9)$ & C62-H62 & 0.93 \\
\hline C63-C64 & $1.453(9)$ & C63-C71 & $1.514(9)$ \\
\hline C64-C65 & $1.340(9)$ & C64-H64 & 0.93 \\
\hline C65-C66 & $1.397(8)$ & C65-C75 & $1.556(7)$ \\
\hline C66-H66 & 0.93 & C67-C69 & $1.513(14)$ \\
\hline C67-C70 & $1.520(19)$ & C67-C68 & $1.599(14)$ \\
\hline C68-H68A & 0.96 & C68-H68B & 0.96 \\
\hline C68-H68C & 0.96 & C69-H69A & 0.96 \\
\hline C69-H69B & 0.96 & C69-H69C & 0.96 \\
\hline C70-H70A & 0.96 & $\mathrm{C} 70-\mathrm{H} 70 \mathrm{~B}$ & 0.96 \\
\hline C70-H70C & 0.96 & C71-C74 & $1.510(12)$ \\
\hline C71-C73 & $1.532(11)$ & C71-C72 & $1.596(10)$ \\
\hline C72-H72A & 0.96 & $\mathrm{C} 72-\mathrm{H} 72 \mathrm{~B}$ & 0.96 \\
\hline C72-H72C & 0.96 & $\mathrm{C} 73-\mathrm{H} 73 \mathrm{~A}$ & 0.96 \\
\hline C73-H73B & 0.96 & $\mathrm{C} 73-\mathrm{H} 73 \mathrm{C}$ & 0.96 \\
\hline C74-H74A & 0.96 & C74-H74B & 0.96 \\
\hline C74-H74C & 0.96 & C75-C77 & $1.479(11)$ \\
\hline C75-C76 & $1.523(9)$ & C75-C78 & $1.525(9)$ \\
\hline C76-H76A & 0.96 & C76-H76B & 0.96 \\
\hline C76-H76C & 0.96 & C77-H77A & 0.96 \\
\hline C77-H77B & 0.96 & $\mathrm{C} 77-\mathrm{H} 77 \mathrm{C}$ & 0.96 \\
\hline
\end{tabular}




\begin{tabular}{|c|c|c|c|}
\hline C78-H78A & 0.96 & C78-H78B & 0.96 \\
\hline C78-H78C & 0.96 & C79-C80 & $1.331(12)$ \\
\hline C79-C84 & $1.436(12)$ & C80-C81 & $1.400(12)$ \\
\hline C80-H80 & 0.93 & C81-C82 & $1.358(14)$ \\
\hline C81-H81 & 0.93 & C82-C83 & $1.458(14)$ \\
\hline $\mathrm{C} 82-\mathrm{C} 85$ & $1.579(11)$ & C83-C84 & $1.352(12)$ \\
\hline $\mathrm{C} 83-\mathrm{H} 83$ & 0.93 & C84-H84 & 0.93 \\
\hline $\mathrm{C} 85-\mathrm{C} 87$ & $1.426(13)$ & C85-C86 & $1.472(17)$ \\
\hline $\mathrm{C} 85-\mathrm{C} 88$ & $1.628(14)$ & C86-H86A & 0.96 \\
\hline C86-H86B & 0.96 & C86-H86C & 0.96 \\
\hline C87-H87A & 0.96 & C87-H87B & 0.96 \\
\hline C87-H87C & 0.96 & C88-H88A & 0.96 \\
\hline C88-H88B & 0.96 & C88-H88C & 0.96 \\
\hline C89-C90 & $1.358(8)$ & C89-C94 & $1.400(9)$ \\
\hline C90-C91 & $1.419(7)$ & C90-H90 & 0.93 \\
\hline C91-C92 & $1.330(9)$ & C91-C95 & $1.592(8)$ \\
\hline C92-C93 & $1.420(9)$ & C92-H92 & 0.93 \\
\hline C93-C94 & $1.398(9)$ & C93-C99 & $1.492(10)$ \\
\hline C94-H94 & 0.93 & C95-C97 & $1.487(11)$ \\
\hline C95-C96 & $1.512(9)$ & C95-C98 & $1.579(9)$ \\
\hline C96-H96A & 0.96 & C96-H96B & 0.96 \\
\hline C96-H96C & 0.96 & C97-H97A & 0.96 \\
\hline C97-H97B & 0.96 & C97-H97C & 0.96 \\
\hline C98-H98A & 0.96 & C98-H98B & 0.96 \\
\hline C98-H98C & 0.96 & C99-C101 & $1.542(11)$ \\
\hline C99-C100 & $1.564(12)$ & C99-C102 & $1.591(10)$ \\
\hline C100-H10A & 0.96 & C100-H10B & 0.96 \\
\hline C100-H10C & 0.96 & C101-H10D & 0.96 \\
\hline C101-H10E & 0.96 & $\mathrm{C} 101-\mathrm{H} 10 \mathrm{~F}$ & 0.96 \\
\hline C102-H10G & 0.96 & $\mathrm{C} 102-\mathrm{H} 10 \mathrm{H}$ & 0.96 \\
\hline C102-H10I & 0.96 & & \\
\hline
\end{tabular}


Table S13. Bond angles $\left({ }^{\circ}\right)$ for FR-2.

$\begin{array}{llll}\text { C29-C1-C54 } & 117.4(5) & \text { C29-C1-C2 } & 122.5(5) \\ \text { C54-C1-C2 } & 120.0(5) & \text { C3-C2-C28 } & 117.2(5) \\ \text { C3-C2-C1 } & 123.1(5) & \text { C28-C2-C1 } & 119.7(5) \\ \text { C4-C3-C2 } & 125.5(6) & \text { C4-C3-H3 } & 117.3 \\ \text { C2-C3-H3 } & 117.3 & \text { C3-C4-C5 } & 118.5(6) \\ \text { C3-C4-H4 } & 120.8 & \text { C5-C4-H4 } & 120.8 \\ \text { C4-C5-C6 } & 131.7(5) & \text { C4-C5-C27 } & 117.8(5) \\ \text { C6-C5-C27 } & 110.5(5) & \text { C5-C6-C7 } & 128.0(5) \\ \text { C5-C6-C21 } & 105.6(5) & \text { C7-C6-C21 } & 126.4(5) \\ \text { C8-C7-C6 } & 122.4(5) & \text { C8-C7-C20 } & 119.4(5) \\ \text { C6-C7-C20 } & 118.1(6) & \text { C7-C8-C13 } & 120.5(5) \\ \text { C7-C8-C9 } & 121.1(5) & \text { C13-C8-C9 } & 118.3(5) \\ \text { C10-C9-C8 } & 120.4(6) & \text { C10-C9-H9 } & 119.8 \\ \text { C8-C9-H9 } & 119.8 & \text { C9-C10-C11 } & 121.3(6) \\ \text { C9-C10-H10 } & 119.4 & \text { C11-C10-H10 } & 119.4 \\ \text { C12-C11-C10 } & 120.5(6) & \text { C12-C11-H11 } & 119.8 \\ \text { C10-C11-H11 } & 119.8 & \text { C11-C12-C13 } & 121.8(6) \\ \text { C11-C12-H12 } & 119.1 & \text { C13-C12-H12 } & 119.1 \\ \text { C14-C13-C8 } & 121.4(5) & \text { C14-C13-C12 } & 121.1(5) \\ \text { C8-C13-C12 } & 117.4(5) & \text { C13-C14-C15 } & 119.3(5) \\ \text { C13-C14-C61 } & 121.2(5) & \text { C15-C14-C61 } & 119.4(5) \\ \text { C14-C15-C16 } & 121.7(5) & \text { C14-C15-C20 } & 121.0(5) \\ \text { C16-C15-C20 } & 117.3(5) & \text { C17-C16-C15 } & 122.1(5) \\ \text { C17-C16-H16 } & 118.9 & \text { C15-C16-H16 } & 118.9 \\ \text { C16-C17-C18 } & 120.3(6) & \text { C16-C17-H17 } & 119.8 \\ \text { C18-C17-H17 } & 119.8 & \text { C19-C18-C17 } & 119.1(6) \\ \text { C19-C18-H18 } & 120.5 & \text { C17-C18-H18 } & 120.5 \\ \text { C20-C19-C18 } & 121.5(6) & \text { C20-C19-H19 } & 119.2 \\ \text { C18-C19-H19 } & 119.2 & 122.2(5) & 119.4(5) \\ \text { C19-C20-C7 } & & 118.3(5) \\ & & & \\ & & & \end{array}$




\begin{tabular}{|c|c|c|c|}
\hline $\mathrm{C} 22-\mathrm{C} 21-\mathrm{C} 26$ & $119.7(6)$ & $\mathrm{C} 22-\mathrm{C} 21-\mathrm{C} 6$ & $130.5(5)$ \\
\hline C26-C21-C6 & $109.8(5)$ & $\mathrm{C} 23-\mathrm{C} 22-\mathrm{C} 21$ & $118.1(6)$ \\
\hline $\mathrm{C} 23-\mathrm{C} 22-\mathrm{H} 22$ & 120.9 & $\mathrm{C} 21-\mathrm{C} 22-\mathrm{H} 22$ & 120.9 \\
\hline $\mathrm{C} 22-\mathrm{C} 23-\mathrm{C} 24$ & $124.7(6)$ & $\mathrm{C} 22-\mathrm{C} 23-\mathrm{H} 23$ & 117.6 \\
\hline $\mathrm{C} 24-\mathrm{C} 23-\mathrm{H} 23$ & 117.6 & $\mathrm{C} 23-\mathrm{C} 24-\mathrm{C} 25$ & $118.0(6)$ \\
\hline C23-C24-C55 & $123.8(5)$ & $\mathrm{C} 25-\mathrm{C} 24-\mathrm{C} 55$ & $118.0(5)$ \\
\hline $\mathrm{C} 26-\mathrm{C} 25-\mathrm{C} 24$ & $118.2(5)$ & $\mathrm{C} 26-\mathrm{C} 25-\mathrm{H} 25$ & 120.9 \\
\hline $\mathrm{C} 24-\mathrm{C} 25-\mathrm{H} 25$ & 120.9 & $\mathrm{C} 25-\mathrm{C} 26-\mathrm{C} 21$ & $121.2(5)$ \\
\hline $\mathrm{C} 25-\mathrm{C} 26-\mathrm{C} 27$ & $131.2(5)$ & $\mathrm{C} 21-\mathrm{C} 26-\mathrm{C} 27$ & $107.6(5)$ \\
\hline $\mathrm{C} 28-\mathrm{C} 27-\mathrm{C} 26$ & $131.6(5)$ & $\mathrm{C} 28-\mathrm{C} 27-\mathrm{C} 5$ & $121.8(5)$ \\
\hline C26-C27-C5 & $106.5(5)$ & $\mathrm{C} 27-\mathrm{C} 28-\mathrm{C} 2$ & $119.1(5)$ \\
\hline $\mathrm{C} 27-\mathrm{C} 28-\mathrm{H} 28$ & 120.5 & $\mathrm{C} 2-\mathrm{C} 28-\mathrm{H} 28$ & 120.5 \\
\hline C30-C29-C1 & $119.6(5)$ & $\mathrm{C} 30-\mathrm{C} 29-\mathrm{H} 29$ & 120.2 \\
\hline C1-C29-H29 & 120.2 & C29-C30-C52 & $122.4(5)$ \\
\hline C29-C30-C31 & $130.9(5)$ & C52-C30-C31 & $106.7(5)$ \\
\hline C32-C31-C36 & $121.0(5)$ & C32-C31-C30 & $132.5(5)$ \\
\hline C36-C31-C30 & $106.5(5)$ & C31-C32-C33 & $120.8(5)$ \\
\hline C31-C32-H32 & 119.6 & C33-C32-H32 & 119.6 \\
\hline C32-C33-C34 & $116.9(6)$ & C32-C33-C79 & $121.8(6)$ \\
\hline C34-C33-C79 & $121.3(6)$ & C35-C34-C33 & $123.5(6)$ \\
\hline C35-C34-H34 & 118.3 & C33-C34-H34 & 118.3 \\
\hline C34-C35-C36 & $117.8(5)$ & C34-C35-H35 & 121.1 \\
\hline C36-C35-H35 & 121.1 & C35-C36-C31 & $119.8(5)$ \\
\hline C35-C36-C51 & $130.1(5)$ & C31-C36-C51 & $110.1(5)$ \\
\hline C38-C37-C50 & $120.9(5)$ & C38-C37-C51 & $119.0(5)$ \\
\hline C50-C37-C51 & $120.0(5)$ & C39-C38-C37 & $122.5(5)$ \\
\hline C39-C38-C43 & $119.9(5)$ & C37-C38-C43 & $117.5(5)$ \\
\hline C40-C39-C38 & $122.8(5)$ & C40-C39-H39 & 118.6 \\
\hline С38-C39-H39 & 118.6 & C39-C40-C41 & $119.0(6)$ \\
\hline C39-C40-H40 & 120.5 & $\mathrm{C} 41-\mathrm{C} 40-\mathrm{H} 40$ & 120.5 \\
\hline $\mathrm{C} 42-\mathrm{C} 41-\mathrm{C} 40$ & $119.6(6)$ & $\mathrm{C} 42-\mathrm{C} 41-\mathrm{H} 41$ & 120.2 \\
\hline C40-C41-H41 & 120.2 & $\mathrm{C} 41-\mathrm{C} 42-\mathrm{C} 43$ & $124.2(5)$ \\
\hline $\mathrm{C} 41-\mathrm{C} 42-\mathrm{H} 42$ & 117.9 & $\mathrm{C} 43-\mathrm{C} 42-\mathrm{H} 42$ & 117.9 \\
\hline
\end{tabular}




\begin{tabular}{|c|c|c|c|}
\hline C44-C43-C38 & $122.0(5)$ & C44-C43-C42 & $123.4(5)$ \\
\hline C38-C43-C42 & $114.3(5)$ & C43-C44-C45 & $120.0(5)$ \\
\hline C43-C44-C89 & $120.3(5)$ & $\mathrm{C} 45-\mathrm{C} 44-\mathrm{C} 89$ & $119.7(5)$ \\
\hline C44-C45-C46 & $121.0(5)$ & $\mathrm{C} 44-\mathrm{C} 45-\mathrm{C} 50$ & $121.1(5)$ \\
\hline $\mathrm{C} 46-\mathrm{C} 45-\mathrm{C} 50$ & $117.9(5)$ & C47-C46-C45 & $121.2(5)$ \\
\hline C47-C46-H46 & 119.4 & $\mathrm{C} 45-\mathrm{C} 46-\mathrm{H} 46$ & 119.4 \\
\hline C46-C47-C48 & 119.0(7) & $\mathrm{C} 46-\mathrm{C} 47-\mathrm{H} 47$ & 120.5 \\
\hline $\mathrm{C} 48-\mathrm{C} 47-\mathrm{H} 47$ & 120.5 & C49-C48-C47 & $121.7(6)$ \\
\hline C49-C48-H48 & 119.1 & C47-C48-H48 & 119.1 \\
\hline C48-C49-C50 & $119.6(5)$ & C48-C49-H49 & 120.2 \\
\hline C50-C49-H49 & 120.2 & C49-C50-C45 & $120.4(5)$ \\
\hline C49-C50-C37 & 121.3(5) & $\mathrm{C} 45-\mathrm{C} 50-\mathrm{C} 37$ & $118.3(5)$ \\
\hline C52-C51-C36 & $106.6(5)$ & C52-C51-C37 & $126.7(5)$ \\
\hline C36-C51-C37 & $126.6(5)$ & C53-C52-C51 & $131.0(5)$ \\
\hline C53-C52-C30 & $119.0(5)$ & C51-C52-C30 & $110.0(5)$ \\
\hline C54-C53-C52 & $118.8(5)$ & C54-C53-H53 & 120.6 \\
\hline C52-C53-H53 & 120.6 & C53-C54-C1 & $122.8(6)$ \\
\hline C53-C54-H54 & 118.6 & C1-C54-H54 & 118.6 \\
\hline C60-C55-C56 & $117.8(7)$ & C60-C55-C24 & $123.3(6)$ \\
\hline C56-C55-C24 & $118.8(7)$ & C57-C56-C55 & $121.9(8)$ \\
\hline C57-C56-H56 & 119.0 & C55-C56-H56 & 119.0 \\
\hline C56-C57-C58 & 118.3(9) & C56-C57-H57 & 120.9 \\
\hline C58-C57-H57 & 120.9 & C59-C58-C57 & 118.1(7) \\
\hline C59-C58-C67 & $119.8(8)$ & C57-C58-C67 & $121.9(8)$ \\
\hline C58-C59-C60 & $120.2(9)$ & C58-C59-H59 & 119.9 \\
\hline C60-C59-H59 & 119.9 & C55-C60-C59 & $123.1(8)$ \\
\hline C55-C60-H60 & 118.5 & C59-C60-H60 & 118.5 \\
\hline C66-C61-C62 & $119.8(5)$ & C66-C61-C14 & $121.2(5)$ \\
\hline C62-C61-C14 & $119.0(5)$ & C63-C62-C61 & $122.1(5)$ \\
\hline C63-C62-H62 & 118.9 & C61-C62-H62 & 118.9 \\
\hline C62-C63-C64 & $116.1(6)$ & C62-C63-C71 & $125.5(6)$ \\
\hline C64-C63-C71 & $118.4(5)$ & C65-C64-C63 & $121.7(6)$ \\
\hline C65-C64-H64 & 119.2 & C63-C64-H64 & 119.2 \\
\hline
\end{tabular}




\begin{tabular}{|c|c|c|c|}
\hline C64-C65-C66 & $119.9(5)$ & C64-C65-C75 & $120.2(5)$ \\
\hline C66-C65-C75 & $119.5(5)$ & C61-C66-C65 & $120.1(6)$ \\
\hline C61-C66-H66 & 119.9 & C65-C66-H66 & 119.9 \\
\hline C69-C67-C70 & $111.0(11)$ & C69-C67-C58 & $108.3(8)$ \\
\hline C70-C67-C58 & $111.8(9)$ & C69-C67-C68 & $106.7(9)$ \\
\hline C70-C67-C68 & $113.9(11)$ & C58-C67-C68 & $104.7(8)$ \\
\hline C67-C68-H68A & 109.5 & C67-C68-H68B & 109.5 \\
\hline H68A-C68-H68B & 109.5 & C67-C68-H68C & 109.5 \\
\hline H68A-C68-H68C & 109.5 & H68B-C68-H68C & 109.5 \\
\hline C67-C69-H69A & 109.5 & C67-C69-H69B & 109.5 \\
\hline H69A-C69-H69B & 109.5 & C67-C69-H69C & 109.5 \\
\hline H69A-C69-H69C & 109.5 & H69B-C69-H69C & 109.5 \\
\hline C67-C70-H70A & 109.5 & C67-C70-H70B & 109.5 \\
\hline H70A-C70-H70B & 109.5 & C67-C70-H70C & 109.5 \\
\hline H70A-C70-H70C & 109.5 & H70B-C70-H70C & 109.5 \\
\hline C74-C71-C63 & $113.3(7)$ & C74-C71-C73 & $110.5(6)$ \\
\hline C63-C71-C73 & $109.4(6)$ & C74-C71-C72 & $107.9(6)$ \\
\hline C63-C71-C72 & $109.6(5)$ & C73-C71-C72 & $105.9(7)$ \\
\hline C71-C72-H72A & 109.5 & C71-C72-H72B & 109.5 \\
\hline H72A-C72-H72B & 109.5 & C71-C72-H72C & 109.5 \\
\hline H72A-C72-H72C & 109.5 & H72B-C72-H72C & 109.5 \\
\hline C71-C73-H73A & 109.5 & C71-C73-H73B & 109.5 \\
\hline H73A-C73-H73B & 109.5 & C71-C73-H73C & 109.5 \\
\hline H73A-C73-H73C & 109.5 & H73B-C73-H73C & 109.5 \\
\hline C71-C74-H74A & 109.5 & C71-C74-H74B & 109.5 \\
\hline H74A-C74-H74B & 109.5 & C71-C74-H74C & 109.5 \\
\hline H74A-C74-H74C & 109.5 & H74B-C74-H74C & 109.5 \\
\hline C77-C75-C76 & $106.5(5)$ & C77-C75-C78 & $110.2(6)$ \\
\hline C76-C75-C78 & $108.7(6)$ & C77-C75-C65 & $110.5(5)$ \\
\hline C76-C75-C65 & $110.9(5)$ & C78-C75-C65 & $110.0(5)$ \\
\hline C75-C76-H76A & 109.5 & C75-C76-H76B & 109.5 \\
\hline H76A-C76-H76B & 109.5 & C75-C76-H76C & 109.5 \\
\hline H76A-C76-H76C & 109.5 & H76B-C76-H76C & 109.5 \\
\hline
\end{tabular}




\begin{tabular}{|c|c|c|c|}
\hline C75-C77-H77A & 109.5 & C75-C77-H77B & 109.5 \\
\hline H77A-C77-H77B & 109.5 & C75-C77-H77C & 109.5 \\
\hline H77A-C77-H77C & 109.5 & H77B-C77-H77C & 109.5 \\
\hline C75-C78-H78A & 109.5 & C75-C78-H78B & 109.5 \\
\hline H78A-C78-H78B & 109.5 & C75-C78-H78C & 109.5 \\
\hline H78A-C78-H78C & 109.5 & H78B-C78-H78C & 109.5 \\
\hline C80-C79-C84 & $116.9(7)$ & C80-C79-C33 & $122.5(7)$ \\
\hline C84-C79-C33 & $120.4(7)$ & C79-C80-C81 & $123.9(9)$ \\
\hline C79-C80-H80 & 118.0 & C81-C80-H80 & 118.0 \\
\hline C82-C81-C80 & $120.2(11)$ & C82-C81-H81 & 119.9 \\
\hline C80-C81-H81 & 119.9 & C81-C82-C83 & $116.8(8)$ \\
\hline C81-C82-C85 & $120.9(9)$ & C83-C82-C85 & $121.8(8)$ \\
\hline C84-C83-C82 & $120.9(9)$ & C84-C83-H83 & 119.6 \\
\hline C82-C83-H83 & 119.6 & C83-C84-C79 & $120.4(10)$ \\
\hline C83-C84-H84 & 119.8 & C79-C84-H84 & 119.8 \\
\hline C87-C85-C86 & $109.4(9)$ & C87-C85-C82 & $110.4(8)$ \\
\hline C86-C85-C82 & 111.6(9) & C87-C85-C88 & $107.0(9)$ \\
\hline C86-C85-C88 & $114.7(10)$ & C82-C85-C88 & $103.4(8)$ \\
\hline C85-C86-H86A & 109.5 & C85-C86-H86B & 109.5 \\
\hline H86A-C86-H86B & 109.5 & C85-C86-H86C & 109.5 \\
\hline H86A-C86-H86C & 109.5 & H86B-C86-H86C & 109.5 \\
\hline C85-C87-H87A & 109.5 & C85-C87-H87B & 109.5 \\
\hline H87A-C87-H87B & 109.5 & C85-C87-H87C & 109.5 \\
\hline H87A-C87-H87C & 109.5 & H87B-C87-H87C & 109.5 \\
\hline C85-C88-H88A & 109.5 & C85-C88-H88B & 109.5 \\
\hline H88A-C88-H88B & 109.5 & $\mathrm{C} 85-\mathrm{C} 88-\mathrm{H} 88 \mathrm{C}$ & 109.5 \\
\hline H88A-C88-H88C & 109.5 & H88B-C88-H88C & 109.5 \\
\hline C90-C89-C94 & $119.6(5)$ & C90-C89-C44 & $120.4(5)$ \\
\hline C94-C89-C44 & $119.9(5)$ & С89-C90-C91 & $119.1(6)$ \\
\hline C89-C90-H90 & 120.5 & C91-C90-H90 & 120.5 \\
\hline C92-C91-C90 & $120.7(5)$ & C92-C91-C95 & $120.5(5)$ \\
\hline C90-C91-C95 & $118.2(5)$ & C91-C92-C93 & $122.2(5)$ \\
\hline C91-C92-H92 & 118.9 & C93-C92-H92 & 118.9 \\
\hline
\end{tabular}




\begin{tabular}{|c|c|c|c|}
\hline С94-C93-C92 & $115.7(6)$ & C94-C93-C99 & $123.6(6)$ \\
\hline C92-C93-C99 & $120.6(6)$ & C93-C94-C89 & $122.2(6)$ \\
\hline C93-C94-H94 & 118.9 & C89-C94-H94 & 118.9 \\
\hline C97-C95-C96 & $112.6(7)$ & C97-C95-C98 & $111.8(6)$ \\
\hline C96-C95-C98 & $106.2(6)$ & C97-C95-C91 & $109.6(5)$ \\
\hline С96-C95-C91 & $109.3(5)$ & C98-C95-C91 & $107.1(5)$ \\
\hline C95-C96-H96A & 109.5 & C95-C96-H96B & 109.5 \\
\hline H96А-С96-H96B & 109.5 & C95-C96-H96C & 109.5 \\
\hline H96A-C96-H96C & 109.5 & H96B-C96-H96C & 109.5 \\
\hline C95-C97-H97A & 109.5 & С95-C97-H97B & 109.5 \\
\hline H97A-C97-H97B & 109.5 & C95-C97-H97C & 109.5 \\
\hline H97A-C97-H97C & 109.5 & H97B-C97-H97C & 109.5 \\
\hline C95-C98-H98A & 109.5 & С95-C98-H98B & 109.5 \\
\hline H98A-C98-H98B & 109.5 & C95-C98-H98C & 109.5 \\
\hline H98A-C98-H98C & 109.5 & H98B-C98-H98C & 109.5 \\
\hline C93-C99-C101 & $110.6(6)$ & C93-С99-C100 & $112.3(7)$ \\
\hline C101-C99-C100 & $109.9(6)$ & C93-C99-C102 & $110.8(5)$ \\
\hline C101-C99-C102 & $105.8(7)$ & C100-C99-C102 & $107.2(7)$ \\
\hline C99-C100-H10A & 109.5 & C99-C100-H10B & 109.5 \\
\hline H10A-C100-H10B & 109.5 & C99-C100-H10C & 109.5 \\
\hline H10A-C100-H10C & 109.5 & H10B-C100-H10C & 109.5 \\
\hline C99-C101-H10D & 109.5 & C99-C101-H10E & 109.5 \\
\hline H10D-C101-H10E & 109.5 & C99-C101-H10F & 109.5 \\
\hline H10D-C101-H10F & 109.5 & H10E-C101-H10F & 109.5 \\
\hline C99-C102-H10G & 109.5 & C99-C102-H10H & 109.5 \\
\hline H10G-C102-H10H & 109.5 & C99-C102-H10I & 109.5 \\
\hline H10G-C102-H10I & 109.5 & H10H-C102-H10I & 109.5 \\
\hline
\end{tabular}

Table S14. Torsion angles $\left({ }^{\circ}\right)$ for FR-2.

$\begin{array}{llll}\mathrm{C} 29-\mathrm{C} 1-\mathrm{C} 2-\mathrm{C} 3 & -172.0(7) & \mathrm{C} 54-\mathrm{C} 1-\mathrm{C} 2-\mathrm{C} 3 & 5.1(11) \\ \mathrm{C} 29-\mathrm{C} 1-\mathrm{C} 2-\mathrm{C} 28 & 9.0(10) & \mathrm{C} 54-\mathrm{C} 1-\mathrm{C} 2-\mathrm{C} 28 & -173.9(6)\end{array}$




\begin{tabular}{|c|c|c|c|}
\hline $\mathrm{C} 28-\mathrm{C} 2-\mathrm{C} 3-\mathrm{C} 4$ & $1.6(12)$ & $\mathrm{C} 1-\mathrm{C} 2-\mathrm{C} 3-\mathrm{C} 4$ & $-177.4(7)$ \\
\hline $\mathrm{C} 2-\mathrm{C} 3-\mathrm{C} 4-\mathrm{C} 5$ & $0.9(12)$ & $\mathrm{C} 3-\mathrm{C} 4-\mathrm{C} 5-\mathrm{C} 6$ & $-178.9(7)$ \\
\hline $\mathrm{C} 3-\mathrm{C} 4-\mathrm{C} 5-\mathrm{C} 27$ & $-1.3(10)$ & $\mathrm{C} 4-\mathrm{C} 5-\mathrm{C} 6-\mathrm{C} 7$ & $0.4(12)$ \\
\hline $\mathrm{C} 27-\mathrm{C} 5-\mathrm{C} 6-\mathrm{C} 7$ & $-177.4(6)$ & $\mathrm{C} 4-\mathrm{C} 5-\mathrm{C} 6-\mathrm{C} 21$ & $179.0(7)$ \\
\hline C27-C5-C6-C21 & $1.2(7)$ & $\mathrm{C} 5-\mathrm{C} 6-\mathrm{C} 7-\mathrm{C} 8$ & $-57.7(10)$ \\
\hline C21-C6-C7-C8 & $124.0(7)$ & $\mathrm{C} 5-\mathrm{C} 6-\mathrm{C} 7-\mathrm{C} 20$ & $118.8(7)$ \\
\hline C21-C6-C7-C20 & $-59.6(9)$ & C6-C7-C8-C13 & $178.7(6)$ \\
\hline C20-C7-C8-C13 & $2.3(9)$ & C6-C7-C8-C9 & $-4.9(10)$ \\
\hline C20-C7-C8-C9 & $178.7(6)$ & C7-C8-C9-C10 & $177.0(7)$ \\
\hline C13-C8-C9-C10 & $-6.5(10)$ & C8-C9-C10-C11 & $5.6(11)$ \\
\hline C9-C10-C11-C12 & $-1.6(11)$ & $\mathrm{C} 10-\mathrm{C} 11-\mathrm{C} 12-\mathrm{C} 13$ & $-1.5(11)$ \\
\hline C7-C8-C13-C14 & $0.4(9)$ & C9-C8-C13-C14 & $-176.1(6)$ \\
\hline C7-C8-C13-C12 & $-180.0(6)$ & C9-C8-C13-C12 & $3.5(8)$ \\
\hline C11-C12-C13-C14 & $180.0(6)$ & $\mathrm{C} 11-\mathrm{C} 12-\mathrm{C} 13-\mathrm{C} 8$ & $0.4(10)$ \\
\hline C8-C13-C14-C15 & $-1.1(9)$ & C12-C13-C14-C15 & $179.3(6)$ \\
\hline C8-C13-C14-C61 & $-177.8(5)$ & C12-C13-C14-C61 & $2.6(9)$ \\
\hline C13-C14-C15-C16 & $-179.5(6)$ & C61-C14-C15-C16 & $-2.7(9)$ \\
\hline C13-C14-C15-C20 & $-1.0(9)$ & C61-C14-C15-C20 & $175.8(5)$ \\
\hline C14-C15-C16-C17 & $176.5(6)$ & $\mathrm{C} 20-\mathrm{C} 15-\mathrm{C} 16-\mathrm{C} 17$ & $-2.0(9)$ \\
\hline C15-C16-C17-C18 & $-2.3(10)$ & C16-C17-C18-C19 & $4.6(10)$ \\
\hline C17-C18-C19-C20 & $-2.4(10)$ & C18-C19-C20-C15 & $-1.9(10)$ \\
\hline C18-C19-C20-C7 & $-179.9(6)$ & C14-C15-C20-C19 & $-174.5(6)$ \\
\hline C16-C15-C20-C19 & $4.1(9)$ & C14-C15-C20-C7 & $3.6(9)$ \\
\hline C16-C15-C20-C7 & $-177.8(5)$ & C8-C7-C20-C19 & $173.8(6)$ \\
\hline C6-C7-C20-C19 & $-2.8(9)$ & C8-C7-C20-C15 & $-4.2(9)$ \\
\hline C6-C7-C20-C15 & $179.3(5)$ & C5-C6-C21-C22 & $177.0(8)$ \\
\hline C7-C6-C21-C22 & $-4.3(12)$ & C5-C6-C21-C26 & $-2.5(7)$ \\
\hline C7-C6-C21-C26 & $176.2(6)$ & $\mathrm{C} 26-\mathrm{C} 21-\mathrm{C} 22-\mathrm{C} 23$ & $-2.8(11)$ \\
\hline C6-C21-C22-C23 & $177.7(7)$ & $\mathrm{C} 21-\mathrm{C} 22-\mathrm{C} 23-\mathrm{C} 24$ & $0.9(13)$ \\
\hline $\mathrm{C} 22-\mathrm{C} 23-\mathrm{C} 24-\mathrm{C} 25$ & $0.2(13)$ & C22-C23-C24-C55 & $-174.3(8)$ \\
\hline $\mathrm{C} 23-\mathrm{C} 24-\mathrm{C} 25-\mathrm{C} 26$ & $0.6(11)$ & $\mathrm{C} 55-\mathrm{C} 24-\mathrm{C} 25-\mathrm{C} 26$ & $175.4(7)$ \\
\hline $\mathrm{C} 24-\mathrm{C} 25-\mathrm{C} 26-\mathrm{C} 21$ & $-2.5(11)$ & $\mathrm{C} 24-\mathrm{C} 25-\mathrm{C} 26-\mathrm{C} 27$ & $178.1(7)$ \\
\hline $\mathrm{C} 22-\mathrm{C} 21-\mathrm{C} 26-\mathrm{C} 25$ & $3.7(11)$ & C6-C21-C26-C25 & $-176.8(7)$ \\
\hline
\end{tabular}




\begin{tabular}{|c|c|c|c|}
\hline $\mathrm{C} 22-\mathrm{C} 21-\mathrm{C} 26-\mathrm{C} 27$ & $-176.8(7)$ & $\mathrm{C} 6-\mathrm{C} 21-\mathrm{C} 26-\mathrm{C} 27$ & $2.8(8)$ \\
\hline $\mathrm{C} 25-\mathrm{C} 26-\mathrm{C} 27-\mathrm{C} 28$ & $1.3(13)$ & $\mathrm{C} 21-\mathrm{C} 26-\mathrm{C} 27-\mathrm{C} 28$ & $-178.2(7)$ \\
\hline $\mathrm{C} 25-\mathrm{C} 26-\mathrm{C} 27-\mathrm{C} 5$ & $177.5(7)$ & $\mathrm{C} 21-\mathrm{C} 26-\mathrm{C} 27-\mathrm{C} 5$ & $-2.0(8)$ \\
\hline $\mathrm{C} 4-\mathrm{C} 5-\mathrm{C} 27-\mathrm{C} 28$ & $-1.0(10)$ & C6-C5-C27-C28 & $177.2(6)$ \\
\hline $\mathrm{C} 4-\mathrm{C} 5-\mathrm{C} 27-\mathrm{C} 26$ & $-177.7(6)$ & C6-C5-C27-C26 & $0.4(8)$ \\
\hline $\mathrm{C} 26-\mathrm{C} 27-\mathrm{C} 28-\mathrm{C} 2$ & $179.3(7)$ & $\mathrm{C} 5-\mathrm{C} 27-\mathrm{C} 28-\mathrm{C} 2$ & $3.5(10)$ \\
\hline $\mathrm{C} 3-\mathrm{C} 2-\mathrm{C} 28-\mathrm{C} 27$ & $-3.8(10)$ & $\mathrm{C} 1-\mathrm{C} 2-\mathrm{C} 28-\mathrm{C} 27$ & $175.2(6)$ \\
\hline C54-C1-C29-C30 & $0.5(10)$ & $\mathrm{C} 2-\mathrm{C} 1-\mathrm{C} 29-\mathrm{C} 30$ & $177.7(6)$ \\
\hline C1-C29-C30-C52 & $0.0(10)$ & C1-C29-C30-C31 & $-179.7(7)$ \\
\hline C29-C30-C31-C32 & $-0.8(13)$ & C52-C30-C31-C32 & $179.5(7)$ \\
\hline C29-C30-C31-C36 & $178.7(7)$ & C52-C30-C31-C36 & $-1.1(8)$ \\
\hline C36-C31-C32-C33 & $1.8(11)$ & C30-C31-C32-C33 & $-178.8(8)$ \\
\hline C31-C32-C33-C34 & $-4.9(11)$ & C31-C32-C33-C79 & $174.7(7)$ \\
\hline C32-C33-C34-C35 & $5.6(12)$ & C79-C33-C34-C35 & $-173.9(8)$ \\
\hline C33-C34-C35-C36 & $-3.0(12)$ & C34-C35-C36-C31 & $-0.3(11)$ \\
\hline C34-C35-C36-C51 & $178.2(7)$ & C32-C31-C36-C35 & $0.9(11)$ \\
\hline C30-C31-C36-C35 & $-178.6(6)$ & C32-C31-C36-C51 & $-177.8(6)$ \\
\hline C30-C31-C36-C51 & $2.7(8)$ & C50-C37-C38-C39 & $174.6(6)$ \\
\hline C51-C37-C38-C39 & $-6.9(10)$ & $\mathrm{C} 50-\mathrm{C} 37-\mathrm{C} 38-\mathrm{C} 43$ & $-2.0(9)$ \\
\hline C51-C37-C38-C43 & $176.6(5)$ & C37-C38-C39-C40 & $-178.5(7)$ \\
\hline C43-C38-C39-C40 & $-2.0(10)$ & C38-C39-C40-C41 & $-1.3(11)$ \\
\hline C39-C40-C41-C42 & $4.3(10)$ & $\mathrm{C} 40-\mathrm{C} 41-\mathrm{C} 42-\mathrm{C} 43$ & $-3.9(10)$ \\
\hline C39-C38-C43-C44 & $-171.0(6)$ & C37-C38-C43-C44 & $5.7(9)$ \\
\hline C39-C38-C43-C42 & $2.3(9)$ & C37-C38-C43-C42 & $179.0(6)$ \\
\hline C41-C42-C43-C44 & $173.8(6)$ & C41-C42-C43-C38 & $0.6(9)$ \\
\hline C38-C43-C44-C45 & $-4.6(9)$ & $\mathrm{C} 42-\mathrm{C} 43-\mathrm{C} 44-\mathrm{C} 45$ & $-177.3(6)$ \\
\hline C38-C43-C44-C89 & $173.7(6)$ & C42-C43-C44-C89 & $1.0(9)$ \\
\hline C43-C44-C45-C46 & $-178.1(6)$ & C89-C44-C45-C46 & $3.5(9)$ \\
\hline C43-C44-C45-C50 & $-0.2(9)$ & C89-C44-C45-C50 & $-178.5(6)$ \\
\hline C44-C45-C46-C47 & $176.4(7)$ & $\mathrm{C} 50-\mathrm{C} 45-\mathrm{C} 46-\mathrm{C} 47$ & $-1.5(10)$ \\
\hline C45-C46-C47-C48 & $0.5(11)$ & $\mathrm{C} 46-\mathrm{C} 47-\mathrm{C} 48-\mathrm{C} 49$ & $-1.2(11)$ \\
\hline C47-C48-C49-C50 & $2.9(11)$ & C48-C49-C50-C45 & $-4.0(10)$ \\
\hline C48-C49-C50-C37 & $177.6(6)$ & C44-C45-C50-C49 & $-174.7(6)$ \\
\hline
\end{tabular}




\begin{tabular}{|c|c|c|c|}
\hline C46-C45-C50-C49 & $3.2(9)$ & C44-C45-C50-C37 & $3.7(9)$ \\
\hline C46-C45-C50-C37 & $-178.3(6)$ & C38-C37-C50-C49 & $176.0(6)$ \\
\hline C51-C37-C50-C49 & $-2.6(9)$ & C38-C37-C50-C45 & $-2.5(9)$ \\
\hline C51-C37-C50-C45 & $179.0(5)$ & C35-C36-C51-C52 & $178.1(7)$ \\
\hline C31-C36-C51-C52 & $-3.3(8)$ & C35-C36-C51-C37 & $-4.1(12)$ \\
\hline C31-C36-C51-C37 & $174.5(7)$ & C38-C37-C51-C52 & $120.8(7)$ \\
\hline C50-C37-C51-C52 & $-60.6(10)$ & C38-C37-C51-C36 & $-56.5(10)$ \\
\hline C50-C37-C51-C36 & $122.0(7)$ & C36-C51-C52-C53 & $-177.8(7)$ \\
\hline C37-C51-C52-C53 & $4.4(12)$ & C36-C51-C52-C30 & $2.6(8)$ \\
\hline C37-C51-C52-C30 & $-175.2(6)$ & C29-C30-C52-C53 & $-0.4(10)$ \\
\hline C31-C30-C52-C53 & $179.3(6)$ & C29-C30-C52-C51 & $179.3(6)$ \\
\hline C31-C30-C52-C51 & $-1.0(8)$ & C51-C52-C53-C54 & $-179.3(7)$ \\
\hline C30-C52-C53-C54 & $0.4(10)$ & C52-C53-C54-C1 & $0.1(11)$ \\
\hline C29-C1-C54-C53 & $-0.6(11)$ & C2-C1-C54-C53 & $-177.8(7)$ \\
\hline C23-C24-C55-C60 & $139.5(9)$ & C25-C24-C55-C60 & $-35.0(11)$ \\
\hline C23-C24-C55-C56 & $-43.1(11)$ & C25-C24-C55-C56 & $142.5(7)$ \\
\hline C60-C55-C56-C57 & $-3.0(12)$ & C24-C55-C56-C57 & $179.4(7)$ \\
\hline C55-C56-C57-C58 & $-4.3(12)$ & C56-C57-C58-C59 & $6.9(12)$ \\
\hline C56-C57-C58-C67 & $-178.1(8)$ & C57-C58-C59-C60 & $-2.4(13)$ \\
\hline C67-C58-C59-C60 & $-177.4(8)$ & C56-C55-C60-C59 & $8.0(12)$ \\
\hline C24-C55-C60-C59 & $-174.5(8)$ & C58-C59-C60-C55 & $-5.3(14)$ \\
\hline C13-C14-C61-C66 & $-113.5(7)$ & C15-C14-C61-C66 & $69.8(8)$ \\
\hline C13-C14-C61-C62 & $67.9(9)$ & C15-C14-C61-C62 & $-108.9(7)$ \\
\hline C66-C61-C62-C63 & $1.0(10)$ & C14-C61-C62-C63 & $179.6(6)$ \\
\hline C61-C62-C63-C64 & $-0.3(10)$ & C61-C62-C63-C71 & $-178.9(7)$ \\
\hline C62-C63-C64-C65 & $-3.1(10)$ & C71-C63-C64-C65 & $175.5(7)$ \\
\hline C63-C64-C65-C66 & $5.9(10)$ & C63-C64-C65-C75 & 179.1(6) \\
\hline C62-C61-C66-C65 & $1.7(9)$ & C14-C61-C66-C65 & $-176.9(6)$ \\
\hline C64-C65-C66-C61 & $-5.2(10)$ & C75-C65-C66-C61 & $-178.4(6)$ \\
\hline C59-C58-C67-C69 & $125.3(9)$ & C57-C58-C67-C69 & $-49.5(10)$ \\
\hline C59-C58-C67-C70 & $2.7(14)$ & C57-C58-C67-C70 & $-172.1(10)$ \\
\hline C59-C58-C67-C68 & $-121.1(11)$ & C57-C58-C67-C68 & $64.1(11)$ \\
\hline C62-C63-C71-C74 & $-131.1(7)$ & C64-C63-C71-C74 & $50.4(8)$ \\
\hline
\end{tabular}




\begin{tabular}{|c|c|c|c|}
\hline C62-C63-C71-C73 & $105.1(8)$ & C64-C63-C71-C73 & $-73.4(8)$ \\
\hline C62-C63-C71-C72 & $-10.5(10)$ & C64-C63-C71-C72 & $170.9(7)$ \\
\hline C64-C65-C75-C77 & $-86.3(8)$ & C66-C65-C75-C77 & $86.9(7)$ \\
\hline C64-C65-C75-C76 & $31.6(8)$ & C66-C65-C75-C76 & $-155.2(6)$ \\
\hline C64-C65-C75-C78 & $151.8(7)$ & C66-C65-C75-C78 & $-35.0(8)$ \\
\hline C32-C33-C79-C80 & $-35.5(12)$ & C34-C33-C79-C80 & $144.0(9)$ \\
\hline C32-C33-C79-C84 & $138.3(8)$ & C34-C33-C79-C84 & $-42.2(11)$ \\
\hline C84-C79-C80-C81 & $8.7(14)$ & C33-C79-C80-C81 & $-177.3(9)$ \\
\hline C79-C80-C81-C82 & $-2.9(16)$ & C80-C81-C82-C83 & $-5.8(14)$ \\
\hline C80-C81-C82-C85 & $-178.2(9)$ & C81-C82-C83-C84 & $8.5(14)$ \\
\hline C85-C82-C83-C84 & $-179.1(8)$ & C82-C83-C84-C79 & $-2.9(14)$ \\
\hline C80-C79-C84-C83 & $-5.6(13)$ & C33-C79-C84-C83 & $-179.7(8)$ \\
\hline C81-C82-C85-C87 & $122.6(10)$ & C83-C82-C85-C87 & $-49.4(11)$ \\
\hline C81-C82-C85-C86 & $0.7(13)$ & C83-C82-C85-C86 & $-171.3(9)$ \\
\hline C81-C82-C85-C88 & $-123.1(11)$ & C83-C82-C85-C88 & $64.8(12)$ \\
\hline C43-C44-C89-C90 & $67.5(9)$ & C45-C44-C89-C90 & $-114.2(7)$ \\
\hline C43-C44-C89-C94 & $-110.0(7)$ & C45-C44-C89-C94 & $68.4(9)$ \\
\hline C94-C89-C90-C91 & $1.8(10)$ & C44-C89-C90-C91 & $-175.7(6)$ \\
\hline C89-C90-С91-С92 & $-7.0(10)$ & C89-C90-C91-C95 & $-178.2(6)$ \\
\hline С90-С91-С92-С93 & $7.9(10)$ & C95-C91-C92-C93 & $178.8(6)$ \\
\hline C91-C92-C93-C94 & $-3.4(10)$ & C91-C92-C93-C99 & $173.6(7)$ \\
\hline С92-C93-С94-С89 & $-1.9(10)$ & C99-С93-C94-C89 & $-178.8(7)$ \\
\hline C90-C89-C94-C93 & $2.6(10)$ & C44-C89-C94-C93 & $-179.9(6)$ \\
\hline C92-C91-C95-C97 & $-85.8(8)$ & C90-C91-C95-C97 & $85.4(7)$ \\
\hline C92-C91-C95-C96 & $150.4(7)$ & C90-C91-C95-C96 & $-38.5(8)$ \\
\hline C92-C91-C95-C98 & $35.7(8)$ & C90-C91-C95-C98 & $-153.2(6)$ \\
\hline C94-C93-C99-C101 & $103.5(8)$ & C92-C93-C99-C101 & $-73.2(9)$ \\
\hline C94-C93-C99-C100 & $-133.3(8)$ & C92-C93-C99-C100 & 49.9(9) \\
\hline С94-C93-C99-C102 & $-13.4(10)$ & C92-C93-C99-C102 & $169.8(7)$ \\
\hline
\end{tabular}


Table S15. Anisotropic atomic displacement parameters $\left(\AA^{2}\right)$ for FR-2.

The anisotropic atomic displacement factor exponent takes the form: $-2 \pi^{2}\left[h^{2} a^{* 2} U_{11}+\ldots+2 h k a^{*} b^{*} U_{12}\right]$

\begin{tabular}{|c|c|c|c|c|c|c|}
\hline & $\mathbf{U}_{11}$ & $\mathbf{U}_{22}$ & $\mathbf{U}_{33}$ & $\mathbf{U}_{23}$ & $\mathbf{U}_{13}$ & $\mathbf{U}_{12}$ \\
\hline $\mathrm{C} 1$ & $0.032(3)$ & $0.046(4)$ & $0.017(3)$ & $0.010(2)$ & $0.013(2)$ & $0.000(3)$ \\
\hline $\mathrm{C} 2$ & $0.033(3)$ & $0.047(4)$ & $0.022(3)$ & $0.009(3)$ & $0.016(2)$ & $0.000(3)$ \\
\hline $\mathrm{C} 3$ & $0.039(4)$ & $0.064(5)$ & $0.023(3)$ & $0.000(3)$ & $0.010(3)$ & $-0.010(3)$ \\
\hline $\mathrm{C} 4$ & $0.036(4)$ & $0.061(5)$ & $0.018(3)$ & $0.002(3)$ & $0.010(2)$ & $0.008(3)$ \\
\hline $\mathrm{C} 5$ & $0.036(3)$ & $0.036(4)$ & $0.018(3)$ & $0.003(2)$ & $0.012(2)$ & $-0.003(3)$ \\
\hline C6 & $0.036(3)$ & $0.033(4)$ & $0.021(3)$ & $0.003(2)$ & $0.014(2)$ & $0.003(3)$ \\
\hline $\mathrm{C} 7$ & $0.043(4)$ & $0.043(4)$ & $0.015(3)$ & $0.003(2)$ & $0.013(2)$ & $0.003(3)$ \\
\hline $\mathrm{C} 8$ & $0.032(3)$ & $0.030(4)$ & $0.016(3)$ & $-0.006(2)$ & $0.010(2)$ & $0.005(2)$ \\
\hline C9 & $0.047(4)$ & $0.048(5)$ & $0.022(3)$ & $0.003(3)$ & $0.012(3)$ & $0.007(3)$ \\
\hline $\mathrm{C} 10$ & $0.052(4)$ & $0.057(5)$ & $0.033(4)$ & $-0.004(3)$ & $0.018(3)$ & $0.005(4)$ \\
\hline $\mathrm{C} 11$ & $0.052(4)$ & $0.034(4)$ & $0.032(3)$ & $0.002(3)$ & $0.014(3)$ & $0.008(3)$ \\
\hline $\mathrm{C} 12$ & $0.048(4)$ & $0.049(5)$ & $0.025(3)$ & $0.005(3)$ & $0.015(3)$ & $0.014(3)$ \\
\hline C13 & $0.037(3)$ & $0.025(3)$ & $0.019(3)$ & $0.005(2)$ & $0.016(2)$ & $0.000(2)$ \\
\hline $\mathrm{C} 14$ & $0.040(4)$ & $0.042(4)$ & $0.019(3)$ & $0.001(3)$ & $0.015(2)$ & $0.000(3)$ \\
\hline $\mathrm{C} 15$ & $0.039(3)$ & $0.030(4)$ & $0.013(3)$ & $0.001(2)$ & $0.009(2)$ & $0.002(3)$ \\
\hline C16 & $0.036(3)$ & $0.038(4)$ & $0.025(3)$ & $0.001(3)$ & $0.016(2)$ & $-0.001(3)$ \\
\hline $\mathrm{C} 17$ & $0.045(4)$ & $0.054(5)$ & $0.027(3)$ & $-0.005(3)$ & $0.023(3)$ & $-0.003(3)$ \\
\hline $\mathrm{C} 18$ & $0.046(4)$ & $0.046(5)$ & $0.031(3)$ & $-0.012(3)$ & $0.025(3)$ & $0.004(3)$ \\
\hline C19 & $0.042(4)$ & $0.047(5)$ & $0.021(3)$ & $0.004(3)$ & $0.013(3)$ & $0.009(3)$ \\
\hline $\mathrm{C} 20$ & $0.039(3)$ & $0.033(4)$ & $0.027(3)$ & $-0.003(2)$ & $0.019(3)$ & $-0.003(3)$ \\
\hline $\mathrm{C} 21$ & $0.036(4)$ & $0.059(5)$ & $0.016(3)$ & $0.003(3)$ & $0.010(2)$ & $0.004(3)$ \\
\hline $\mathrm{C} 22$ & $0.043(4)$ & $0.073(6)$ & $0.030(3)$ & $0.012(3)$ & $0.019(3)$ & $0.005(4)$ \\
\hline $\mathrm{C} 23$ & $0.039(4)$ & $0.079(6)$ & $0.030(3)$ & $0.002(3)$ & $0.020(3)$ & $-0.006(4)$ \\
\hline $\mathrm{C} 24$ & $0.038(4)$ & $0.064(5)$ & $0.032(3)$ & $0.014(3)$ & $0.022(3)$ & $0.008(3)$ \\
\hline $\mathrm{C} 25$ & $0.029(4)$ & $0.079(6)$ & $0.026(3)$ & $0.019(3)$ & $0.014(3)$ & $0.004(3)$ \\
\hline $\mathrm{C} 26$ & $0.045(4)$ & $0.042(4)$ & $0.023(3)$ & $0.001(3)$ & $0.024(3)$ & $-0.006(3)$ \\
\hline $\mathrm{C} 27$ & $0.025(3)$ & $0.048(4)$ & $0.022(3)$ & $0.008(3)$ & $0.010(2)$ & $0.001(3)$ \\
\hline
\end{tabular}




\begin{tabular}{|c|c|c|c|c|c|c|}
\hline & $\mathbf{U}_{11}$ & $\mathbf{U}_{22}$ & $\mathbf{U}_{33}$ & $\mathbf{U}_{23}$ & $\mathbf{U}_{13}$ & $\mathbf{U}_{12}$ \\
\hline $\mathrm{C} 28$ & $0.030(3)$ & $0.039(4)$ & $0.019(3)$ & $0.002(2)$ & $0.016(2)$ & $0.000(3)$ \\
\hline $\mathrm{C} 29$ & $0.028(3)$ & $0.047(4)$ & $0.021(3)$ & $0.004(3)$ & $0.007(2)$ & $0.003(3)$ \\
\hline $\mathrm{C} 30$ & $0.030(3)$ & $0.042(4)$ & $0.020(3)$ & $0.004(2)$ & $0.012(2)$ & $0.001(3)$ \\
\hline C31 & $0.043(4)$ & $0.042(4)$ & $0.026(3)$ & $0.004(3)$ & $0.014(3)$ & $-0.006(3)$ \\
\hline $\mathrm{C} 32$ & $0.033(3)$ & $0.052(5)$ & $0.021(3)$ & $0.005(3)$ & $0.012(2)$ & $0.003(3)$ \\
\hline C33 & $0.038(4)$ & $0.059(5)$ & $0.030(3)$ & $0.007(3)$ & $0.009(3)$ & $0.000(3)$ \\
\hline C34 & $0.054(5)$ & $0.070(6)$ & $0.025(3)$ & $0.011(3)$ & $0.016(3)$ & $0.009(4)$ \\
\hline $\mathrm{C} 35$ & $0.043(4)$ & $0.052(5)$ & $0.024(3)$ & $0.005(3)$ & $0.013(3)$ & $-0.002(3)$ \\
\hline C36 & $0.033(3)$ & $0.050(4)$ & $0.020(3)$ & $0.005(3)$ & $0.012(2)$ & $-0.005(3)$ \\
\hline $\mathrm{C} 37$ & $0.035(3)$ & $0.046(4)$ & $0.013(3)$ & $0.001(2)$ & $0.013(2)$ & $-0.005(3)$ \\
\hline C38 & $0.041(4)$ & $0.037(4)$ & $0.023(3)$ & $0.003(2)$ & $0.021(3)$ & $-0.003(3)$ \\
\hline C39 & $0.042(4)$ & $0.054(5)$ & $0.029(3)$ & $-0.010(3)$ & $0.020(3)$ & $-0.012(3)$ \\
\hline $\mathrm{C} 40$ & $0.044(4)$ & $0.052(5)$ & $0.025(3)$ & $-0.007(3)$ & $0.014(3)$ & $-0.011(3)$ \\
\hline $\mathrm{C} 41$ & $0.040(4)$ & $0.041(4)$ & $0.021(3)$ & $-0.003(2)$ & $0.015(3)$ & $-0.003(3)$ \\
\hline $\mathrm{C} 42$ & $0.048(4)$ & $0.048(5)$ & $0.024(3)$ & $0.004(3)$ & $0.019(3)$ & $0.001(3)$ \\
\hline $\mathrm{C} 43$ & $0.036(3)$ & $0.039(4)$ & $0.022(3)$ & $0.004(2)$ & $0.019(2)$ & $0.000(3)$ \\
\hline $\mathrm{C} 44$ & $0.042(3)$ & $0.028(4)$ & $0.022(3)$ & $0.007(2)$ & $0.020(2)$ & $0.003(3)$ \\
\hline $\mathrm{C} 45$ & $0.033(3)$ & $0.030(4)$ & $0.019(3)$ & $-0.002(2)$ & $0.014(2)$ & $-0.001(2)$ \\
\hline $\mathrm{C} 46$ & $0.048(4)$ & $0.036(4)$ & $0.030(3)$ & $-0.004(3)$ & $0.022(3)$ & $-0.013(3)$ \\
\hline $\mathrm{C} 47$ & $0.060(5)$ & $0.056(5)$ & $0.039(4)$ & $-0.008(3)$ & $0.024(3)$ & $-0.014(4)$ \\
\hline $\mathrm{C} 48$ & $0.049(4)$ & $0.042(5)$ & $0.031(3)$ & $-0.007(3)$ & $0.020(3)$ & $-0.004(3)$ \\
\hline C49 & $0.045(4)$ & $0.044(4)$ & $0.034(3)$ & $-0.005(3)$ & $0.025(3)$ & $-0.009(3)$ \\
\hline $\mathrm{C} 50$ & $0.031(3)$ & $0.032(4)$ & $0.016(3)$ & $-0.005(2)$ & $0.016(2)$ & $-0.010(2)$ \\
\hline C51 & $0.032(3)$ & $0.046(4)$ & $0.017(3)$ & $0.004(2)$ & $0.010(2)$ & $-0.005(3)$ \\
\hline C52 & $0.033(3)$ & $0.045(4)$ & $0.017(3)$ & $0.003(2)$ & $0.013(2)$ & $0.001(3)$ \\
\hline C53 & $0.035(3)$ & $0.053(5)$ & $0.025(3)$ & $-0.002(3)$ & $0.013(3)$ & $-0.006(3)$ \\
\hline C54 & $0.029(3)$ & $0.073(6)$ & $0.026(3)$ & $0.004(3)$ & $0.016(3)$ & $0.005(3)$ \\
\hline C55 & $0.028(3)$ & $0.051(5)$ & $0.037(3)$ & $0.001(3)$ & $0.019(3)$ & $-0.001(3)$ \\
\hline C56 & $0.069(6)$ & $0.079(7)$ & $0.062(5)$ & $0.010(4)$ & $0.036(4)$ & $0.021(5)$ \\
\hline C57 & $0.088(7)$ & $0.078(8)$ & $0.069(6)$ & $-0.002(5)$ & $0.044(5)$ & $-0.008(5)$ \\
\hline C58 & $0.037(4)$ & $0.065(6)$ & $0.062(5)$ & $-0.003(4)$ & $0.030(3)$ & $-0.002(4)$ \\
\hline C59 & $0.052(5)$ & $0.090(8)$ & $0.088(6)$ & $0.029(6)$ & $0.034(5)$ & $0.011(5)$ \\
\hline
\end{tabular}




\begin{tabular}{|c|c|c|c|c|c|c|}
\hline & $\mathbf{U}_{11}$ & $\mathbf{U}_{22}$ & $\mathbf{U}_{33}$ & $\mathbf{U}_{23}$ & $\mathbf{U}_{13}$ & $\mathbf{U}_{12}$ \\
\hline C60 & $0.042(4)$ & $0.071(7)$ & $0.070(5)$ & $0.016(4)$ & $0.023(4)$ & $-0.007(4)$ \\
\hline C61 & $0.032(3)$ & $0.037(4)$ & $0.017(3)$ & $0.001(2)$ & $0.009(2)$ & $0.004(3)$ \\
\hline C62 & $0.043(4)$ & $0.047(5)$ & $0.024(3)$ & $0.003(3)$ & $0.013(3)$ & $-0.008(3)$ \\
\hline C63 & $0.055(4)$ & $0.033(4)$ & $0.025(3)$ & $-0.006(3)$ & $0.018(3)$ & $-0.005(3)$ \\
\hline C64 & $0.047(4)$ & $0.048(5)$ & $0.026(3)$ & $-0.003(3)$ & $0.012(3)$ & $0.004(3)$ \\
\hline C65 & $0.038(3)$ & $0.032(4)$ & $0.019(3)$ & $0.003(2)$ & $0.012(2)$ & $-0.001(3)$ \\
\hline C66 & $0.040(3)$ & $0.034(4)$ & $0.019(3)$ & $0.002(2)$ & $0.014(2)$ & $0.001(3)$ \\
\hline C67 & $0.065(6)$ & $0.077(8)$ & $0.102(7)$ & $-0.011(6)$ & $0.052(5)$ & $-0.013(5)$ \\
\hline C68 & $0.090(8)$ & $0.215(19)$ & $0.149(12)$ & $-0.068(12)$ & $0.083(8)$ & $-0.041(10)$ \\
\hline C69 & $0.054(5)$ & $0.080(8)$ & $0.083(6)$ & $-0.004(5)$ & $0.023(4)$ & $-0.004(5)$ \\
\hline $\mathrm{C} 70$ & $0.119(11)$ & $0.157(17)$ & $0.190(16)$ & $0.021(14)$ & $0.043(10)$ & $0.069(11)$ \\
\hline C71 & $0.045(4)$ & $0.064(6)$ & $0.035(4)$ & $-0.005(3)$ & $0.013(3)$ & $0.003(4)$ \\
\hline $\mathrm{C} 72$ & $0.061(5)$ & $0.082(7)$ & $0.068(5)$ & $-0.005(5)$ & $0.035(4)$ & $-0.004(5)$ \\
\hline $\mathrm{C} 73$ & $0.055(5)$ & $0.057(6)$ & $0.055(4)$ & $-0.007(4)$ & $0.020(4)$ & $-0.010(4)$ \\
\hline $\mathrm{C} 74$ & $0.053(5)$ & $0.082(7)$ & $0.061(5)$ & $-0.012(5)$ & $0.011(4)$ & $-0.003(4)$ \\
\hline C75 & $0.049(4)$ & $0.035(4)$ & $0.024(3)$ & $0.008(3)$ & $0.014(3)$ & $0.003(3)$ \\
\hline C76 & $0.075(5)$ & $0.055(5)$ & $0.020(3)$ & $0.010(3)$ & $0.016(3)$ & $-0.002(4)$ \\
\hline C77 & $0.053(4)$ & $0.059(6)$ & $0.039(4)$ & $0.001(3)$ & $0.019(3)$ & $-0.003(4)$ \\
\hline C78 & $0.061(5)$ & $0.057(5)$ & $0.037(4)$ & $0.014(3)$ & $0.028(3)$ & $0.013(4)$ \\
\hline C79 & $0.034(4)$ & $0.075(6)$ & $0.022(3)$ & $0.019(3)$ & $0.006(3)$ & $0.010(3)$ \\
\hline $\mathrm{C} 80$ & $0.054(5)$ & $0.128(10)$ & $0.051(5)$ & $0.025(5)$ & $0.012(4)$ & $0.005(5)$ \\
\hline $\mathrm{C} 81$ & $0.065(6)$ & $0.147(13)$ & $0.063(6)$ & $-0.001(6)$ & $0.013(5)$ & $-0.014(7)$ \\
\hline $\mathrm{C} 82$ & $0.041(4)$ & $0.078(7)$ & $0.041(4)$ & $0.000(4)$ & $-0.009(3)$ & $0.011(4)$ \\
\hline $\mathrm{C} 83$ & $0.078(7)$ & $0.100(9)$ & $0.062(6)$ & $0.022(5)$ & $0.018(5)$ & $0.015(6)$ \\
\hline $\mathrm{C} 84$ & $0.077(7)$ & $0.095(9)$ & $0.052(5)$ & $0.001(5)$ & $0.017(4)$ & $0.007(5)$ \\
\hline $\mathrm{C} 85$ & $0.052(5)$ & $0.080(8)$ & $0.063(5)$ & $-0.017(5)$ & $-0.008(4)$ & $0.014(5)$ \\
\hline $\mathrm{C} 86$ & $0.096(9)$ & $0.105(12)$ & $0.147(11)$ & $-0.037(9)$ & $0.030(8)$ & $-0.023(8)$ \\
\hline $\mathrm{C} 87$ & $0.052(5)$ & $0.077(7)$ & $0.059(5)$ & $-0.002(4)$ & $0.012(4)$ & $0.003(4)$ \\
\hline $\mathrm{C} 88$ & $0.087(8)$ & $0.213(18)$ & $0.083(8)$ & $-0.042(9)$ & $-0.002(6)$ & $0.060(9)$ \\
\hline C89 & $0.034(3)$ & $0.056(5)$ & $0.023(3)$ & $-0.005(3)$ & $0.019(3)$ & $-0.005(3)$ \\
\hline $\mathrm{C} 90$ & $0.036(3)$ & $0.037(4)$ & $0.021(3)$ & $-0.003(2)$ & $0.016(2)$ & $-0.009(3)$ \\
\hline C91 & $0.037(3)$ & $0.035(4)$ & $0.023(3)$ & $0.000(2)$ & $0.021(2)$ & $-0.001(3)$ \\
\hline
\end{tabular}




$\begin{array}{lllllll} & \mathbf{U}_{11} & \mathbf{U}_{22} & \mathbf{U}_{33} & \mathbf{U}_{23} & \mathbf{U}_{13} & \mathbf{U}_{12} \\ \text { C92 } & 0.045(4) & 0.047(5) & 0.034(3) & 0.000(3) & 0.027(3) & -0.001(3) \\ \text { C93 } & 0.053(4) & 0.045(5) & 0.035(3) & -0.004(3) & 0.021(3) & -0.001(3) \\ \text { C94 } & 0.048(4) & 0.038(4) & 0.028(3) & -0.003(3) & 0.022(3) & 0.005(3) \\ \text { C95 } & 0.047(4) & 0.045(4) & 0.025(3) & 0.002(3) & 0.020(3) & -0.005(3) \\ \text { C96 } & 0.054(5) & 0.079(7) & 0.034(4) & 0.008(4) & 0.017(3) & -0.007(4) \\ \text { C97 } & 0.065(5) & 0.051(5) & 0.033(3) & 0.006(3) & 0.017(3) & -0.008(4) \\ \text { C98 } & 0.079(6) & 0.074(6) & 0.038(4) & 0.002(4) & 0.040(4) & 0.011(4) \\ \text { C99 } & 0.042(4) & 0.052(5) & 0.045(4) & -0.004(3) & 0.014(3) & 0.006(3) \\ \text { C100 } & 0.060(6) & 0.116(10) & 0.099(7) & -0.008(7) & 0.040(5) & -0.011(6) \\ \text { C101 } & 0.052(4) & 0.044(5) & 0.058(4) & -0.010(4) & 0.023(3) & -0.001(3) \\ \text { C102 } & 0.054(5) & 0.098(8) & 0.045(4) & -0.007(4) & 0.012(4) & 0.014(5)\end{array}$

Table S16. Hydrogen atomic coordinates and isotropic atomic displacement parameters $\left(\AA^{2}\right)$ for FR-2.

$\begin{array}{lllll} & \mathbf{x} / \mathbf{a} & \mathbf{y} / \mathbf{b} & \mathbf{z} / \mathbf{c} & \mathbf{U}(\mathbf{e q}) \\ \mathrm{H} 3 & 0.1596 & 0.7429 & 0.1074 & 0.05 \\ \mathrm{H} 4 & 0.1619 & 0.6778 & 0.1692 & 0.046 \\ \mathrm{H} 9 & 0.1478 & 0.4214 & 0.1812 & 0.047 \\ \mathrm{H} 10 & 0.1850 & 0.2959 & 0.1921 & 0.056 \\ \mathrm{H} 11 & 0.2158 & 0.2736 & 0.2553 & 0.047 \\ \mathrm{H} 12 & 0.2112 & 0.3866 & 0.3072 & 0.048 \\ \mathrm{H} 16 & 0.1610 & 0.7072 & 0.3595 & 0.038 \\ \mathrm{H} 17 & 0.1275 & 0.8553 & 0.3489 & 0.047 \\ \mathrm{H} 18 & 0.0994 & 0.9023 & 0.2839 & 0.046 \\ \mathrm{H} 19 & 0.1024 & 0.7761 & 0.2314 & 0.043 \\ \mathrm{H} 22 & 0.0799 & 0.5165 & 0.2278 & 0.056 \\ \mathrm{H} 23 & 0.0349 & 0.5137 & 0.1986 & 0.057 \\ \mathrm{H} 25 & 0.0400 & 0.6524 & 0.0952 & 0.052 \\ \mathrm{H} 28 & 0.0793 & 0.7176 & 0.0668 & 0.033 \\ \mathrm{H} 29 & 0.0792 & 0.7921 & 0.0127 & 0.039 \\ \mathrm{H} 32 & 0.0395 & 0.8567 & -0.0554 & 0.042\end{array}$




\begin{tabular}{|c|c|c|c|c|}
\hline & $\mathbf{x} / \mathbf{a}$ & $y / b$ & $\mathbf{z} / \mathbf{c}$ & $\mathbf{U}(\mathbf{e q})$ \\
\hline H34 & 0.0342 & 0.9884 & -0.1642 & 0.059 \\
\hline H35 & 0.0798 & 0.9778 & -0.1490 & 0.047 \\
\hline H39 & 0.1026 & 0.7223 & -0.1291 & 0.048 \\
\hline $\mathrm{H} 40$ & 0.0991 & 0.5976 & -0.1840 & 0.048 \\
\hline H41 & 0.1273 & 0.6436 & -0.2221 & 0.039 \\
\hline $\mathrm{H} 42$ & 0.1605 & 0.7893 & -0.1997 & 0.046 \\
\hline H46 & 0.2111 & 1.1115 & -0.0971 & 0.043 \\
\hline $\mathrm{H} 47$ & 0.2144 & 1.2381 & -0.0422 & 0.06 \\
\hline $\mathrm{H} 48$ & 0.1822 & 1.2165 & -0.0105 & 0.047 \\
\hline H49 & 0.1467 & 1.0806 & -0.0349 & 0.045 \\
\hline H53 & 0.1617 & 0.8373 & -0.0085 & 0.044 \\
\hline H54 & 0.1606 & 0.7712 & 0.0525 & 0.049 \\
\hline H56 & -0.0054 & 0.4148 & 0.1506 & 0.079 \\
\hline H57 & -0.0506 & 0.4171 & 0.1237 & 0.089 \\
\hline H59 & -0.0460 & 0.7719 & 0.0733 & 0.089 \\
\hline H60 & 0.0000 & 0.7465 & 0.0947 & 0.072 \\
\hline H62 & 0.2246 & 0.6334 & 0.3357 & 0.045 \\
\hline H64 & 0.2457 & 0.5169 & 0.4499 & 0.049 \\
\hline H66 & 0.1719 & 0.4546 & 0.3762 & 0.036 \\
\hline H68A & -0.0821 & 0.6729 & 0.1380 & 0.211 \\
\hline H68B & -0.0952 & 0.5347 & 0.1266 & 0.211 \\
\hline $\mathrm{H} 68 \mathrm{C}$ & -0.1119 & 0.6613 & 0.1103 & 0.211 \\
\hline H69A & -0.1146 & 0.5082 & 0.0409 & 0.108 \\
\hline H69B & -0.0950 & 0.4252 & 0.0754 & 0.108 \\
\hline H69C & -0.0861 & 0.4721 & 0.0399 & 0.108 \\
\hline $\mathrm{H} 70 \mathrm{~A}$ & -0.0836 & 0.8131 & 0.0656 & 0.236 \\
\hline H70B & -0.1123 & 0.7538 & 0.0469 & 0.236 \\
\hline $\mathrm{H} 70 \mathrm{C}$ & -0.0903 & 0.7228 & 0.0280 & 0.236 \\
\hline $\mathrm{H} 72 \mathrm{~A}$ & 0.2670 & 0.6150 & 0.3437 & 0.1 \\
\hline $\mathrm{H} 72 \mathrm{~B}$ & 0.2924 & 0.6934 & 0.3687 & 0.1 \\
\hline $\mathrm{H} 72 \mathrm{C}$ & 0.2645 & 0.7629 & 0.3536 & 0.1 \\
\hline H73A & 0.2560 & 0.8231 & 0.4152 & 0.083 \\
\hline
\end{tabular}




\begin{tabular}{|c|c|c|c|c|}
\hline & $\mathbf{x} / \mathbf{a}$ & $\mathbf{y} / \mathbf{b}$ & $\mathbf{z} / \mathbf{c}$ & $\mathrm{U}(\mathrm{eq})$ \\
\hline H73B & 0.2872 & 0.8122 & 0.4285 & 0.083 \\
\hline $\mathrm{H} 73 \mathrm{C}$ & 0.2717 & 0.7465 & 0.4541 & 0.083 \\
\hline $\mathrm{H} 74 \mathrm{~A}$ & 0.2895 & 0.5331 & 0.4517 & 0.101 \\
\hline H74B & 0.3069 & 0.5767 & 0.4261 & 0.101 \\
\hline $\mathrm{H} 74 \mathrm{C}$ & 0.2863 & 0.4621 & 0.4115 & 0.101 \\
\hline $\mathrm{H} 76 \mathrm{~A}$ & 0.2150 & 0.4223 & 0.5144 & 0.075 \\
\hline H76B & 0.2124 & 0.5628 & 0.4959 & 0.075 \\
\hline $\mathrm{H} 76 \mathrm{C}$ & 0.2361 & 0.4719 & 0.4956 & 0.075 \\
\hline $\mathrm{H} 77 \mathrm{~A}$ & 0.2251 & 0.2641 & 0.4595 & 0.074 \\
\hline H77B & 0.1963 & 0.2388 & 0.4293 & 0.074 \\
\hline $\mathrm{H} 77 \mathrm{C}$ & 0.2013 & 0.2373 & 0.4752 & 0.074 \\
\hline $\mathrm{H} 78 \mathrm{~A}$ & 0.1603 & 0.4057 & 0.4230 & 0.073 \\
\hline H78B & 0.1680 & 0.5346 & 0.4481 & 0.073 \\
\hline $\mathrm{H} 78 \mathrm{C}$ & 0.1670 & 0.4012 & 0.4692 & 0.073 \\
\hline H80 & -0.0004 & 0.7540 & -0.0940 & 0.095 \\
\hline H81 & -0.0461 & 0.7330 & -0.1158 & 0.113 \\
\hline H83 & -0.0509 & 1.0745 & -0.1739 & 0.098 \\
\hline H84 & -0.0056 & 1.0819 & -0.1558 & 0.091 \\
\hline H86A & -0.0873 & 0.7783 & -0.1166 & 0.178 \\
\hline H86B & -0.0876 & 0.6906 & -0.1529 & 0.178 \\
\hline $\mathrm{H} 86 \mathrm{C}$ & -0.1134 & 0.7672 & -0.1537 & 0.178 \\
\hline H87A & -0.1156 & 0.9980 & -0.1652 & 0.097 \\
\hline H87B & -0.0895 & 1.0738 & -0.1632 & 0.097 \\
\hline $\mathrm{H} 87 \mathrm{C}$ & -0.0917 & 1.0060 & -0.1250 & 0.097 \\
\hline H88A & -0.0868 & 0.8019 & -0.2196 & 0.203 \\
\hline H88B & -0.0888 & 0.9549 & -0.2214 & 0.203 \\
\hline $\mathrm{H} 88 \mathrm{C}$ & -0.1138 & 0.8707 & -0.2228 & 0.203 \\
\hline H90 & 0.1715 & 1.0452 & -0.2046 & 0.036 \\
\hline H92 & 0.2457 & 0.9847 & -0.2044 & 0.046 \\
\hline H94 & 0.2242 & 0.8645 & -0.1122 & 0.043 \\
\hline H96A & 0.1600 & 1.0980 & -0.2671 & 0.082 \\
\hline H96B & 0.1683 & 0.9575 & -0.2762 & 0.082 \\
\hline
\end{tabular}




$\begin{array}{lcccc} & \mathbf{x} / \mathbf{a} & \mathbf{y} / \mathbf{b} & \mathbf{z} / \mathbf{c} & \mathbf{U}(\mathbf{e q}) \\ \text { H96C } & 0.1680 & 1.0737 & -0.3050 & 0.082 \\ \text { H97A } & 0.2029 & 1.2617 & -0.2742 & 0.074 \\ \text { H97B } & 0.2239 & 1.2360 & -0.2319 & 0.074 \\ \text { H97C } & 0.1939 & 1.2609 & -0.2366 & 0.074 \\ \text { H98A } & 0.2130 & 1.0473 & -0.3031 & 0.088 \\ \text { H98B } & 0.2146 & 0.9194 & -0.2781 & 0.088 \\ \text { H98C } & 0.2364 & 1.0283 & -0.2626 & 0.088 \\ \text { H10A } & 0.2894 & 0.9713 & -0.1632 & 0.132 \\ \text { H10B } & 0.3073 & 0.9242 & -0.1208 & 0.132 \\ \text { H10C } & 0.2872 & 1.0401 & -0.1252 & 0.132 \\ \text { H10D } & 0.2627 & 0.6602 & -0.1466 & 0.076 \\ \text { H10E } & 0.2897 & 0.7116 & -0.1500 & 0.076 \\ \text { H10F } & 0.2627 & 0.7393 & -0.1843 & 0.076 \\ \text { H10G } & 0.2918 & 0.8058 & -0.0771 & 0.101 \\ \text { H10H } & 0.2631 & 0.7449 & -0.0888 & 0.101 \\ \text { H10I } & 0.2675 & 0.8931 & -0.0771 & 0.101\end{array}$




\section{Appendix: ${ }^{1} \mathrm{H},{ }^{13} \mathrm{C}$ NMR and HR mass spectra of new compounds}

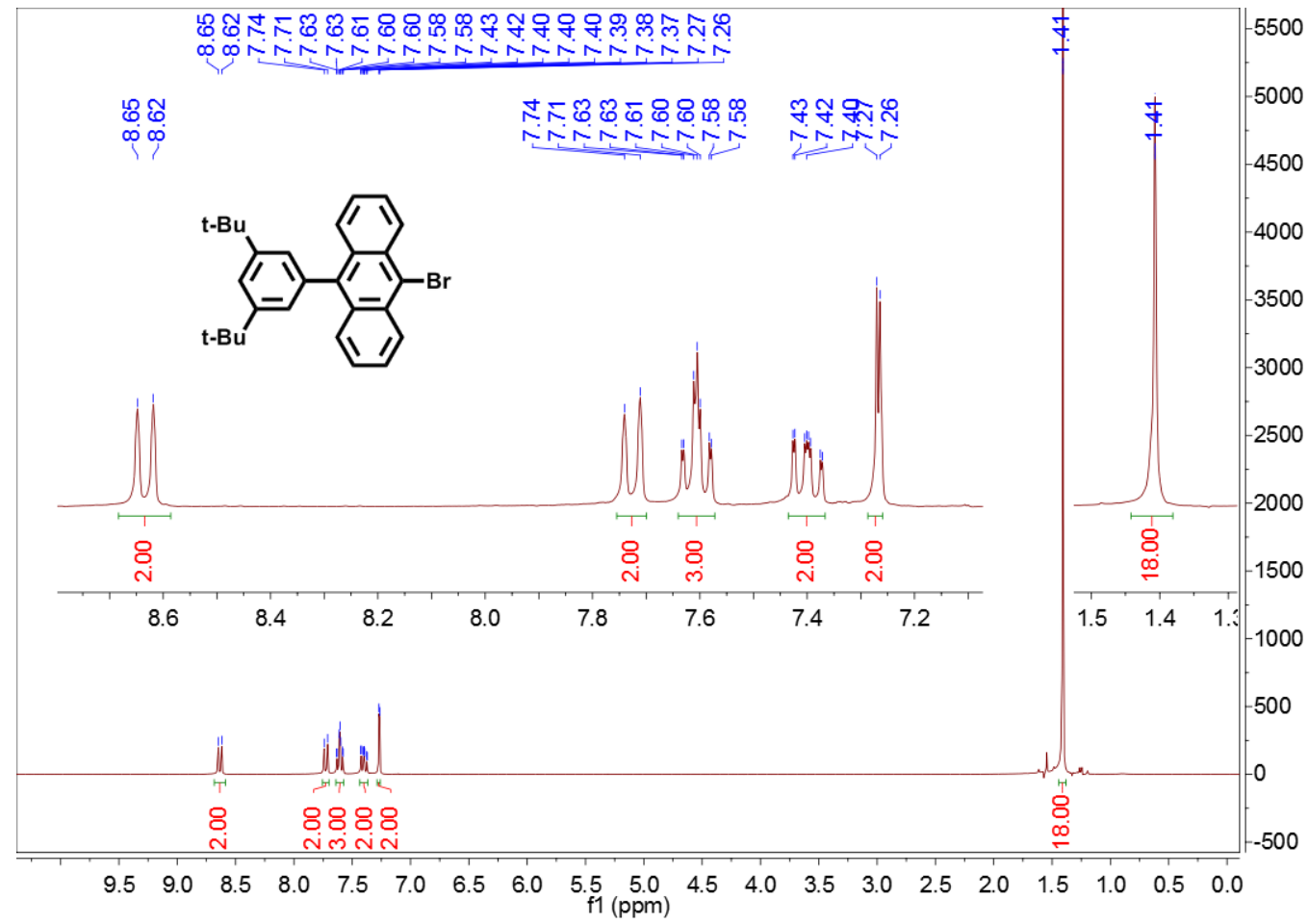

Figure S13. ${ }^{1} \mathrm{H}$ NMR spectrum $(300 \mathrm{MHz})$ of compound 2 in $\mathrm{CDCl}_{3}$ at $298 \mathrm{~K}$.

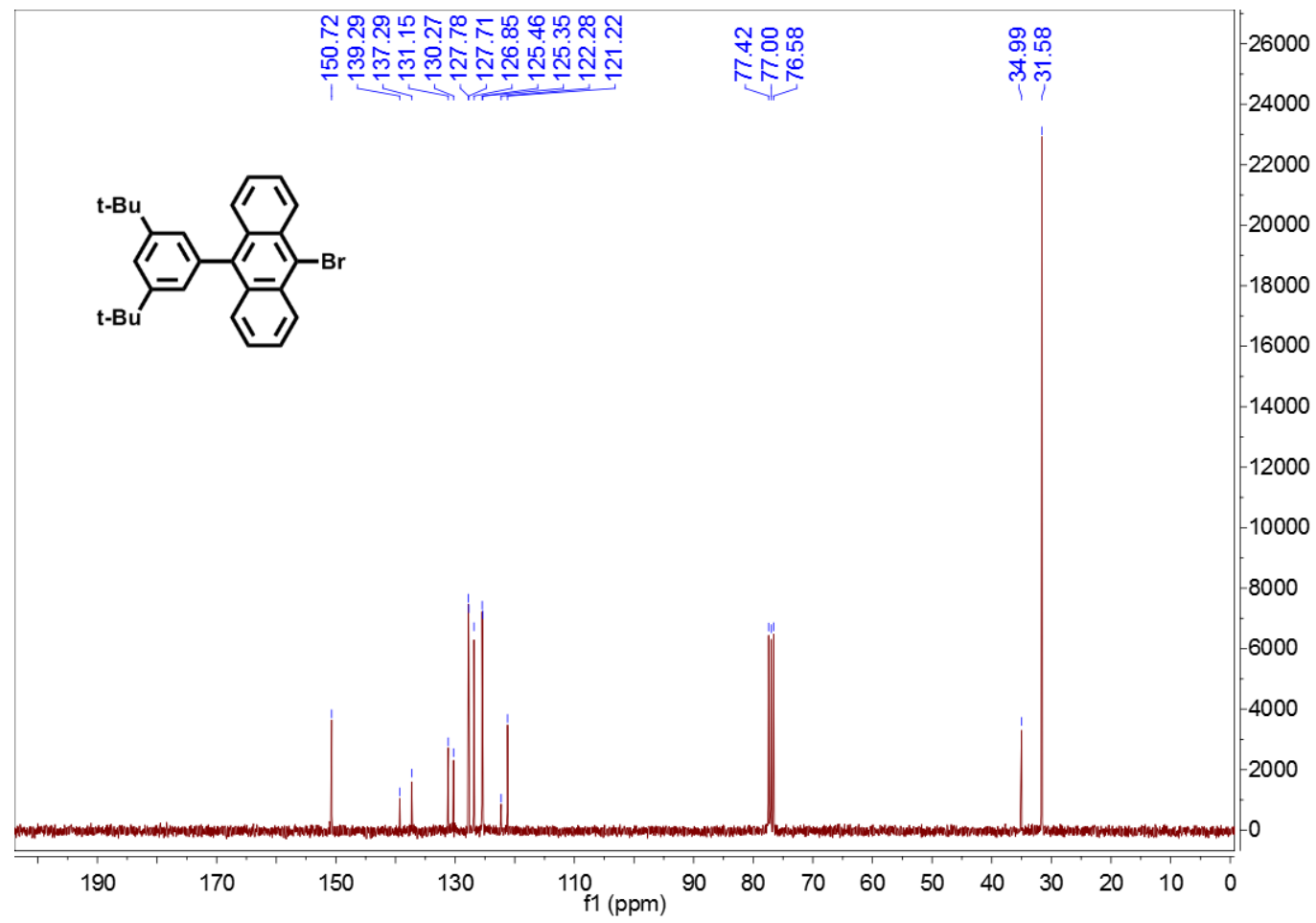

Figure S14. ${ }^{13} \mathrm{C}$ NMR spectrum $(75 \mathrm{MHz})$ of compound 2 in $\mathrm{CDCl}_{3}$ at $298 \mathrm{~K}$. 


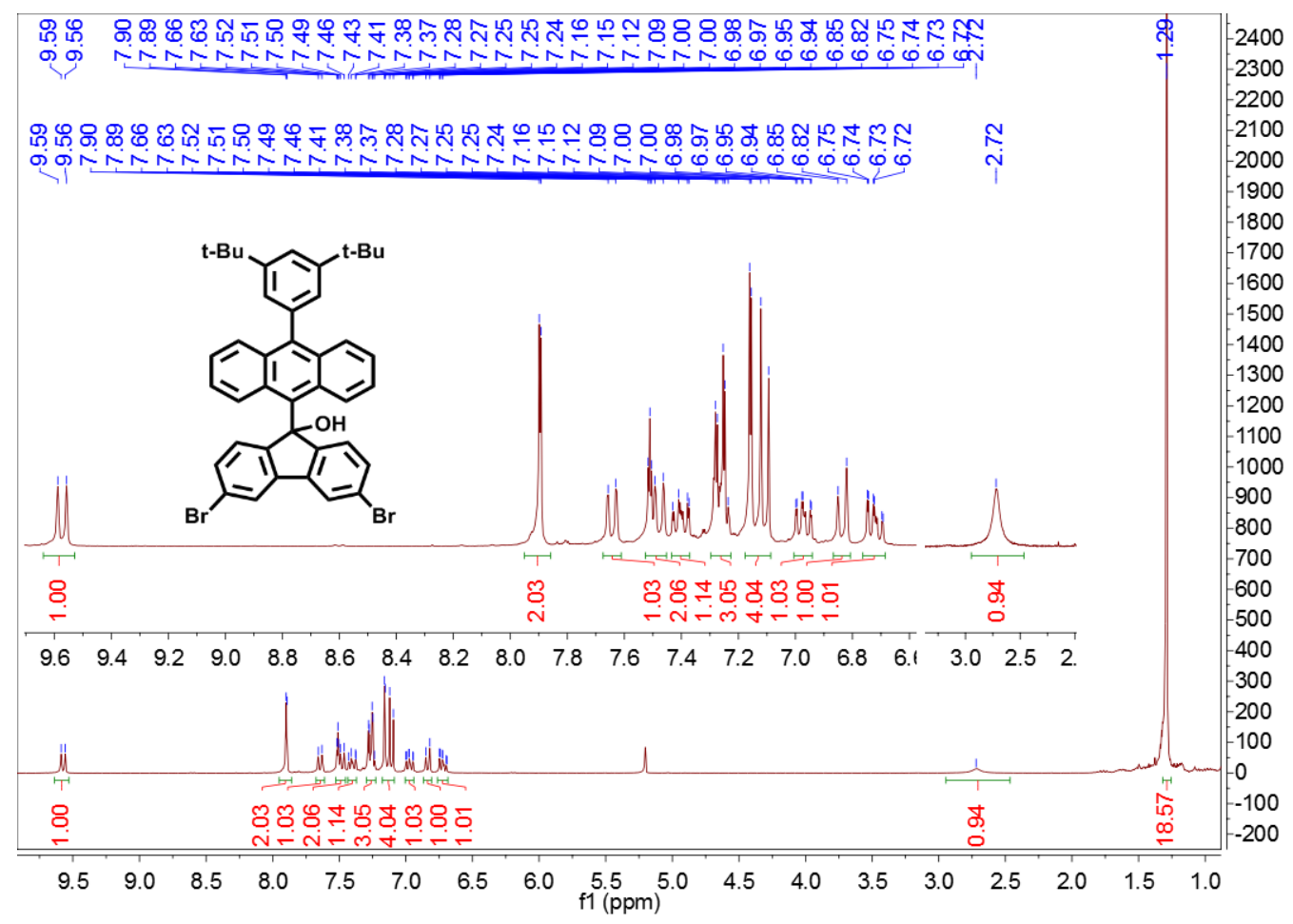

Figure S15. ${ }^{1} \mathrm{H}$ NMR spectrum (300 MHz) of compound 3 in $\mathrm{CD}_{2} \mathrm{Cl}_{2}$ at $298 \mathrm{~K}$.

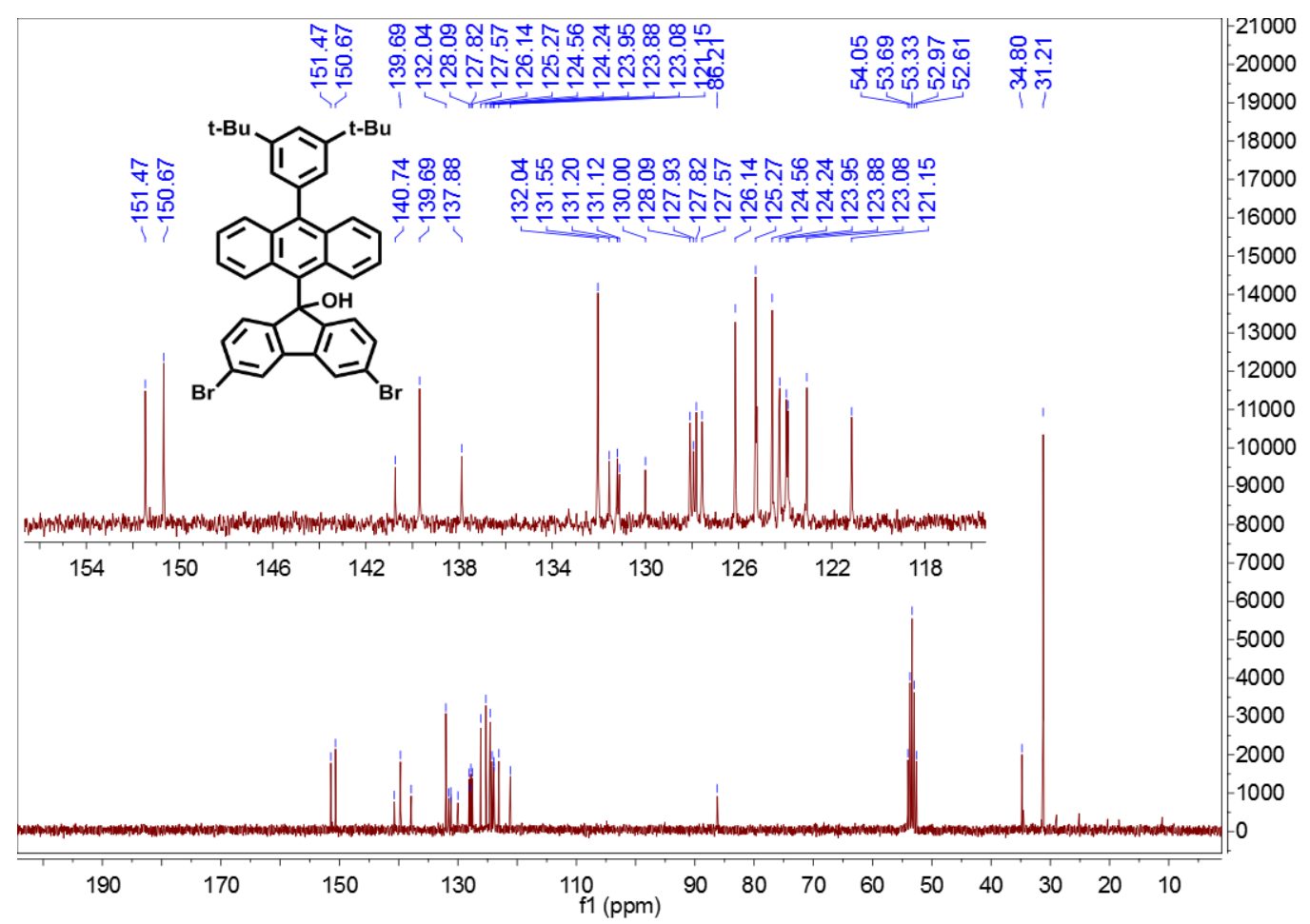

Figure S16. ${ }^{13} \mathrm{C}$ NMR spectrum $\left(75 \mathrm{MHz}\right.$ ) of compound 3 in $\mathrm{CD}_{2} \mathrm{Cl}_{2}$ at $298 \mathrm{~K}$. 


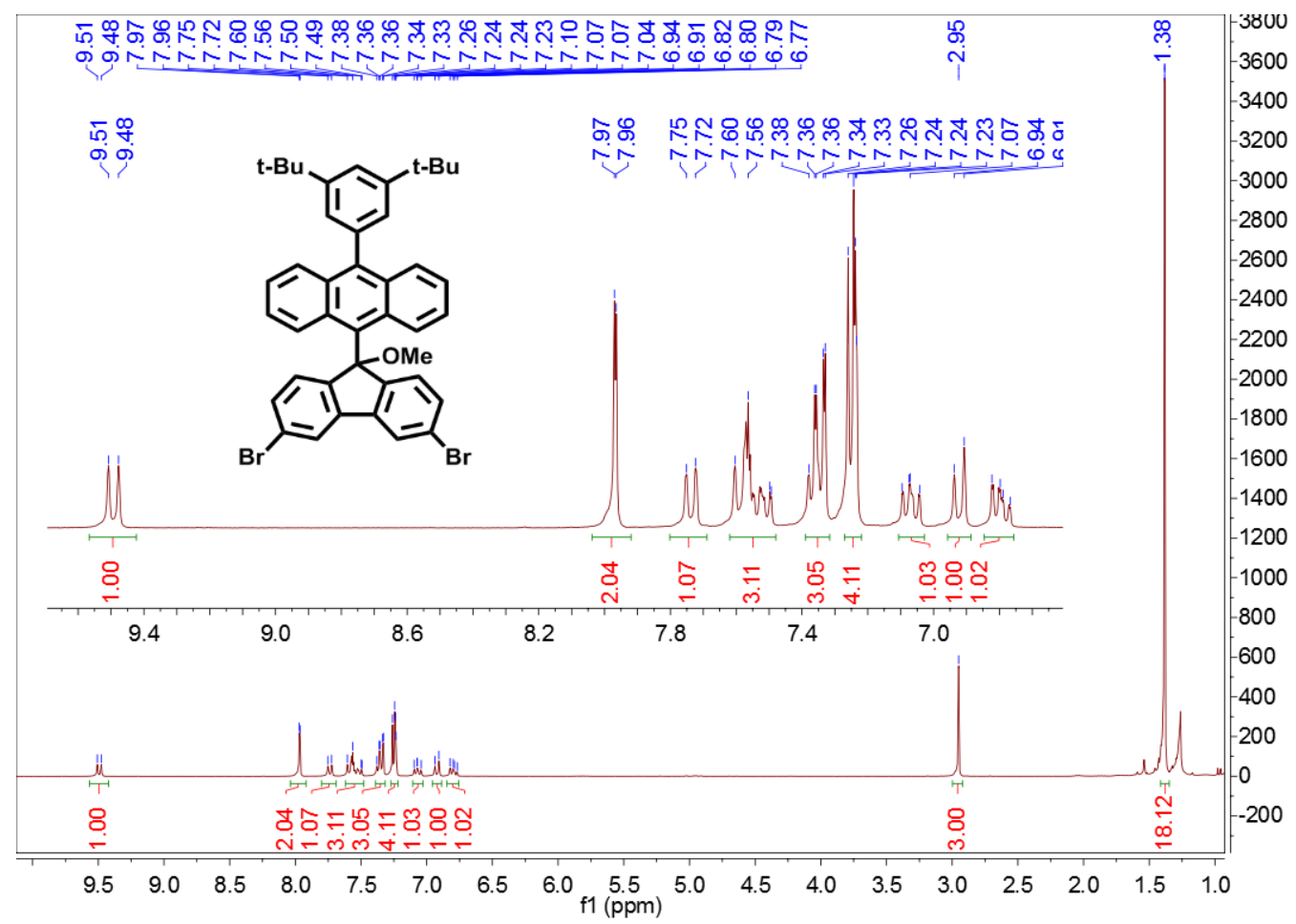

Figure S17. ${ }^{1} \mathrm{H}$ NMR spectrum (300 MHz) of compound 4 in $\mathrm{CDCl}_{3}$ at $298 \mathrm{~K}$.

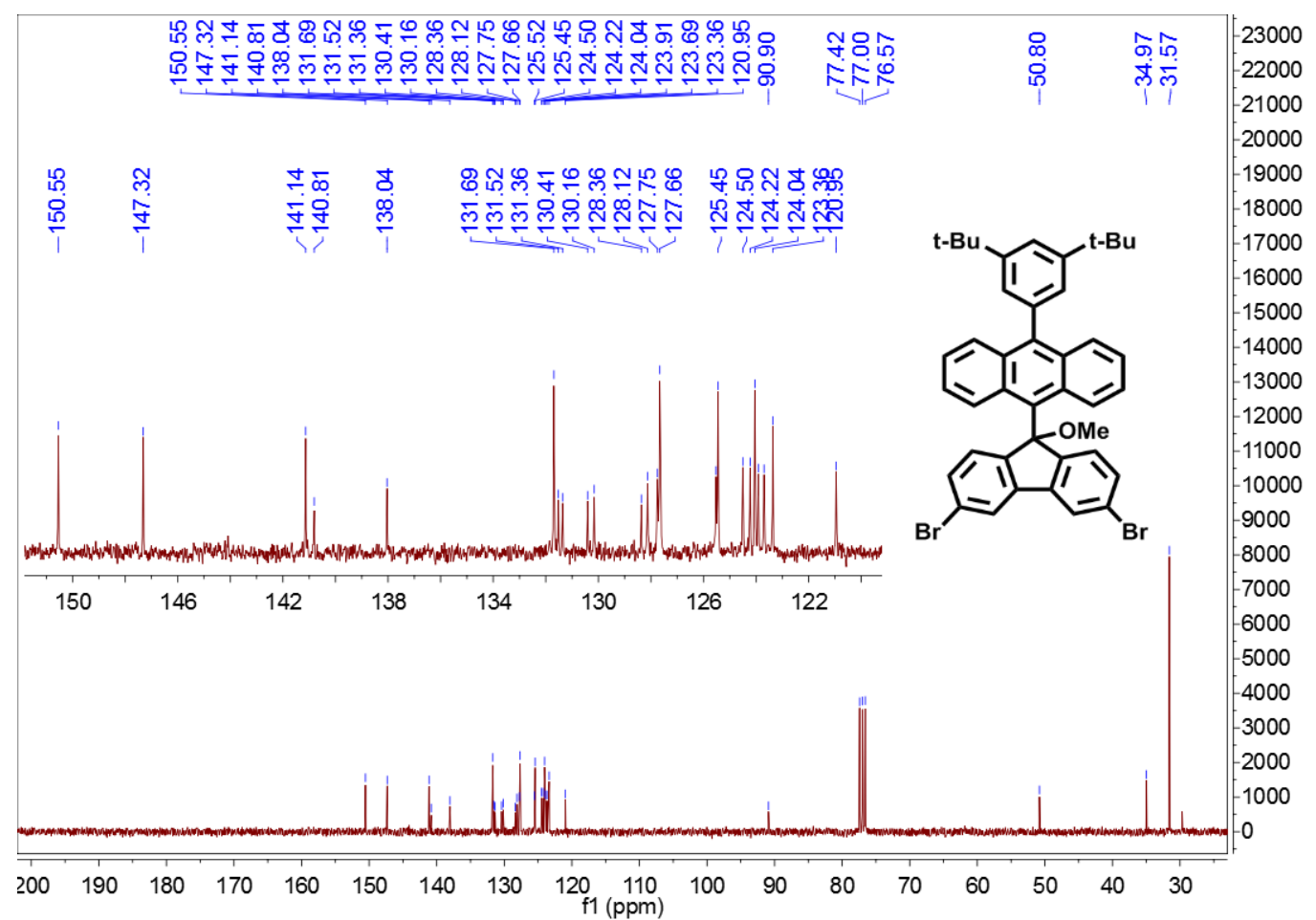

Figure S18. ${ }^{13} \mathrm{C}$ NMR spectrum $(75 \mathrm{MHz})$ of compound 4 in $\mathrm{CDCl}_{3}$ at $298 \mathrm{~K}$. 


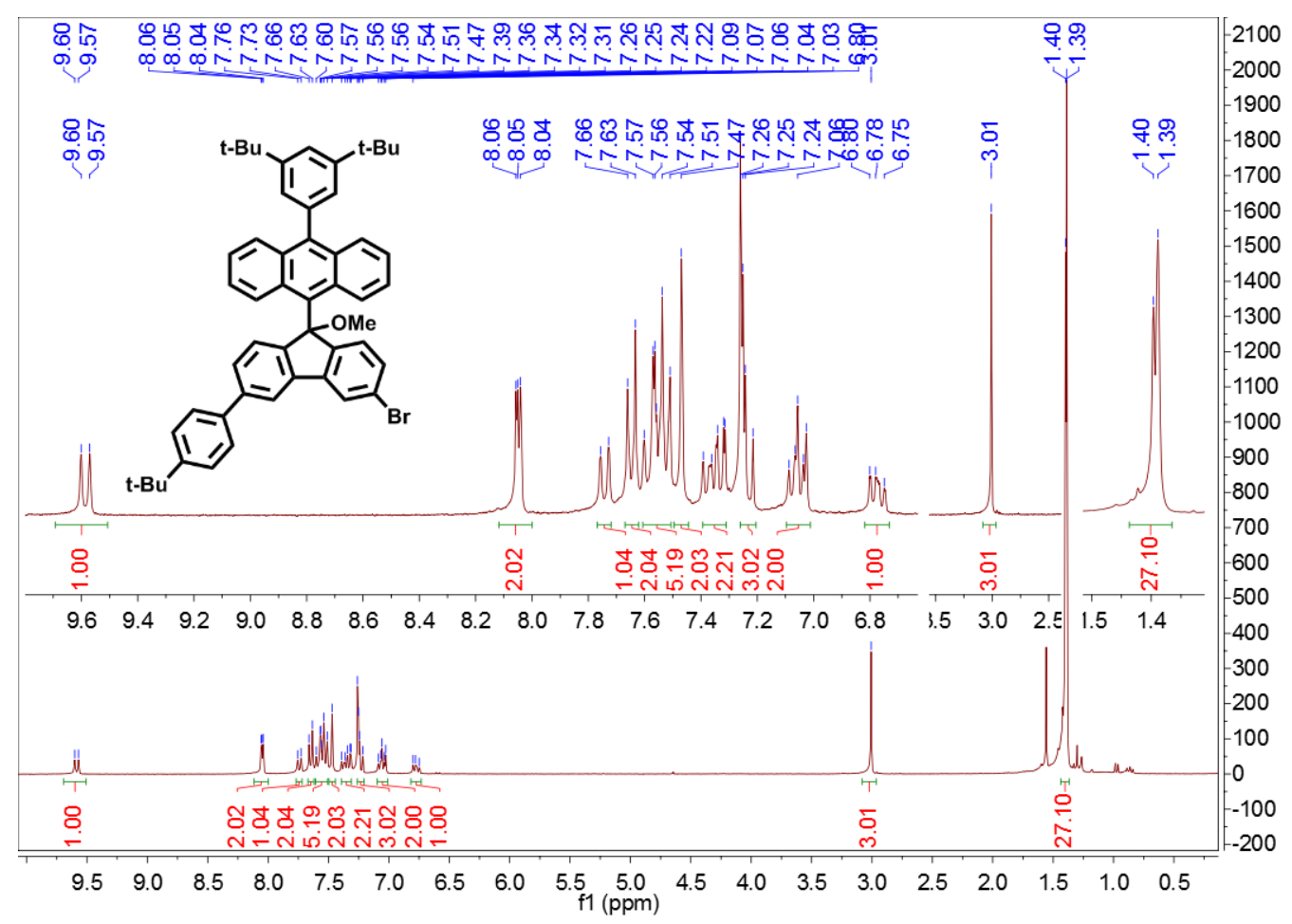

Figure S19. ${ }^{1} \mathrm{H}$ NMR spectrum $(300 \mathrm{MHz})$ of 5 in $\mathrm{CDCl}_{3}$ at $298 \mathrm{~K}$.

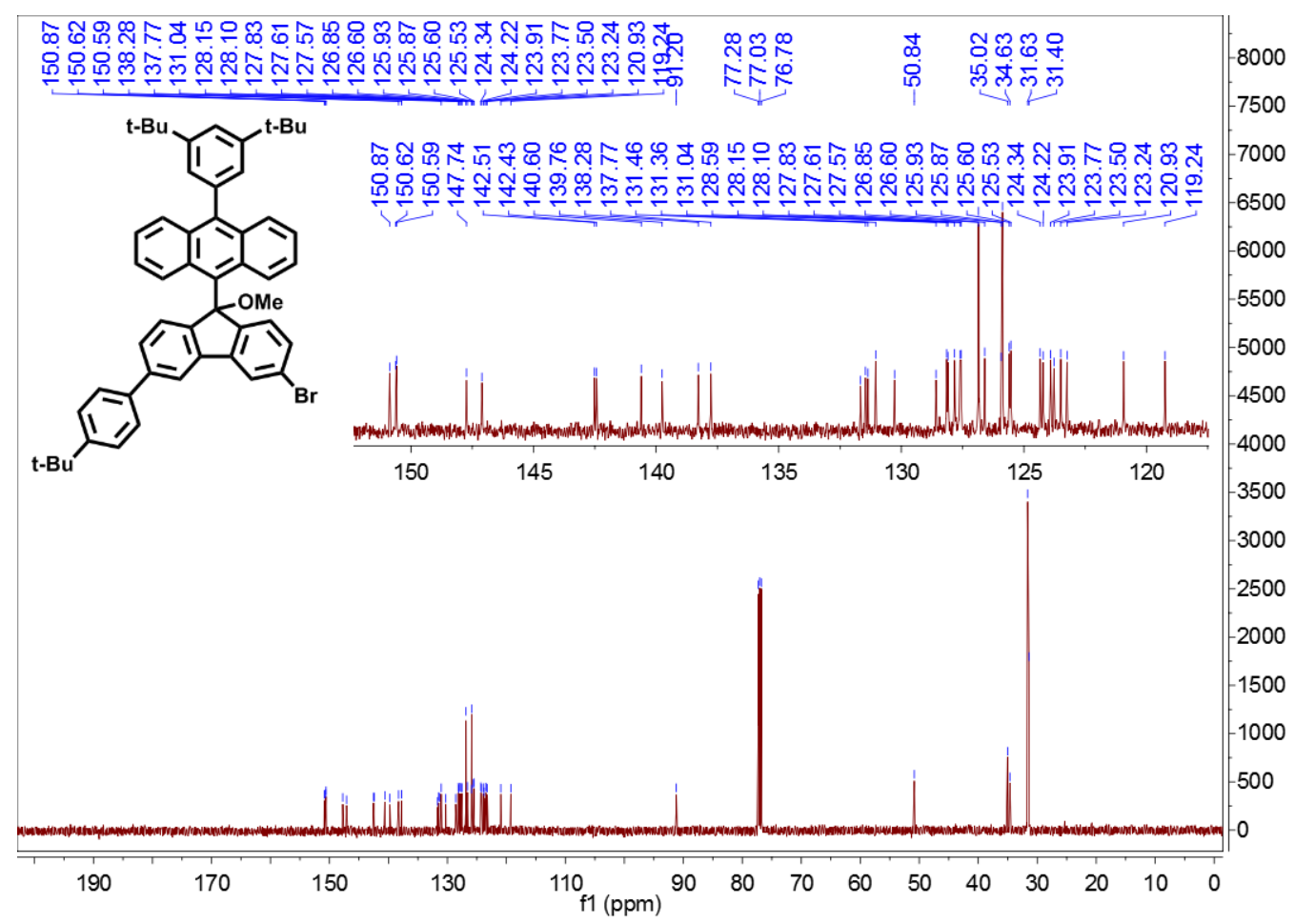

Figure S20. ${ }^{13} \mathrm{C}$ NMR spectrum $(75 \mathrm{MHz})$ of 5 in $\mathrm{CDCl}_{3}$ at $298 \mathrm{~K}$. 


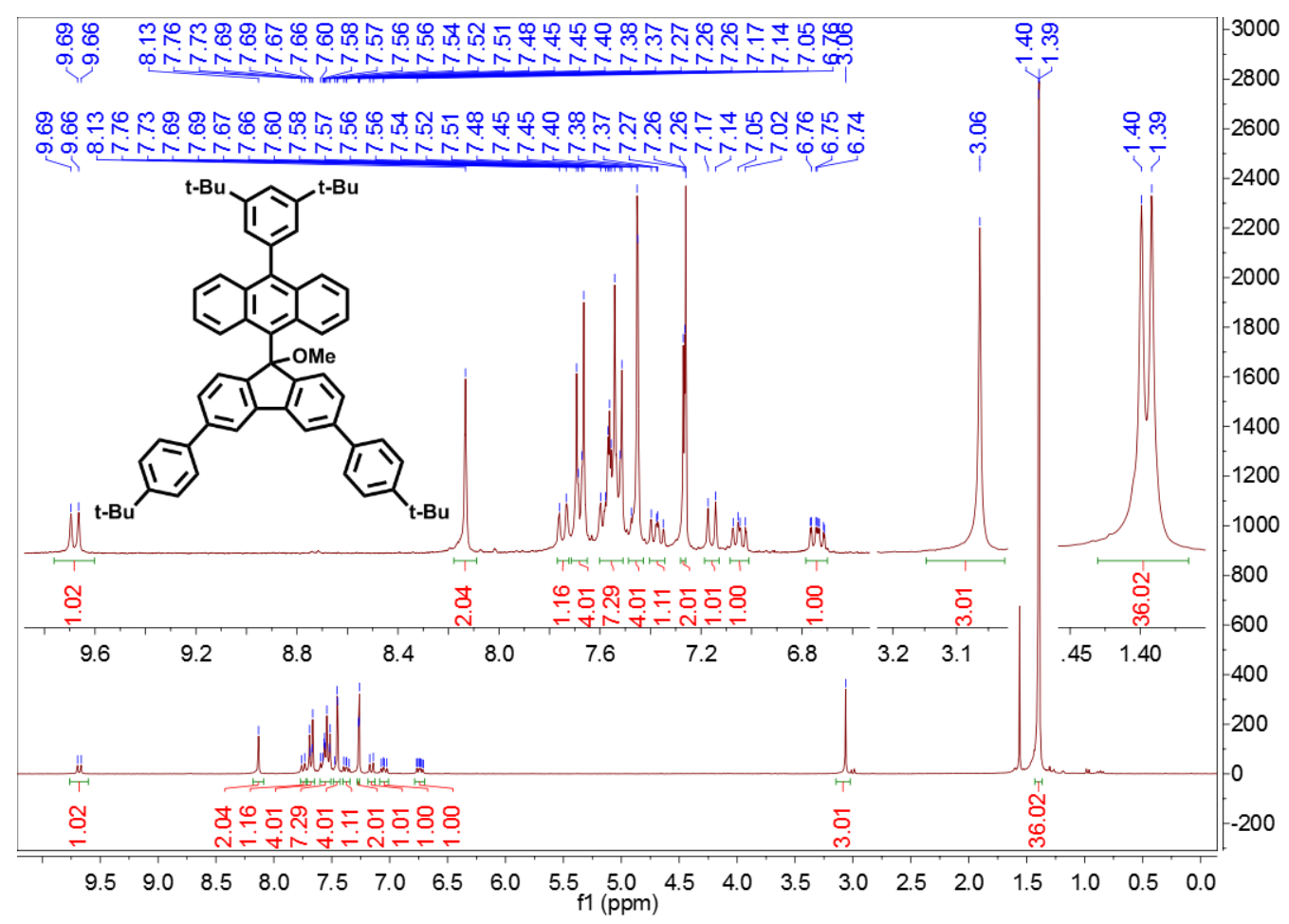

Figure S21. ${ }^{1} \mathrm{H}$ NMR spectrum $(300 \mathrm{MHz})$ of 6 in $\mathrm{CDCl}_{3}$ at $298 \mathrm{~K}$.

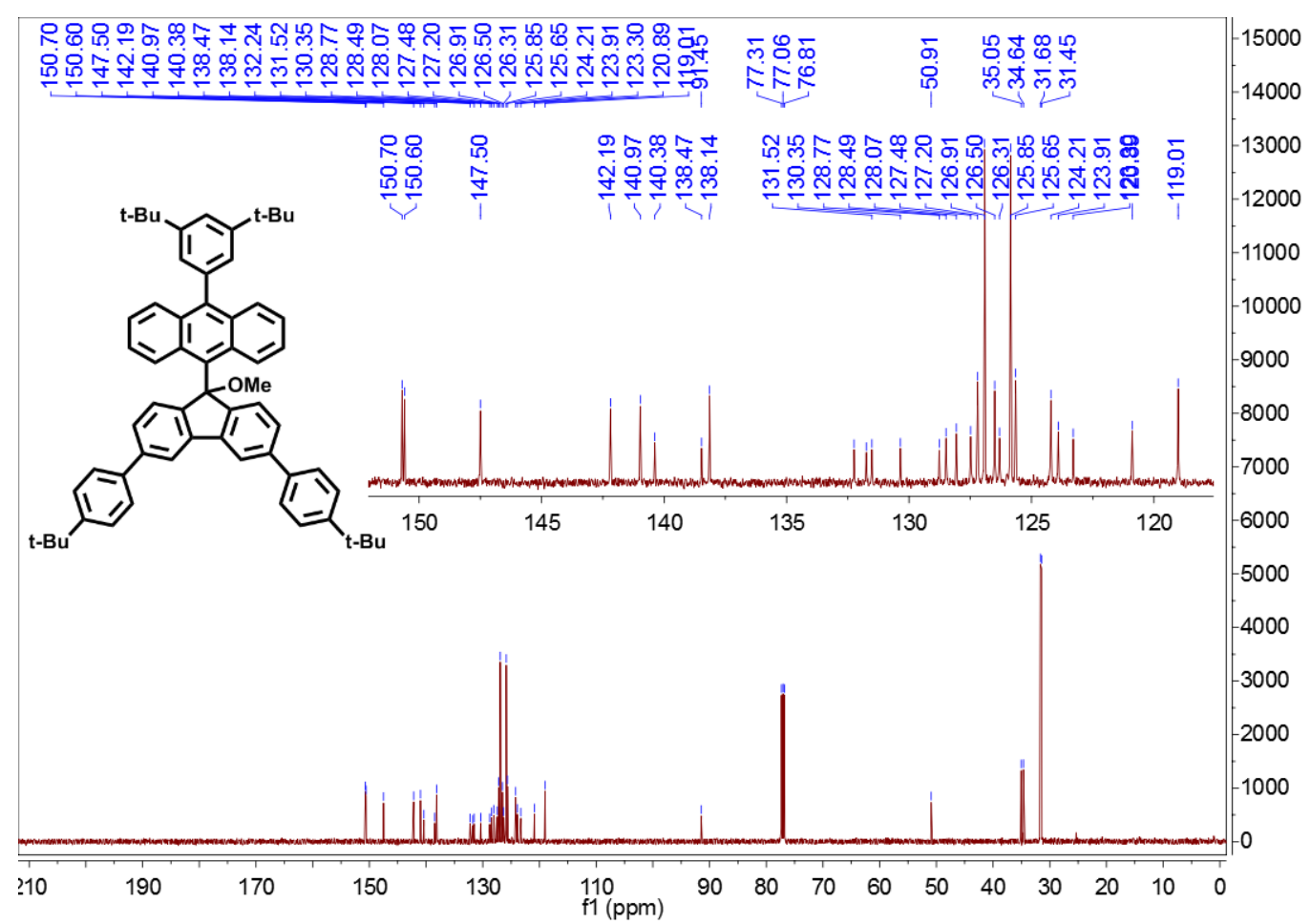

Figure S22. ${ }^{13} \mathrm{C}$ NMR spectrum $(75 \mathrm{MHz})$ of 6 in $\mathrm{CDCl}_{3}$ at $298 \mathrm{~K}$. 


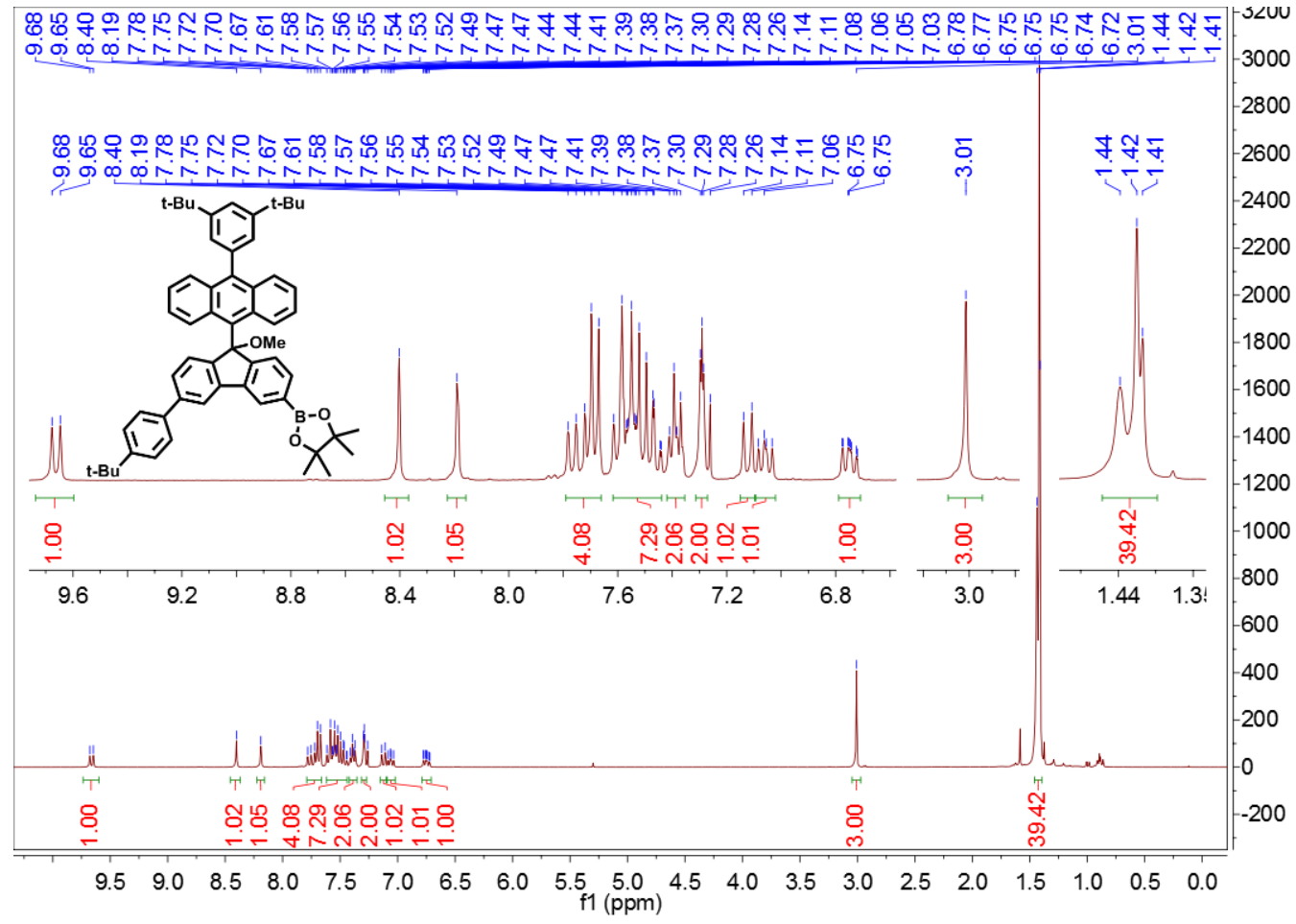

Figure S23. ${ }^{1} \mathrm{H}$ NMR spectrum $(300 \mathrm{MHz})$ of 7 in $\mathrm{CDCl}_{3}$ at $298 \mathrm{~K}$.

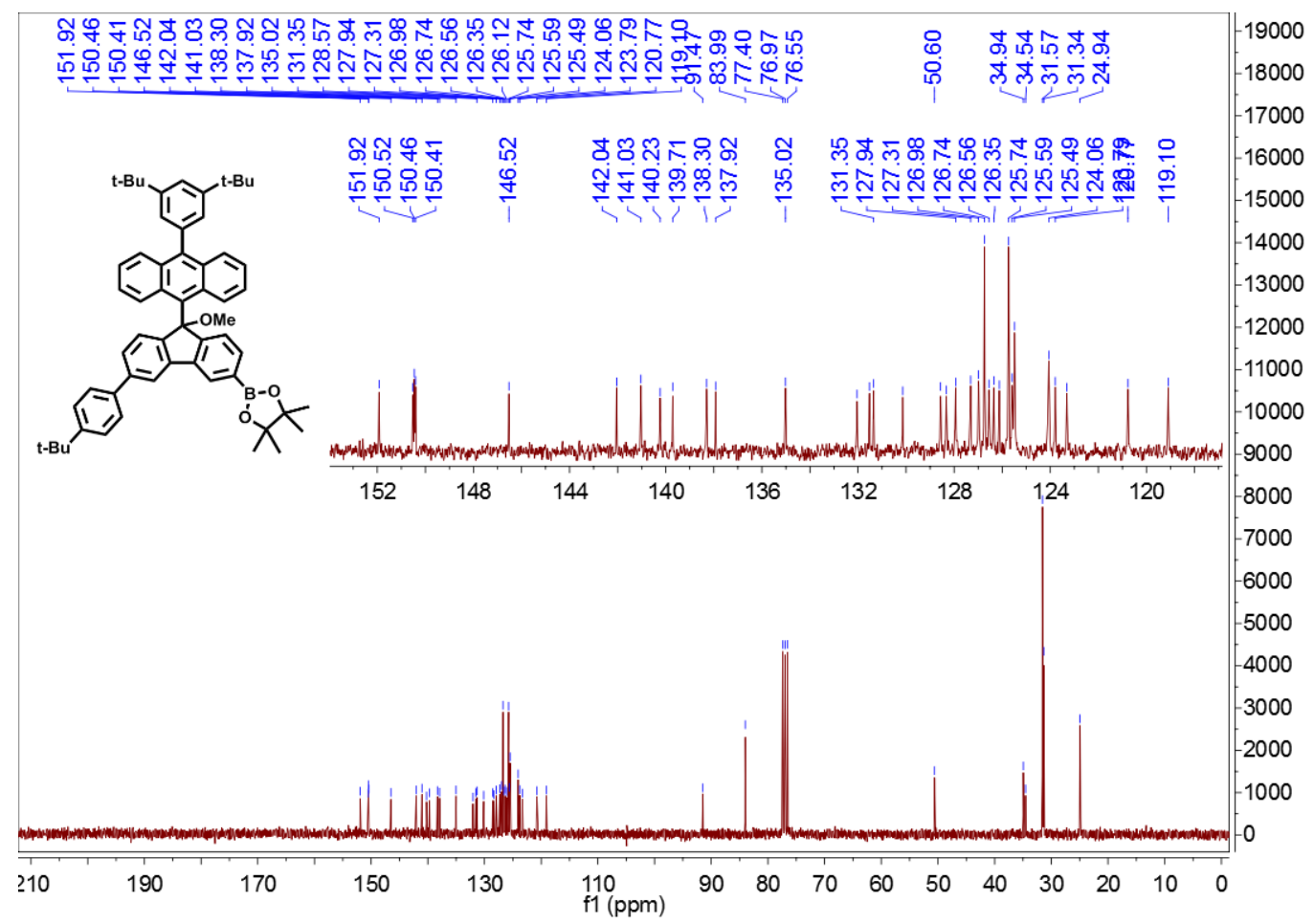

Figure S24. ${ }^{13} \mathrm{C}$ NMR spectrum $(75 \mathrm{MHz})$ of 7 in $\mathrm{CDCl}_{3}$ at $298 \mathrm{~K}$. 


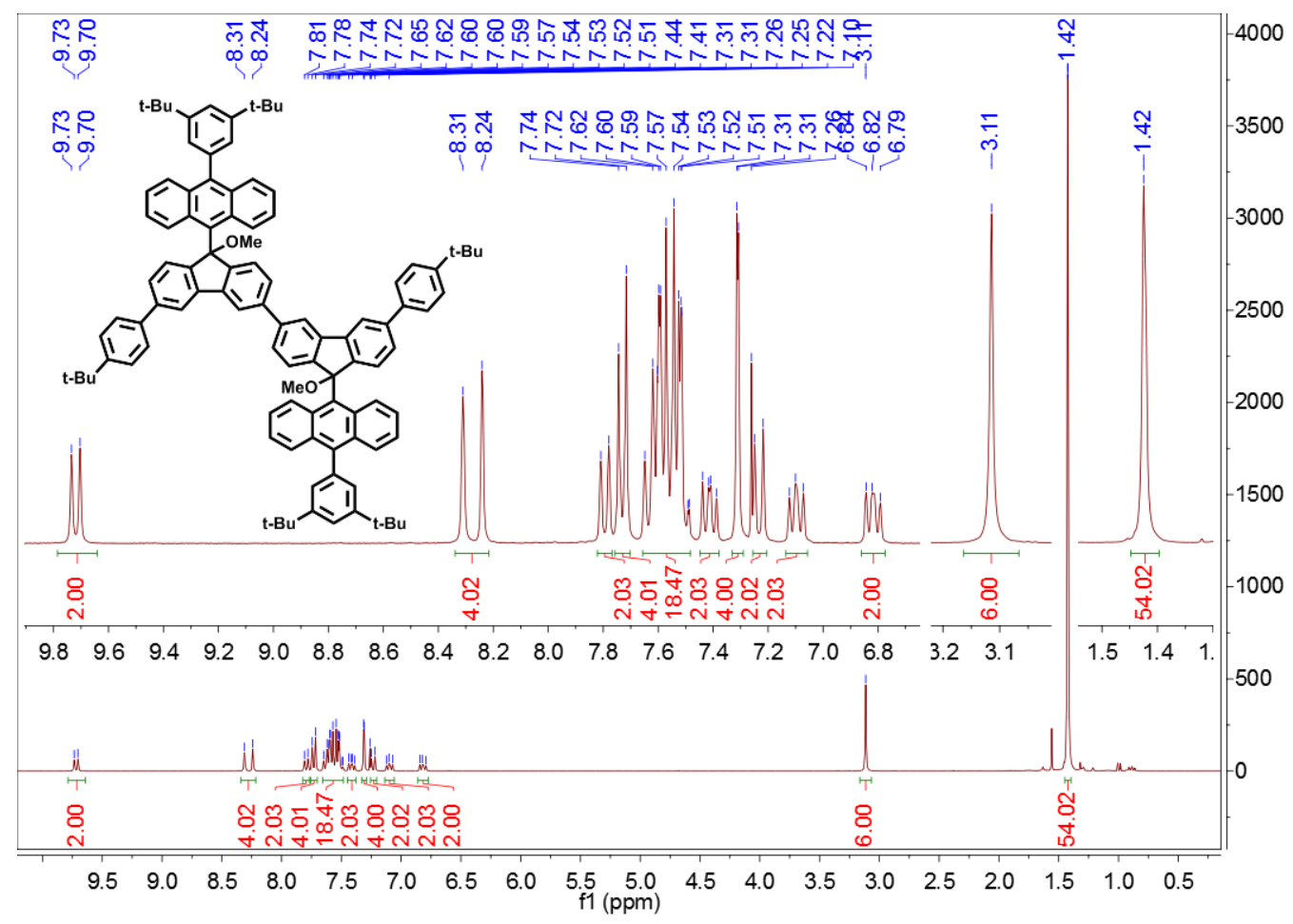

Figure S25. ${ }^{1} \mathrm{H}$ NMR spectrum $(300 \mathrm{MHz})$ of 8 in $\mathrm{CDCl}_{3}$ at $298 \mathrm{~K}$.

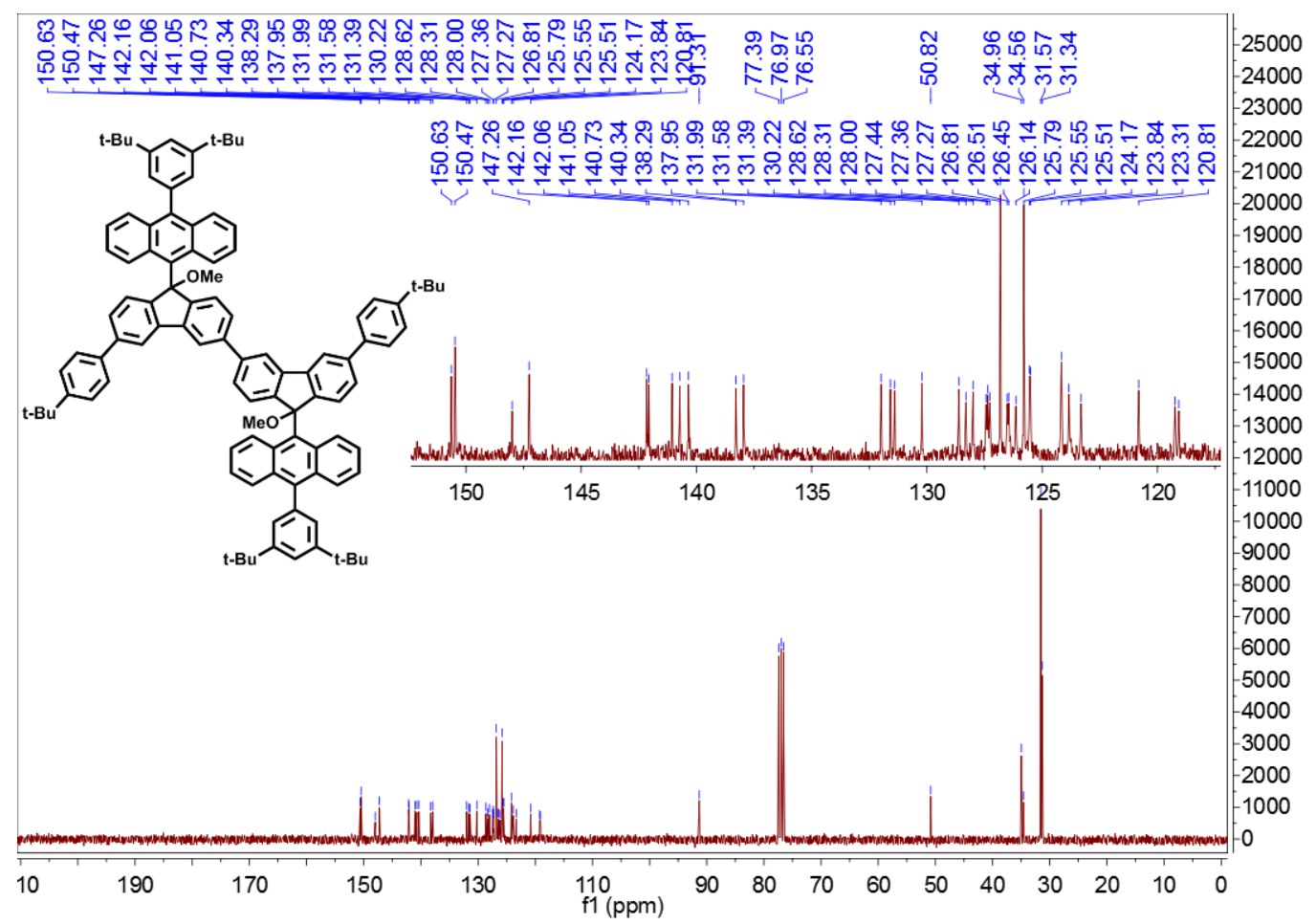

Figure S26. ${ }^{13} \mathrm{C}$ NMR spectrum $(75 \mathrm{MHz})$ of 8 in $\mathrm{CDCl}_{3}$ at $298 \mathrm{~K}$. 


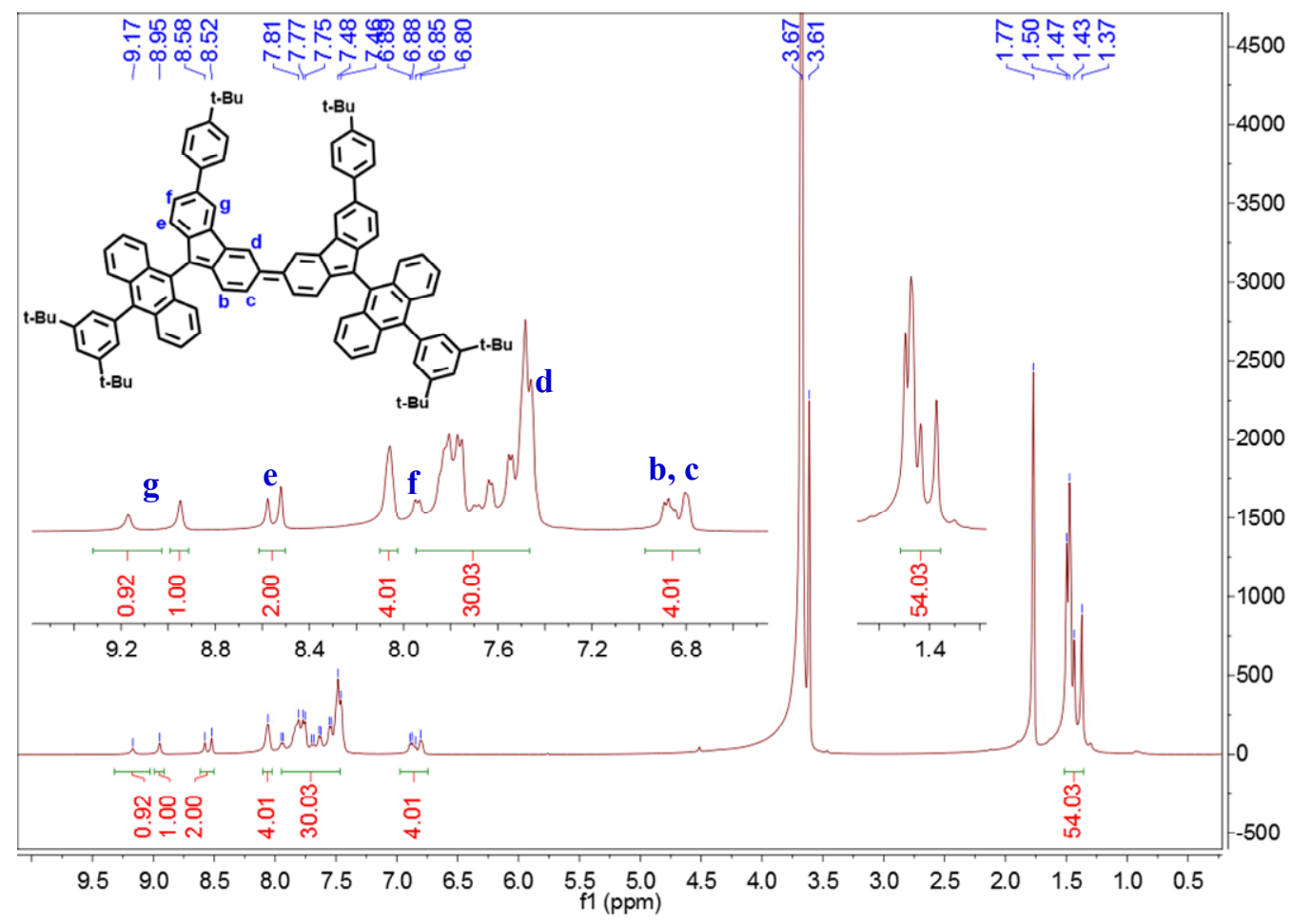

Figure S27. ${ }^{1} \mathrm{H}$ NMR spectrum (500 MHz) of FR-2 in THF- $d_{8}$ at $213 \mathrm{~K}$.

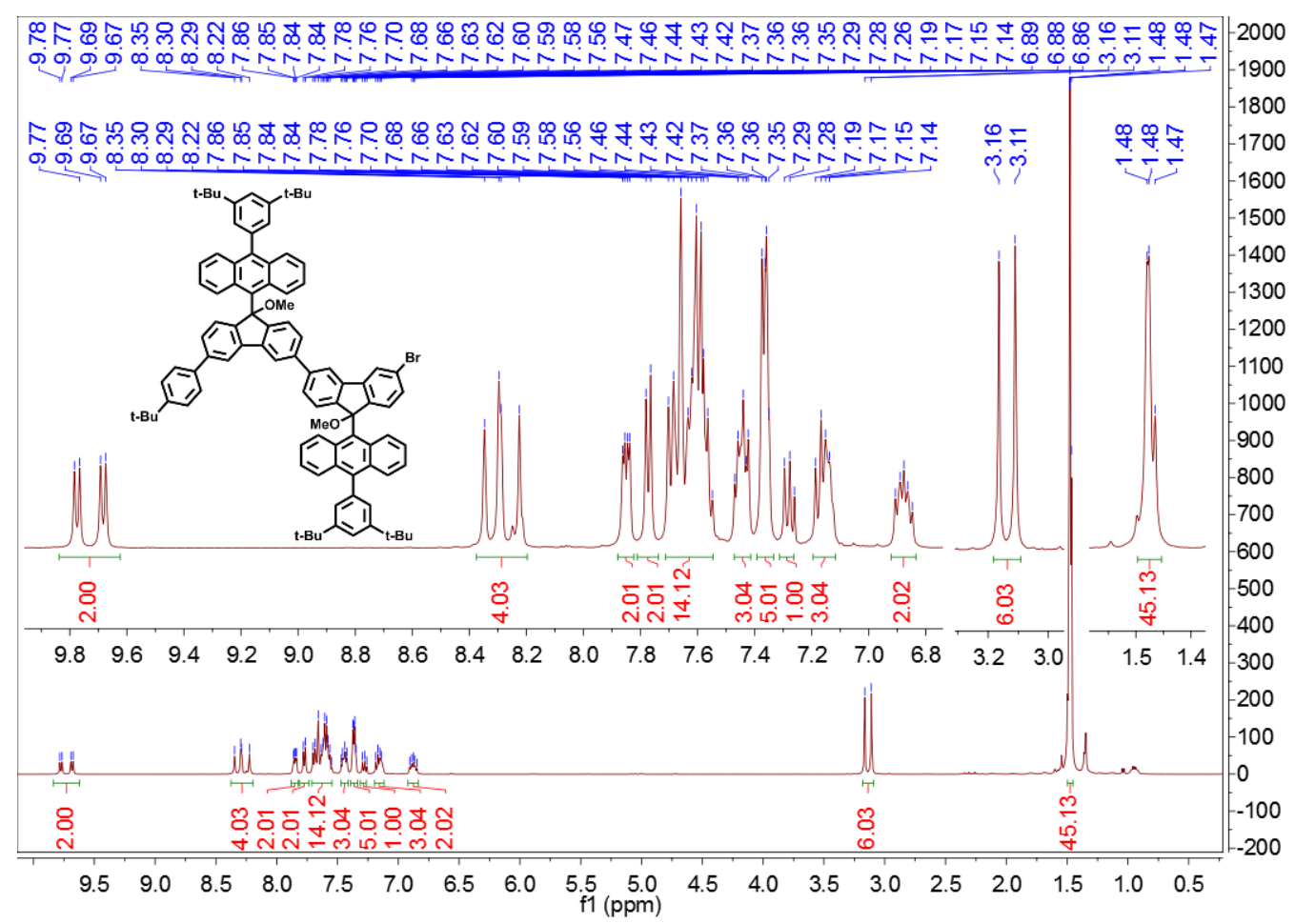

Figure S28. ${ }^{1} \mathrm{H}$ NMR spectrum $(300 \mathrm{MHz})$ of 9 in $\mathrm{CDCl}_{3}$ at $298 \mathrm{~K}$. 


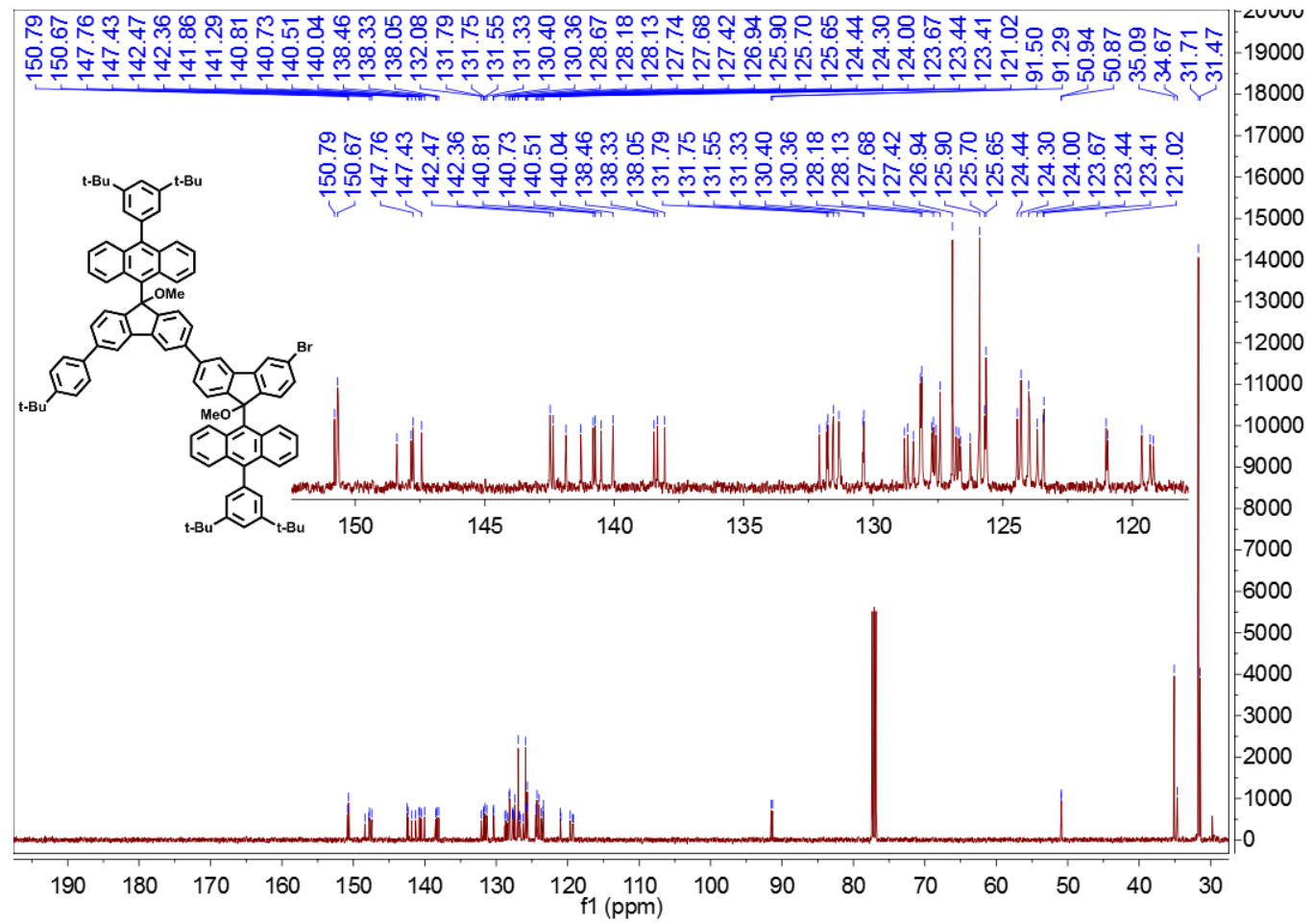

Figure S29. ${ }^{13} \mathrm{C}$ NMR spectrum $(75 \mathrm{MHz})$ of 9 in $\mathrm{CDCl}_{3}$ at $298 \mathrm{~K}$.

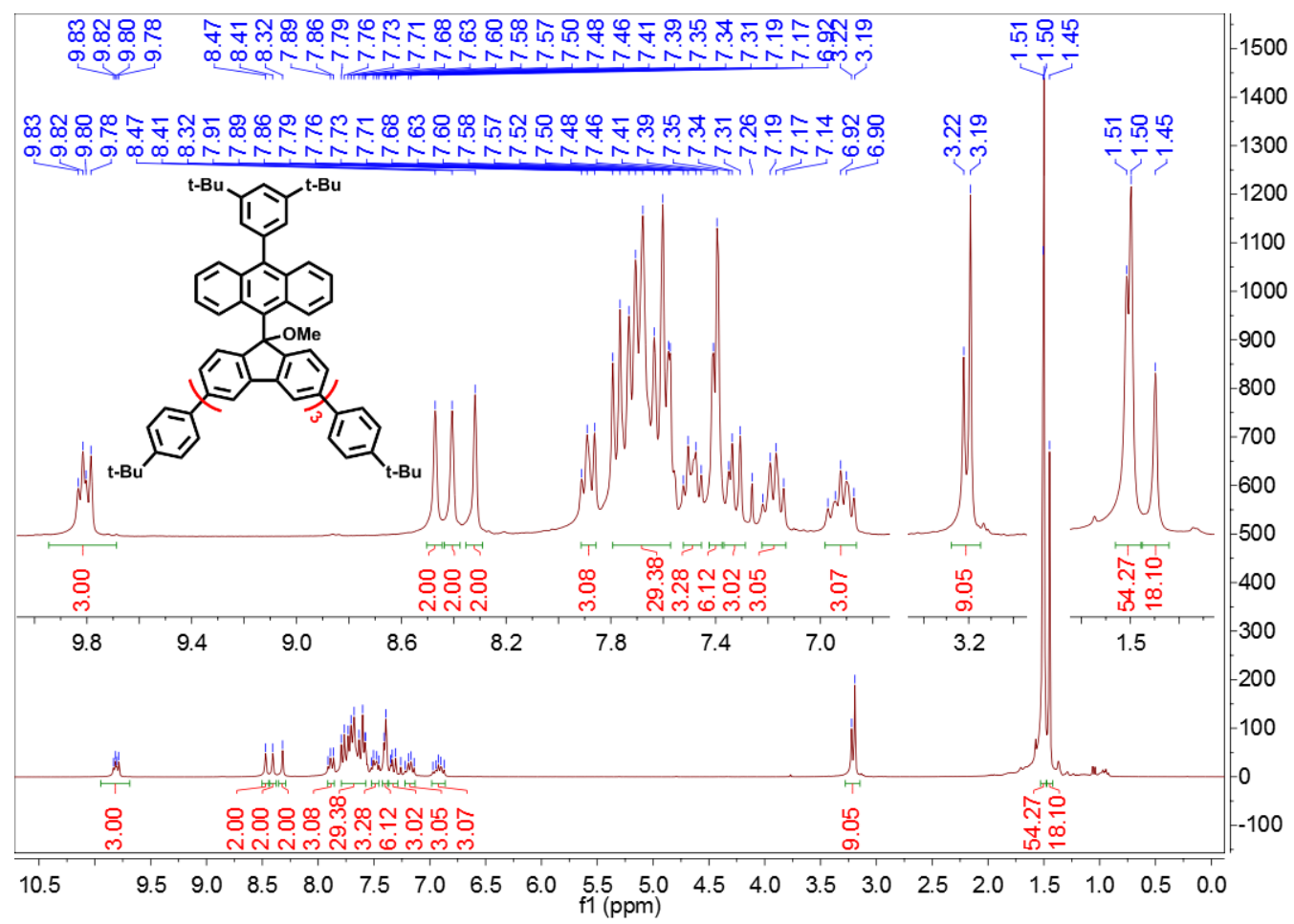

Figure S30. ${ }^{1} \mathrm{H}$ NMR spectrum $(300 \mathrm{MHz})$ of $\mathbf{1 0}$ in $\mathrm{CDCl}_{3}$ at $298 \mathrm{~K}$. 


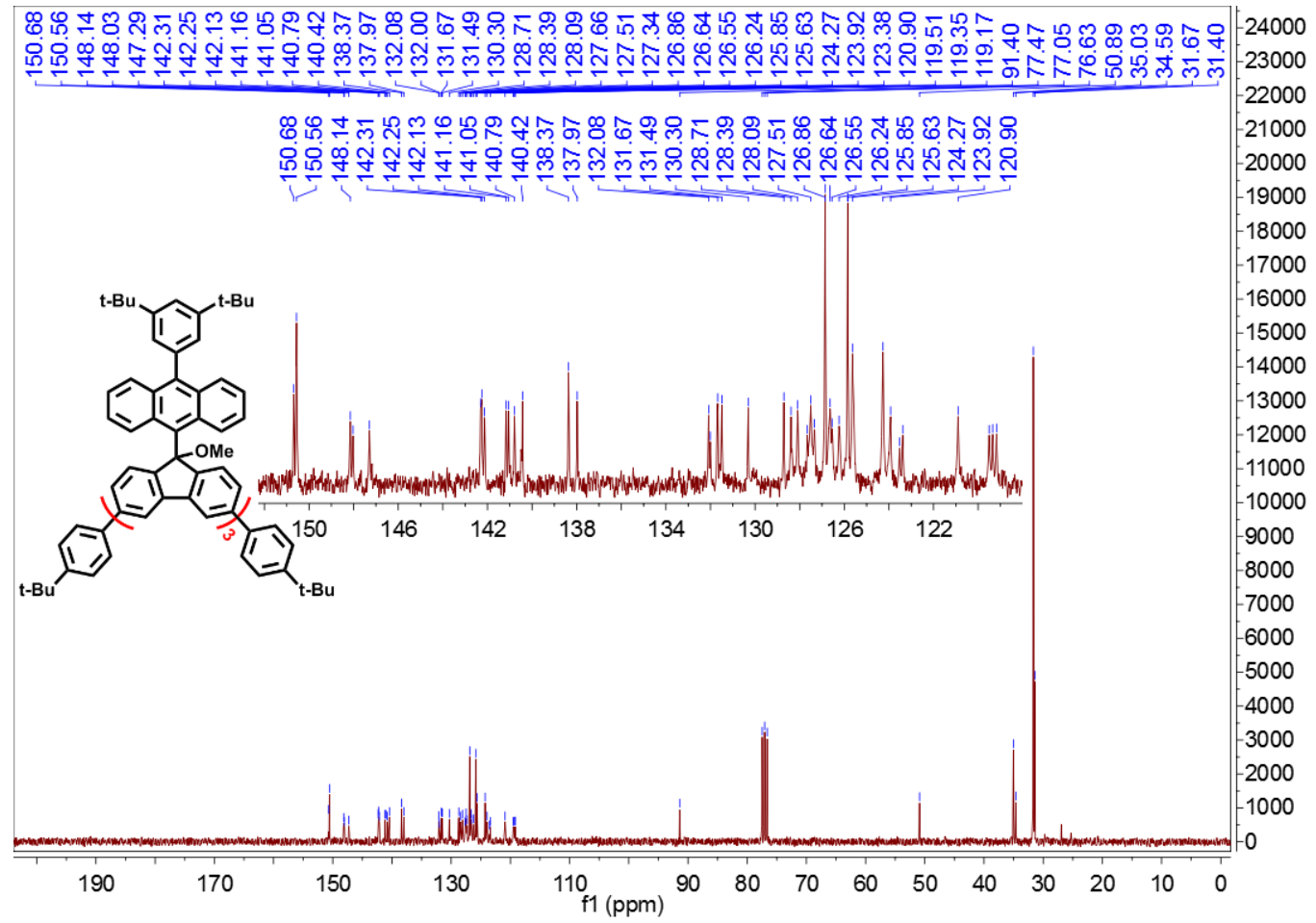

Figure S31. ${ }^{13} \mathrm{C}$ NMR spectrum $(75 \mathrm{MHz})$ of $\mathbf{1 0}$ in $\mathrm{CDCl}_{3}$ at $298 \mathrm{~K}$.

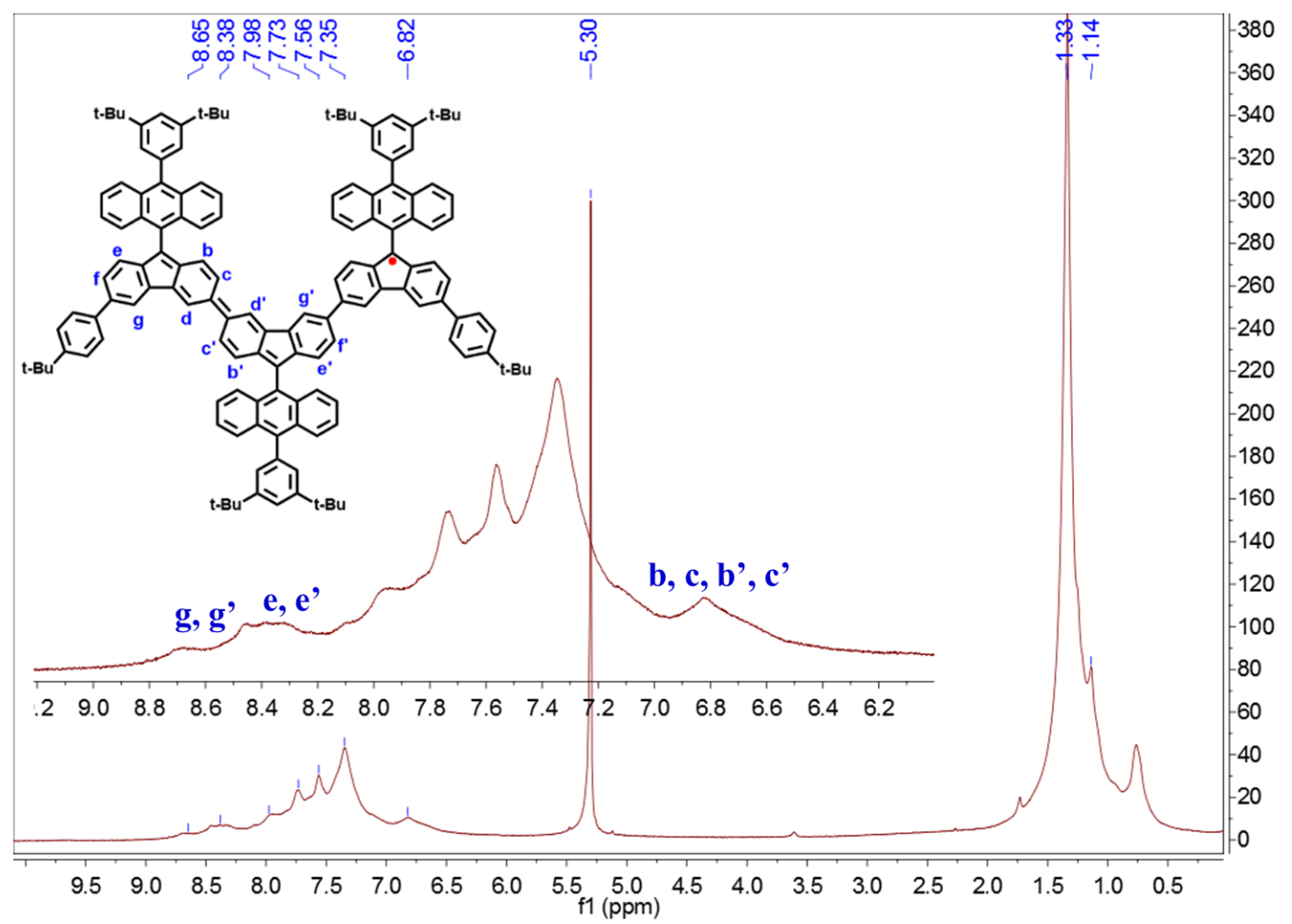

Figure S32. ${ }^{1} \mathrm{H}$ NMR spectrum $(500 \mathrm{MHz})$ of FR-3 in $\mathrm{CD}_{2} \mathrm{Cl}_{2}$ at $213 \mathrm{~K}$. 


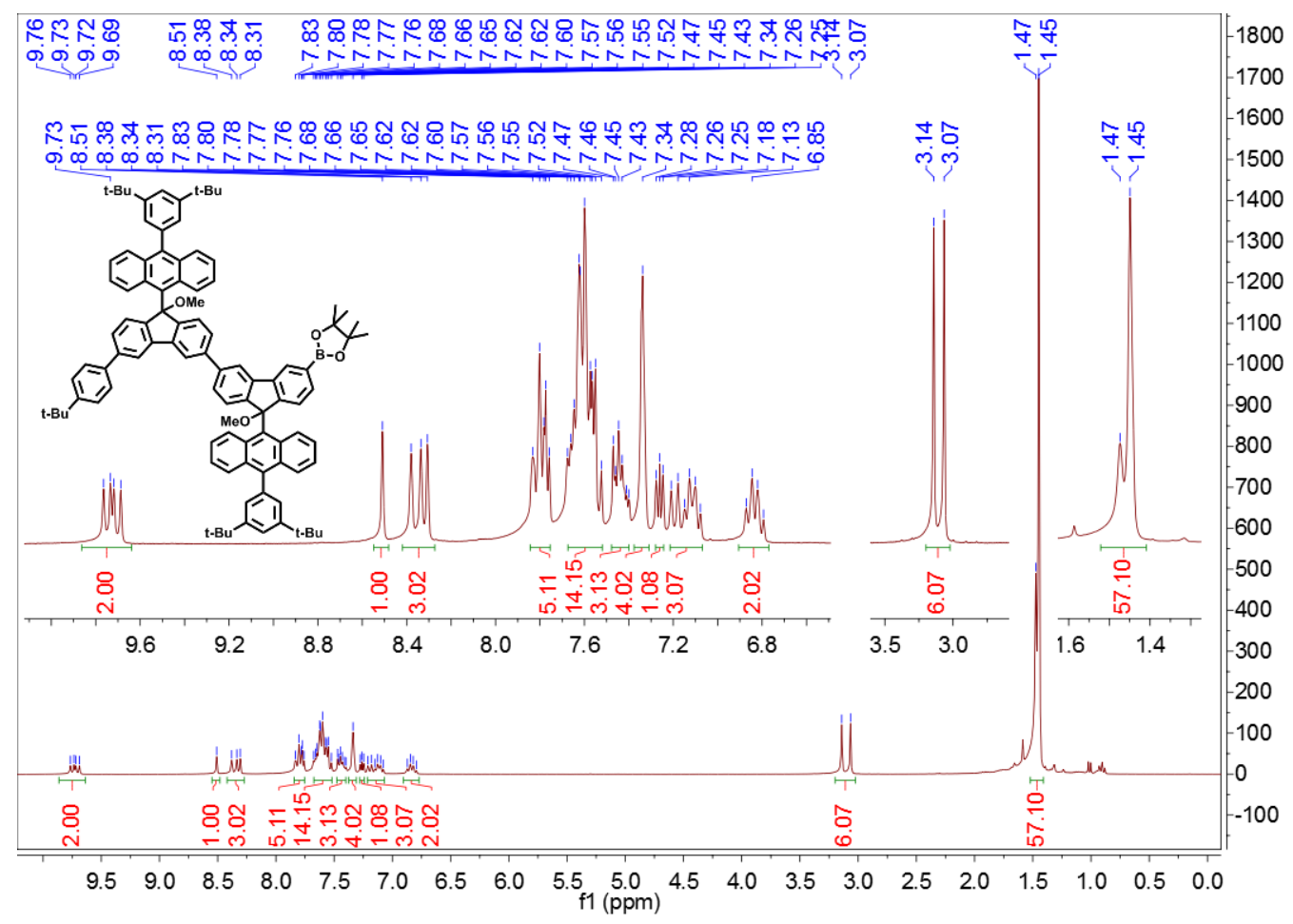

Figure S33. ${ }^{1} \mathrm{H}$ NMR spectrum (300 MHz) of 11 in $\mathrm{CDCl}_{3}$ at $298 \mathrm{~K}$.

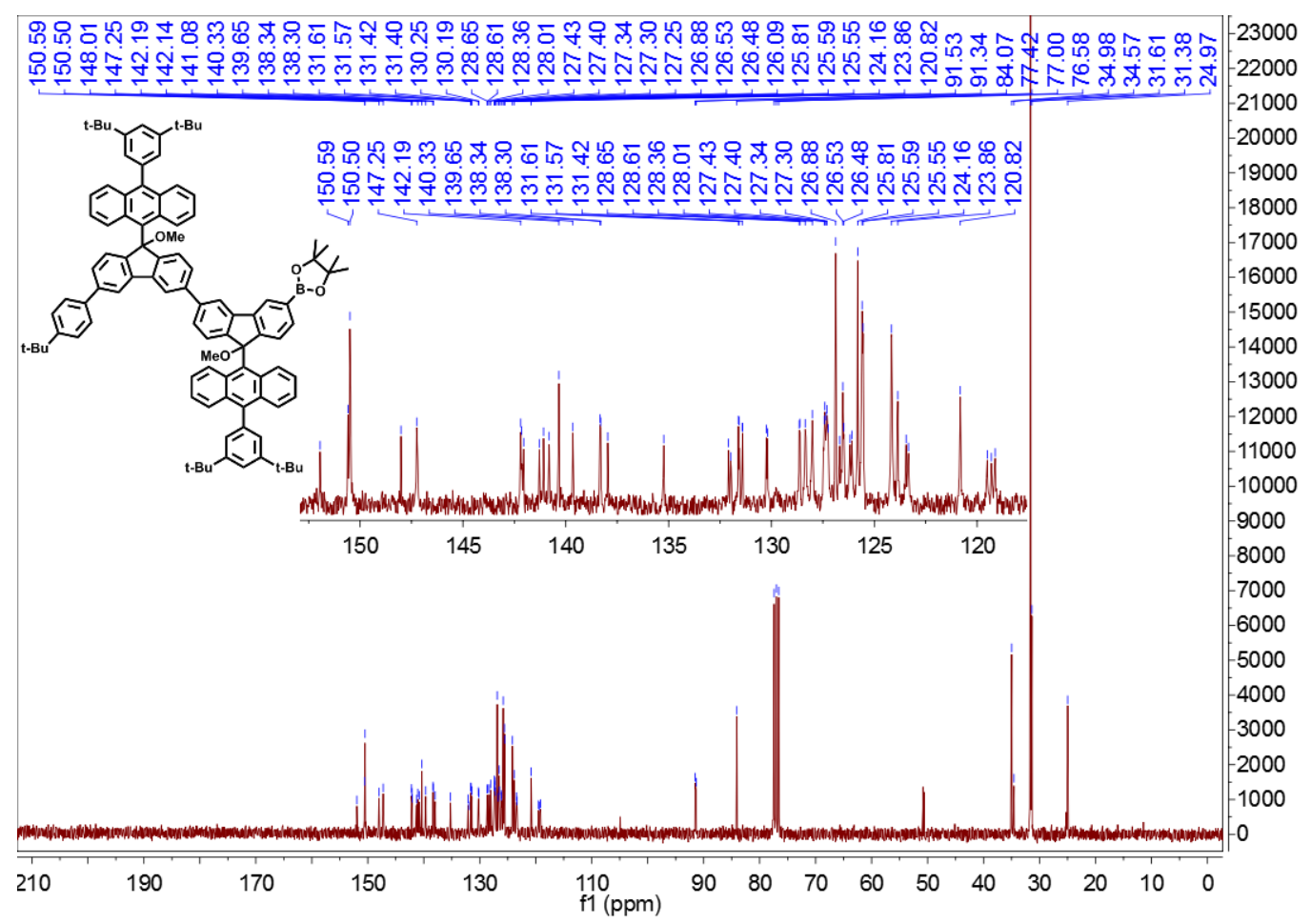

Figure S34. ${ }^{13} \mathrm{C}$ NMR spectrum $(75 \mathrm{MHz})$ of 11 in $\mathrm{CDCl}_{3}$ at $298 \mathrm{~K}$. 


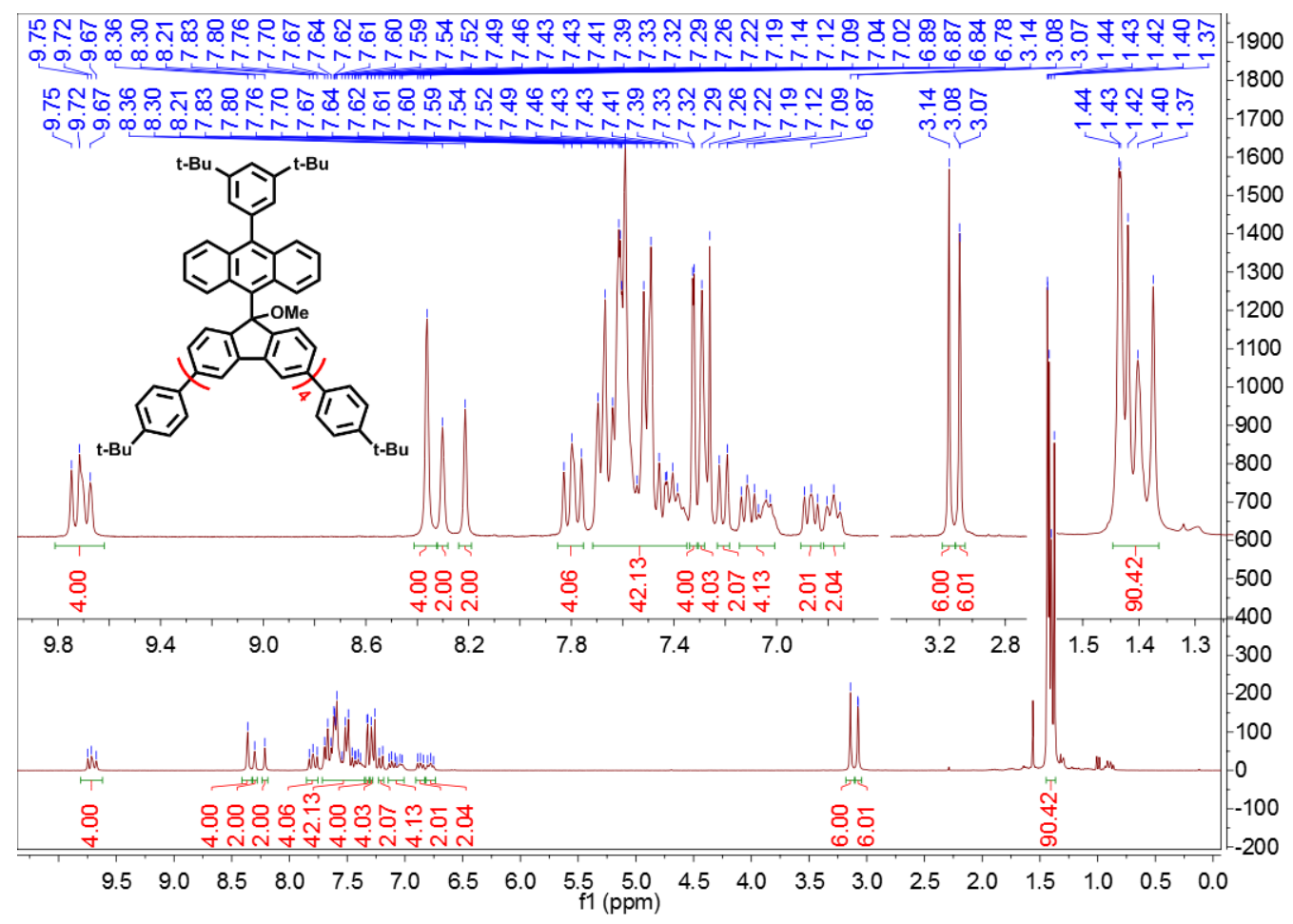

Figure S35. ${ }^{1} \mathrm{H}$ NMR spectrum (300 MHz) of 12 in $\mathrm{CDCl}_{3}$ at $298 \mathrm{~K}$.

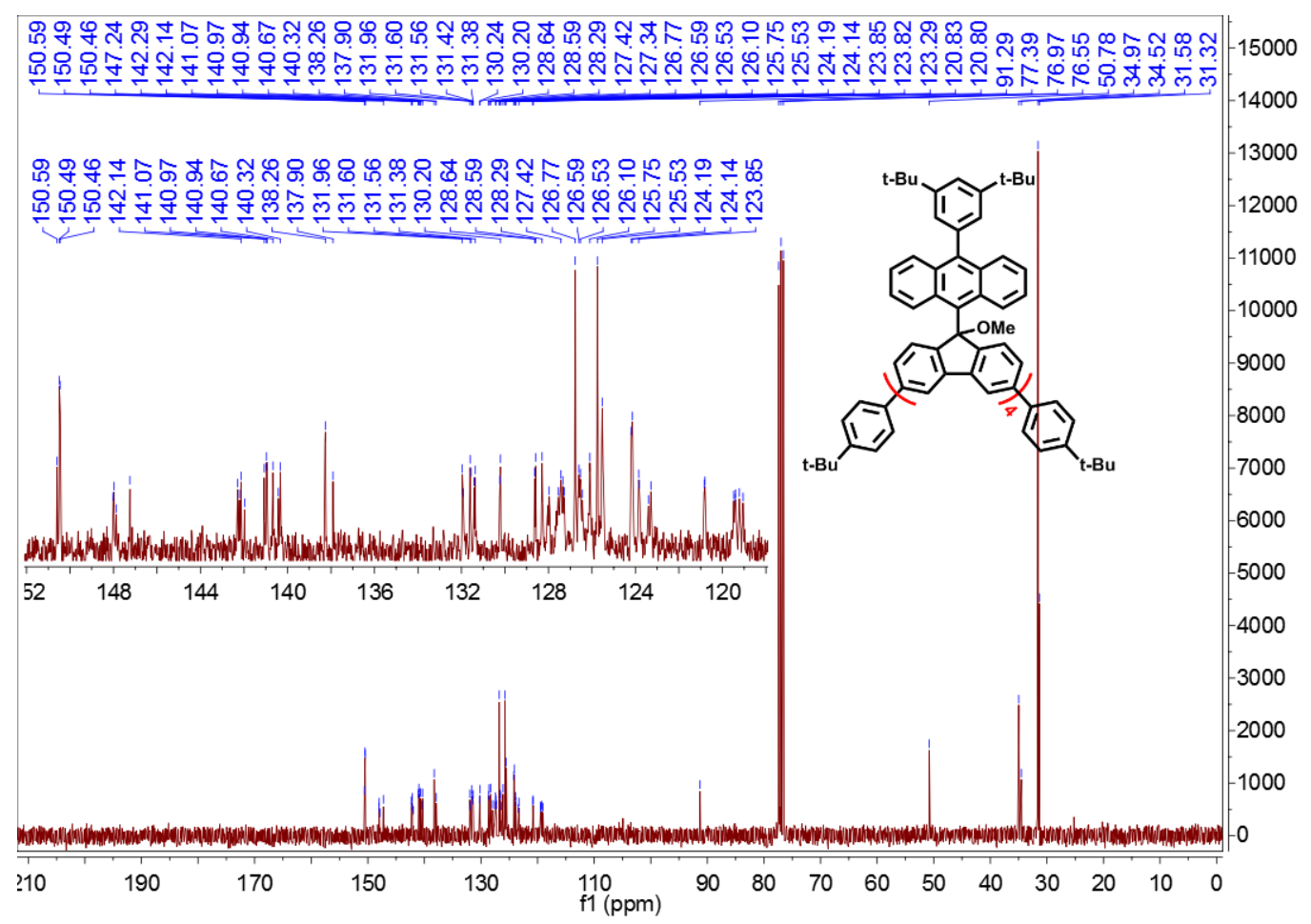

Figure S36. ${ }^{13} \mathrm{C}$ NMR spectrum $(75 \mathrm{MHz})$ of 12 in $\mathrm{CDCl}_{3}$ at $298 \mathrm{~K}$. 


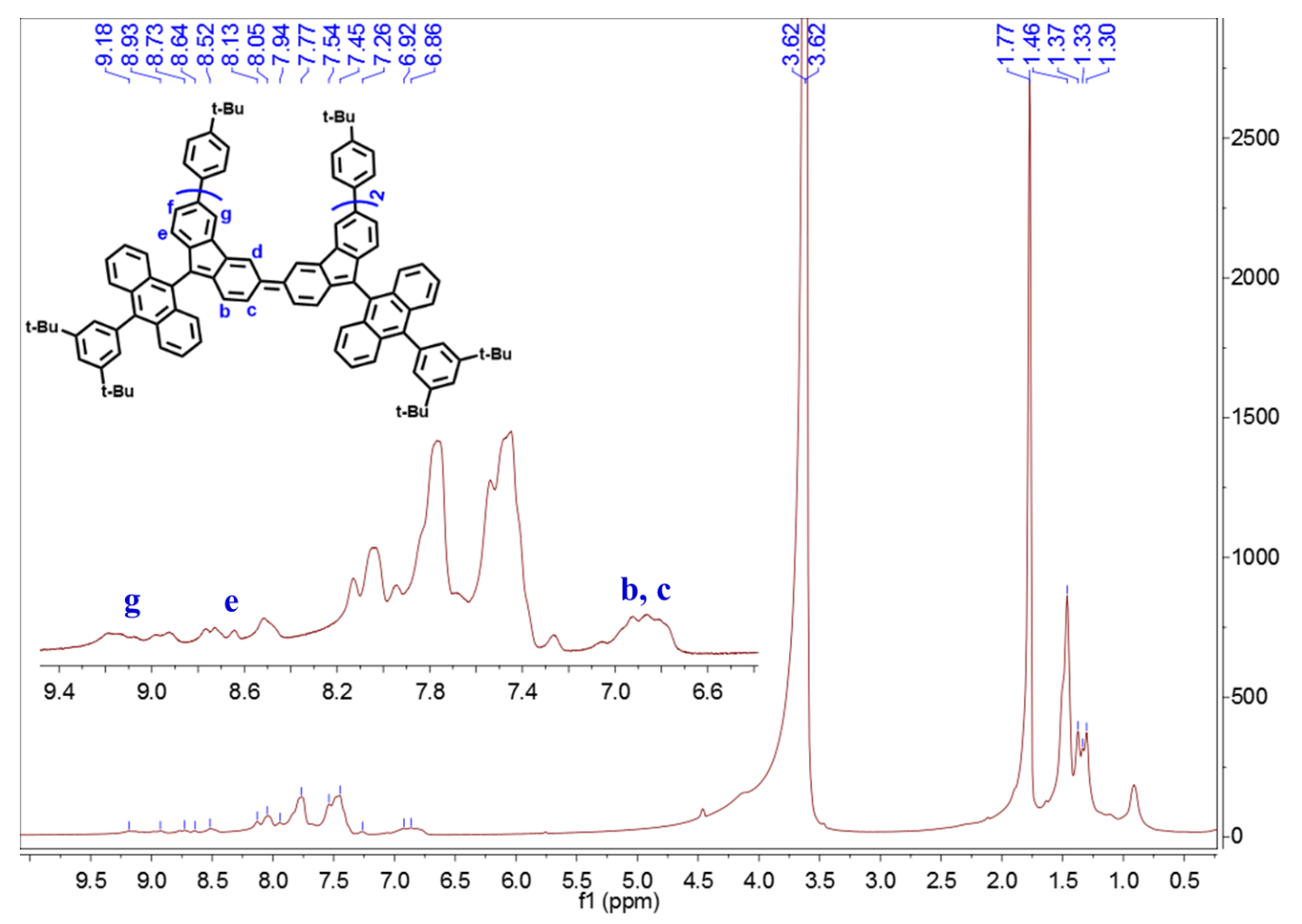

Figure S37. ${ }^{1} \mathrm{H}$ NMR spectrum $(500 \mathrm{MHz})$ of FR-4 in THF- $d_{8}$ at $213 \mathrm{~K}$.

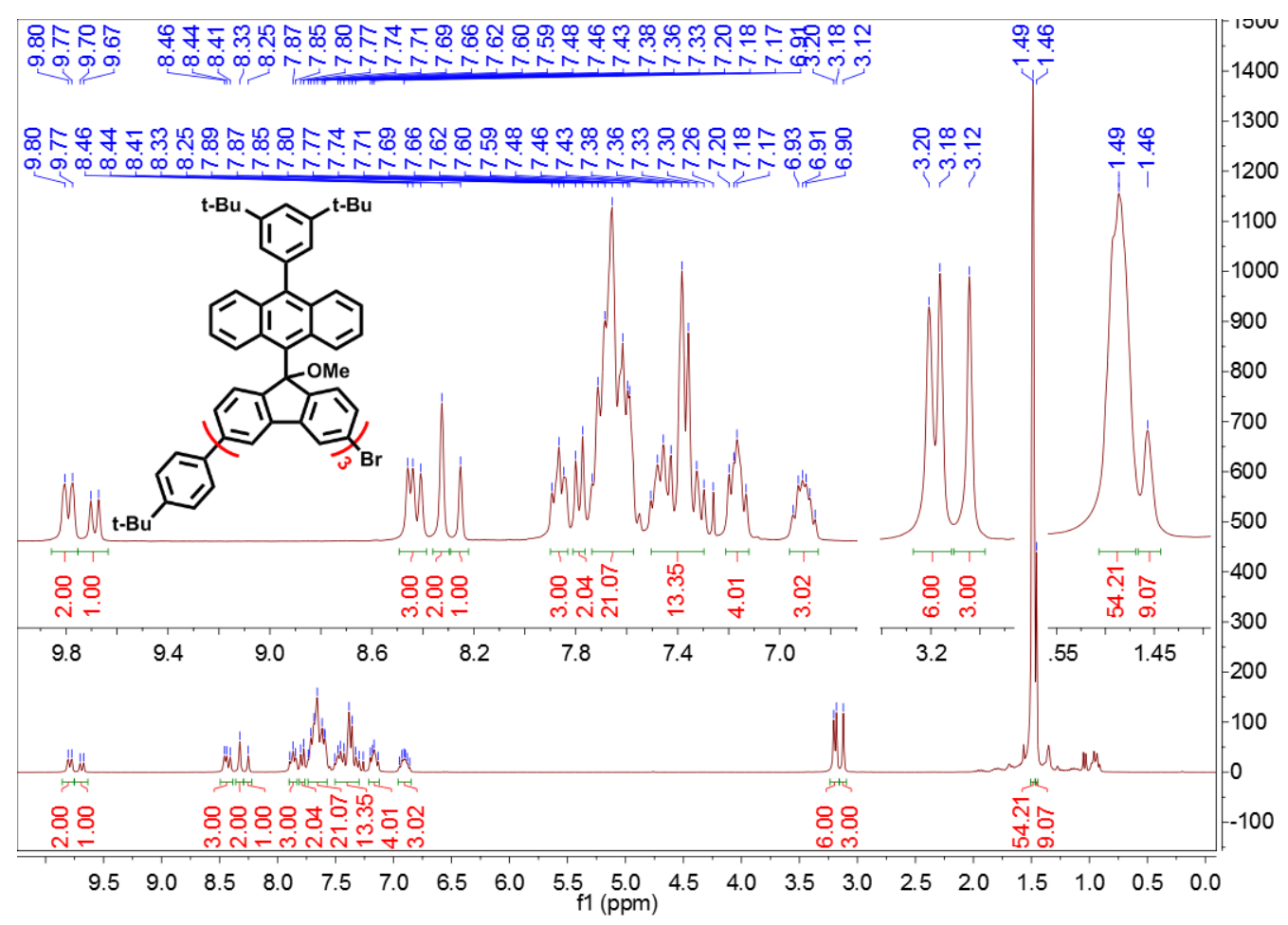

Figure S38. ${ }^{1} \mathrm{H}$ NMR spectrum (300 MHz) of 13 in $\mathrm{CDCl}_{3}$ at $298 \mathrm{~K}$. 


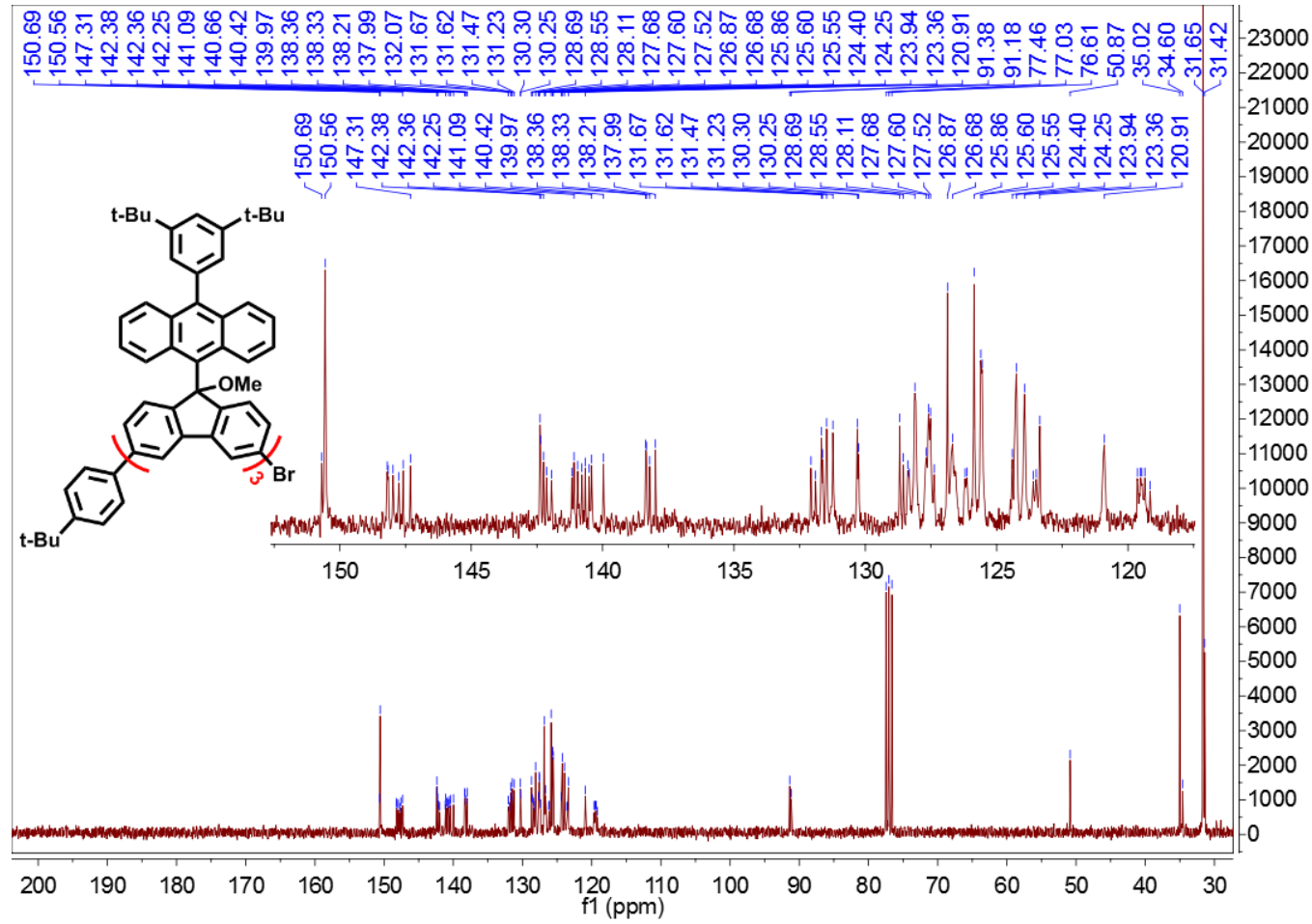

Figure S39. ${ }^{13} \mathrm{C}$ NMR spectrum $(75 \mathrm{MHz})$ of 13 in $\mathrm{CDCl}_{3}$ at $298 \mathrm{~K}$.

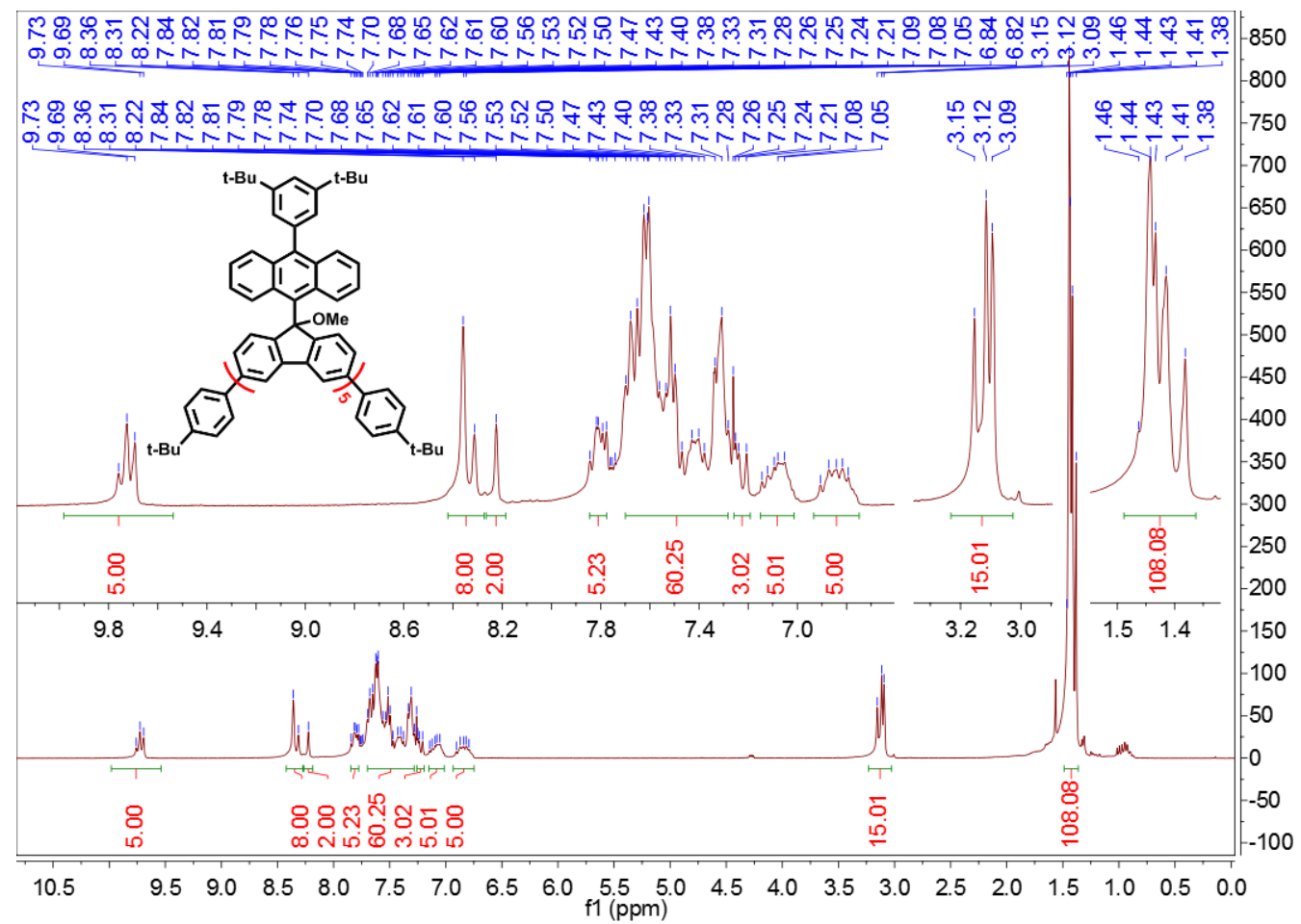

Figure S40. ${ }^{1} \mathrm{H}$ NMR spectrum $\left(300 \mathrm{MHz}\right.$ ) of $\mathbf{1 4}$ in $\mathrm{CDCl}_{3}$ at $298 \mathrm{~K}$. 


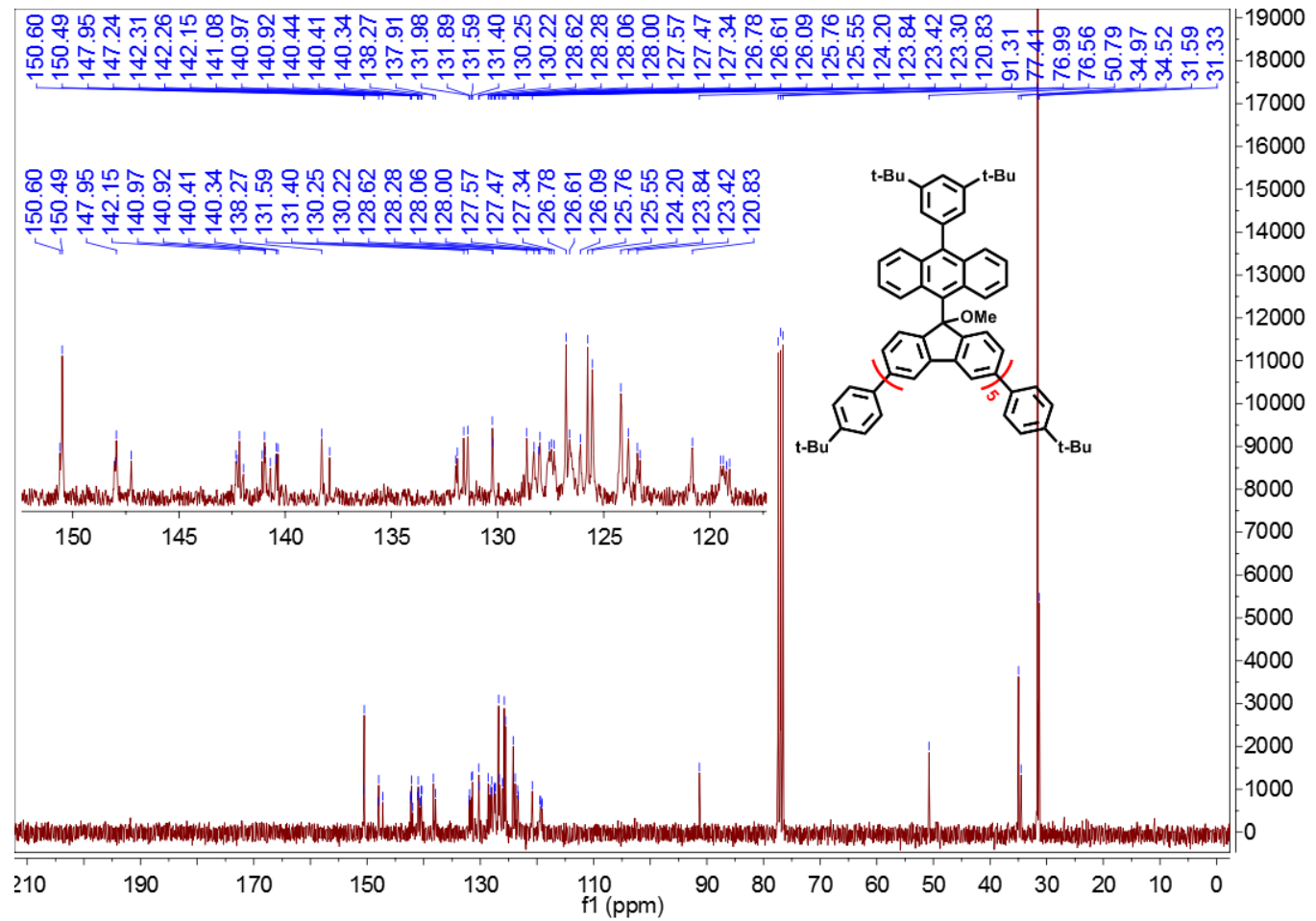

Figure S41. ${ }^{13} \mathrm{C}$ NMR spectrum $(75 \mathrm{MHz})$ of 14 in $\mathrm{CDCl}_{3}$ at $298 \mathrm{~K}$.

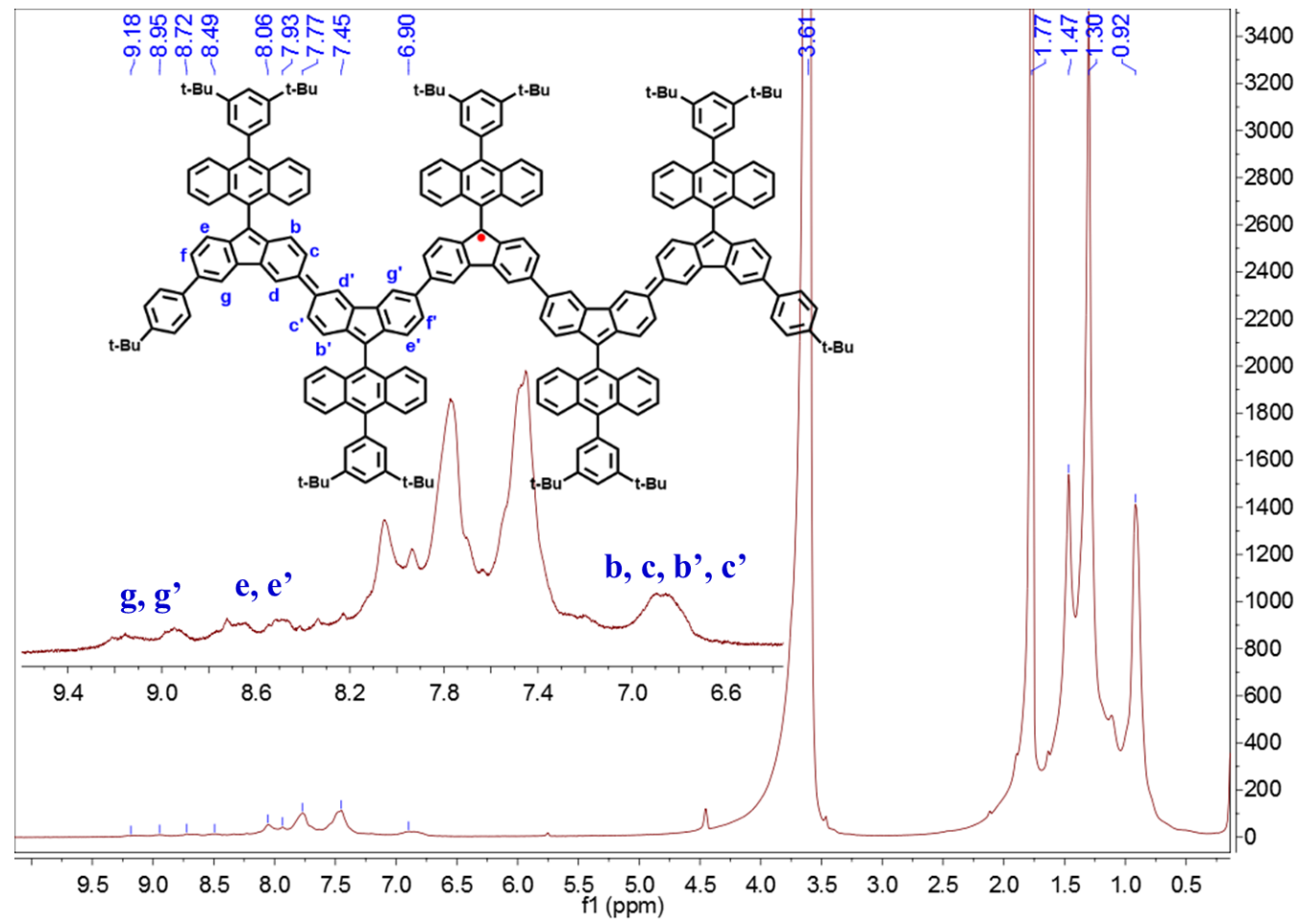

Figure S42. ${ }^{1} \mathrm{H}$ NMR spectrum $(500 \mathrm{MHz})$ of FR-5 in THF- $d_{8}$ at $213 \mathrm{~K}$. 


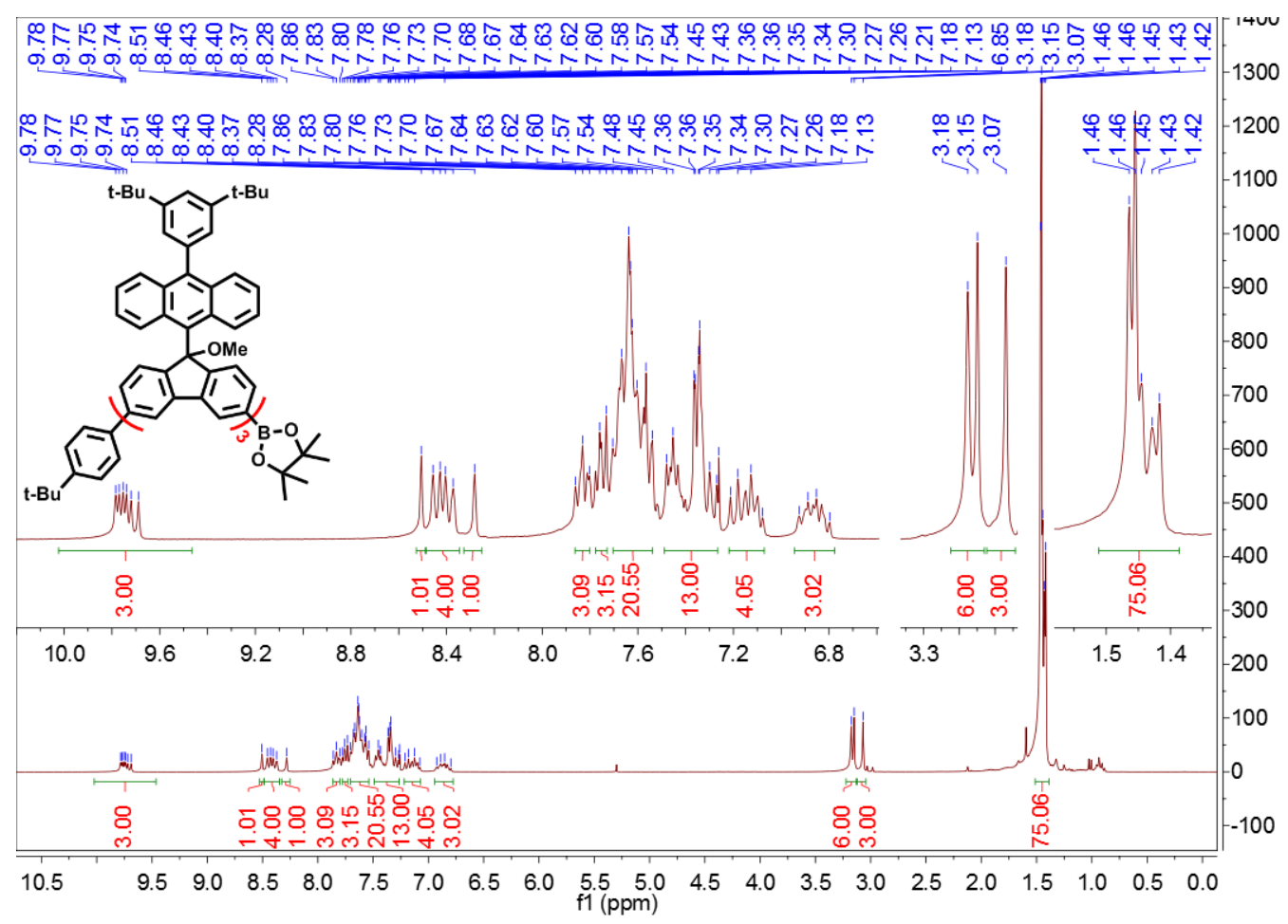

Figure S43. ${ }^{1} \mathrm{H}$ NMR spectrum (300 MHz) of 15 in $\mathrm{CDCl}_{3}$ at $298 \mathrm{~K}$.

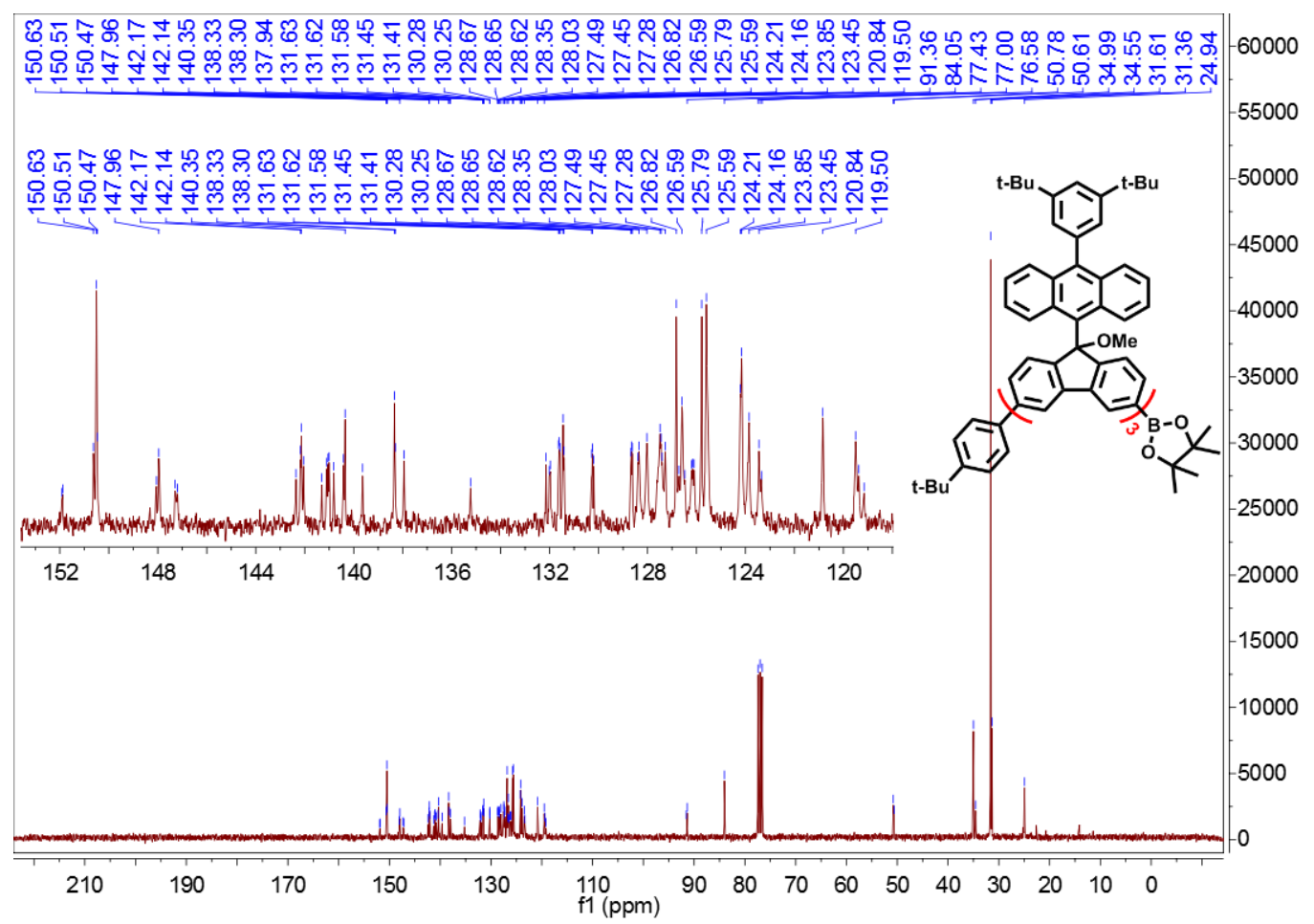

Figure S44. ${ }^{13} \mathrm{C}$ NMR spectrum $(75 \mathrm{MHz})$ of $\mathbf{1 5}$ in $\mathrm{CDCl}_{3}$ at $298 \mathrm{~K}$. 


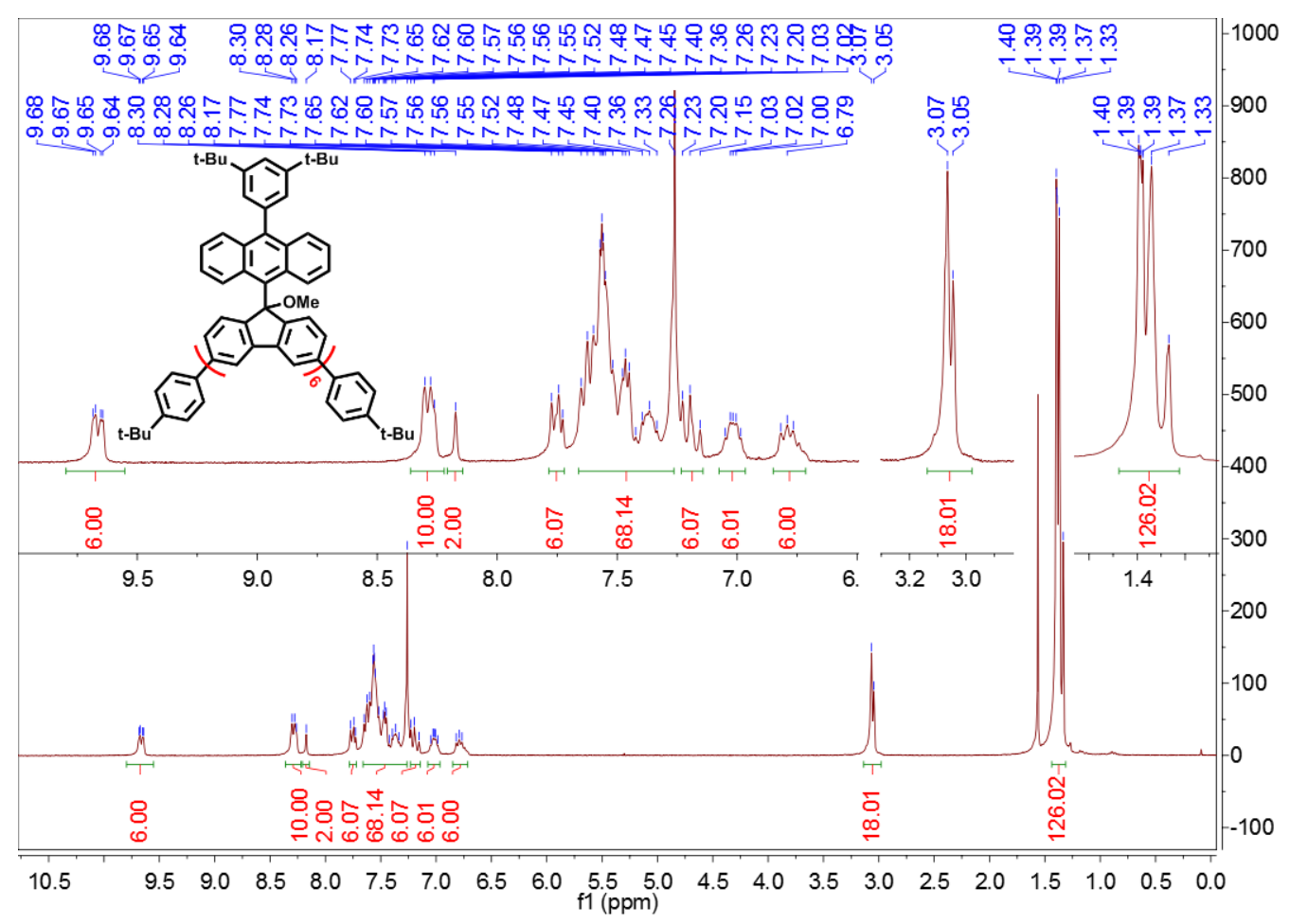

Figure S45. ${ }^{1} \mathrm{H}$ NMR spectrum (300 MHz) of 16 in $\mathrm{CDCl}_{3}$ at $298 \mathrm{~K}$.

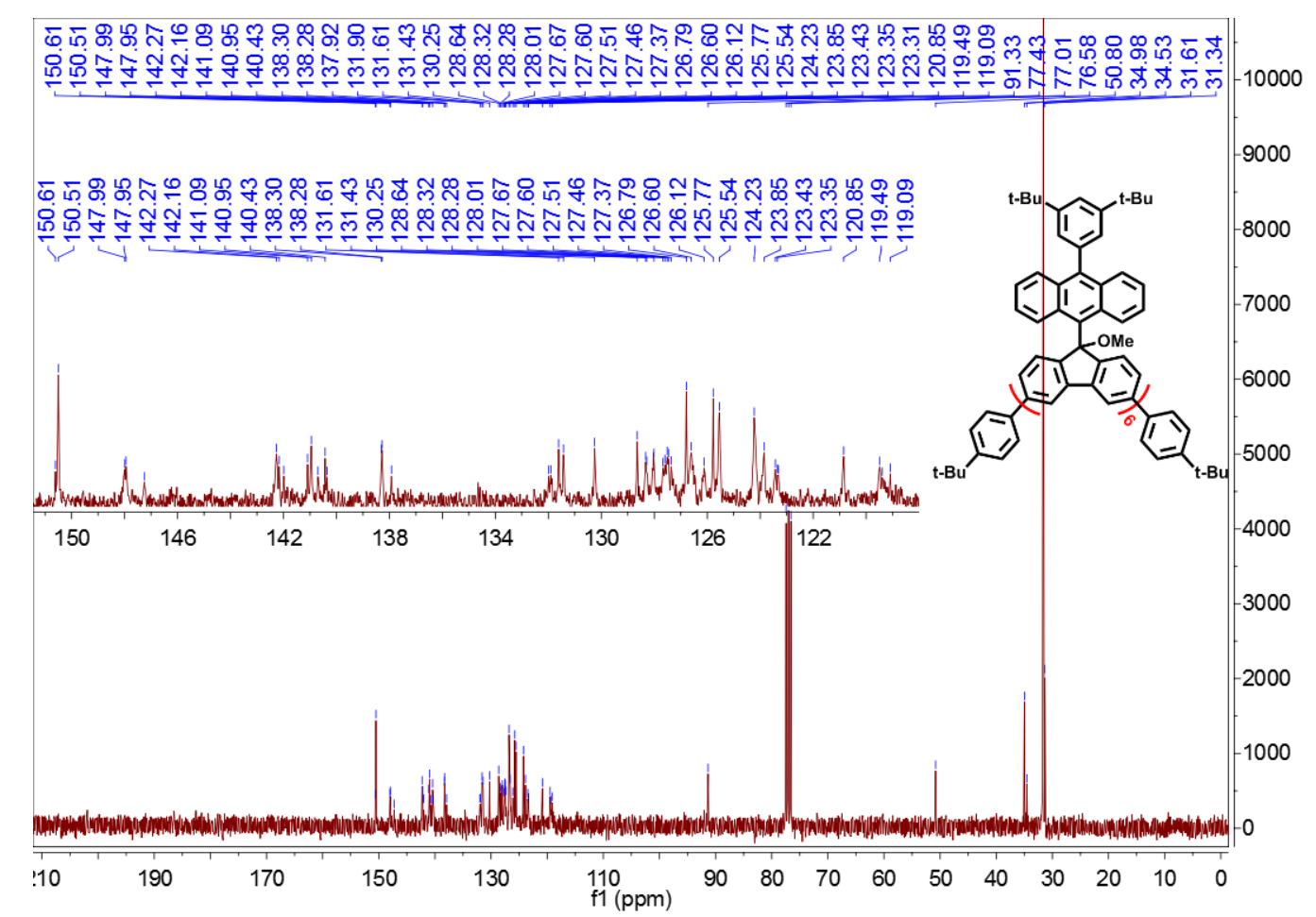

Figure S46. ${ }^{13} \mathrm{C}$ NMR spectrum $(75 \mathrm{MHz})$ of 16 in $\mathrm{CDCl}_{3}$ at $298 \mathrm{~K}$. 


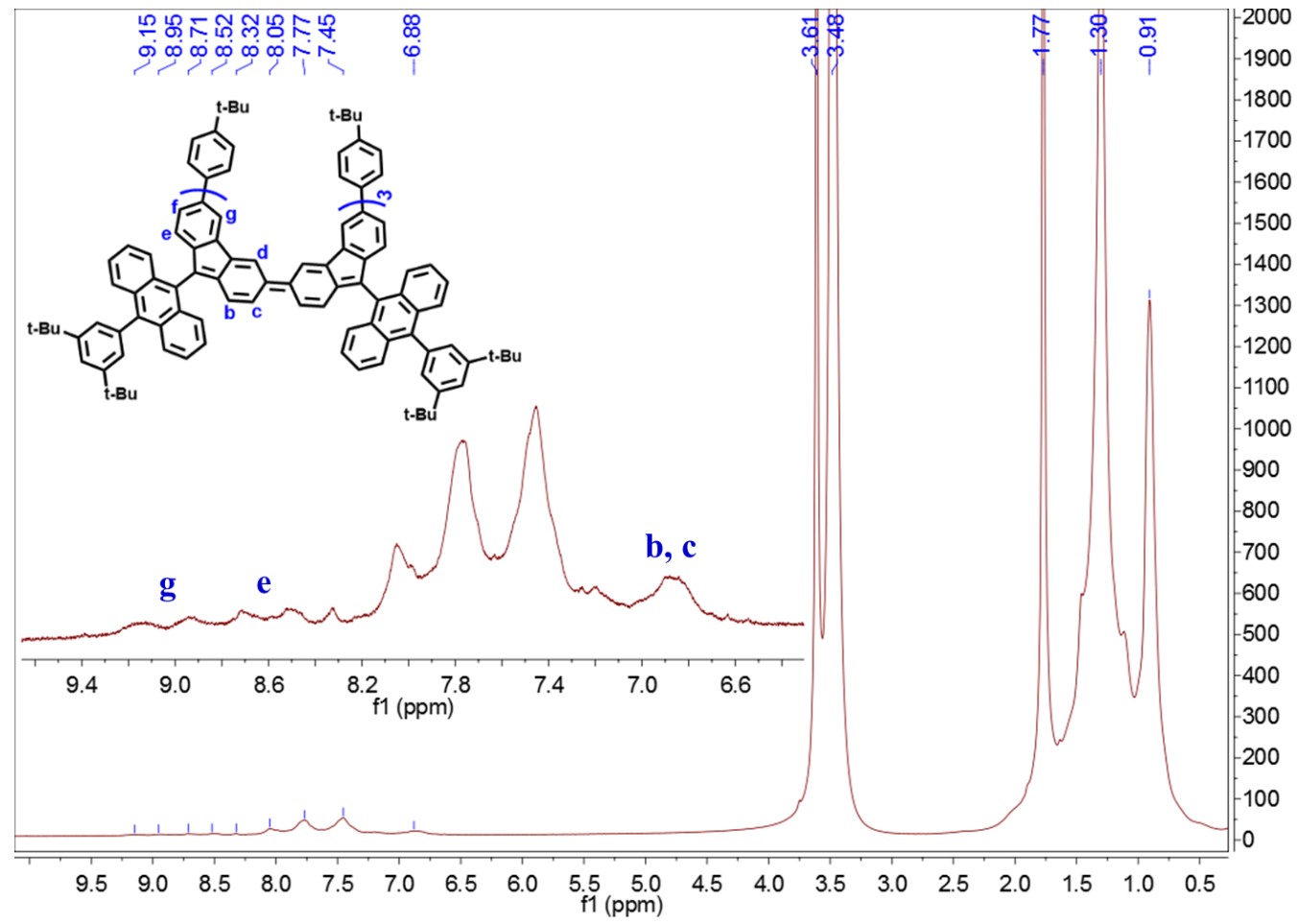

Figure S47. ${ }^{1} \mathrm{H}$ NMR spectrum $(500 \mathrm{MHz})$ of FR-5 in THF- $d_{8}$ at $213 \mathrm{~K}$.

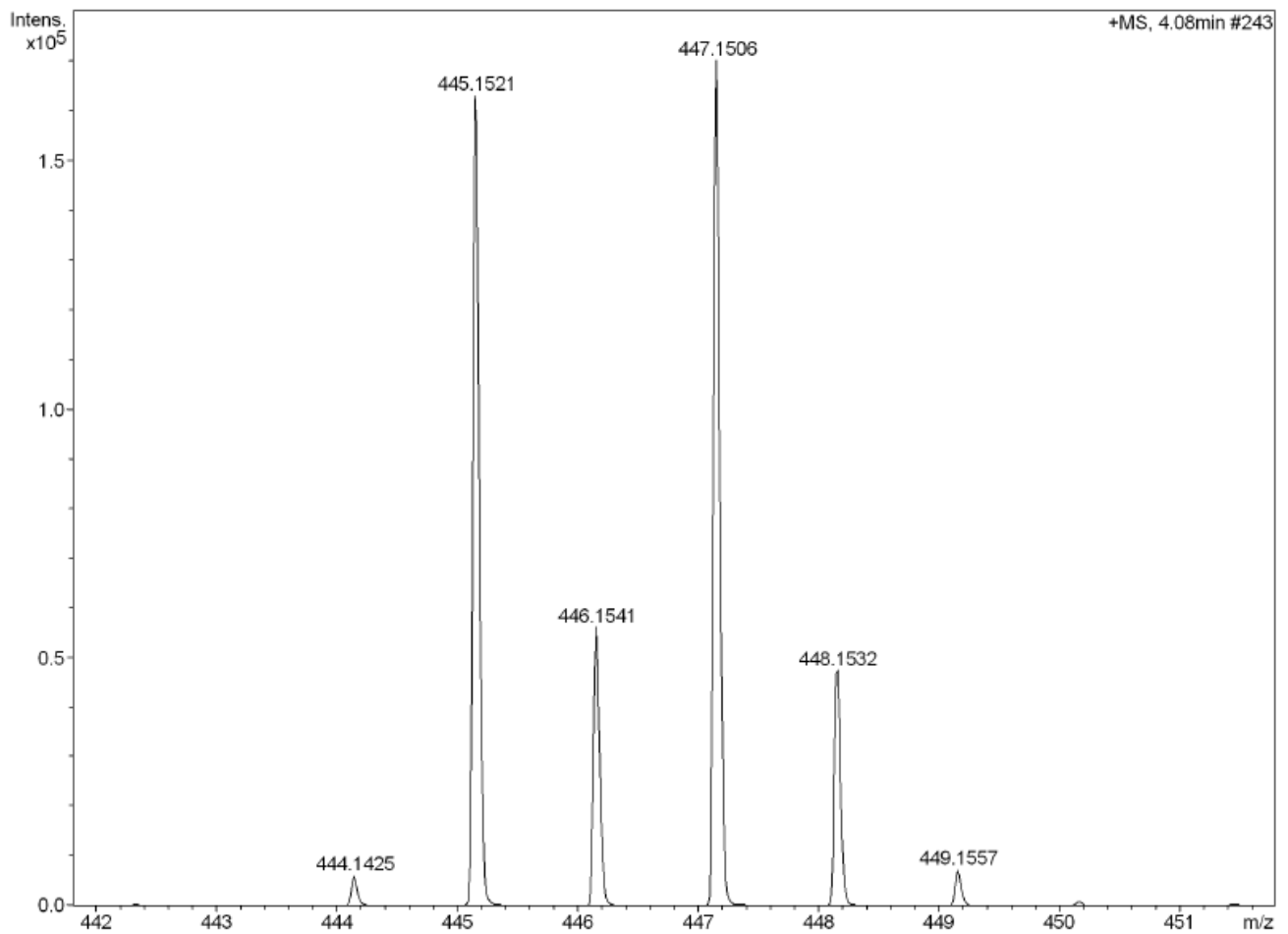

Figure S48. HR mass spectrum (APCI) of compound 2. 


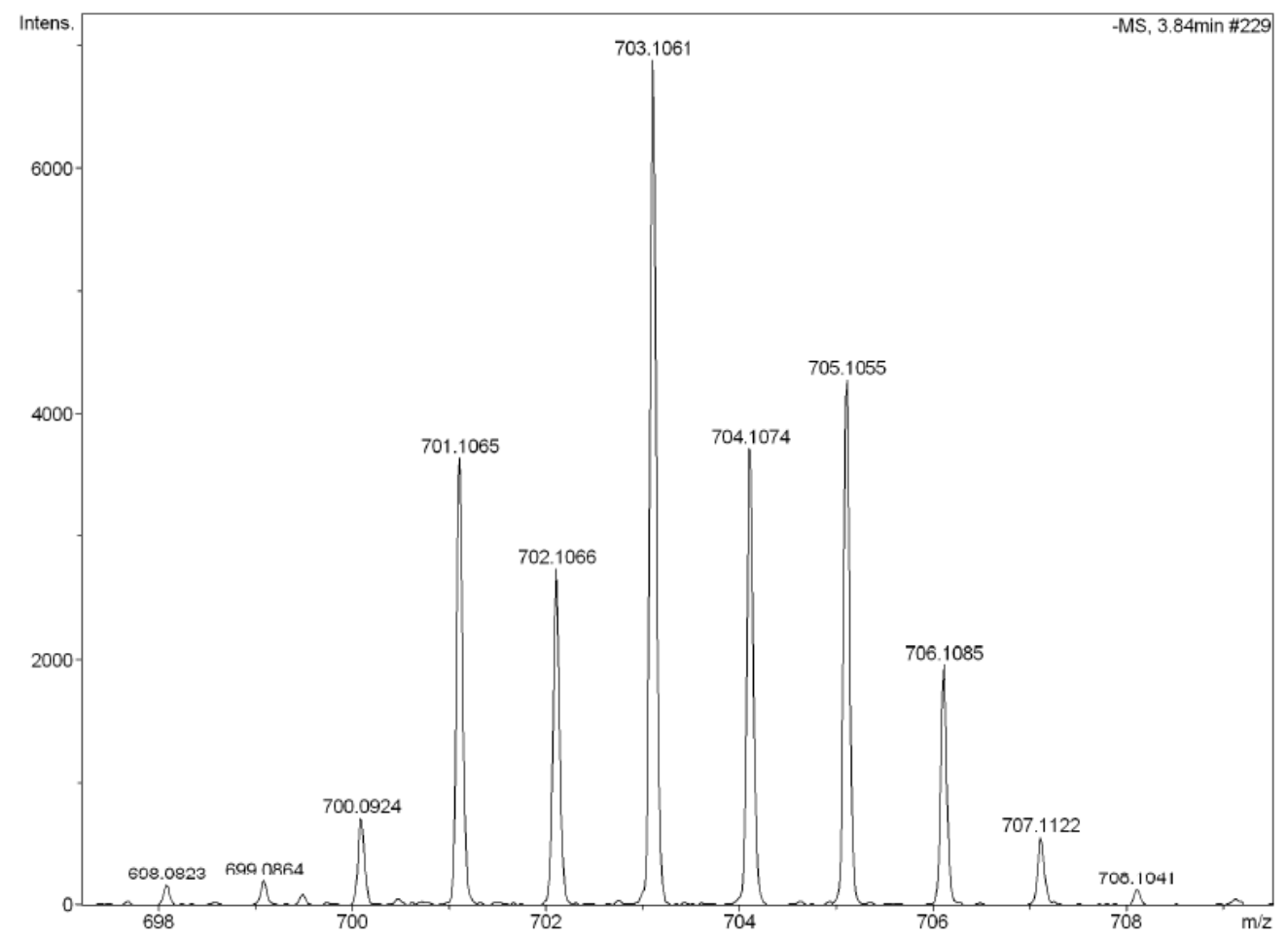

Figure S49. HR mass spectrum (APCI) of compound 3.

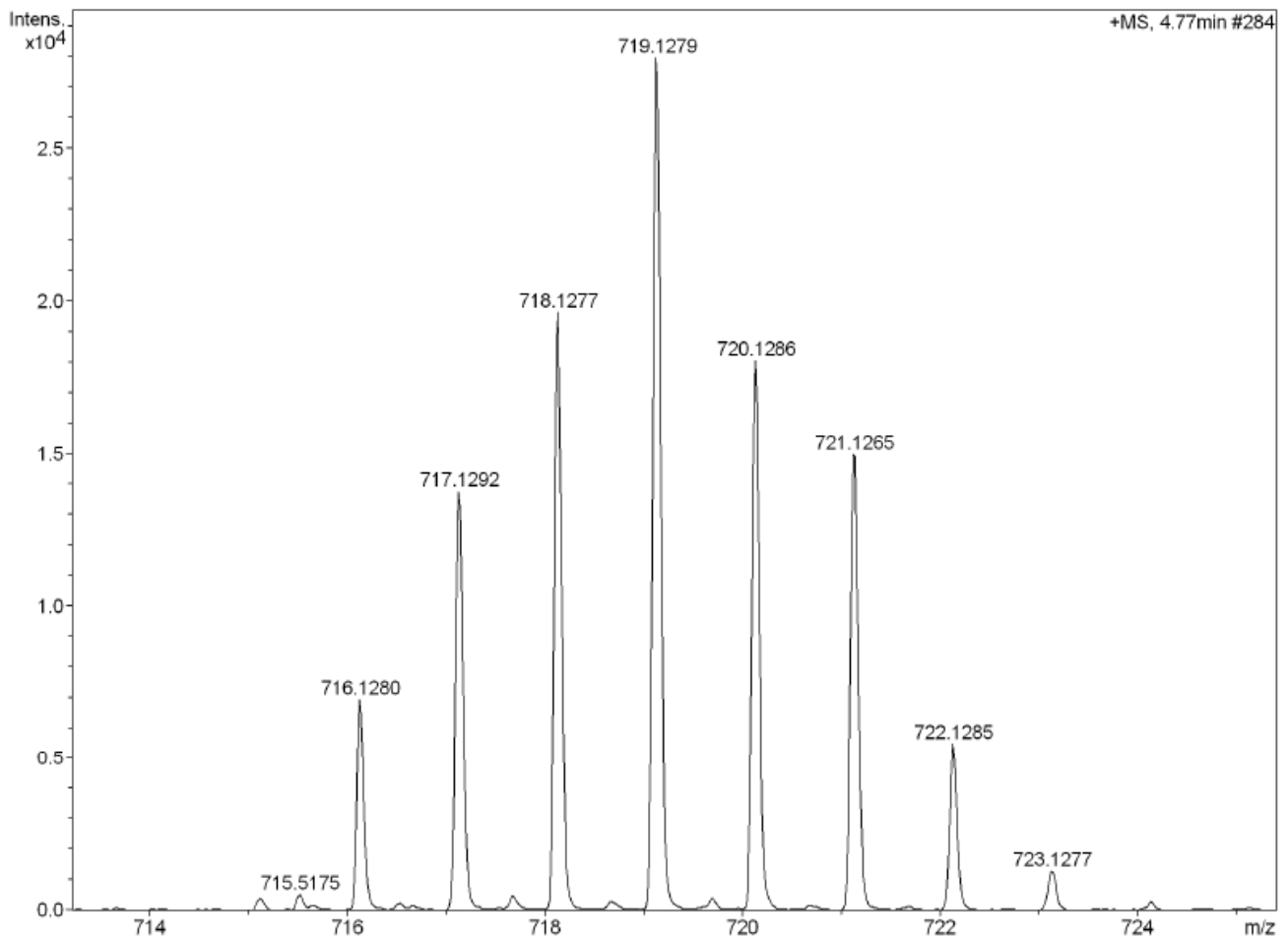

Figure S50. HR mass spectrum (APCI) of compound 4. 


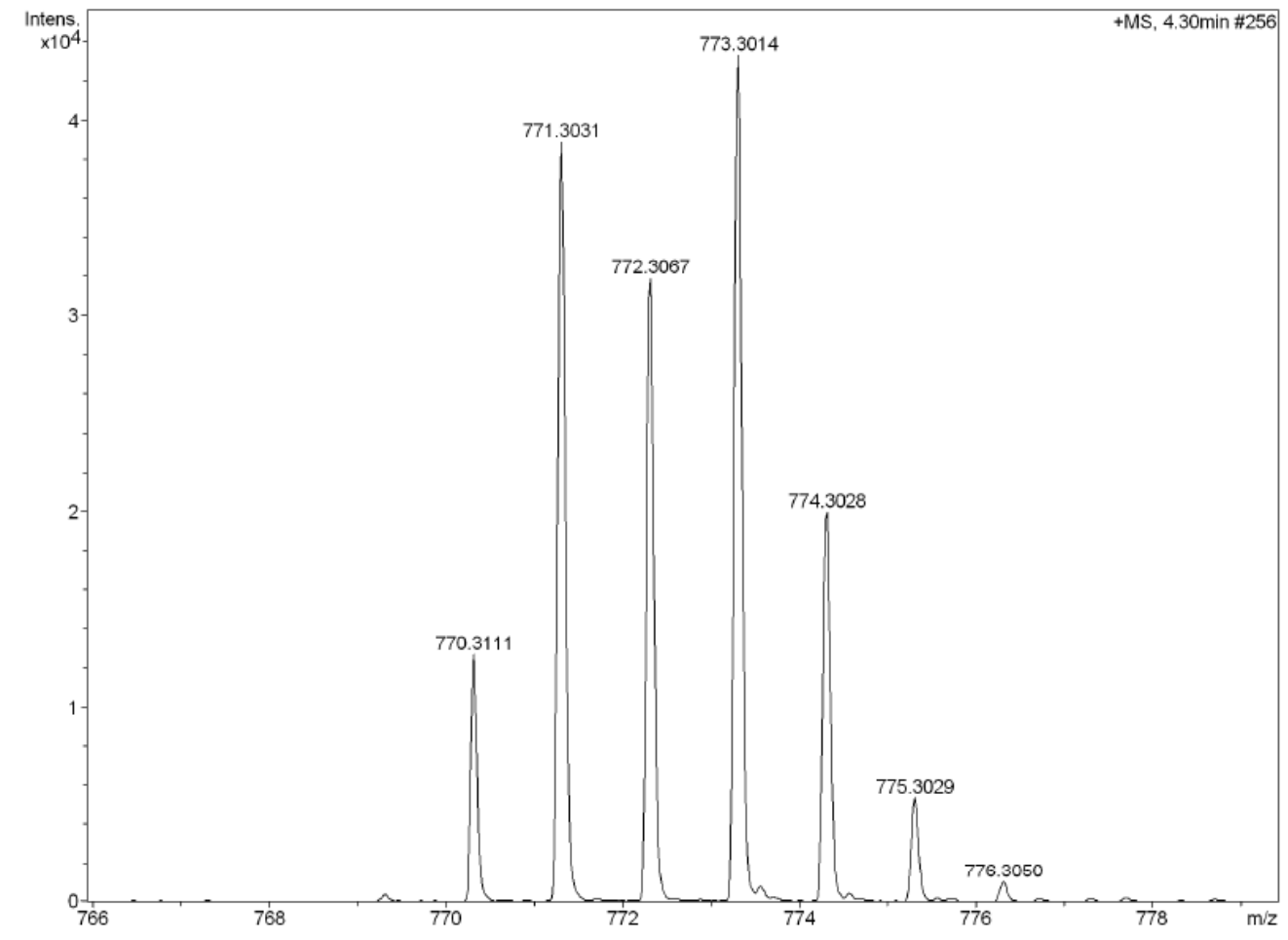

Figure S51. HR mass spectrum (APCI) of 5.

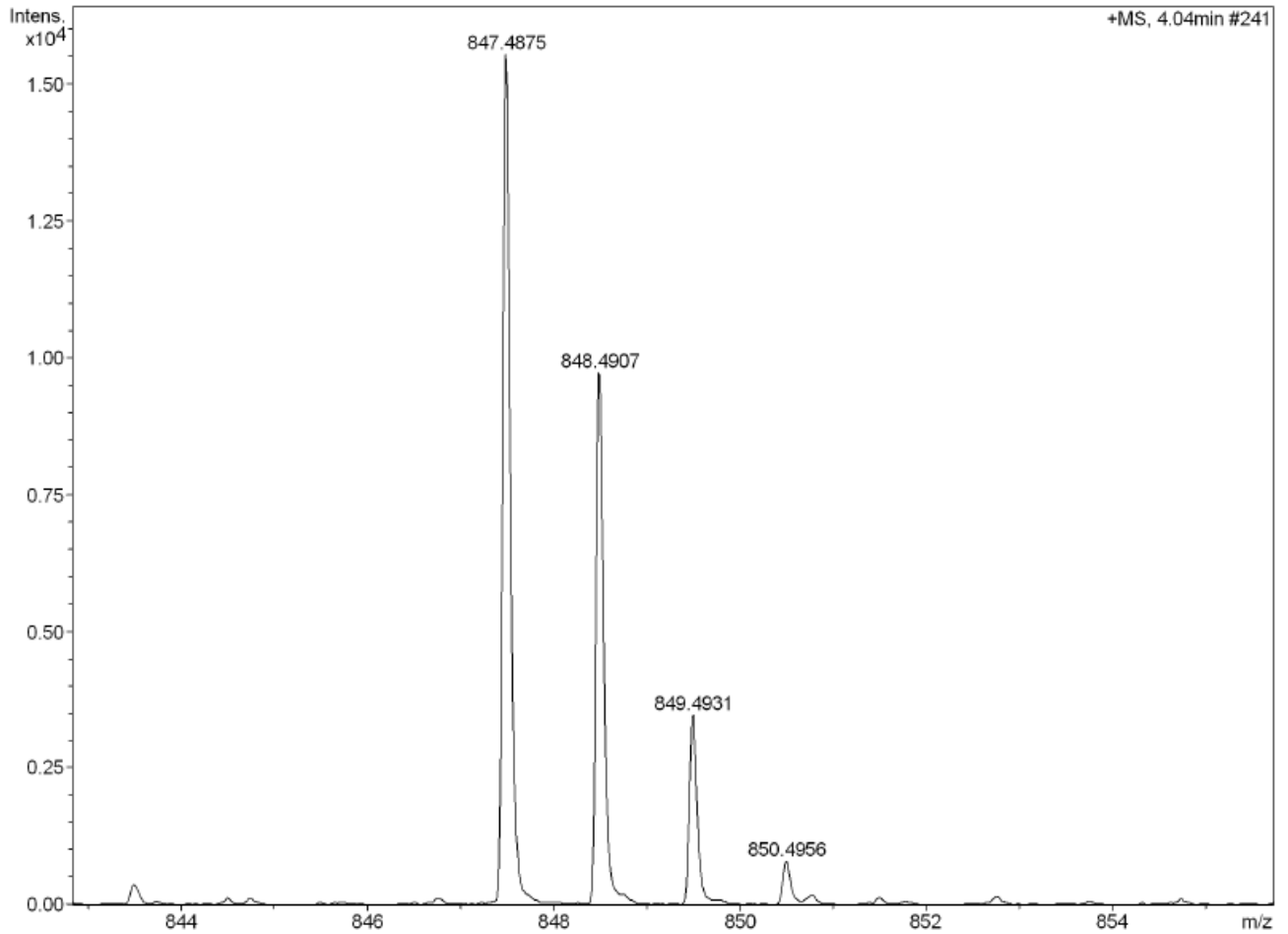

Figure S52. HR mass spectrum (ESI) of 6. 


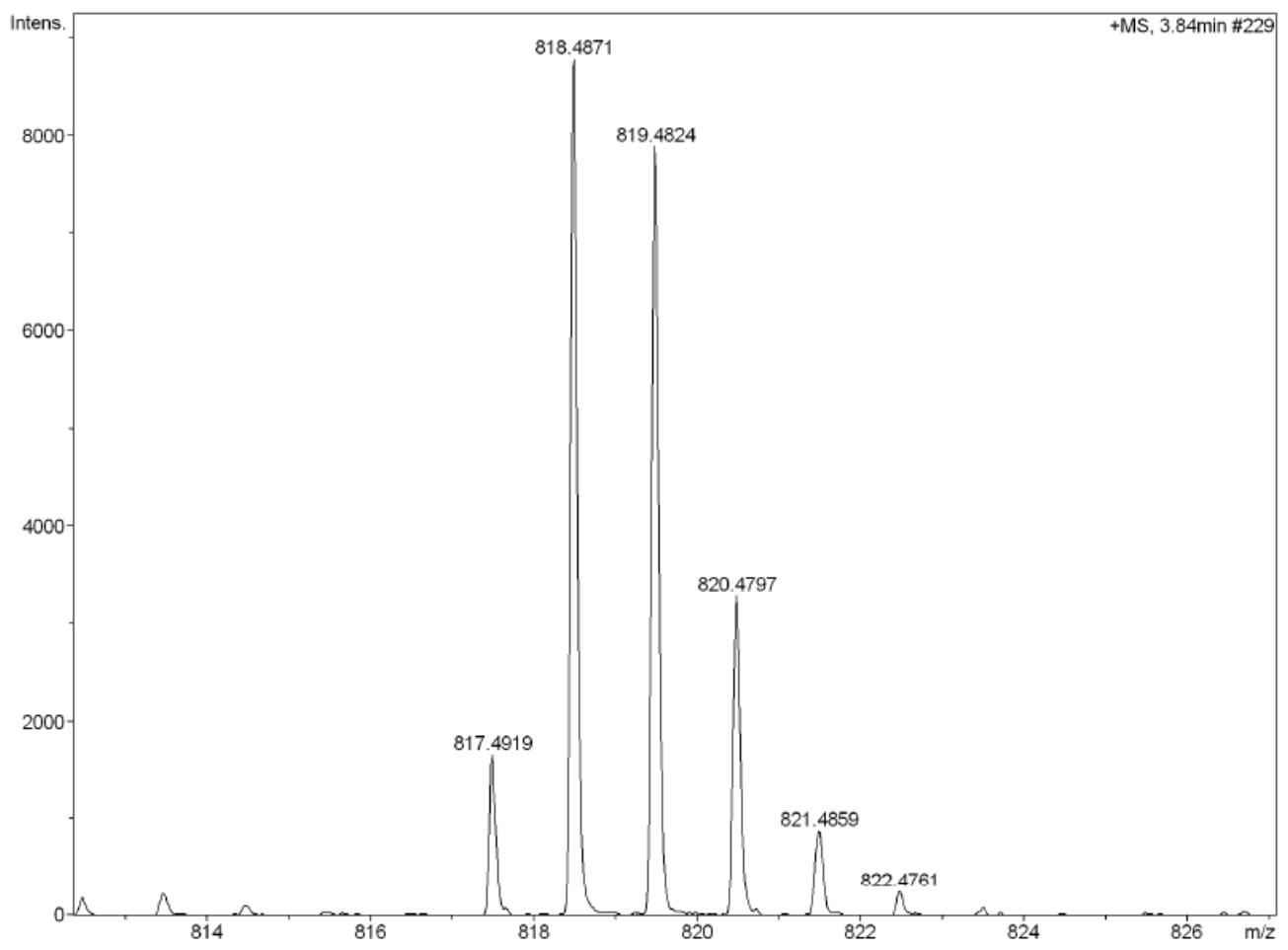

Figure S53. HR mass spectrum (APCI) of 7.

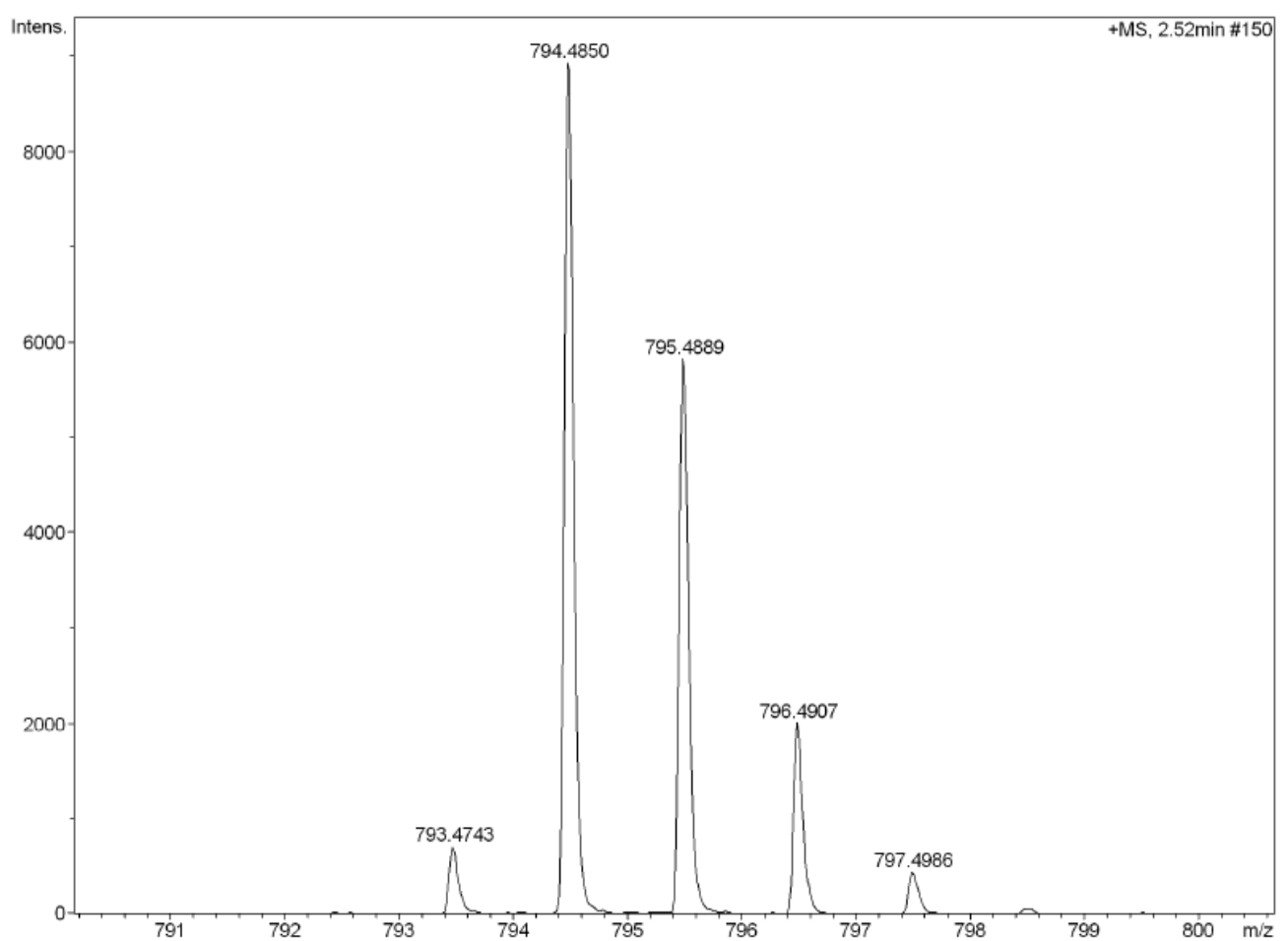

Figure S54. HR mass spectrum (APCI) of radical FR-1. 


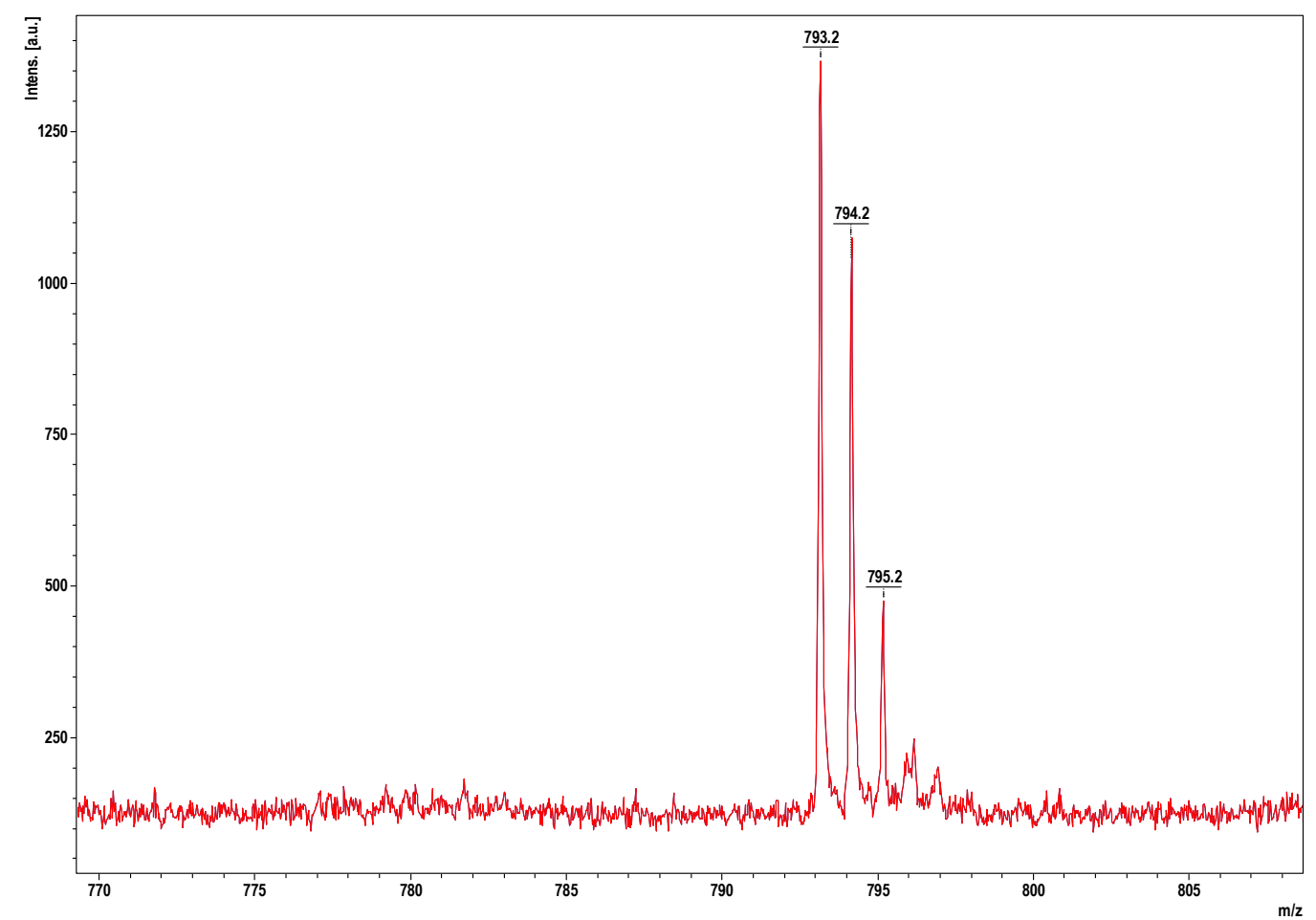

Figure S55. MALDI-TOF mass spectrum of radical FR-1.

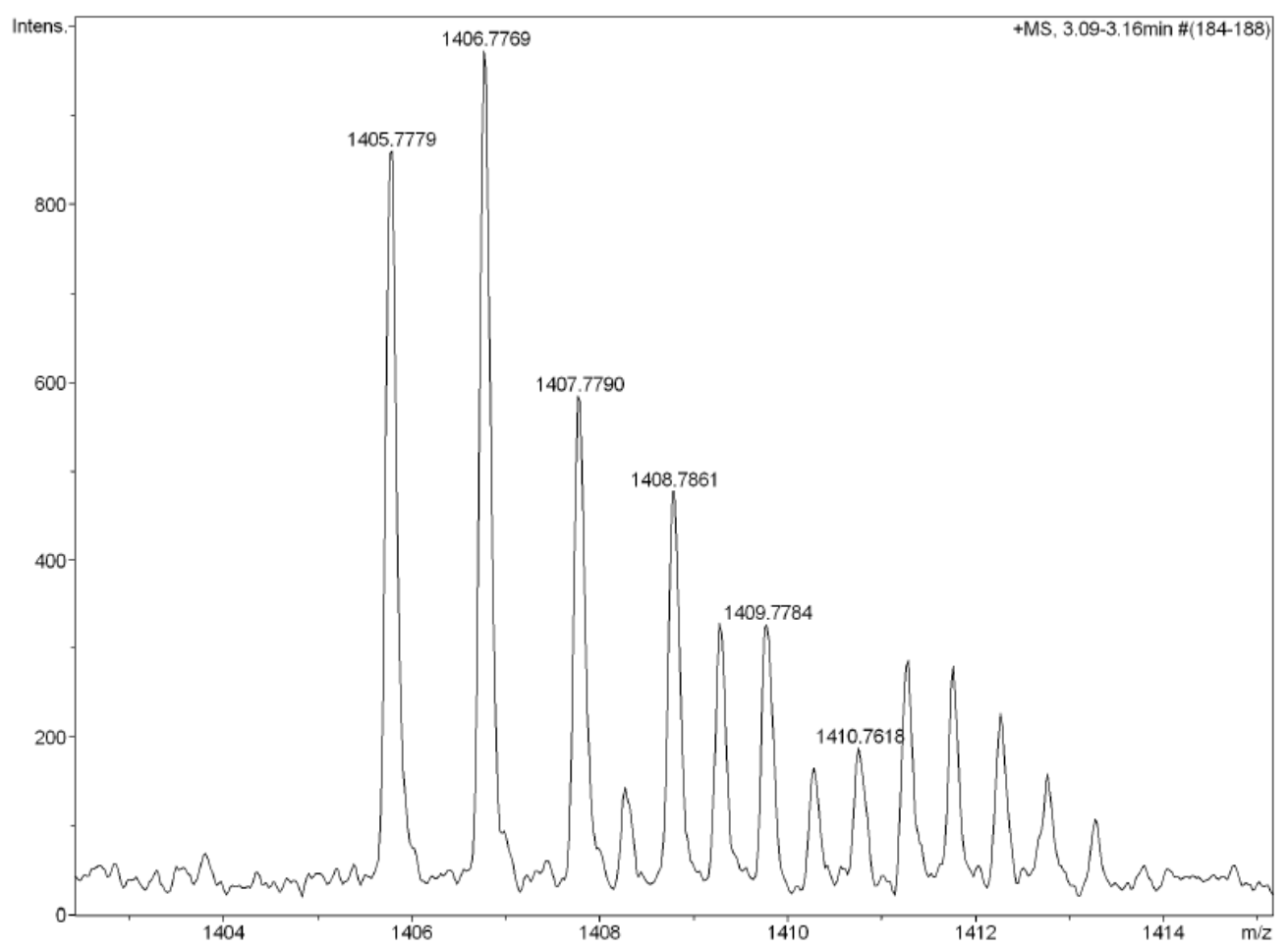

Figure S56. HR mass spectrum (ESI) of 8. 


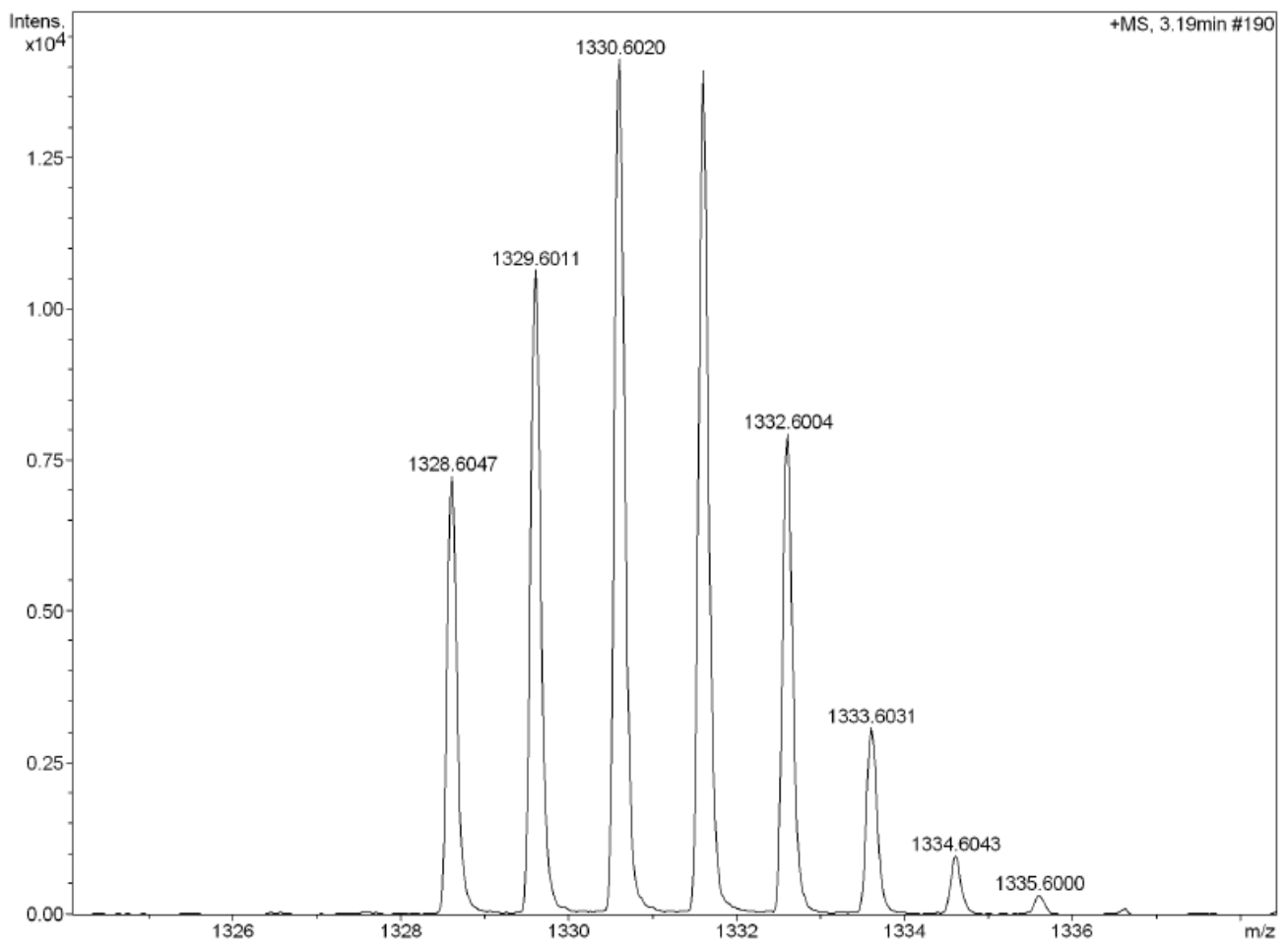

Figure S57. HR mass spectrum (APCI) of compound 9.

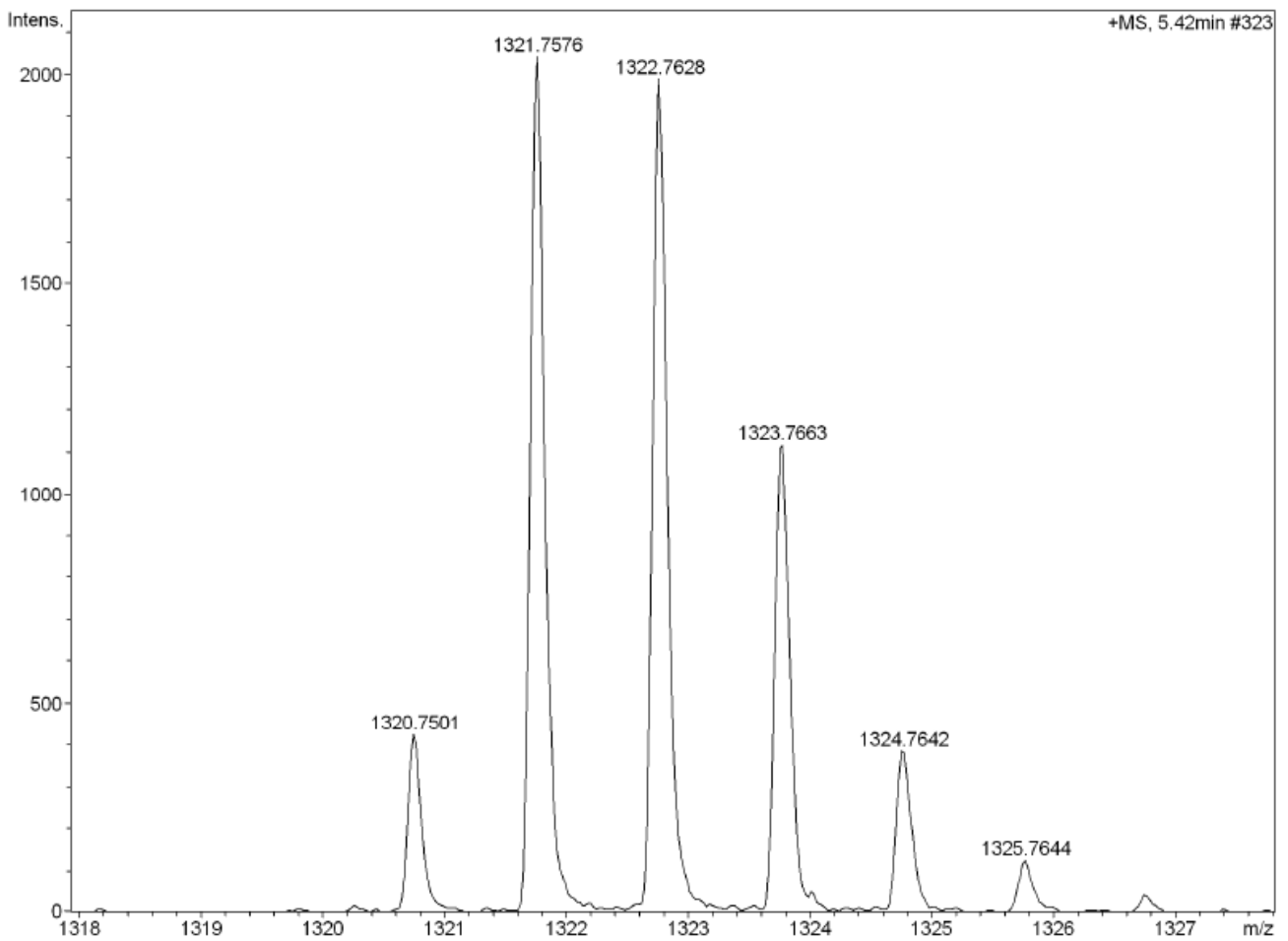

Figure S58. HR mass spectrum (APCI) of radical FR-2. 


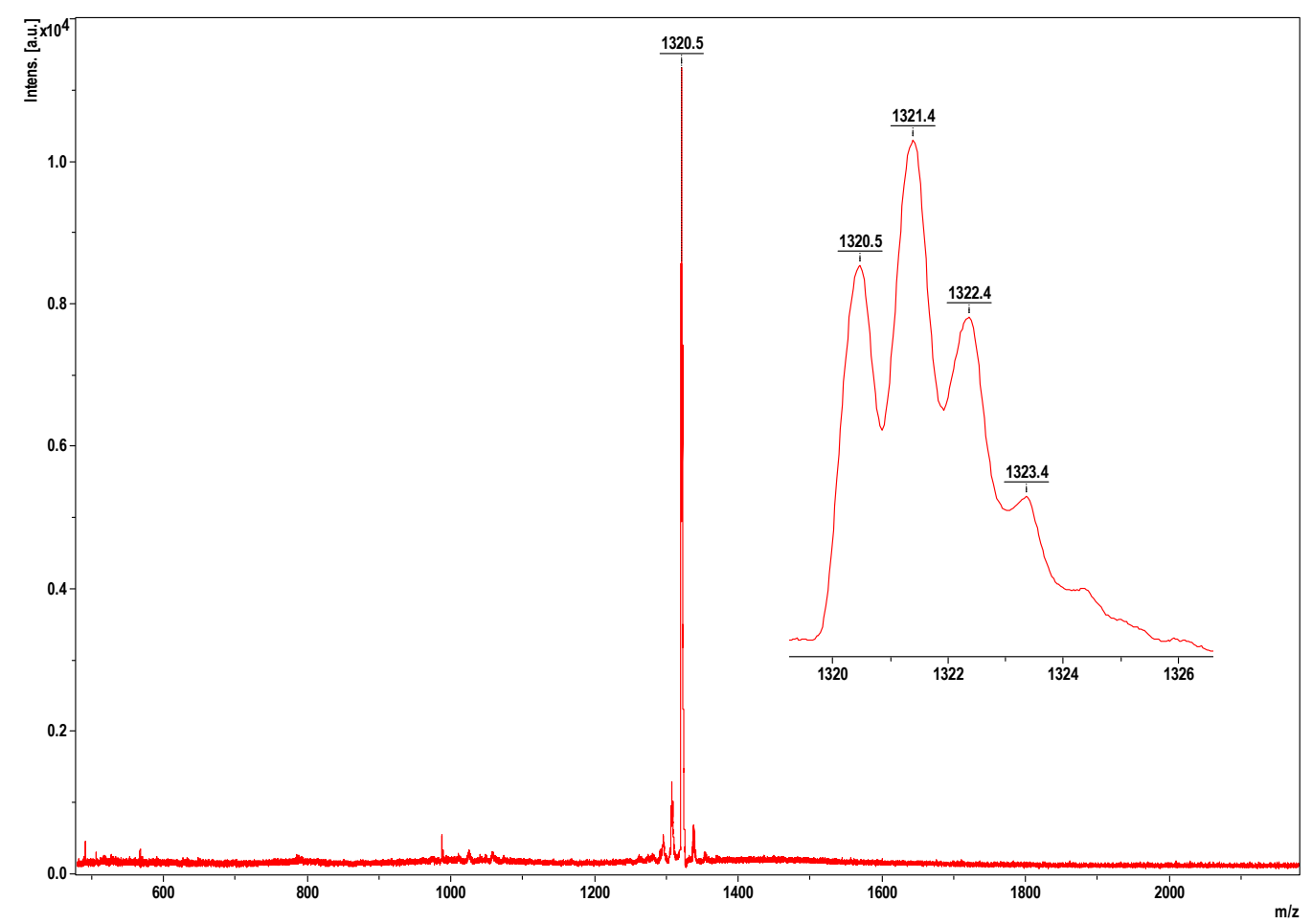

Figure S59. MALDI-TOF mass spectrum of radical FR-2.

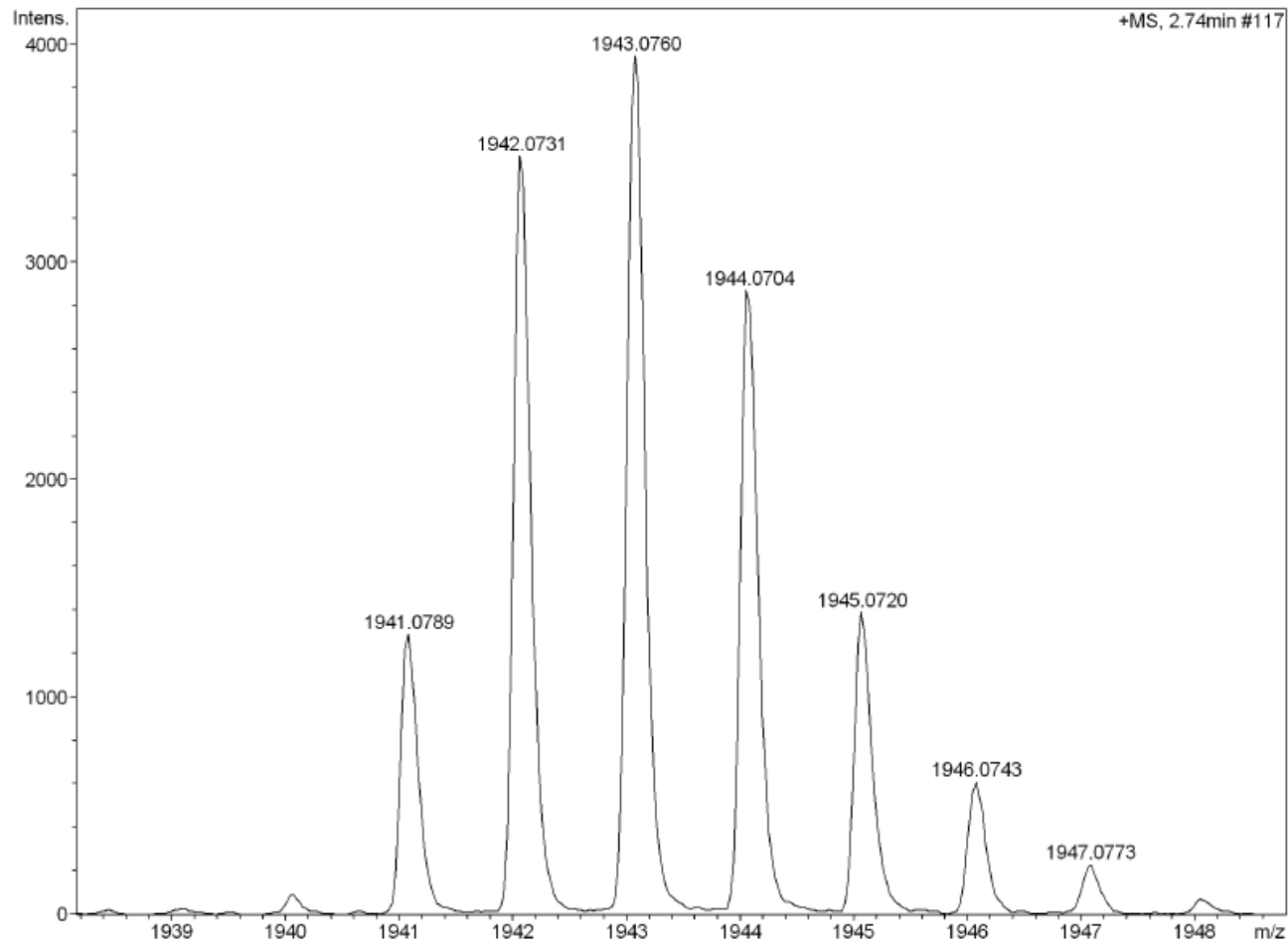

Figure S60. HR mass spectrum (APCI) of $\mathbf{1 0}$. 


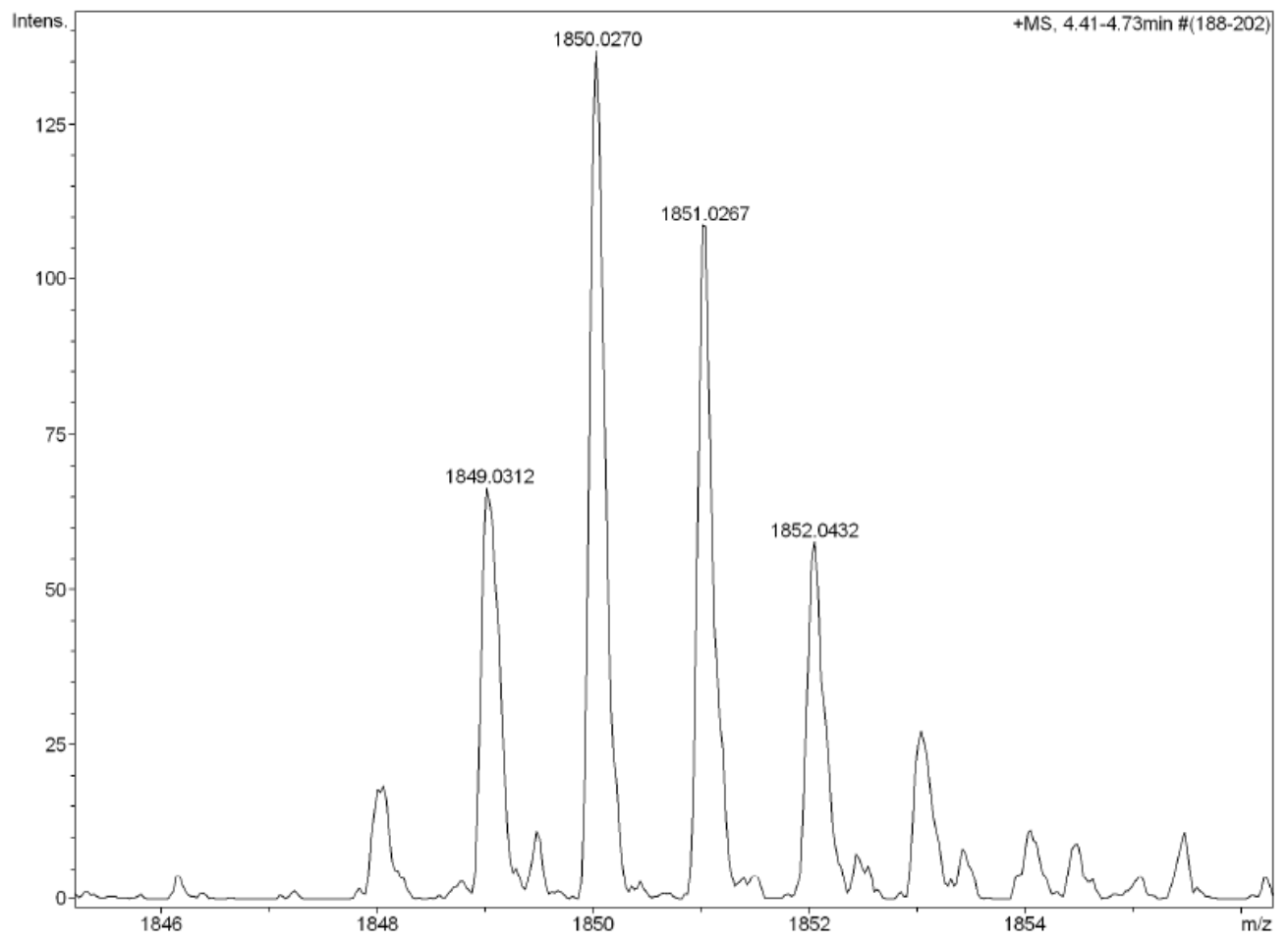

Figure S61. HR mass spectrum (APCI) of radical FR-3.

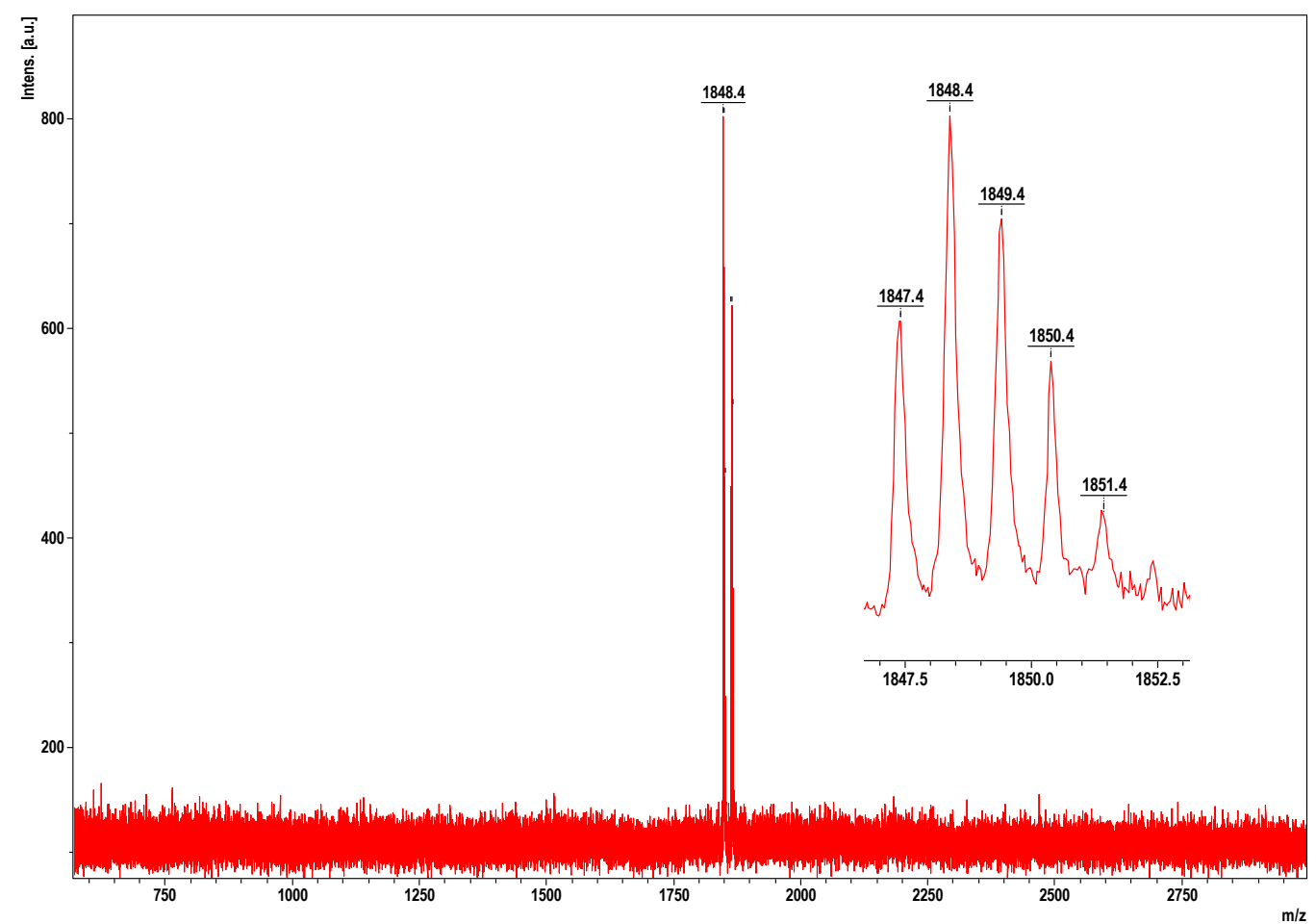

Figure S62. MALDI-TOF mass spectrum of radical FR-3. 


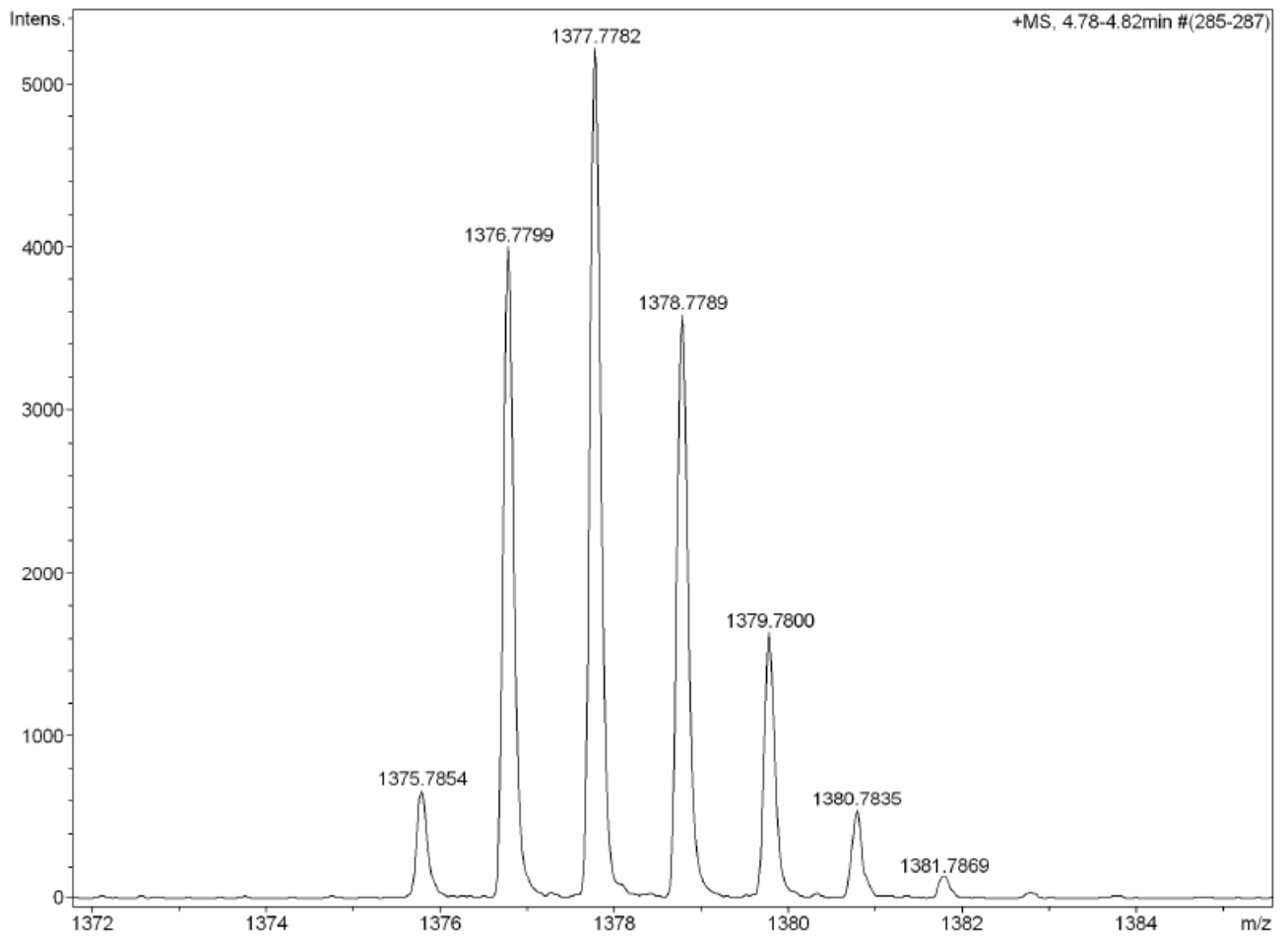

Figure S63. HR mass spectrum (APCI) of compound 11.

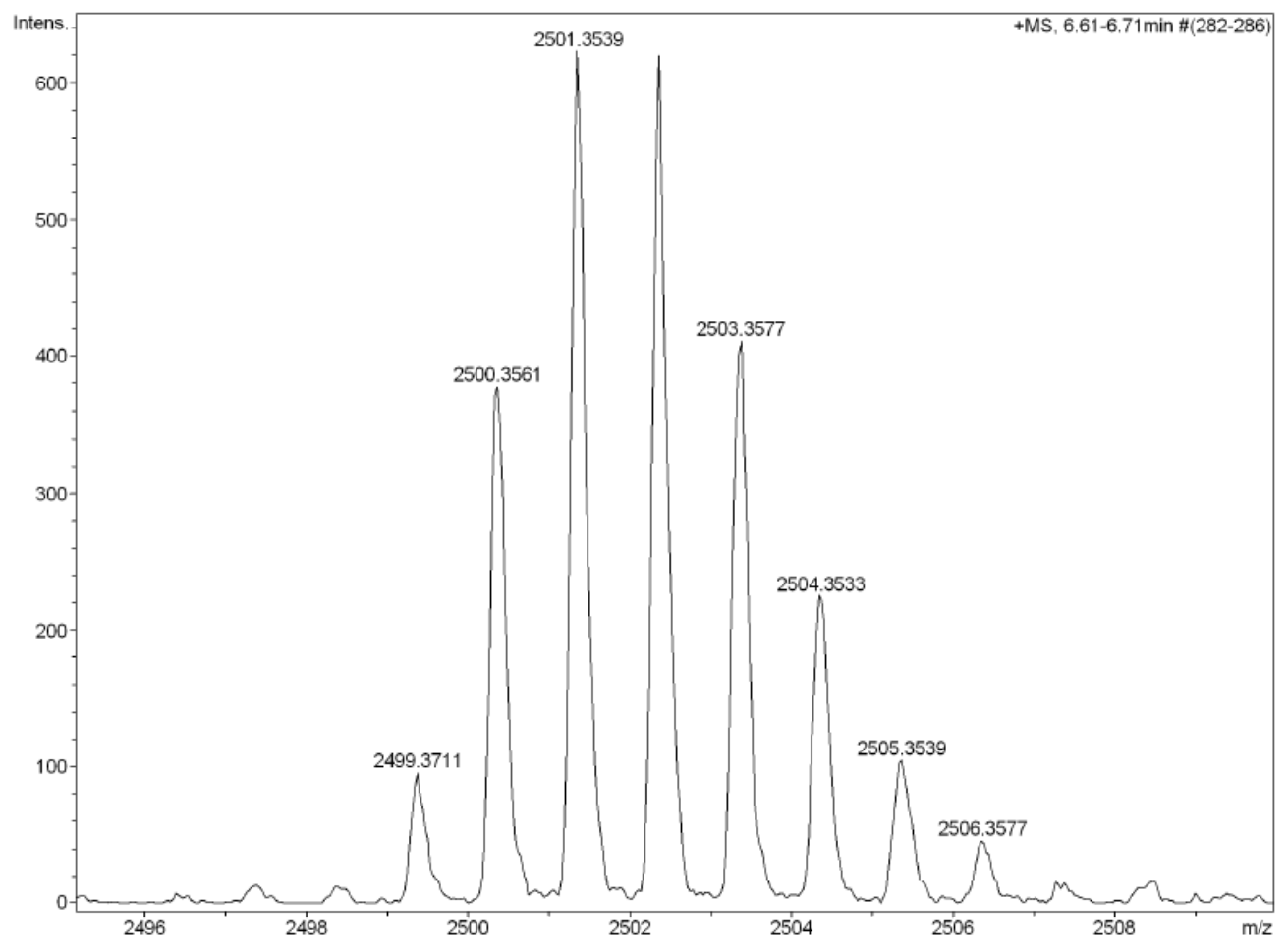

Figure S64. HR mass spectrum (APCI) of compound 12. 


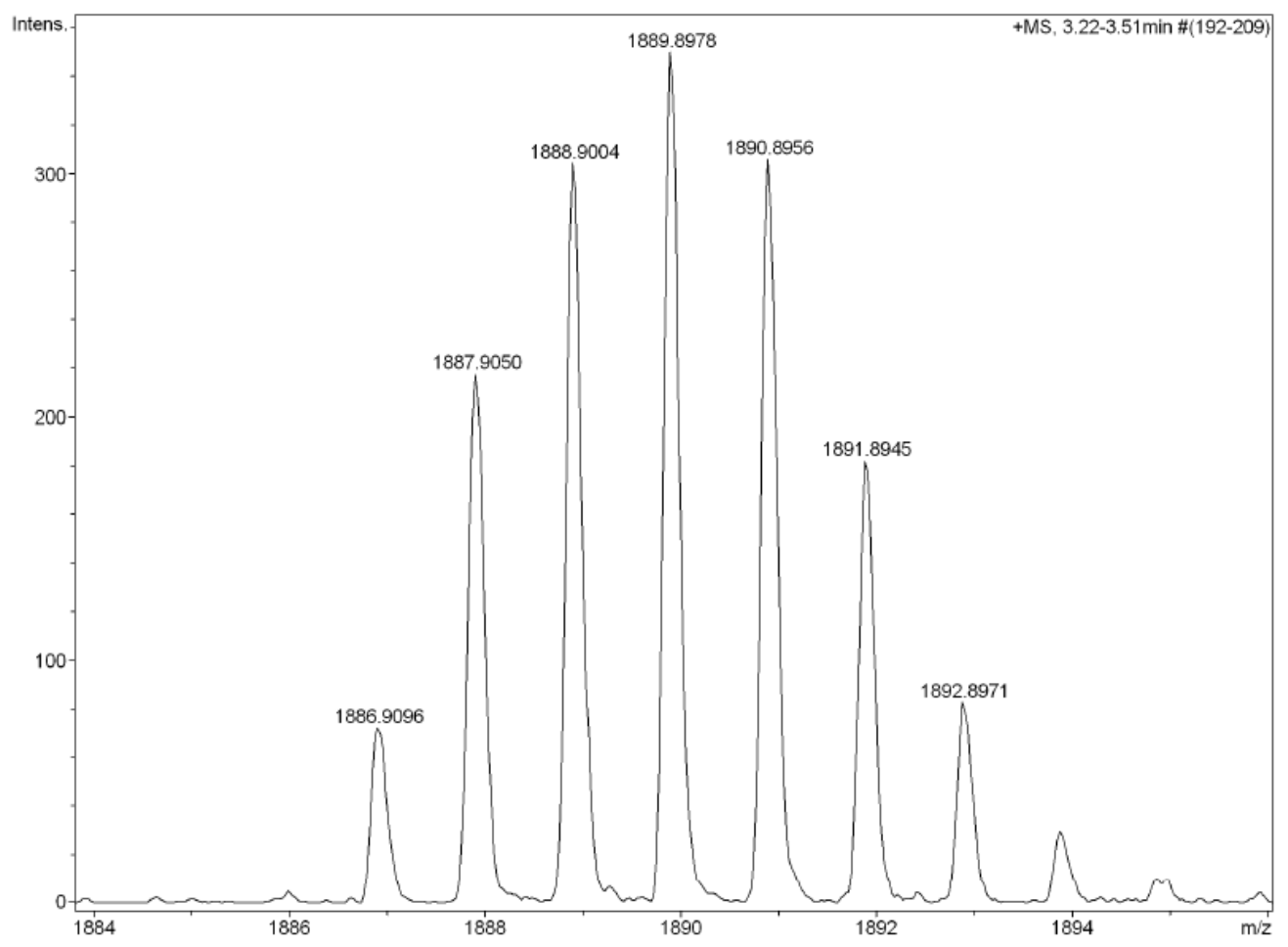

Figure S65. HR mass spectrum (APCI) of 13.

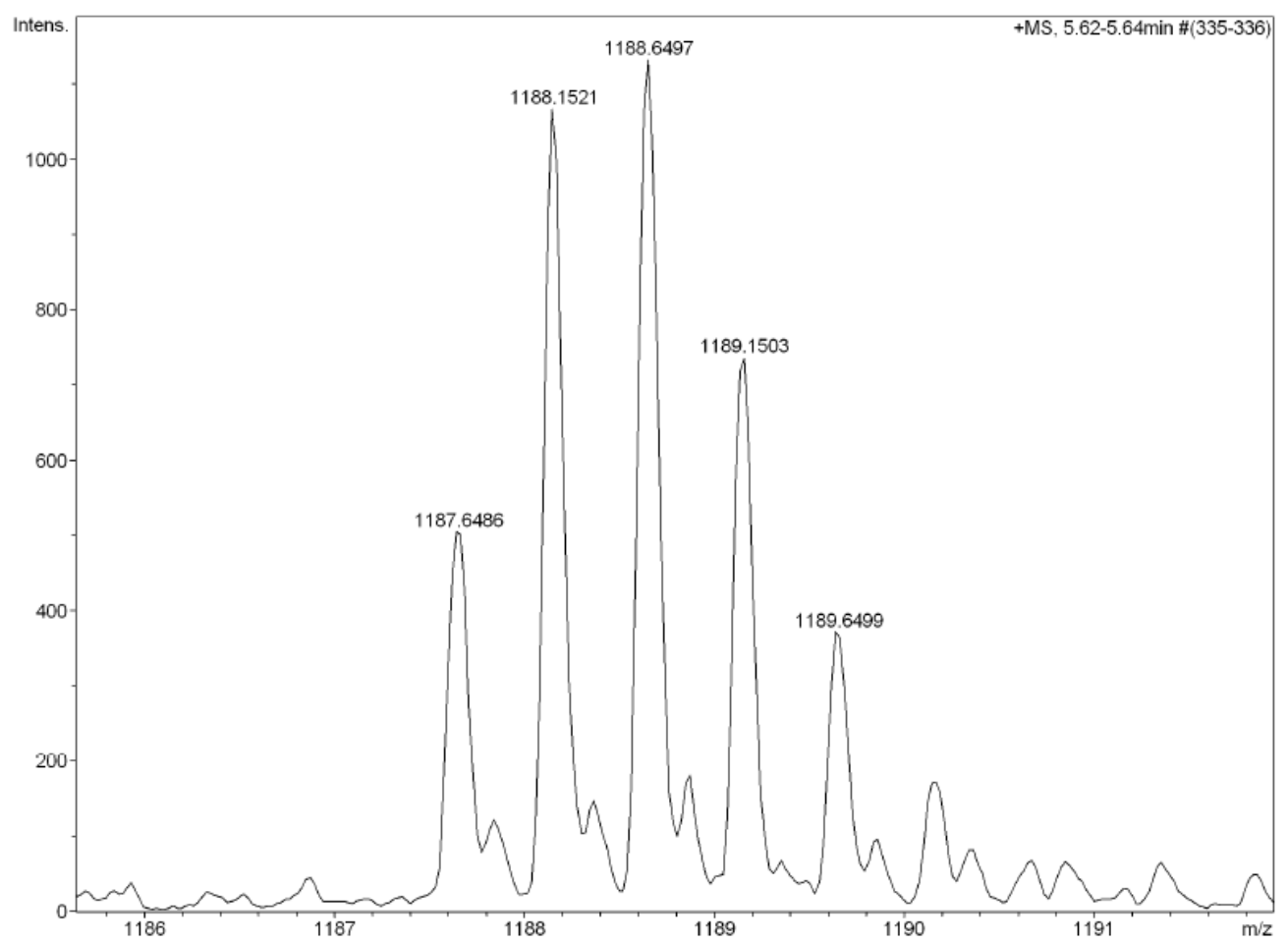

Figure S66. HR mass spectrum (ESI) of radical FR-4. 


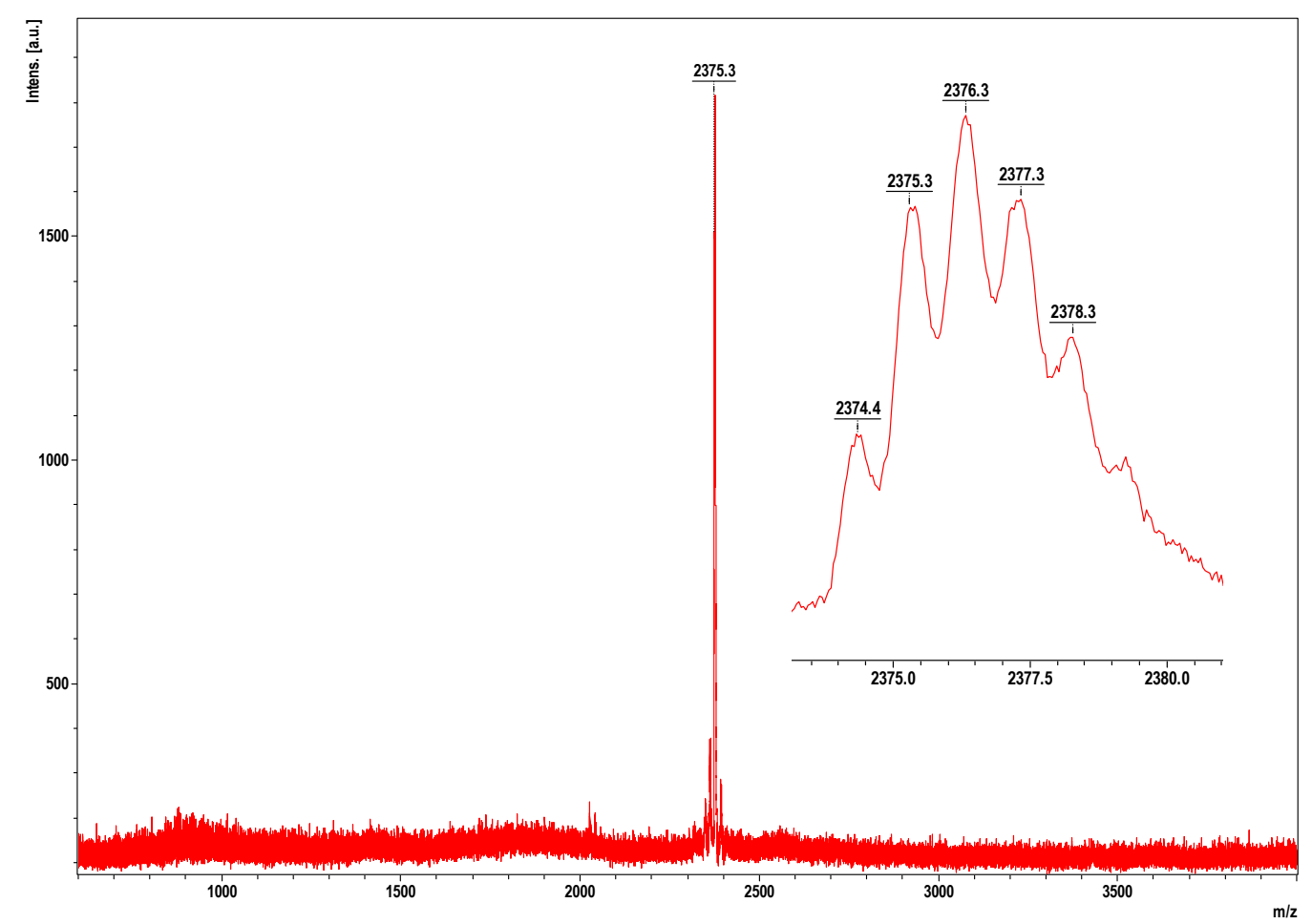

Figure S67. MALDI-TOF mass spectrum of radical FR-4.

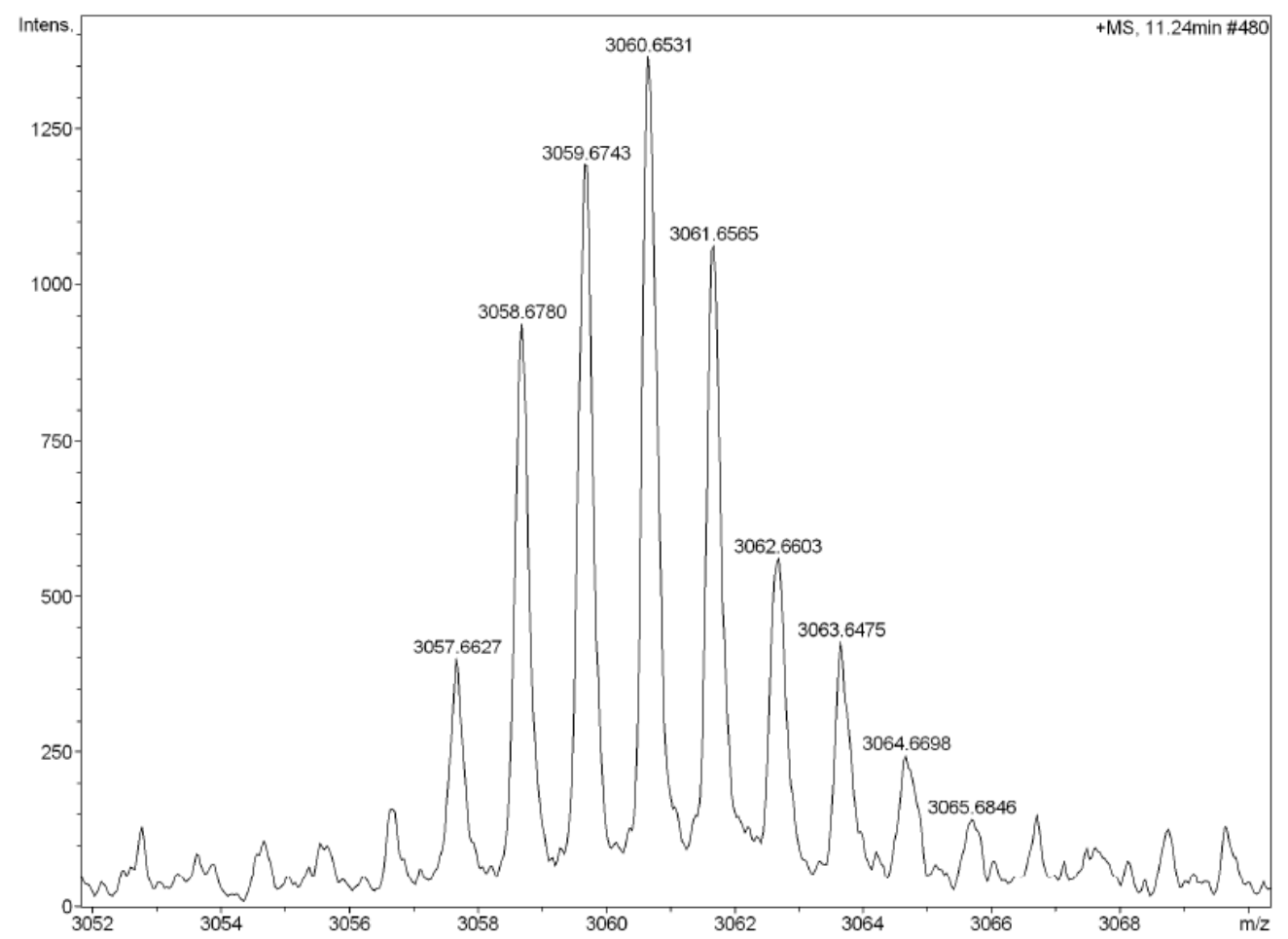

Figure S68. HR mass spectrum (APCI) of 14. 


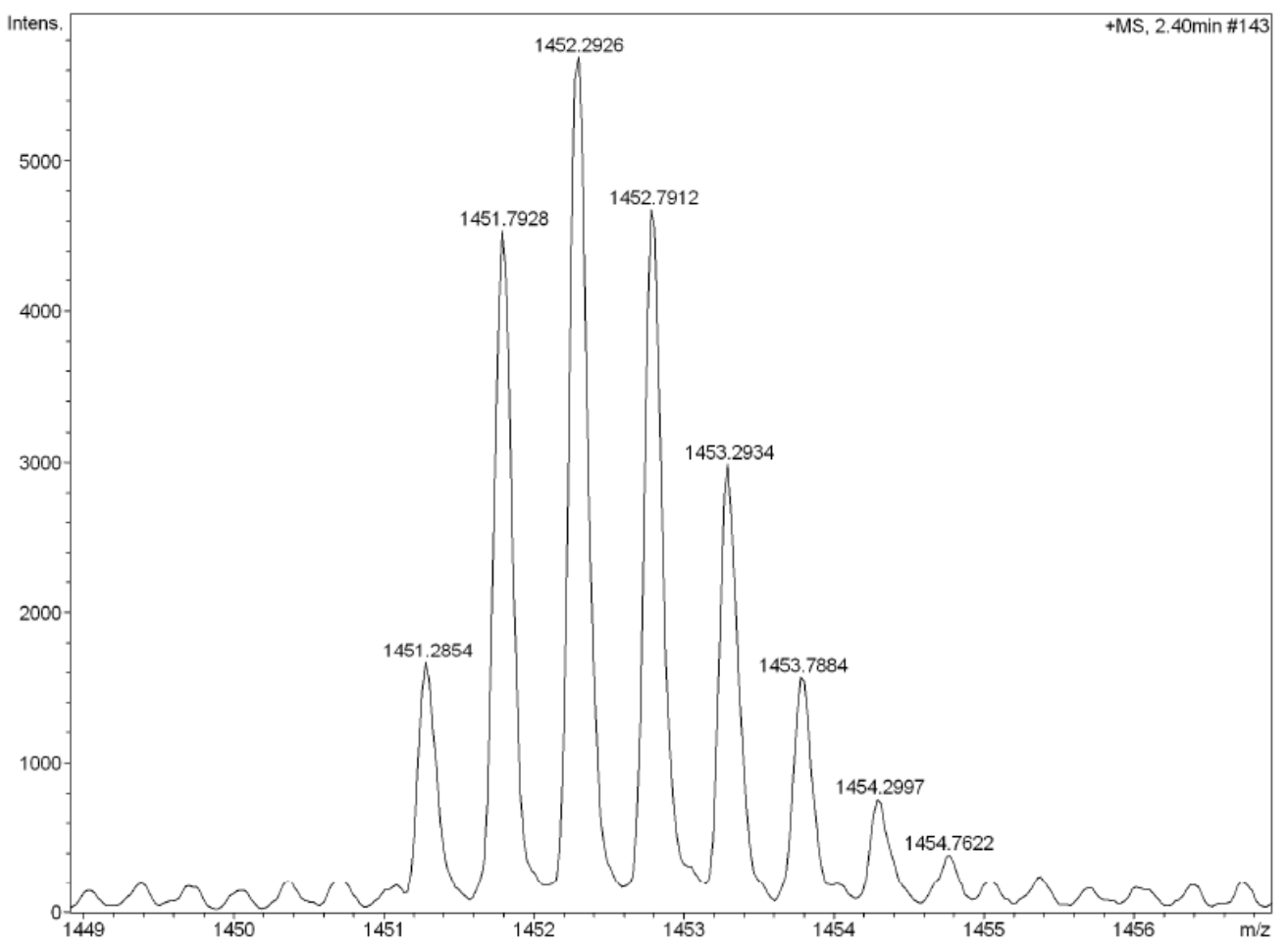

Figure S69. HR mass spectrum (ESI) of radical FR-5.

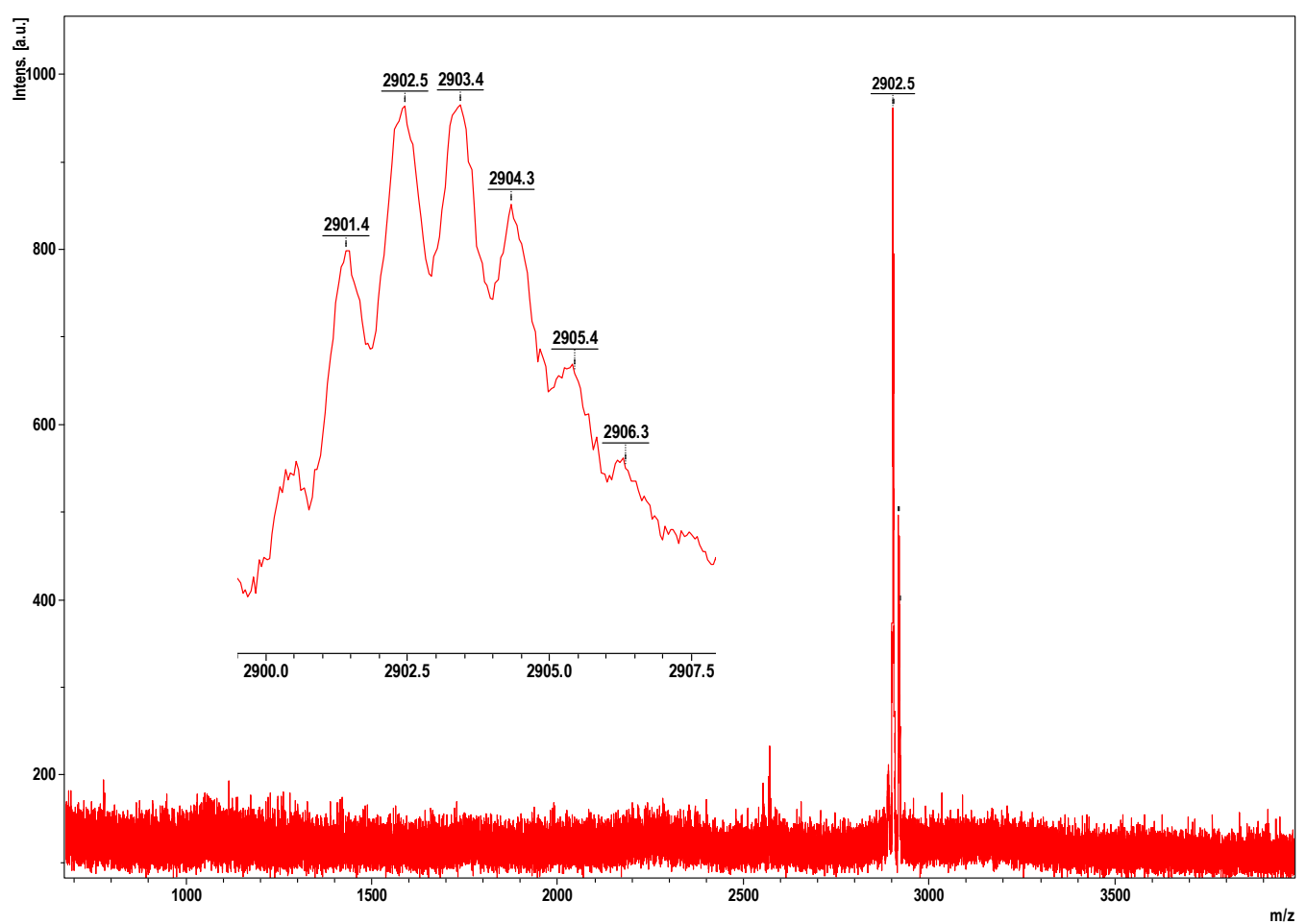

Figure S70. MALDI-TOF mass spectrum of radical FR-5. 


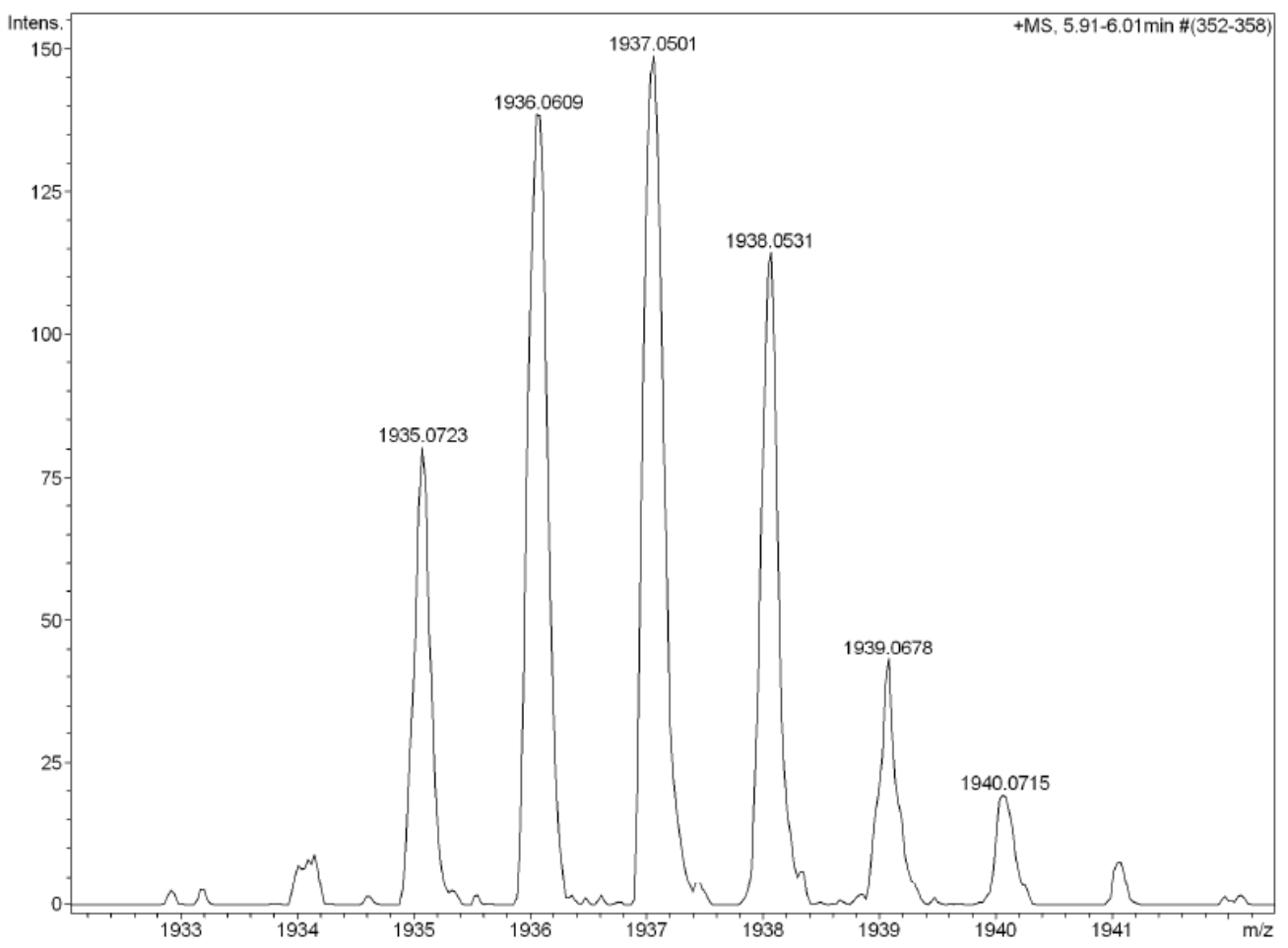

Figure S71. HR mass spectrum (APCI) of $\mathbf{1 5}$.

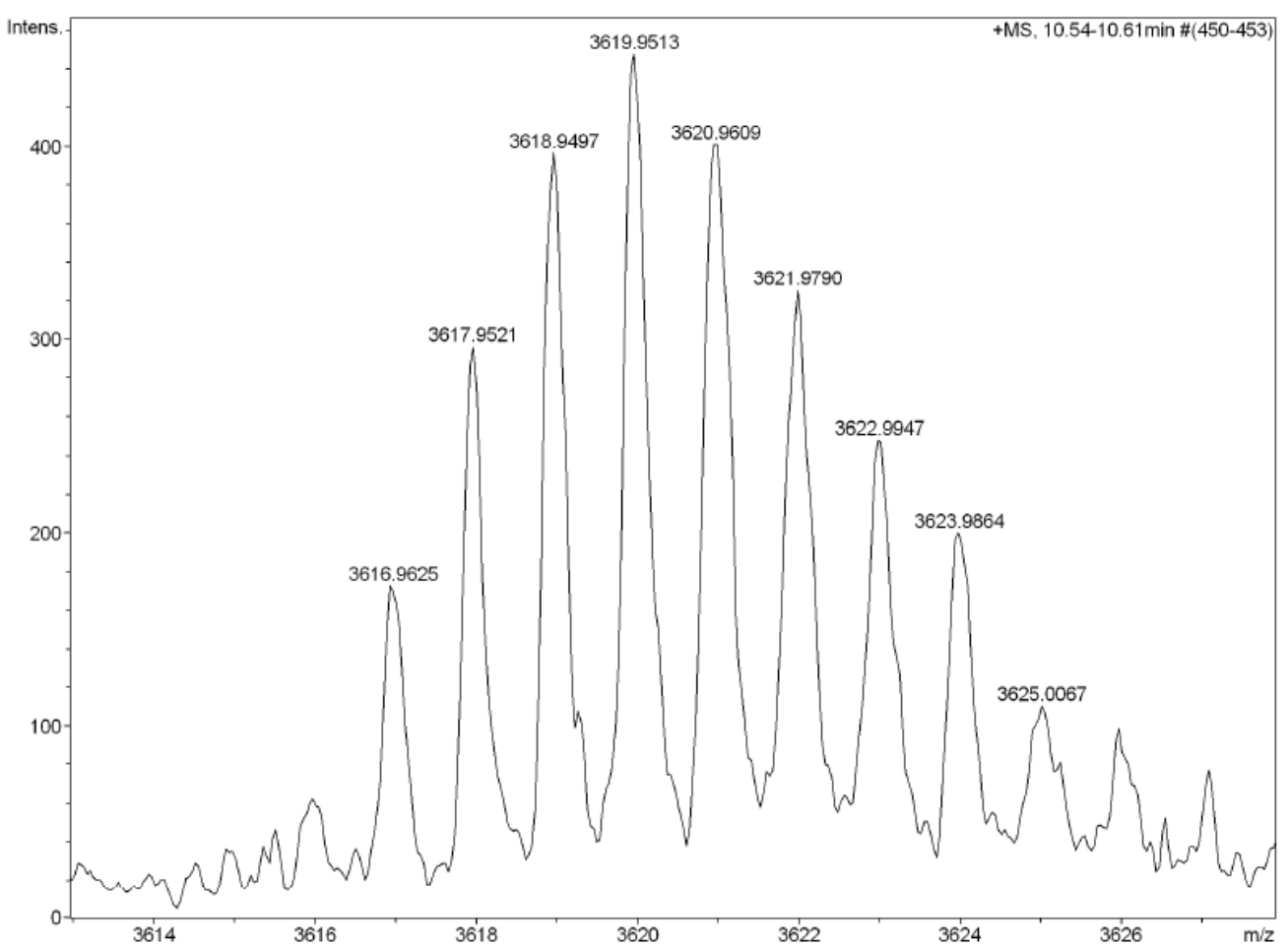

Figure S72. HR mass spectrum (APCI) of compound 16. 


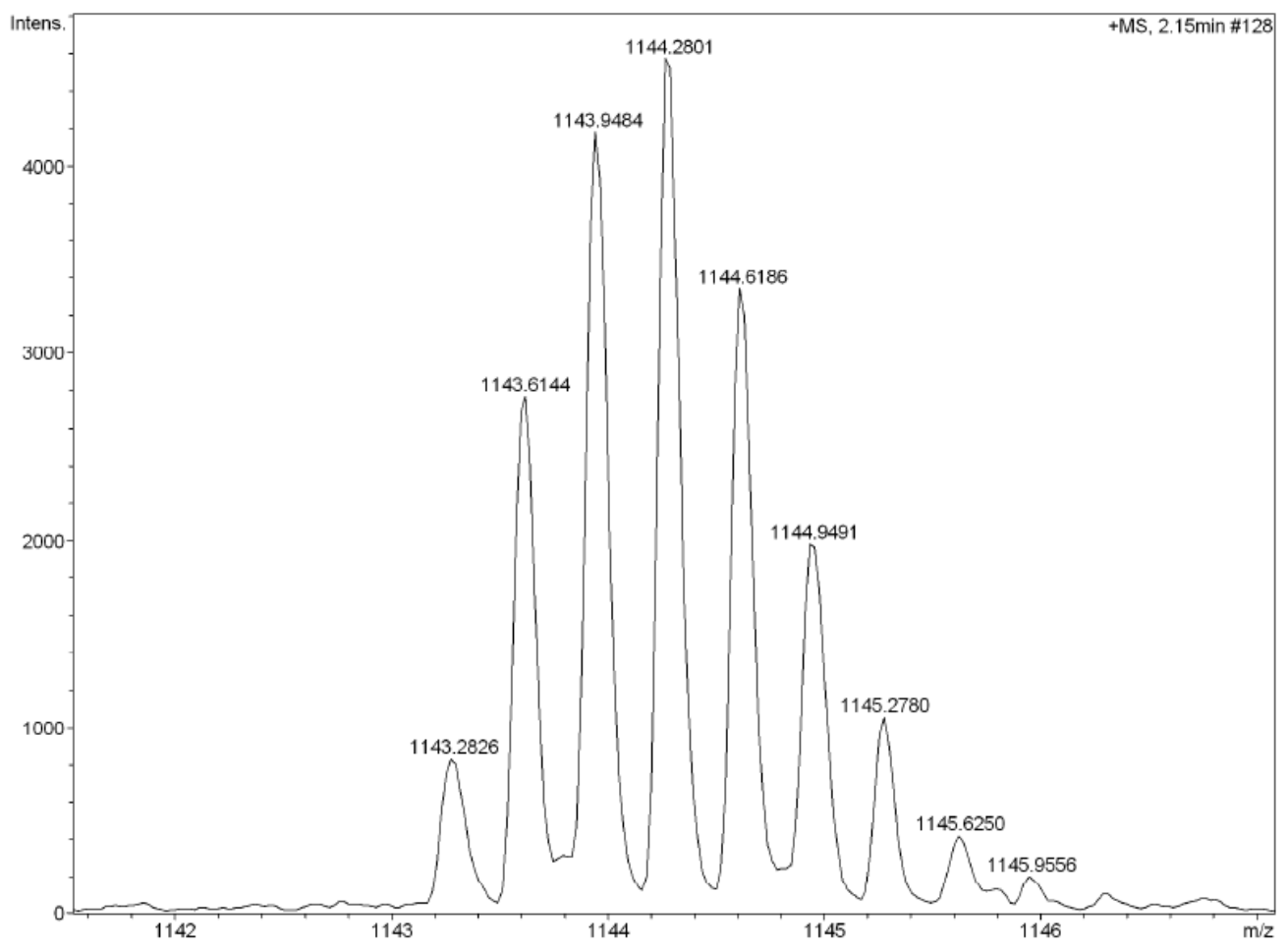

Figure S73. HR mass spectrum (ESI) of radical FR-6.

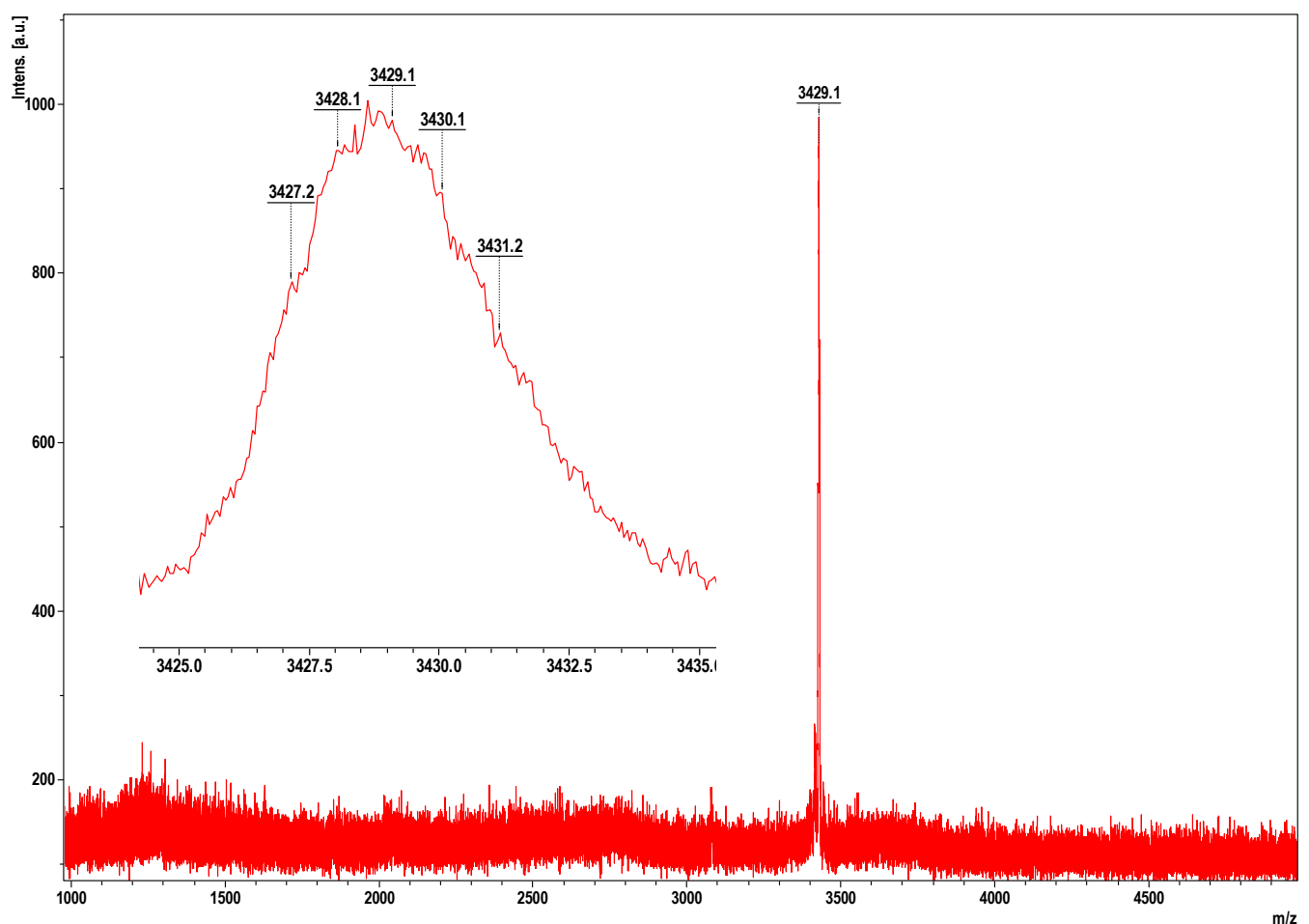

Figure S74. MALDI-TOF mass spectrum of radical FR-6. 


\section{References}

[1] Frisch, M. J.; Trucks, G. W.; Schlegel, H. B.; Scuseria, G. E.; Robb, M. A.; Cheeseman, J. R.; Scalmani, G.; Barone, V.; Mennucci, B.; Petersson, G. A.; Nakatsuji, H.; Caricato, M.; Li, X.; Hratchian, H. P.; Izmaylov, A. F.; Bloino, J.; Zheng, G.; Sonnenberg, J. L.; Hada, M.; Ehara, M.; Toyota, K.; Fukuda, R.; Hasegawa, J.; Ishida, M.; Nakajima, T.; Honda, Y.; Kitao, O.; Nakai, H.; Vreven, T.; Montgomery Jr., J. A.; Peralta, J. E.; Ogliaro, F.; Bearpark, M. J.; Heyd, J.; Brothers, E. N.; Kudin, K. N.; Staroverov, V. N.; Kobayashi, R.; Normand, J.; Raghavachari, K.; Rendell, A. P.; Burant, J. C.; Iyengar, S. S.; Tomasi, J.; Cossi, M.; Rega, N.; Millam, N. J.; Klene, M.; Knox, J. E.; Cross, J. B.; Bakken, V.; Adamo, C.; Jaramillo, J.; Gomperts, R.; Stratmann, R. E.; Yazyev, O.; Austin, A. J.; Cammi, R.; Pomelli, C.; Ochterski, J. W.; Martin, R. L.; Morokuma, K.; Zakrzewski, V. G.; Voth, G. A.; Salvador, P.; Dannenberg, J. J.; Dapprich, S.; Daniels, A. D.; Farkas, Ö.; Foresman, J. B.; Ortiz, J. V.; Cioslowski J.; Fox, D. J. Gaussian 09, Gaussian, Inc., Wallingford, CT, USA, 2009.

[2] (a) Yamaguchi, K.; Fueno, T.; Fukutome, H. Chem. Phys. Lett. 1973, 22, 461. (b) Minami, T.; Nakano, M. J. Phys. Chem. Lett. 2012, 3, 145.

[3] Shao, Y.; Gan, Z.; Epifanovsky, E.; Gilbert, A.T.B.; Wormit, M.; Kussmann, J.; Lange, A.W.; Behn, A.; Deng, J.; Feng, X.; Ghosh, D.; Goldey, M.; Horn, P.R.; Jacobson, L.D.; Kaliman, I.; Khaliullin, R.Z.; Kus, T.; Landau, A.; Liu, J.; Proynov, E.I.; Rhee, Y.M.; Richard, R.M.; Rohrdanz, M.A.; Steele, R.P.; Sundstrom, E.J.; Woodcock III, H.L.; Zimmerman, P.M.; Zuev, D.; Albrecht, B.; Alguires, E.; Austin, B.; Beran, G.J.O.; Bernard, Y.A.; Berquist, E.; Brandhorst, K.; Bravaya, K.B.; Brown, S.T.; Casanova, D.; Chang, C.-M.; Chen, Y.; Chien, S.H.; Closser, K.D.; Crittenden, D.L.; Diedenhofen, M.; DiStasio Jr., R.A.; Do, H.; Dutoi, A.D.; Edgar, R.G.; Fatehi, S.; Fusti-Molnar, L.; Ghysels, A.; GolubevaZadorozhnaya, A.; Gomes, J.; Hanson-Heine, M.W.D.; Harbach, P.H.P.; Hauser, A.W.; Hohenstein, E.G.; Holden, Z.C.; Jagau, T.-C.; Ji, H.; Kaduk, B.; Khistyaev, K.; Kim, J.; Kim, J.; King, R.A.; Klunzinger, P.; Kosenkov, D.; Kowalczyk, T.; Krauter, C.M.; Laog, K.U.; Laurent, A.; Lawler, K.V.; Levchenko, S.V.; Lin, C.Y.; Liu, F.; Livshits, E.; Lochan, R.C.; Luenser, A.; Manohar, P.; Manzer, S.F.; Mao, S.-P.; Mardirossian, N.; Marenich, A.V.; Maurer, S.A.; Mayhall, N.J.; Oana, C.M.; Olivares-Amaya, R.; O’Neill, D.P.; Parkhill, J.A.; Perrine, T.M.; Peverati, R.; Pieniazek, P.A.; Prociuk, A.; Rehn, D.R.; Rosta, E.; Russ, N.J.; Sergueev, N.; Sharada, S.M.; Sharmaa, S.; Small, D.W.; Sodt, A.; Stein, T.; Stuck, D.; Su, Y.-C.; Thom, A.J.W.; Tsuchimochi, T.; Vogt, L.; Vydrov, O.; Wang, T.; Watson, M.A.; Wenzel, J.; White, A.; Williams, C.F.; Vanovschi, V.; Yeganeh, S.; Yost, S.R.; You, Z.-Q.; Zhang, I.Y.; Zhang, X.; Zhou, Y.; Brooks, B.R.; Chan, G.K.L.; Chipman, D.M.; Cramer, C.J.; Goddard III, W.A.; Gordon, M.S.; Hehre, W.J.; Klamt, A.; Schaefer III, H.F.; Schmidt, M.W.; Sherrill, C.D.; Truhlar, D.G.; Warshel, A.; Xu, X.; Aspuru-Guzik, A.; Baer, R.; Bell, A.T.; Besley, N.A.; Chai, J.-D.; Dreuw, A.; Dunietz, B.D.; 
Furlani, T.R.; Gwaltney, S.R.; Hsu, C.-P.; Jung, Y.; Kong, J.; Lambrecht, D.S.; Liang, W.Z.; Ochsenfeld, C.; Rassolov, V.A.; Slipchenko, L.V.; Subotnik, J.E.; Van Voorhis, T.; Herbert, J.M.; Krylov, A.I.; Gill, P.M.W.; Head-Gordon, M. Mol. Phys., 2015, 113, 184.

[4] (a) Casanova, D.; Head-Gordon, M. Phys. Chem. Chem. Phys. 2009, 11, 9779. (b) HeadGordon, M. Chem. Phys. Lett. 2003, 372, 508. 